\title{
The sumoylation and neddylation networks in Aspergillus nidulans development
}

\author{
Dissertation \\ zur Erlangung des mathematisch-naturwissenschaftlichen Doktorgrades \\ "Doctor rerum naturalium" \\ der Georg-August-Universität Göttingen \\ im Promotionsprogramm Biologie \\ der Georg-August University School of Science (GAUSS)
}

vorgelegt von

Rebekka Harting

aus Göttingen

Göttingen 2013 


\section{Betreuungsausschuss:}

Prof. Dr. Gerhard H. Braus, Institut für Mikrobiologie und Genetik, Georg-August Universität Göttingen.

Prof. Dr. Andrea Polle, Abteilung Forstbotanik u. Baumphysiologie, Georg-August Universität Göttingen.

\section{Mitglieder der Prüfungskommission:}

Referent:

Prof. Dr. Gerhard H. Braus, Institut für Mikrobiologie und Genetik, Georg-August Universität Göttingen.

Korreferent:

Prof. Dr. Andrea Polle, Abteilung Forstbotanik u. Baumphysiologie, Georg-August Universität Göttingen.

Weitere Mitglieder der Prüfungskommission:

Prof. Dr. Stefanie Pöggeler, Institut für Mikrobiologie und Genetik, Georg-August Universität Göttingen.

Jun.-Prof. Dr. Kai Heimel, Institut für Mikrobiologie und Genetik, Georg-August Universität Göttingen.

PD Dr. Stefan Irninger, Institut für Mikrobiologie und Genetik, Georg-August Universität Göttingen.

PD Dr. Michael Hoppert, Institut für Mikrobiologie und Genetik, Georg-August Universität Göttingen.

Tag der mündlichen Prüfung: 19.06.2013 

This work was accomplished in the group of Prof. Dr. Gerhard H. Braus, at the Department of Molecular Microbiology and Genetics at the Institute of Microbiology and Genetics, Georg-August University Göttingen.

Parts of this work are published/accepted for publication:

M. R. von Zeska Kress, R. Harting, O. Bayram, M. Christmann, H. Irmer, O. Valerius, J. Schinke, G. H. Goldman \& G. H. Braus (2012) The COP9 signalosome counteracts the accumulation of cullin SCF ubiquitin E3 RING ligases during fungal development. Mol Microbiol 83: 1162-1177.

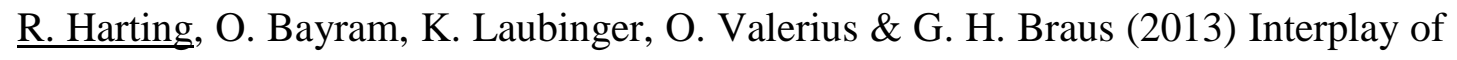
the fungal sumoylation network for control of multicellular development. Mol Microbiol, accepted for publication 30.09.2013. 


\section{Table of contents}

$\begin{array}{ll}\text { Summary } & 1\end{array}$

$\begin{array}{ll}\text { Zusammenfassung } & 2\end{array}$

1. Introduction 4

1.1. Protein modification by members of the ubiquitin-like protein family 4

1.1.1. Post-translational modification of proteins 4

1.1.2. The family of ubiquitin-like proteins 4

1.2. Nedd8 and the control of ubiquitin dependent protein degradation 6

1.2.1. Ubiquitin dependent protein degradation via the 26S proteasome 6

1.2.2. Nedd8 and the control of cullin RING E3-ubiquitin ligases 7

1.2.2.1 Neddylation of cullin RING ligases 7

1.2.2.2. Deneddylation of cullins by the COP9 signalosome 10

1.2.2.3 Modification of cullin by Cand1 11

1.2.2.4. The deneddylase Den1 12

1.3. The small ubiquitin-like modifier Sumo 12

$\begin{array}{lll}\text { 1.3.1. Consequences of sumoylation } & 13\end{array}$

$\begin{array}{lll}\text { 1.3.2. The Sumo attachment pathway } & 15\end{array}$

$\begin{array}{ll}\text { 1.3.3. The Sumoylation motif } & 17\end{array}$

$\begin{array}{ll}\text { 1.3.4. Desumoylation } & 18\end{array}$

1.4. The model organism Aspergillus nidulans 19

$\begin{array}{ll}\text { 1.4.1. Vegetative growth } & 20\end{array}$

1.4.2. Sexual reproduction 20

1.4.3. Asexual reproduction 22

1.4.4. Regulation of development 22

1.4.5. Neddylation and deneddylation in A. nidulans 25

1.4.6. The SumO protein of Aspergillus nidulans 26

$\begin{array}{ll}\text { 1.5. Scope and aim of this study } & 28\end{array}$

2. Materials and Methods $\quad 29$

2.1. Materials 29

2.1.1. Growth media and growth conditions 29

2.1.1.1. Escherichia coli 29

2.1.1.2. Aspergillus nidulans 29 
2.1.2. Strains, plasmids and primers 30

2.1.2.1. E. coli strains 30

2.1.2.2. Plasmids used in this study 30

2.1.2.3. Primers used in this study 32

2.1.2.4. Aspergillus nidulans strains used in this study 37

2.1.2.5. Plasmid and strain constructions 39

2.1.2.5.1. Plasmid and strain construction for the TAP::SumO strains 39

2.1.2.5.2. PCR-mediated construction of deletion cassettes and deletion strain construction for $u l p A, u l p B, \operatorname{aos} A, u b a B, u b c N$ and sizA 40

2.1.2.5.3. Plasmid and strain construction for complementation of the ulpA deletion strain

2.1.2.5.4. Plasmid and strain construction for complementation of the $u l p B$ deletion strain

2.1.2.5.5. Plasmid and strain construction for complementation of the aosA deletion strain

2.1.2.5.6. Plasmid and strain construction for complementation of the $u b a B$ deletion strain

2.1.2.5.7. Plasmid and strain construction for complementation of the $u b c N$ deletion strain

2.1.2.5.8. Plasmid and strain construction for $\operatorname{den} A$ overexpression 43

2.1.2.5.9. Plasmid and strain construction for setA deletion 43

2.1.2.5.10. Complementation of the setA deletion strain 43

2.1.2.5.11. Plasmid and strain construction for SetA::TAP 44

2.1.2.5.12. Plasmid and strain construction for expression of histone::mrfp and setA overexpression

2.1.2.5.13. Construction of the $d c n A, A N 4149$ and AN4491 deletion strains 45

2.1.2.5.14. Construction of the $r b x A$ deletion cassette and heterokaryon rescue $\quad 46$

2.1.2.5.15. Plasmid and strain construction for DenA BiFC experiments 46

2.1.2.5.16. Strain construction for fbox $15:: g f p$ overexpression 47

$\begin{array}{lll}\text { 2.2. Methods } & 48\end{array}$

2.2.1 Genetic manipulations and molecular methods 48

2.2.1.1. Transformation procedures 48

$\begin{array}{lll}\text { 2.2.1.2. } & \text { Sequence analyses } & 48\end{array}$

2.2.1.3. Recombinant DNA methods 49

2.2.1.4. DNA isolation and hybridization $\quad 49$

2.2.1.5. RNA isolation and cDNA synthesis $\quad 49$

2.2.1.6. Quantitative real-time PCR 50

$\begin{array}{lll}\text { 2.2.2. Protein methods } & 50\end{array}$ 
2.2.2.1. Protein isolation and analyses $\quad 50$

2.2.2.2. Antibodies 51

2.2.2.3. Tandem Affinity Purification (TAP) 51

2.2.2.4. Coomassie staining 52

2.2.2.5. Tryptic in-gel digestion of protein samples 52

2.2.2.6. Protein identification by mass spectrometry 53

2.2.2.6.1. NanoLC-NanoESI mass spectrometry analyses and database search (TAP::SumO) 53

2.2.2.6.2. Mass spectrometry analyses (TAP::SetA) 54

2.2.2.7. Protein stability assay using cycloheximide 55

2.2.3. Microscopy and quantification 55

2.2.3.1 Microscopic analyses $\quad 55$

2.2.3.2. Quantification methods 56

2.2.4. Analyses of sterigmatocystin production 56

\begin{tabular}{ll}
3. & Results \\
\hline
\end{tabular}

3.1. The Neddylation network in Aspergillus nidulans 57

3.1.1. DenA as NeddH E3 ligase 57

3.1.1.1. DenA interacts with the neddylation machinery in vivo 57

3.1.1.2. Deletion of $d c n A$ allows normal development while deletion of $r b x A$ is lethal $\quad 58$

3.1.1.3. DenA functions as an auxiliary NeddH-E3 ligase 59

3.1.2. The CSN deneddylase stabilizes Fbox15 60

3.1.3. The NeddH-associated proteins of unknown function AN4149 and AN4491 and fungal development 63

3.2. The sumoylation network in A. nidulans 64

3.2.1. The SumO protein of A. nidulans 64

3.2.1.1. Deletion of sumO delays asexual development 65

3.2.2. SumO isopeptidases 67

3.2.2.1. The SumO isopeptidase UlpA is required for fungal development 67

3.2.2.2. Crosstalk between the Sumo and NeddH fungal network 69

3.2.2.3. UlpB is a SumO-processing enzyme 71

3.2.3. Members of the SumO network and the sumoylation pathway 72

3.2.3.1. Identification of members of the SumO network 72

3.2.3.2. The E1 activation and E2 conjugation enzymes of the SumO
pathway are required for multicellular development in A. nidulans

3.2.4. The COMPASS complex controls development of Aspergillus nidulans 
3.2.4.1. SetA is part of an A. nidulans COMPASS complex 81

3.2.4.2. SetA is required for sexual development 83

3.2.4.3. SetA is required for proper conidiophore formation 84

3.2.4.4. SetA overexpression allows normal development 88

\begin{tabular}{ll}
$4 . \quad$ Discussion & 89 \\
\hline
\end{tabular}

4.1. The neddylation network of Aspergillus nidulans 89

4.1.1. DcnA promotes cullin neddylation 89

4.1.2. The COP9 signalosome counteracts the accumulation of developmental SCF complexes $\quad 91$

4.1.3. The NeddH associated proteins AN4491 and AN4149 are dispensable for A. nidulans development 92

4.2. The SumO network of Aspergillus nidulans 93

4.2.1. The SumO network and multicellular development 95

4.2.2. The SumO network and other UBL pathways 98

$\begin{array}{lll}\text { 4.2.3. The SumO network and gene expression } & 101\end{array}$

$\begin{array}{ll}\text { 4.3. Outlook } & 108\end{array}$

\begin{tabular}{ll} 
5. Literature & 112 \\
\hline
\end{tabular}

$\begin{array}{ll}\text { Abbreviations } & 130\end{array}$

Table of figures $\quad 134$

List of tables $\quad 136$

$\begin{array}{ll}\text { Acknowledgements } & 137\end{array}$

$\begin{array}{ll}\text { Curriculum vitae } & 138\end{array}$ 


\section{Summary}

Proteins can be post-translationally modified by the attachment of members of the ubiquitin-like protein family which requires the action of three enzymatic steps. The protein becomes activated by an E1 enzyme, transferred to an E2 enzyme and bound to the target with the help of E3 ligases. The modification of substrates with ubiquitin-like proteins can be reversed by isopeptidases. Nedd8 and Sumo are members of this protein family. Both proteins are conserved in the model organism Aspergillus nidulans. Whereas deletion of the nedd8 homolog neddH leads to cell death, the fungus can survive SumO deficiency but displays defects in sexual and asexual development. In this work, the two-component NeddH E3 ligase DcnA/RbxA was investigated. DcnA displays in vivo interactions with the neddylation machinery. Deletion of the gene leads to a moderate reduction of cullin neddylation levels but does not have consequences on fungal development. The RING finger protein RbxA has ligase activity towards NeddH and ubiquitin. A deletion of the corresponding gene leads to cell death. In a previous study with a strain deficient in the NeddH isopeptidase CSN, developmental relevant substrate adaptors of the SCF ubiquitin E3 ligase complexes (Fbox proteins) were identified. In this work it was found that the biochemical enrichment of Fbox 15 was not due to a general stabilization of the protein but likely to an arrest of a subportion of an Fbox15 containing SCF complex. In addition, the process of sumoylation was investigated in A.nidulans. Only a small subpopulation of proteins is sumoylated under normal growth conditions. To enrich SumO modified proteins, the genes for the two SumO isopeptidases UlpA and UlpB were deleted. Biochemical experiments in wild type and an UlpA deficient strain lead to the identification of a complex SumO network. This includes besides the sumoylating enzymes (E1, E2 and E3), histone modifying enzymes and complexes, transcriptional regulators, proteins involved in RNA maturation or stress response, as well as cross-talk with the processes of ubiquitination and neddylation. An interface of sumoylation and histone modification is the COMPASS complex which is involved in histone methylation. To better understand the role of the complex in the regulation of fungal development, the core subunit SetA was deleted. The resulting strain displayed defects in early sexual development, colony growth and secondary metabolism. Additionally, SetA is important for proper positioning of the asexual spore producing units. 


\section{Zusammenfassung}

Proteine können post-translational durch Ubiquitin und Ubiquitin-ähnliche Proteine modifiziert werden. Dies erfordert die Aktivität dreier Enzyme. Das Protein wird durch ein E1 Enzym aktiviert, an ein E2 Enzym übertragen und im letzten Schritt mit der Hilfe von E3 Ligasen kovalent an das Substrat gebunden. Dieser Prozess der posttranslationalen Modifikation ist reversibel durch Isopeptidasen. $\mathrm{Zu}$ der Proteinfamilie gehören unter anderem Sumo und Nedd8. Beide Proteine sind im Modellorganismus Aspergillus nidulans konserviert. Während die Deletion des nedd8 Homologs neddH zum Zelltod führt, können Pilze ohne SumO überleben. Diese Stämme weisen jedoch Defekte in der sexuellen und asexuellen Entwicklung auf. In dieser Arbeit wurde die NeddH E3 Ligase DcnA/RbxA untersucht. DcnA interagierte mit der Neddylierungs-Maschinerie und die Deletion des Gens führte zu einer leichten Reduktion der Neddylierung von Cullinen. Diese verminderte Neddylierung hatte jedoch keine Auswirkungen auf die pilzliche Entwicklung unter Laborbedingungen. Das RING-finger Protein RbxA zeigt eine E3 Ligaseaktivität sowohl in der Ubiquitinierung als auch in der Neddylierung. Eine Deletion des betreffenden Gens führte zum Zelltod. In einer vorangegangenen Studie mit einem Stamm mit Defekt in der Isopeptidase CSN wurden Substratadaptoren des SCF Ubiquitin-E3-Ligase Komplexes (Fbox-Proteine) identifiziert. In dieser Arbeit wurde festgestellt, dass die biochemische Anreicherung von Fbox 15 nicht auf eine generelle Stabilisierung des Proteins zurückzuführen ist, sondern wahrscheinlich auf eine Stabilisierung des SCF Komplexes mit Fbox15. Zusätzlich wurde der Prozess der Sumoylierung in A. nidulans untersucht. Unter normalen Wachstumsbedingungen ist nur ein kleiner Anteil der zellulären Proteine sumoyliert. Um diesen zu erhöhen, wurden die zwei SumO Isopeptidasen UlpA und UlpB untersucht. Durch biochemische Experimente im Wildtyp und einem Stamm, welchem die Isopeptidase UlpA fehlt, konnte ein komplexes SumO Netzwerk identifiziert werden. Zu diesem gehören neben den sumoylierenden Enzymen (E1, E2 und E3), Histon modifizierende Enzyme/Enzymkomplexe, andere Transkriptionsregulatoren, Proteine, die eine Rolle in der RNA-Reifung oder Stressantwort spielen, sowie Wechselwirkungen mit den Prozessen der Ubiquitinierung und Neddylierung. Eine wichtige Schnittstelle zwischen Sumoylierung und Histonmodifikation könnte hierbei der COMPASS Komplex sein. Dieser Komplex ist involviert in 
Histonmethylierung und damit in die Regulation der Transkription. Um ein besseres Verständnis für die Rolle des Komplexes in der Regulation der pilzlichen Entwicklung zu bekommen, wurde die Kerneinheit SetA deletiert. Der resultierende Stamm zeigte Defekte in der frühen sexuellen Entwicklung, im Koloniewachstum und Sekundärmetabolismus. SetA wurde als wichtiger Faktor für die richtige Positionierung der asexuellen Sporenträger identifiziert. 


\section{Introduction}

\subsection{Protein modification by members of the ubiquitin-like protein family}

\subsubsection{Post-translational modification of proteins}

Cells regulate the amount of proteins at different time points during their lives. This regulation can occur at different levels, for example, the modification of gene expression or the translation of RNA. In addition, the control can occur after translation of the protein. This latter process is referred to as post-translational modification of proteins. The function, structure, localization, stability or interactions of proteins can be modified by the covalent linkage of small functional groups or proteins. Examples for these modifications are phosphorylation, methylation or acetylation but also the attachment of proteins of the ubiquitin-like protein family (van der Veen \& Ploegh, 2012). This group of proteins is named after ubiquitin which is expressed ubiquitously in eukaryotes, the name giving characteristic of the polypeptide. Nedd8, Sumo, Fat10, Urm1 and other proteins compose the family of ubiquitin-like proteins (see chapter 1.1.2.). This study focuses on SumO and NeddH in the filamentous fungus Aspergillus nidulans.

\subsubsection{The family of ubiquitin-like proteins}

Ubiquitin was first described in 1975 (Goldstein et al., 1975). In 1980 it was for the first time connected to proteolysis (Ciechanover et al., 1980, Hershko et al., 1980). Meanwhile it is known that the attachment of ubiquitin to a substrate can have different effects on the protein. Whereas the formation of ubiquitin chains via its internal lysine 48 leads to proteasomal degradation, chains linked via its lysine 63 attached to a substrate or mono-ubiquitination of targets can have an impact on transcription, endocytosis, DNA repair, membrane protein trafficking and signal transduction, respectively (Hochstrasser, 2009).

The family of ubiquitin-like proteins consists of post-translational modifiers which only share small amino acid but high structural homology (Dye \& Schulman, 2007). The different members of this family have strong influence on many cellular processes and proteins. The ubiquitin-like protein Nedd8 (Neuronal precursor cell developmentally down-regulated gene 8) (Kumar et al., 1992, Kumar et al., 1993) is involved in the regulation of ubiquitin-dependent protein degradation (Merlet et al., 
2009) (see also chapter 1.2.2). The attachment of the ubiquitin-like protein Sumo (́mall ubbiquitin-like modifier) can have a strong impact on many cellular processes, such as DNA damage repair (Dou et al., 2011), transcription (Gill, 2005) and nucleoplasmic transport (Matunis et al., 1996) (see also chapter 1.3.). Different from ubiquitin and Nedd8, Sumo peptides have an additional amino acid extension at the N-terminus (Figure 1).

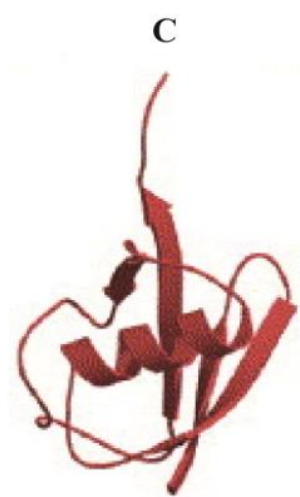

$\mathbf{N}$

Ubiquitin

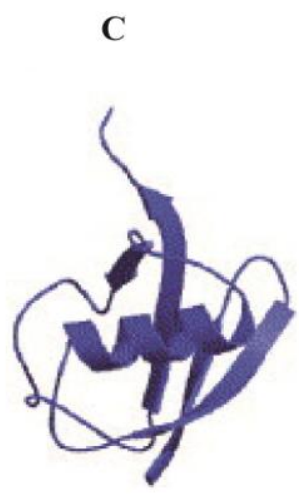

$\mathbf{N}$

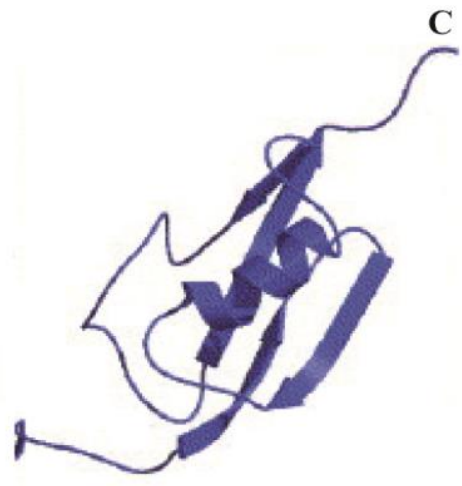

$\mathbf{N}$

Nedd8

Sumo1

\section{Figure 1: Structures of ubiquitin-like family proteins.}

Ribbon diagrams of human ubiquitin, Nedd8 and Sumo1, three members of the ubiquitin-like protein family that share high structural similarities. Reprinted from (Pickart \& Eddins, 2004) with permission from Elsevier.

Besides these well-known representatives of the ubiquitin-like protein family, there are other members like Fat10 (HLA- $\underline{F}$ adjacent transcript 10), Isg15

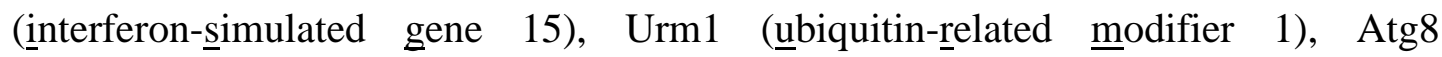
(autophagy related gene 8) and Atg12. Fat10 is only present in vertebrates. Its expression is specifically induced in immune defense (van der Veen \& Ploegh, 2012) and it can function as a proteasomal degradation signal (Buchsbaum et al., 2012). Atg8 is also important for protein degradation. The modification with this protein does not induce proteasomal degradation but autophagy. Additionally, Atg8 was shown to be connected with intracellular trafficking (Shpilka et al., 2011). Urm1 is involved in the process of tRNA modification (Leidel et al., 2009). Isg15 is only expressed in higher eukaryotes and has a role in immune response (Lenschow, 2010, Loeb \& Haas, 1992). 
An additional common feature of ubiquitin-like proteins is the similar but distinct attachment pathway of the modifiers to their respective targets (van der Veen \& Ploegh, 2012). It is characterized by three steps catalyzed by enzymes specific for the modifier. In a first energy-dependent step, the ubiquitin-like protein becomes activated by the action of an E1 enzyme. Next, it is conjugated to an E2 enzyme. The formation of a stable isopeptide bond between a C-terminal di-glycine motif of the modifier and the target substrate is often supported by specific E3 ligases. The modification reaction usually is reversible by the action of specific isopeptidases (van der Veen \& Ploegh, 2012).

\subsection{Nedd8 and the control of ubiquitin dependent protein degradation}

\subsubsection{Ubiquitin dependent protein degradation via the $26 \mathrm{~S}$ proteasome}

The strictly regulated degradation of proteins within a cell is an important process to remove misfolded polypeptides or proteins with temporary functions, involved in diverse cellular processes, such as circadian rhythm, immune and stress response, as well as DNA repair (Baud \& Derudder, 2011, Flick \& Kaiser, 2012, Henriques et al., 2009). In contrast, a misregulation of protein degradation can be involved in diseases such as cancer (Frankland-Searby \& Bhaumik, 2012). Two main pathways exist in eukaryotes to degrade proteins: autophagy and the degradation via the $26 \mathrm{~S}$ proteasome system. The target of autophagy can be unspecific cytoplasmic contents or specific proteins and organelles, depending on cellular stimuli. Within the process of autophagy, proteins are enclosed by a double membrane and degraded through fusion with lysosomes (Kundu \& Thompson, 2008).

Target substrates for proteasomal degradation are usually marked with chains of ubiquitin (Hochstrasser, 2009). The first ubiquitin binds to a lysine residue within the substrate. Further rounds of ubiquitination lead to chain formation at internal lysine residues of the first ubiquitin. Ubiquitin is expressed as a precursor protein and to generate mature, monomeric protein, the action of a protease is required (Larsen $e t$ $a l .$, 1996). This enzymatic reaction makes it accessible for the activation by the E1 enzyme (McGrath et al., 1991) and subsequent conjugation to one of several E2 enzymes (Hochstrasser, 1996). The substrate specificity of the ubiquitination reaction is mediated by a large group of ubiquitin E3 ligases. Ubiquitin E3 ligases are protein complexes that can bind ubiquitin charged E2s as well as the substrate protein, thus 
mediating the formation of the isopeptide bond between ubiquitin and its target (Wilkinson, 2000) (Figure 2A). The family of ubiquitin E3 ligases consists of two main members: HECT (homologous to E6-associated protein $\underline{\mathrm{C}}$-terminus) domain and RING (really interesting new gene) finger domain ligases (Kerscher et al., 2006). A large and well-investigated group of the ubiquitin ligases is the family of cullin RING E3-ubiquitin ligases (CRL) (Petroski \& Deshaies, 2005). The main characteristic of this type of ligase is the cullin subunit which serves as scaffold for the complex. In mammals, eight members of the cullin family are described (Cul1Cul7 and Parc) (Sarikas et al., 2011) and Cul1-5 can form around 300 different CRL complexes (Duda et al., 2011).

The best characterized members of the CRLs are the Cullin1 based Skp1cullin-Fbox protein (SCF) ubiquitin ligases. These complexes are important factors for the proteolysis of cell cycle regulators (Nakayama \& Nakayama, 2005). Cul1 binds the RING protein Roc1p/Rbx1p (regulator of çullins 1/ㅁING $\underline{b} \underline{\text { x }}$ protein 1 ) at its C-terminus which mediates the interaction with the E2 ubiquitin conjugation enzyme (Duda et al., 2011, Petroski \& Deshaies, 2005, Zheng et al., 2002). Skp1 (ㅁphase kinase-associated protein 1) functions as adaptor for various Fbox proteins at the N-terminus of Cull which are important for substrate specificity (Skowyra et al., 1997, Zheng et al., 2002). Fbox proteins can recognize their substrates by WD repeats and other interaction motifs (Merlet et al., 2009). Since the ubiquitin proteasome system has to deal with an immense variety of substrates, a high amount of Fbox proteins is needed. The number differs from approximately 70 Fbox proteins in human to around 700 Fbox domain containing proteins in A. thaliana (Gagne et al., 2002, Jin et al., 2004).

\subsubsection{Nedd8 and the control of cullin RING E3-ubiquitin ligases}

\subsubsection{Neddylation of cullin RING ligases}

The regulation of E3 ubiquitin ligases is a critical process in eukaryotic cells as misregulated protein degradation is connected to diseases such as cancer (Frankland-Searby \& Bhaumik, 2012), Parkinson's disease (Tan et al., 2009) and Alzheimer's disease (Riederer et al., 2011). The activity of CRLs is affected by covalent attachment and detachment of the ubiquitin-like protein Nedd8 (Merlet et al., 2009) (Figure 2B). Neddylation of the cullin scaffold results in enhancement of 
the ubiquitination reaction and stabilizes the CRL-E2 interaction (Saha \& Deshaies, 2008). This positive effect is based on an increase in ubiquitin-E2 recruitment and stimulation of the ubiquitin transfer mediated by conformational changes of the E3 ligase complex (Duda et al., 2008, Merlet et al., 2009).

A

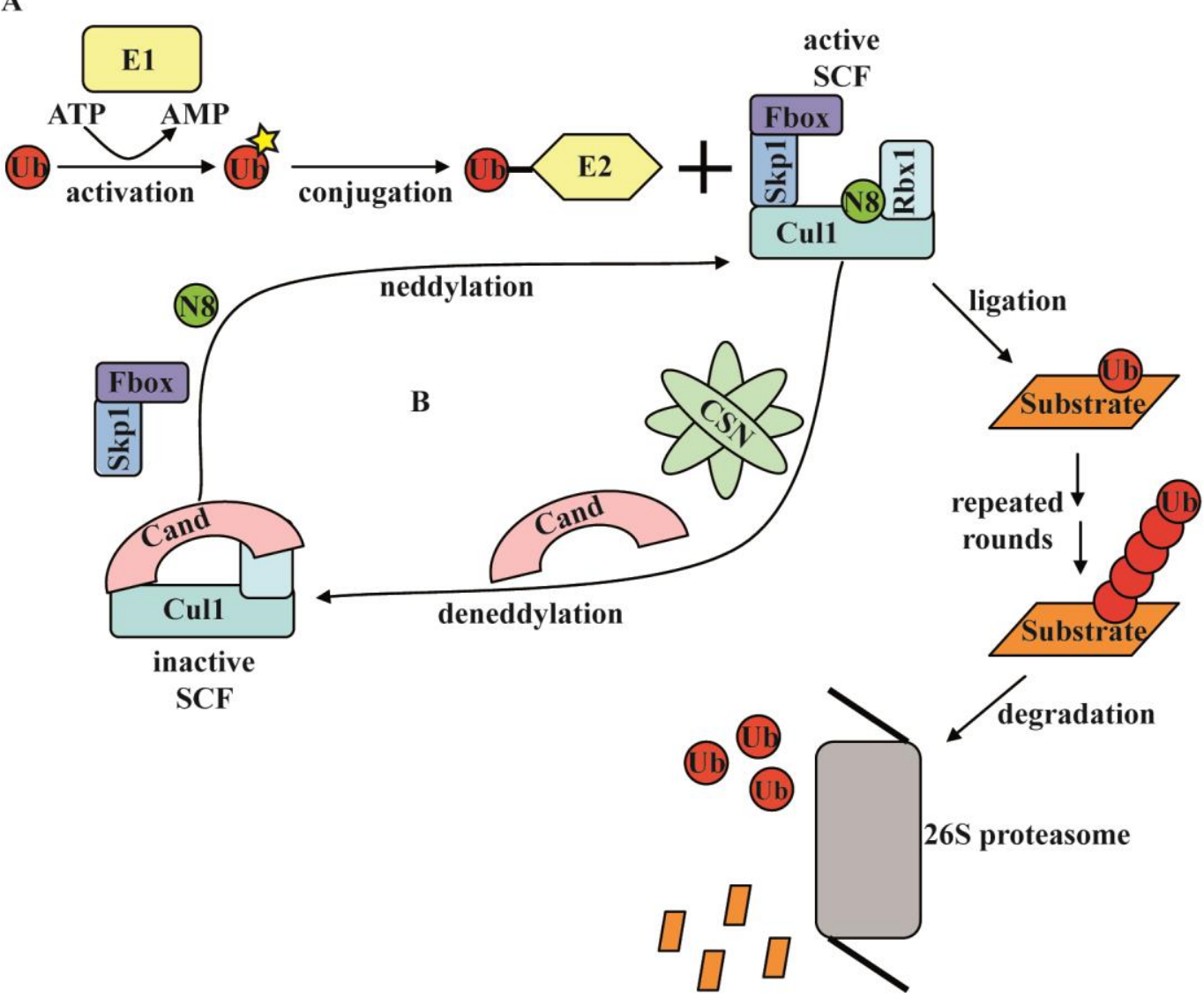

Figure 2: The ubiquitination reaction.

A Monomeric ubiquitin ( $\mathrm{Ub}$ ) becomes activated in an energy dependent step by the action of an E1 enzyme. Next, it is transferred to an E2 conjugation enzyme. The SCF complex is an E3 ubiquitin ligase. In its active form, it catalyzes the ligation of ubiquitin to its target substrate. By repeated rounds of the ubiquitination process, the substrate becomes polyubiquitinated. The marked protein is then recognized by the $26 \mathrm{~S}$ proteasome and becomes degraded, while ubiquitin is accessible for another ubiquitination reaction. B The SCF E3 ubiquitin ligase is active when the cullin subunit is modified with the small ubiquitin-like protein Nedd8 (N8). The complex becomes inactive by the action of the deneddylase CSN. Deneddylation facilitates the binding of Cand and leads to the dissociation of the Skp1/Fbox substrate adaptor. Inactive SCF complexes can be activated by the reattachment of a Skp1/Fbox substrate adaptor and the neddylation of Cul1 (for details see chapters 1.2.2.2. and 1.2.2.3.). 
Nedd8, similar to all members of the ubiquitin-like protein family, becomes attached to the cullin by an enzymatic cascade. The Nedd8 precursor protein becomes processed at the $\mathrm{C}$-terminus to make the di-glycine motif accessible (van der Veen \& Ploegh, 2012). The attachment of mature Nedd8 to the cullin is catalyzed by the Ula1/Uba3 E1 activating enzyme in yeast. Ubc12 acts as E2 enzyme in the neddylation process (Liakopoulos et al., 1998). E3 Nedd8 ligase activity has been attributed to the RING finger proteins (Dharmasiri et al., 2003, Morimoto et al., 2003, Scott et al., 2010) which are bound to the cullin subunit of CRLs as well as Dcn1 (defective in cullin neddylation-1) (Kurz et al., 2008, Kurz et al., 2005, Scott et al., 2010). Dcn1 positively effects the kinetics of the neddylation process (Kurz et al., 2005).

Den1 contains two domains: an ubiquitin-binding UBA domain and a socalled PONY (potentiating neddylation) domain (Figure 3). The UBA domain is located at the N-terminus and consists of three helices which are typical for UBA containing proteins. Mutation of the UBA domain does not affect the neddylation reaction (Kurz et al., 2008). The PONY domain is located at the C-terminus of Den1. It has a superhelical arrangement and contains a conserved Asp-Ala-Asp patch (composed of D226, A253 and D259), mediating the interaction with cullins (Kurz et al., 2008).

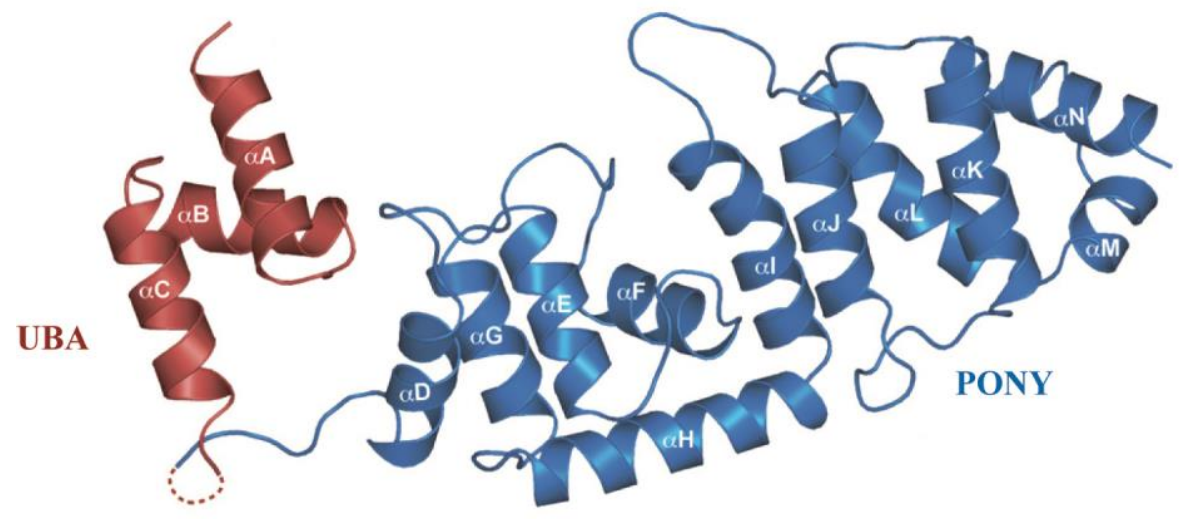

Figure 3: Structure of Den1p.

Ribbon structure of S. cerevisiae Dcn1p. The UBA domain is shown in red and the PONY domain in blue. Reprinted from (Kurz et al., 2008) with permission from Elsevier.

In addition, the crystal structure of the protein suggests the formation of antiparallel dimers in a head to tail conformation with an interaction of the UBA 
domain with the PONY domain of the second Den1. In vitro experiments confirmed the dimer formation (Kurz et al., 2008). Dcn1 can bind the cullin of the CRL and the E2 enzyme Ubc12 which in addition is bound to Rbx1. With this binding it can mediate the transfer of Nedd8 onto the cullin (Rabut \& Peter, 2008).

\subsubsection{Deneddylation of cullins by the COP9 signalosome}

The attachment of the ubiquitin-like protein Nedd8 to cullins activates SCF complexes that can then function as E3 ligases in the ubiquitination pathway (Saha \& Deshaies, 2008). This process, the neddylation reaction, is strictly regulated and reversible by deneddylating enzymes. The best studied deneddylase is a conserved protein complex, the $\underline{\mathrm{COP}} \underline{\underline{s}}$ ignalosome (CSN). It was first described in plants as constitutive photomorphogenic (COP) complex, according to the phenotypes of respective mutants (Wei \& Deng, 1992). The CSN consists of eight subunits (Csn1 to Csn8) and has a molecular weight of approximately $450 \mathrm{kDa}$. The COP9 signalosome is essential for viability in different organisms (Freilich et al., 1999, Tomoda et al., 2004, Wei \& Deng, 1999).

The CSN belongs to the multiprotein complexes of the PCI family. This family includes the regulatory LID of the proteasome, the $\underline{\mathrm{CSN}}$ as well as eIF3 (eukaryotic translation initiation factor 3) (PCI). All three share structural similarities, as each of them contains six subunits with a PCI domain (Enchev et al., 2010). This conserved domain is a universal binding domain for complex formation and recruitment of interaction partners (Scheel \& Hofmann, 2005). The PCI domain is composed of a winged helix and tetratricopeptide-like bihelical repeats (Pick et al., 2009). Besides the PCI domain proteins, the PCI family complexes contain a second type of subunit with a distinct conserved motif which is the MPN (Mprlp, Pad1p $\underline{\text { N- }}$ terminal) domain. The approximately 140 amino acids of this domain form an $\alpha / \beta$ fold (Hofmann \& Bucher, 1998) and can contain a so-called JAMM (Jab1/MPN domain metalloenzyme) motif. The MPN domain containing Csn5 is the catalytically active subunit of the COP9 signalosome which mediates the deneddylation of cullins (Cope et al., 2002).

Investigation of the CSN structure suggested a subunit organization similar to the arrangements of the subunits of the proteasomal LID and eIF3 (Enchev et al., 2010, Kapelari et al., 2000, Sharon et al., 2009). A model was suggested in which the PCI domain containing subunits interact mostly with their winged helix domains 
at the C-terminus. Csn5 and Csn6 in contrast are located on the outside of the planar PCI domain protein complex (Figure 4) (Enchev et al., 2012). The CSN can function as an interaction platform with other proteins. The complex can interact with deubiquitinating enzymes such as Usp15, transcriptional regulators and kinases (Wei et al., 2008).
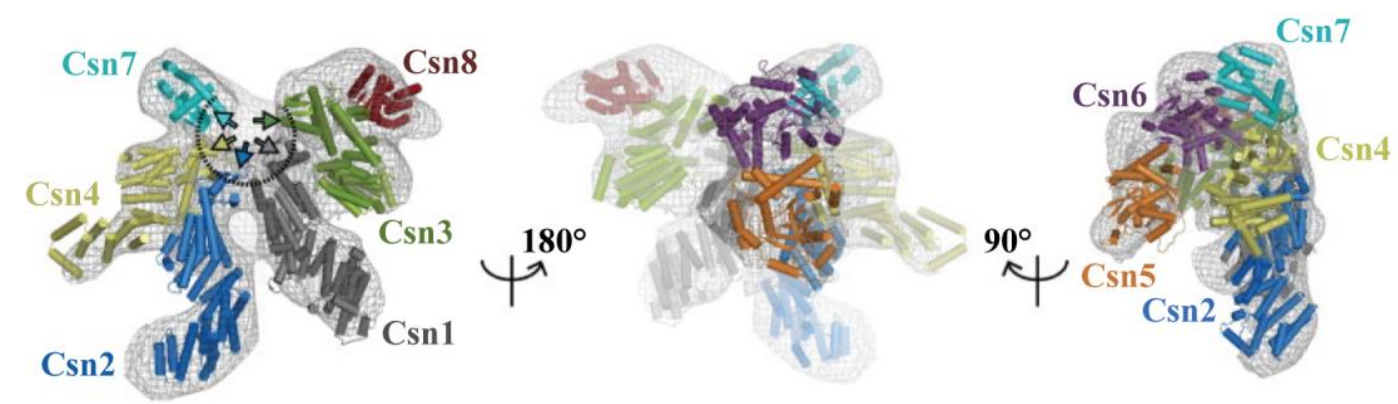

Figure 4: Model of the architecture of the COP9 signalosome.

The subunits of the complex were modeled into an electron microscopy map (grey mash). Left: potential organization of the PCI domain subunits with arrows showing the approximate coplanar positions. Middle: model of the complex from the other side, showing the MPN domain subunits Csn6 (purple) and Csn5 (orange). Right: model of the complex from the side. Reprinted from (Enchev et al., 2012) with permission from Elsevier.

\subsubsection{Modification of cullin by Cand1}

CRLs remain in an active state while they are modified with Nedd8 and become inactivated through deneddylation by the CSN. In the inactive state, the CRL disassembles which allows the exchange of the substrate adaptor subunits (Wei et al., 2008). Another level of CRL activity regulation is the binding of Cand1 (cullinassociated nedd8-dissociated protein 1). Cand proteins of different organisms bind deneddylated cullins (Bosu et al., 2010, Chuang et al., 2004, Helmstaedt et al., 2011, Hwang et al., 2003, Liu et al., 2002). Cand1 can be encoded by a single polypeptide or as a two genes as in A. nidulans. The two Cand proteins together form fungal CandA (Helmstaedt et al., 2011). Crystal structures revealed that Cand1 forms a 2pronged clamp. The C-terminus of Cand1 thereby binds to the substrate adaptor site of Cul1, while the N-terminal part blocks the neddylation site (Duda et al., 2011, Goldenberg et al., 2004).

In detail, the Cand cycle is thought to start with the deneddylation process, making inactive subcomplexes of the CRL accessible. Cand 1 can then bind to cullin- 
RING subcomplexes, thus regulating the exchange of the substrate adaptor (Liu et al., 2002). When new Skp1-Fbox substrate adaptors are available, Cand1 dissociates from the cullin (Siergiejuk et al., 2009). Next, the CRL can be activated by neddylation again and fulfill its function as E3 ubiquitin ligase.

\subsubsection{The deneddylase Den 1}

The second protein in human which has a deneddylating activity is Den1. Originally annotated as a Sumo-specific isopeptidase, in vitro studies showed that it has a significant high affinity to Nedd8 (Gan-Erdene et al., 2003). Besides the deneddylation activity, this protein is also responsible for processing of the immature Nedd8 protein (Gan-Erdene et al., 2003, Wu et al., 2003). Deletion of the Den1 ortholog Nep1p and its related enzyme Nep2p in $S$. pombe had only little effect on the yeast cells (Zhou \& Watts, 2005). Recent studies showed that mammalian Den1 and Aspergillus DenA interact with the CSN complex, suggesting an interplay between the two deneddylases. The major interacting subunit changed during evolution, as in human Den1 predominantly interacts with Csn1, whereas in A. nidulans the interacting subunit is CsnG (corresponding to human Csn7). Additionally, the stability of Den 1 and DenA is controlled by the COP9 signalosome (Christmann et al., 2013).

\subsection{The small ubiquitin-like modifier Sumo}

Sumo belongs to the family of ubiquitin-like proteins. It displays high structural similarity to ubiquitin, although it only shares approximately $20 \%$ sequence identity on the level of primary protein sequence (Bayer et al., 1998). Sumo was described as a member of the ubiquitin-like protein family in 1996 when it was identified to interact with mammalian proteins (Geiss-Friedlander \& Melchior, 2007). For example, it was found to modify RanGAP1. Whereas RanGAP1 is localized in the cytoplasm, the modified form was found at the nuclear pore complex (Matunis et al., 1996).

In the model organism S. cerevisiae only one Sumo protein, named Smt3p, is present. Other organisms express several Sumo isoforms. In the genome of Arabidopsis thaliana eight functional Sumo proteins are encoded (Kurepa et al., 2003). In mammals, four Sumo proteins are expressed. Sumo1-3 are present 
ubiquitously (Geiss-Friedlander \& Melchior, 2007). Sumo4 was identified as a tissue-specific protein, expressed in kidney, lymph knots and spleen (GeissFriedlander \& Melchior, 2007, Guo et al., 2004). Sumo2 and Sumo3 share high sequence homology of approximately $95 \%$, whereas Sumo1 is around $45 \%$ identical (Wang \& Dasso, 2009). Sumo4 is approximately $86 \%$ identical to Sumo2, but is only insufficiently studied. The mammalian Sumo proteins show differences in their subcellular localization and substrate specificity (Ayaydin \& Dasso, 2004, Vertegaal et al., 2006).

Sumo proteins can form chains via the linkage of an internal conserved lysine residue which is present in Sumo2, Sumo3 and yeast Smt3p (Bylebyl et al., 2003, Tatham et al., 2001). Sumo1, in contrast, is missing such a lysine residue and therefore might act as the last element of a chain composed of Sumo2 and Sumo3 (Matic et al., 2008), although Sumo1 chains were described in vitro (Pichler et al., 2002). The biological function of Sumo chains is not fully understood. In S. pombe a lack of the lysines 14 and 30, which are important for chain formation, leads to cellular defects (Skilton et al., 2009). In contrast, mutation of the N-terminal lysines in yeast Smt3p did not have strong effects (Bylebyl et al., 2003).

\subsubsection{Consequences of sumoylation}

Many proteins have been suggested to be modified by Sumo proteins, therefore sumoylation can influence diverse cellular processes. In general, sumoylation of a target protein can result in three different consequences (Figure 5). First, the Sumo protein can disturb interactions of the substrate with other proteins or DNA. Second, sumoylation can enable new interactions, for example by recruiting binding partners with SIMs (see chapter 1.3.3.). Third, the conformation of a substrate can change upon the attachment of Sumo (Geiss-Friedlander \& Melchior, 2007).

It is known that the modification with Sumo can have an impact on transcription through sumoylation of transcriptional activators or repressors and corepressors (Zhao, 2007). In general, the modification with Sumo is more connected to a repressive function in transcriptional control (Gill, 2005). It is suggested that low-level sumoylation of transcriptional regulators often results in the binding of additional factors that either form an inhibitory complex or lead to chromatin remodeling, thus resulting in efficient transcriptional repression (Geiss-Friedlander \& 
Melchior, 2007). Important chromatin remodelers involved in Sumo-dependent gene repression are histone deacetylases (Gill, 2005). In contrast to these inhibitory functions of sumoylation on transcription, there are several examples of Sumo modifications activating transcription (Lyst \& Stancheva, 2007).

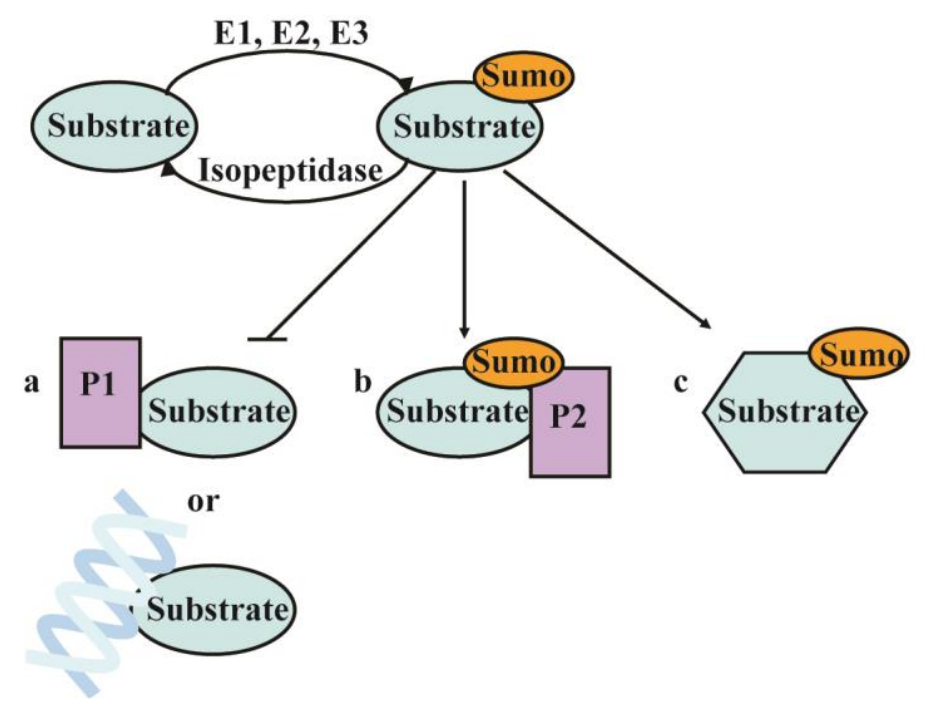

Figure 5: Possible consequences of sumoylation for substrate proteins.

Sumo molecules are attached to target substrates by the action of an enzymatic cascade of E1, E2 and E3 enzymes. This process is reversible by Sumo-specific isopeptidases. The consequences of Sumo modifications can in general be (a) an inhibition of interactions of the target and other proteins (P1) or DNA, (b) the formation of interactions with new partners (P2), for example through SIMs or (c) a conformational change of the target substrate. Modified after (Geiss-Friedlander \& Melchior, 2007).

Sumoylation also functions in DNA repair (Dou et al., 2011). One example is the sumoylation of the DNA repair enzyme Thymidine DNA glycosyltransferase (TDG) which enables a conformational change and release of the enzyme from DNA (Baba et al., 2005, Hardeland et al., 2002). Additionally, sumoylation is linked to nuclear in- and export (Melchior et al., 2003), chromosome assembly and segregation as well as development (Zhao, 2007).

Another important issue is the interaction of Sumo with ubiquitin. Proteins can be modified at one lysine residue with either Sumo or ubiquitin, resulting in diverse effects for the target and leading to competitive situations (Praefcke et al., 2012). Additional interactions of the two pathways are also possible by the attachment of one modifier to the enzymes of the pathway of the other modifier. Some enzymes can also function in both pathways. Cross-talk between the systems

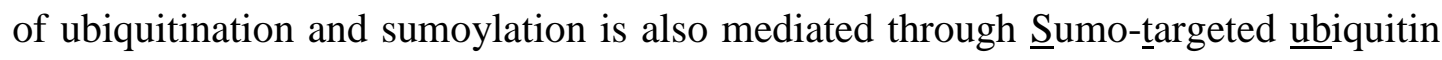


ligases (STUbLs) which are conserved in eukaryotes (Praefcke et al., 2012). In yeast the STUbLs Hex3p (Slx5p)/Slx8p and Ris1p (Uls1p) were identified (Uzunova et al., 2007, Xie et al., 2007). STUbLs can recognize sumoylated proteins through SIM motifs and attach ubiquitin. The ubiquitin can serve as proteasomal degradation signal leading to proteolysis of the Sumo modified substrate (Uzunova et al., 2007). STUbLs are important for genome stability and other cellular pathways (Garza \& Pillus, 2013). Besides the described cellular functions of Sumo proteins, sumoylation is also involved in various diseases, making the understanding of sumoylation and its regulation an important field of research. In several cancer types, an increased expression of Sumo proteins and sumoylating enzymes was found. In addition, protooncogenic proteins and tumor suppressors are sumoylated (Zhao, 2007). Furthermore, sumoylation is linked to neurodegenerative diseases as for example multiple system atrophy, Huntington's, Alzheimer's and Parkinson's disease. Sumo was found in inclusions typical for these diseases or sumoylation of proteins directly involved in these disorders has been shown, such as Tau or $\alpha$-Synuclein (Dorval \& Fraser, 2007). Knowledge about Sumo modifications also gains importance in cardiac function and pathology (Wang, 2011) and Sumo4 is connected to diabetes. In particular, one point mutation (M55V) in Sumo4 was identified which led to an increased risk for diabetes (Wang \& She, 2008). During viral infections, it was shown that sumoylation of viral proteins promotes the infection and leads to a decrease of host cell protein sumoylation (Zhao, 2007).

\subsubsection{The Sumo attachment pathway}

Similar to other ubiquitin-like proteins, Sumo is expressed as an immature precursor protein that becomes processed by a Sumo-specific hydrolase (Melchior et al., 2003). This process uncovers the di-glycine motif at the C-terminus which is essential for the attachment process. In S. cerevisiae, Smt3p, the yeast Sumo protein, is activated in an ATP-dependent manner by an E1 heterodimer consisting of the two proteins Aos $1 p$ (activation of $\underline{\operatorname{S} m t} 3 \mathrm{p} 1$ ) and Uba2p (Ubiquitin activating 2). Aos $1 \mathrm{p}$ resembles the N-terminal part of the E1 Ubiquitin activating enzyme Uba1p, whereas Uba2p shares similarity with its C-terminal end (Johnson et al., 1997). In the activation step, a thioester bond is formed between Uba2p and Smt3p (Johnson et al., 1997). Then, the Sumo protein is transferred to the E2-conjugation enzyme, Ubc9p, through a trans-thiolation reaction (Johnson \& Blobel, 1997). In contrast to the 
ubiquitination reaction where different E2 enzymes were identified, Ubc9p is the only Sumo E2 enzyme (Wilkinson \& Henley, 2010). Structural studies of a complex of Ubc9 and the Sumo substrate RanGAP1 showed that the enzyme can recognize a sumoylation motif (see chapter 1.3.3) of the substrate protein (Bernier-Villamor et $a l ., 2002)$. Sumo is bound to a free amino group of a lysine residue in this motif by the formation of an isopeptide bond. This last step of the sumoylation reaction is often facilitated by the action of specific E3 ligases. In S. cerevisiae, many proteins become Sumo modified by the action of Siz1p and Siz2p, two proteins of the family of Siz/PIAS (protein inhibitor of activated $\underline{\text { STAT }}$ (signal transducer and activator of transcription)) RING finger-like domain proteins (Johnson \& Gupta, 2001, Takahashi et al., 2001a, Takahashi et al., 2001b). Proteins of this family usually share a common organization with five domains. The first one is the SAP (scaffold attachment factor-A/B, acinus and PIAS) domain which is usually localized at the $\mathrm{N}$ terminus. It contains a LxxLL motif, which is present in proteins that interact with nuclear receptors. The second domain is named PINIT (Proteasome subunits, Int-6, Nip-1, and TRIP-15) which corresponds to the PCI domain. This motif is important for the localization of the protein. The SP-RING domain is a conserved structure consisting of a cysteine-rich region and is located in the center part of the protein. It mediates protein-protein interactions and exhibits the E3 ligase activity. It is followed by SIMs and a variable S/T-rich region (Rytinki et al., 2009) (Figure 6).

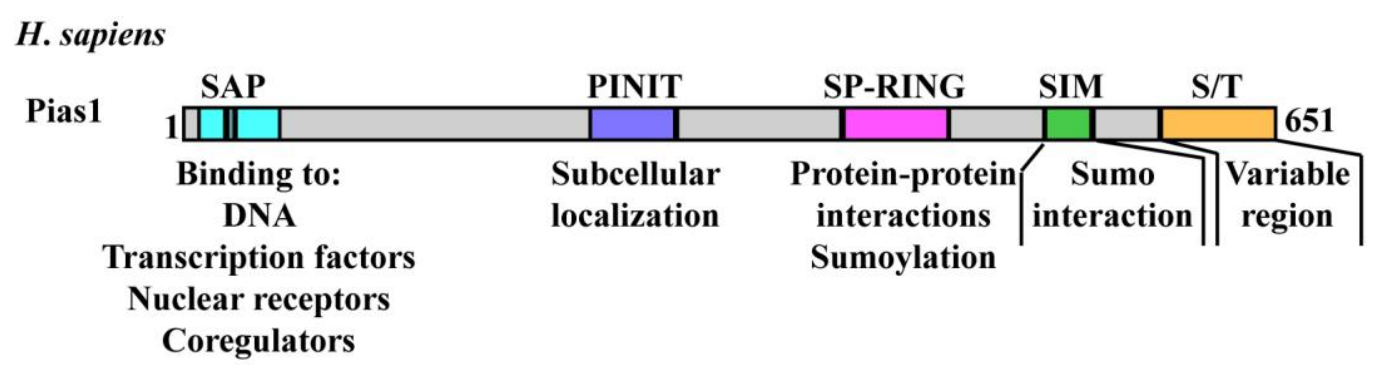

S. cerevisiae

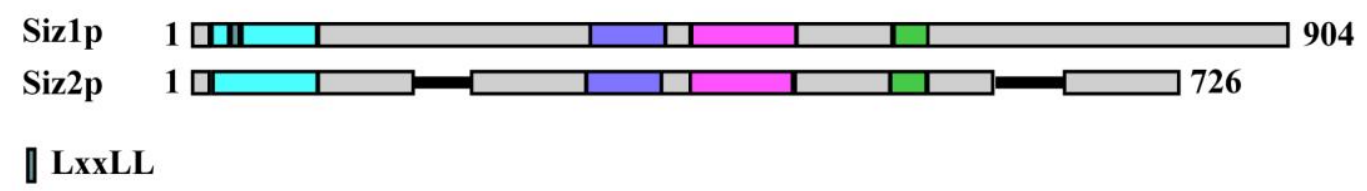

\section{Figure 6: Domain structure of Siz/PIAS proteins.}

Schematic domain organization of human Pias1, Siz1p and Siz2p from S. cerevisiae. All three proteins function as Sumo E3 ligases and show a similar protein structure. Functions of the domains are shown. Numbers indicate amino acids. Modified after (Rytinki et al., 2009). 
In addition to the Siz proteins, two other Sumo E3 ligases were identified in yeast: Cst9p (Zip3p) (Cheng et al., 2006) and Mms21p (Zhao \& Blobel, 2005). Cst9p plays a role in synaptonemal complex formation during meiosis (Cheng et al., 2006). Mms21p is a subunit of a nuclear protein complex. Deletion of MMS21 leads to an increased sensitivity against DNA damage stress and defects in nucleolar integrity and telomere functions (Zhao \& Blobel, 2005). In vertebrates the function of Sumo E3 ligases is attributed to five PIAS domain proteins (Palvimo, 2007) but also additional proteins, such as RanBP2 (Pichler et al., 2002, Pichler et al., 2004).

\subsubsection{The Sumoylation motif}

The attachment sites of Sumo are usually composed of a short amino acid motif: $\psi \mathrm{KxE}(/ \mathrm{D})$, with $\psi$ as a large, hydrophobic and $\mathrm{x}$ being any amino acid (Rodriguez et al., 2001). This motif can also be reversed (Matic et al., 2010). Additionally, extended variations of the four amino acid core motif were published (Table 1). The PDSM ( Phosphorylation-dependent sumoylation motif) was found in transcriptional regulators and heat shock proteins. It comprises of the sumoylation motif, followed by a proline-directed phosphorylation site (Hietakangas et al., 2006). The second addition of the Sumo motif is NDSM (negatively charged amino aciddependent sumoylation motif) which is composed of the core motif and additional acidic residues within the next 10 amino acids (Yang et al., 2006). Another variant of an extended Sumo motif is the connection of the core motif with additional hydrophobic amino acids, called HCSM (hydrophobic cluster sumoylation motif) (Matic et al., 2010).

Table 1: Sumoylation motifs.

\begin{tabular}{|c|c|}
\hline Motif & Consensus sequence \\
\hline Sumo consensus sequence $^{\mathrm{a}}$ & $\psi \mathrm{KxE}(/ \mathrm{D})$ \\
\hline Reversed consensus sequence $^{b}$ & $(\mathrm{D} /) \mathrm{ExK} \psi$ \\
\hline Phosphorylation-dependent sumoylation motif $^{\mathrm{c}}$ & $\psi \mathrm{KxE}(/ \mathrm{D}) \mathrm{xxSP}$ \\
\hline $\begin{array}{l}\text { Negatively charged amino acid-dependent } \\
\text { sumoylation motif }\end{array}$ & $\psi \mathrm{KxE}(/ \mathrm{D}) \mathrm{xx}(\mathrm{D} / \mathrm{E})_{4}$ \\
\hline Hydrophobic cluster sumoylation motif $^{\mathrm{e}}$ & $\psi \psi \psi \mathrm{KxE}(/ \mathrm{D})$ \\
\hline
\end{tabular}


Sumo can also interact to other proteins without the covalent linkage to a

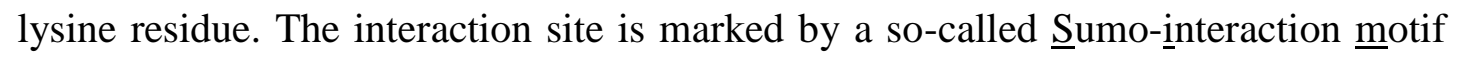
(SIM). SIMs often contain a hydrophobic core but do not have a defined amino acid sequence. The interactions of SIMs with other proteins are important for the Sumo attachment reaction and also for the modification of proteins (Kerscher, 2007).

\subsubsection{Desumoylation}

The process of sumoylation can be reversed by specific proteases. In yeast there are two Ubiquitin-like protein-specific proteases (Ulp) known, whereas in human six Sentrin/Sumo-specific proteases (Senp) have been described. In addition to their desumoylation activity, Sumo proteases have the important function to process the precursor Sumo protein making it attachable to other proteins (Wang \& Dasso, 2009, Yeh, 2009). Ulps/Senps have a conserved catalytic domain of approximately 200 amino acids which is located at the C-terminus (Wang \& Dasso, 2009).

An overview about the Senps/Ulps is given in table 2. The two Ulps in yeast localize to different compartments and fulfill specific functions. ULPI is essential for viability (Li \& Hochstrasser, 1999), whereas deletion of ULP2 leads to defects in chromosome segregation, growth and sporulation (Li \& Hochstrasser, 2000). Ulp1p is localized at the nuclear envelope. One but not the only function of the protein is the processing of Smt3p as expression of the mature Sumo protein weakly complements an ULP1 deletion (Li \& Hochstrasser, 1999). Ulp2p is localized in the nucleoplasm (Li \& Hochstrasser, 2000) and it cleaves poly-Smt3p chains (Bylebyl et $a l ., 2003)$. A third protein with Sumo isopeptidase function in yeast was discovered, which is Wss1p (weak suppressor of $\underline{s} \mathrm{mt} 3$ 1). It cleaves both, Sumo-Ubiquitin as well as Sumo chains in vitro, although the efficiency in the cleavage of Sumo chains was lower compared to Ulp2p (Mullen et al., 2010).

The six mammalian Senps (Senp1-3 and Senp5-7) can be divided into two groups. Whereas Senp1-3 and Senp5 more resemble Ulp1p, Senp6 and Senp7 are similar to Ulp2p (Mukhopadhyay \& Dasso, 2007). Senp1 and Senp2 localize to the nuclear envelope and the nuclear pore/nuclear specles, respectively. They show processing and deconjugation activity for all three human Sumo proteins. The other Senp proteases display specificity for Sumo2 and Sumo3. Senp3 and Senp5, which are present in the nucleoli, are important for deconjugation. In contrast, Senp6 and 
Senp7 are localized to the nucleoplasm and are involved in the cleavage of Sumo chains (Yeh, 2009, Wang \& Dasso, 2009).

Table 2: Human Senps and yeast Ulps. Modified after (Yeh, 2009).

\begin{tabular}{|c|c|c|c|}
\hline Name & Localization & Specificity & Activity \\
\hline Senp1 & Nuclear envelope & Sumo1/2/3 & $\begin{array}{c}\text { Processing and } \\
\text { deconjugation }\end{array}$ \\
\hline Senp2 ${ }^{\text {a }}$ & Nuclear pore/specles & Sumo1/2/3 & $\begin{array}{c}\text { Processing and } \\
\text { deconjugation }\end{array}$ \\
\hline Senp3 $^{\text {a }}$ & Nucleoli & Sumo2/3 & Deconjugation \\
\hline Senp5 $^{\text {a }}$ & Nucleoli & Sumo2/3 & Deconjugation \\
\hline Senp6 $^{\text {a }}$ & Nucleoplasm & Sumo2/3 & Cleavage of chains \\
\hline Senp7 $^{\text {a }}$ & Nucleoplasm & Sumo2/3? & Cleavage of chains? \\
\hline Ulp1p $^{\text {b }}$ & Nuclear envelope & Smt3p & $\begin{array}{c}\text { Processing and } \\
\text { deconjugation }\end{array}$ \\
\hline Ulp2p $^{\text {c }}$ & Nucleoplasm & Smt3p & Cleavage of chains \\
\hline Wss1p & Nuclear envelope & $\begin{array}{c}\text { Smt3p } \\
\text { Cleavage of chains }\end{array}$ \\
\hline
\end{tabular}

\subsection{The model organism Aspergillus nidulans}

Fungi of the genus Aspergillus belong to the group of ascomycetes and can be isolated from soil. As saprophytes, they can use a wide range of materials as nutrition sources. Aspergilli are of significant importance as specific species are of medical, scientific and industrial relevance (Baker \& Bennett, 2007). For instance, A. flavus and A.fumigatus are opportunistic human pathogens that cause severe infections in immune-compromised individuals, such as HIV, organ transplant or cancer patients in chemo- or radiotherapy (Hedayati et al., 2007, McCormick et al., 2010). In addition, A. flavus and A. parasiticus produce a secondary metabolite, named Aflatoxin, contaminating spoiled food (Georgianna \& Payne, 2009). Industrially, Aspergilli are applied for the production of a number of enzymes, acids, food ingredients and antibiotics (Knuf \& Nielsen, 2012).

In natural sciences, A. nidulans is a model organism for genetics, morphology, metabolism and cell biology intensively studied since the 1950's (Pontecorvo et al., 1953). Its genome is fully sequenced and published (Galagan et al., 2005). It has a size of approximately $30 \mathrm{Mb}$, distributed among eight chromosomes and encodes more than 8000 genes. A. nidulans is a well established genetic system, allowing a broad range of research. 


\subsubsection{Vegetative growth}

Vegetative development of A. nidulans starts from dormant mitotically or meiotically derived spores (see chapter 1.4.2. and 1.4.3.). The nucleus of an asexual spore, or both nuclei in sexual spores, is arrested in the G1 phase (Bergen \& Morris, 1983). Before changing to polarized growth, the spore swells and a nuclear division occurs (Harris, 1999). The forming germe tube grows by apical extension which requires the deposition of cell wall material at the hyphal tip. Within the growing hyphae, nuclear divisions and hyphal branching takes place, leading to the formation of a fungal mycelium (Harris, 2007). Upon reaching a defined cell size, a septum is generated within the hyphae (Wolkow et al., 1996). A small pore in the septum allows the passage of cytoplasm and organelles. These pores can be plugged by socalled Woronin bodies in case of hyphal damage to prevent the loss of protoplasm from the complete mycelium by sealing of the septum within the destroyed area (Jedd \& Pieuchot, 2012). After 16-20 h of vegetative growth, the mycelium reaches developmental competence which enables the fungus to respond to external stimuli (Adams et al., 1998) and induce asexual or sexual development (Axelrod et al., 1973) (Figure 7).

\subsubsection{Sexual reproduction}

In the absence of light and under elevated carbon dioxide conditions, competent mycelium of $A$. nidulans reduces conidiophore formation (see chapter 1.4.3.) and enters the sexual life cycle (Braus et al., 2002). As a homothallic fungus, it can complete sexual reproduction without the presence of a mating partner. This sexual phase of the organism is also referred to as Emericella nidulans (Braus et al., 2002, Pontecorvo et al., 1953). Sexual reproduction is important to survive during suboptimal environmental conditions. The formation of the sexual fruit body, the cleistothecium, starts with the fusion of two ascogonial hyphae that form a dikaryon enclosed by the nest-like growing mycelium. The name "nidulans" is derived from this characteristic, as it means "nest-former" (Pontecorvo et al., 1953). Thick-walled cells are formed, the so-called Hülle cells. These cells provide tissue specific enzymes and were suggested to nurse the developing fruit body (Sarikaya Bayram et al., 2010). 


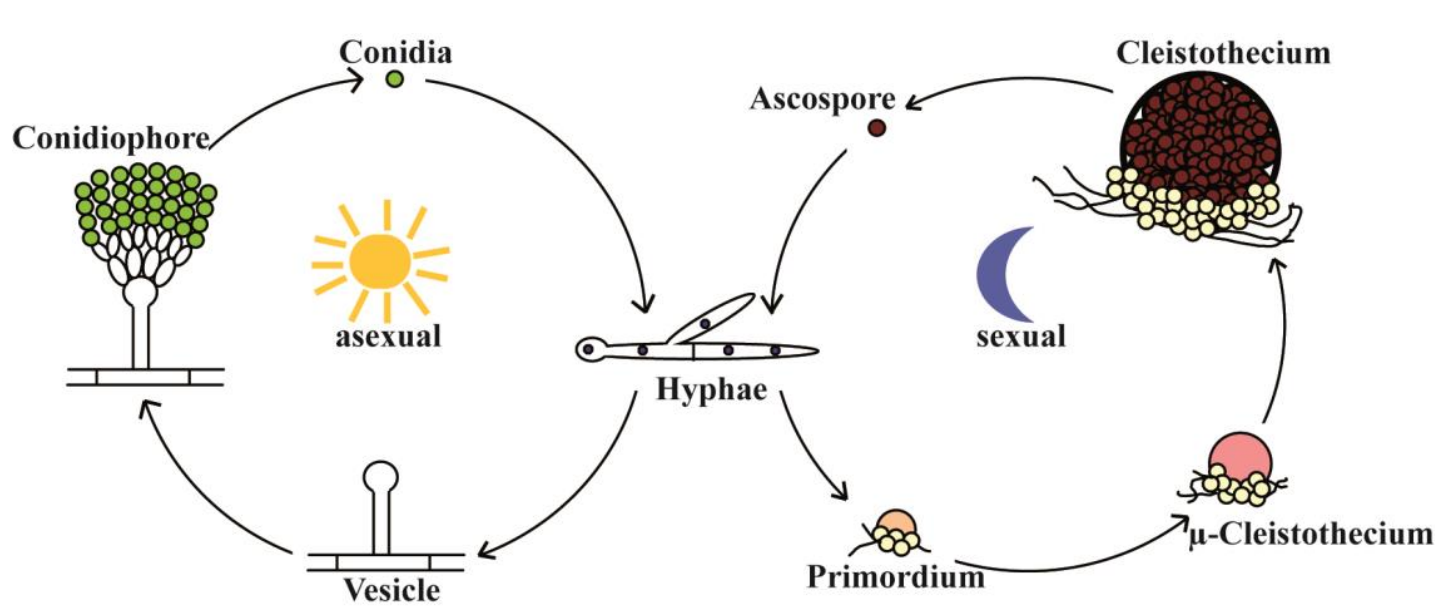

Figure 7: Life cycle of Aspergillus nidulans.

Asexually or sexually derived spores of $A$. nidulans germinate and form vegetative hyphae. From this, depending on environmental conditions, the fungus enters the asexual (left) or sexual (right) life cycle. In light, the asexual cycle starts with the formation of a conidiophore vesicle. In the next step the conidiophore is developed. Synchronic budding at the vesicle surface leads to the formation of metulae. The phialides are formed at the distal tips of the metulae by another budding event. By constriction of the phialide tips, asexual spores, the conidia, are developed which are released into the air (Etxebeste et al., 2010, Park \& Yu, 2012, Timberlake, 1990). In darkness the sexual cycle is favored. A primordium is formed which is surrounded by specialized tissue and Hülle cells, nursing the developing fruit body. The primordium maturates to a microcleistothecium and further to a cleistothecium within seven days. The cleistothecium contains asci with eight binucleate ascospores each (Braus et al., 2002, Champe et al., 1994, Dyer \& O'Gorman, 2012).

In the nests, the primordium is formed that develops to an immature fruit body precursor, the microcleistothecium. The chleistothecial envelope is produced by the surrounding mycelia network glued together by a so far not further characterized substance Cleistin. In the developing cleistothecium, ascogenous hyphae develop into croziers. Dikaryotic cells are formed and fusion of the nuclei leads to the formation of diploid zygotes. One round of meiosis and a round of mitosis lead to the presence of eight nuclei in each ascus of the developing fruit body. Membrane formation separates the nuclei and supports the formation of the ascospores. A second round of mitosis completes the formation of eight binucleate spores per ascus. Within the wall of the fruit body, the red pigment asperthecin is accumulated. Every mature cleistothecium contains approximately 80.000 ascospores and has a diameter of 150-200 $\mu \mathrm{m}$ (Braus et al., 2002, Chae et al., 2007, Champe et al., 1994, Dyer \& O'Gorman, 2012, Laubinger, 2008). 


\subsubsection{Asexual reproduction}

Asexual development of $A$. nidulans is induced by the presence of light and at normal carbon dioxide partial pressure (Adams et al., 1998). It can be divided into five morphological steps (Timberlake, 1990). The development of the asexual structure, the conidiophore, starts with the formation of a foot cell from vegetative hyphae located at the interface of air and surface. From this cell an aerial hyphae is formed, the so-called stalk. This stalk grows to a length of approximately $100 \mu \mathrm{m}$. It does not contain any septa or branches. A multinucleated conidiophore vesicle is developed at its tip by terminal swelling. Next, budding at the vesicle surface leads to the formation of approximately 60 primary sterigmata, so-called metulae. One nucleus enters each metulae and a septum is formed to divide the vesicle and the newly formed cells. Two or three uninucleate secondary sterigmata, the phialides, are formed at the distal tip of each metulae by another budding event. Metulae and phialides are also separated by septa. At the phialides tips, the first round of asexual spores is developed. The nucleus of the phialide is localized to its top and undergoes mitotic division. The daughter nucleus is transported to the developing spore, while the second nucleus remains in the phialide. The conidium is separated from the phialide by septum formation. By repeated rounds of conidiospore formation, long chains of uninucleate asexual spores are formed (Etxebeste et al., 2010, Park \& Yu, 2012, Timberlake, 1990).

In the phialide cells, two genes, $w A$ and $y A$, are transcribed that are important for the green pigmentation. The gene product of $y A$, the conidial laccase, is secreted from the phialide cell and accumulates in the cell wall of the spore. Asexual spores are released into the air and can colonize new areas. The green pigmentation of the conidiospores functions as protection against UV damage (Mayorga \& Timberlake, 1990, Timberlake, 1990, O'Hara \& Timberlake, 1989).

\subsubsection{Regulation of development}

The production of developmental structures requires the temporal and spatial action of a number of proteins which have to be strictly regulated dependent on environmental factors. One of the most important regulators for A. nidulans development is light. For proper light response, the fungus requires a set of different receptor proteins (Bayram \& Braus, 2012). The red light receptor is encoded by fphA 
which displays similarity to bacterial phytochromes. It plays a role in the repression of sexual reproduction in red light (Blumenstein et al., 2005). LreA and LreB function in the detection of blue light (Purschwitz et al., 2008). In addition the only cryptochrome/photolyase protein of A. nidulans, CryA, acts as blue light sensor. Deletion of cryA leads to Hülle cell formation in vegetative culture conditions (Bayram et al., 2008a, Bayram \& Braus, 2012).

A well-studied protein for the connection of light sensing, development and secondary metabolism is VeA (Bayram \& Braus, 2012, Purschwitz et al., 2009, Yager, 1992). VeA interacts with FphA which in turn is connected to LreA and LreB (Purschwitz et al., 2008) (Figure 8). Additionally, CryA regulates veA expression (Bayram et al., 2008a). VeA shows light-dependent localization: in light, VeA is present in the cytoplasm and the nucleus, while in darkness it accumulates in the nucleus. The transport is mediated by the importin KapA (Figure 8) (Stinnett et al., 2007). Deletion of $v e A$ results in a block of sexual development, whereas overexpression leads to the production of high numbers of cleistothecia even in liquid culture and a reduced amount of asexual structures (Kim et al., 2002). Additionally, the veA deletion strain showed a reduction in the production of sterigmatocystin and penicillin (Kato et al., 2003).

VeA forms a dimer with the velvet-like protein VelB in the cytoplasm. in darkness, VelB is moved into the nucleus with VeA. Inside the nucleus the so-called velvet complex is formed between VeA, VelB and the methyltransferase LaeA (Bayram et al., 2008b) (Figure 8). LaeA is an important regulator for the production of secondary metabolites, as for example sterigmatocystin and penicillin, but is also important for proper light regulation (Bayram et al., 2008b, Bayram \& Braus, 2012, Bok \& Keller, 2004). Deletion of laeA causes a reduction of conidiospore formation as well as an increase of sexual development in strains with an intact veA gene. Additionally, laeA deletion results in a reduction of cleistothecia size due to a lack of nursing Hülle cells (Sarikaya Bayram et al., 2010). Deletion of velB leads to defects in sexual fruit body production and an altered secondary metabolism (Bayram et al., 2008b). In vegetative hyphae and during darkness, VelB forms a dimer with VosA

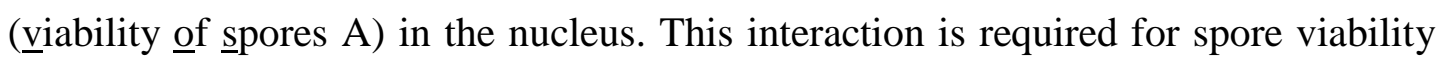
and the repression of asexual development (Sarikaya Bayram et al., 2010). 
The regulation of sexual development additionally includes a MAP kinase cascade with AnFus3. VeA becomes phosphorylated by AnFus3. In addition, AnFus3 promotes the interaction of VeA with VelB (Bayram et al., 2012).

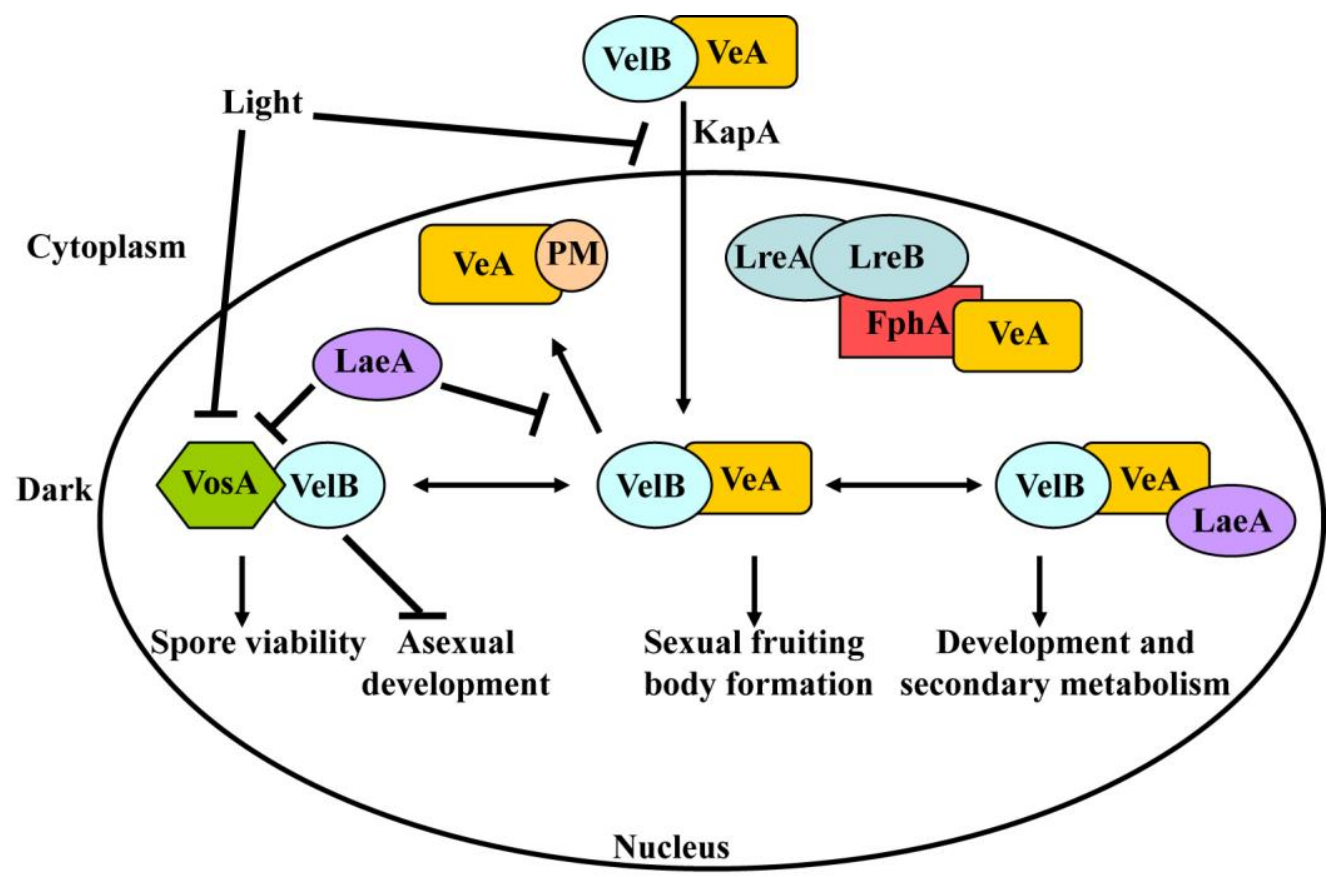

Figure 8: Light dependent regulation of development.

In darkness, the VelB/VeA dimer is imported into the nucleus, mediated by KapA. In the nucleus, it can form the velvet complex with the methyltransferase LaeA. This complex is involved in the regulation of development and secondary metabolism. VelB can also form a dimer with VosA which is important for the repression of asexual development and spore viability. VeA interacts with the red light receptor FphA which interacts with the blue light receptors LreA and LreB. VeA might undergo a posttranslational modification (PM). Light promotes asexual development by decreasing the levels of VosA/VelB. Modified from (Bayram \& Braus, 2012).

VeA also has an influence on the balance of cellular oxilipin levels by affecting the expression of the dioxygenase like enzyme PpoA. PpoA, PpoB and PpoC are involved in the biosynthesis of hormone-like signal molecules, also known as psi (precocious sexual inducer) factors. The ratio of these factors determines the induction of development. In the veA deletion strain, the ppoA expression is drastically reduced (Bayram \& Braus, 2012, Tsitsigiannis et al., 2004). PpoA and PpoC are additionally regulated in a CSN-dependent manner (Nahlik et al., 2010, Tsitsigiannis et al., 2004).

Several proteins have been identified which function in the signal transduction pathways of sexual reproduction. Among them NsdD (never in sexual 
development D), SteA (sterile 12-like A) and RcoA. Deletion of $n s d D$ results in a block of cleistothecia formation. In contrast, overexpression increases the number of fruit bodies and induces Hülle cell formation under vegetative growth conditions (Han et al., 2001). Also deletions in steA and rcoA lead to a block in sexual development (Todd et al., 2006, Vallim et al., 2000).

BrlA (bristle A) and AbaA (abacus A) are two transcription factors involved in the regulation of asexual development (Clutterbuck, 1969). BrlA is a zinc-finger protein important for the switch from apical growth to vesicle and sterigmata formation. brlA mutants are impaired in asexual development, as stalk formation is not affected but neither sterigmata nor spores are produced (Prade \& Timberlake, 1993). Overexpression of brlA leads to conidiophore development under vegetative growth conditions (Adams et al., 1988). BrlA itself activates several additional genes which are indispensable for conidiophore formation. Among those are $y A$ and $a b a A$ (Timberlake \& Clutterbuck, 1994). AbaA is important for phialide differentiation (Sewall et al., 1990) and potentiates its own transcription in a positive feedback-loop. Additionally, it induces the expression of wetA. In return, brlA transcription is repressed (Han \& Adams, 2001). WetA is a regulator for spore-specific genes. For example, it is involved in the production of cell wall components (Marshall \& Timberlake, 1991). Asexual development is regulated by a number of proteins. Several factors have been identified that are involved in the activation of conidiation and the transcription of $\operatorname{brlA}$. FluG is important for the inhibition of vegetative growth and the induction of brlA activation (Park \& Yu, 2012). Also VosA, which interacts with VelB in response to light, functions in asexual development. Deletion of vosA leads to constitutive conidiospore formation, even in liquid culture. Furthermore, the expression of $\operatorname{brl} A, y A$ and $w A$ is altered in the vosA deletion strain. VosA is also required for the synthesis of threalose which is important for the survival of spores (Ni \& Yu, 2007).

\subsubsection{Neddylation and deneddylation in A. nidulans}

In the filamentous fungus $A$. nidulans all proteins of the neddylation and ubiquitination machineries are conserved. The main substrates of the neddylation pathway are cullins. Cullins function as subunits in ubiquitin E3 ligases, such as SCF complexes. It was shown that several subunits of SCF complexes are indispensable for A. nidulans life: the ortholog of Skp1, SkpA, as well as the Cu1 ortholog CulA 
(von Zeska Kress et al., 2012). Fbox proteins serve as substrate specific adaptors for the protein degradation machinery. In A. nidulans 74 different Fbox or Fbox-like domain containing proteins were identified (Ö. Bayram, unpublished). Only few of these proteins have been described. GrrA is a Fbox protein specifically important for sexual reproduction in A. nidulans (Krappmann et al., 2006). SconB was identified as one of four proteins involved in sulphur metabolism (Natorff et al., 1993) and recently, FbxA was described to have a role in xylose metabolism (Colabardini et al., 2012). Neddylation regulates the activity of SCF E3 ligases (Duda et al., 2011). It was shown that the Nedd8 ortholog NeddH and the E2 conjugation enzyme UbcL are essential for fungal viability (von Zeska Kress et al., 2012). The neddylation process can be reversed by the deneddylating CSN. Whereas many fungi, as S.pombe, $N$. crassa or S. cerevisiae encode a partial CSN complex or even only single subunits, A. nidulans displays an eight subunit COP9 signalosome with all subunits of the mammalian complex conserved (Braus et al., 2010, Busch et al., 2007). Deletions in the CSN subunits result in viable strains that are blocked in sexual development at the stage of primordia formation, an altered secondary metabolism as well as changes on the transcriptome and proteome level (Braus et al., 2010, Busch et al., 2003, Busch et al., 2007, Nahlik et al., 2010). This makes the fungus a valuable model organism for the neddylation/deneddylation process. The second deneddylase and ortholog of Den1, is named DenA in A. nidulans. Like CSN, it is not essential for fungal viability but contrary to the deneddylating complex, deletions are not affecting sexual fruit body formation but asexual development and the light regulation of development. It was shown, that the protein does not cleave the precursor $\mathrm{NeddH}$ protein but displays deneddylation activity in the filamentous fungus (Christmann et al., 2013).

\subsubsection{The SumO protein of Aspergillus nidulans}

In the filamentous fungus A. nidulans, only one Sumo protein, named SumO, is encoded which is similar to yeast. In contrast, plants and vertebrates express several Sumo isoforms (Geiss-Friedlander \& Melchior, 2007). However, a striking

difference to S.cerevisiae Smt3p is that the SumO protein of A.nidulans is not essential for the organisms' viability (Wong et al., 2008). This makes the organism useful for research. SumO of A. nidulans shares a sequence homology with human 
Sumo1-3 of approximately 45\%. A model of the secondary structure displays high similarity to the human protein (Figure 9).

A sumO deletion strain of $A$. nidulans shows strong defects in asexual and sexual development (Wong et al., 2008). The production of asexual conidia is drastically reduced, although the conidiophore structure appears to be normal. Sexual development is blocked at the stage of microcleistothecia formation. In addition, colony growth on complete medium is disturbed and an increased sensitivity against stress inducing agents was reported (Wong et al., 2008). Sumoylation is also involved in the regulation of secondary metabolism (Szewczyk et al., 2008). Additional studies confirmed the block in sexual development and reduced stress tolerance, but could not report defects in colony formation on minimal medium. Furthermore, the fungus started to develop sexual fruit bodies during light and aeration, where normally asexual spore production is favored (Laubinger, 2008).

These data suggest that sumoylation in A. nidulans has a strong effect on many regulatory pathways, although specific substrates have not been described so far.

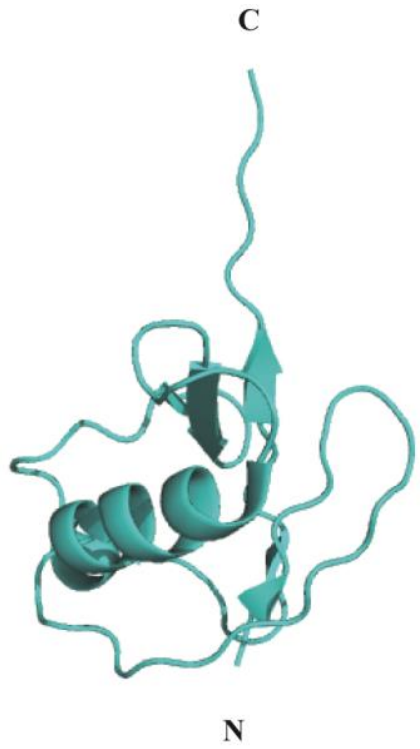

Sumo1

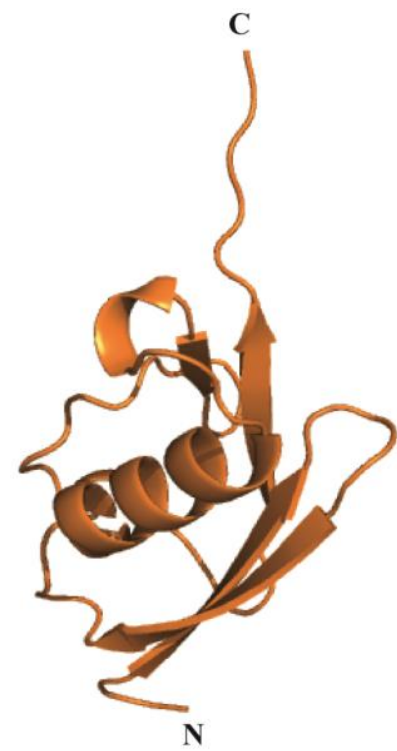

SumO

\section{Figure 9: The Aspergillus nidulans SumO protein in comparison to human Sumo1.}

Ribbon structure models of the human Sumo1 protein and Aspergillus nidulans SumO. The peptide sequence of SumO was modeled on the partial structure of Sumo1. 


\subsection{Scope and aim of this study}

The modification of proteins with the ubiquitin-like modifiers SumO and $\mathrm{NeddH}$ are important regulatory processes for A. nidulans growth, development and coordinated secondary metabolism (Laubinger, 2008, Szewczyk et al., 2008, von Zeska Kress et al., 2012, Wong et al., 2008). The first part of this study will address different aspects of the essential neddylation reaction. DcnA and RbxA have been suggested to function as E3 ligases in the transfer of Nedd8 onto the cullin (Scott et $a l ., 2010)$. The role of these putative E3 ligases in the neddylation reaction and for development of the model organism A. nidulans will be analyzed in this part of the study. Interactions of DenA and the neddylation machinery will be investigated and the function of the two proteins in development will be addressed by genetic studies. In a CSN deneddylase deficient strain NeddH co-purifies with developmental relevant F-box proteins, as for example Fbox15 (von Zeska Kress et al., 2012). This biochemical enrichment could be explained by two scenarios: first, the overall amount of Fbox15 becomes stabilized in the CSN deletion strain. Second, a subpopulation of the protein becomes arrested within the SCF complex and can be biochemically enriched. To address these possibilities, the influence of CSN towards Fbox 15 stability will be examined. Furthermore, two proteins of unknown function, AN4149 and AN4491, were identified as NeddH-associated but their role for fungal development is elusive (von Zeska Kress et al., 2012). Deletion strain construction followed by phenotypical characterization will be applied to investigate a putative role of AN4149 and AN4491 in the neddylation process.

In the second part of this work, the sumoylation network in A. nidulans will be analyzed, as targets for this modification in the fungus are not described so far. Using biochemical approaches, SumO modified or SumO-interacting proteins will be identified. As sumoylation is a reversible process, SumO-specific isopeptidases will be characterized to find conditions which allow an enrichment and identification of putative substrates. The SumO network within the fungal cell will be further analyzed by genetical approaches. The COMPASS complex displays a putative interface between sumoylation and histone modification and will be characterized in this second part of the thesis. A crucial point will be the role of the complex during Aspergillus development. 


\section{Materials and Methods}

\subsection{Materials}

\subsubsection{Growth media and growth conditions}

Chemicals for media, buffers and solutions were obtained from MERCK KGAA (Darmstadt, D), CARl Roth GmbH \& Co. KG (Karlsruhe, D), AplliChem GMbH (Darmstadt, D), Invitrogen GMBH (Karlsruhe, D), FluKa AnAlytiCAL (Neu-Ulm, D), Sigma-Aldrich Chemie GmbH (Steinheim, D), VWR InTERnATIONAL GMbH (Darmstadt, D), BD BiosciEnCES (Heidelberg, D) and SERVA ELECTROPHORESIS GMBH (Heidelberg, D).

\subsubsection{Escherichia coli}

E. coli strains were cultivated in LB medium ( $1 \%$ bacto-tryptone, $0.5 \%$ yeast extract, $1 \% \mathrm{NaCl}, \mathrm{pH} 7.5$, for solid medium: $2 \%$ agar) (Bertani, 1951) at $37^{\circ} \mathrm{C}, 30^{\circ} \mathrm{C}$ or $25^{\circ} \mathrm{C}$, respectively. For selection, $100 \mathrm{mg} / \mathrm{ml}$ ampicillin was added.

\subsubsection{Aspergillus nidulans}

Aspergillus nidulans strains were cultivated in or on minimal medium [MM, $1 \%$ glucose, $2 \mathrm{mM} \mathrm{MgSO} 4,70 \mathrm{mM} \mathrm{NaNO}_{3}, 7 \mathrm{mM} \mathrm{KCl}, 11.2 \mathrm{mM} \mathrm{KH}_{2} \mathrm{PO}_{4}$ (pH 5.5), 0.1\% trace element solution, for solid medium: 2\% agar] (Bennet \& Lasure, 1991) at $30^{\circ} \mathrm{C}$ or $37^{\circ} \mathrm{C}$. For TAP purifications complete medium $[0.5 \%$ yeast extract, $1 \%$ peptone, $7 \mathrm{mM} \mathrm{KCl}, 11.2 \mathrm{mM} \mathrm{KH}_{2} \mathrm{PO}_{4}$ (pH 5.5), $2 \mathrm{mM} \mathrm{MgSO}_{4}, 0.1 \%$ trace element solution, $2 \%$ agar] was used instead.

Strains, carrying nitrate promoter constructs, were grown in/on London Medium [1\% glucose, $2 \%$ salt solution $\left(26 \mathrm{~g} / \mathrm{l} \mathrm{KCl,} 26 \mathrm{~g} / 1 \mathrm{MgSO}{ }_{4}, 76 \mathrm{~g} / \mathrm{KH}_{2} \mathrm{PO}_{4}\right.$, $5 \%$ (v/v) trace elements) $\mathrm{pH} 6.5$ ] with $70 \mathrm{mM} \mathrm{NaNO}_{3}$ for induction, or $5 \mathrm{mM} \mathrm{NH}_{4}^{-}$ tartrate for repression, respectively. $2 \%$ agar was added for plates. Media were supplemented with $100 \mu \mathrm{M}$ pyridoxine- $\mathrm{HCl}, 5 \mathrm{mM}$ uridine and $5 \mathrm{mM}$ uracil as required (Käfer, 1977). For selection of strains with dominant markers the following chemicals were added to the medium: $10 \mu \mathrm{g} / \mathrm{ml}$ phleomycin (CAYLA-INVIVOGEN, Toulouse, F) for the ble marker gene of Streptoalloteichus hindustanus (Austin et al., 1990), $100 \mathrm{ng} / \mathrm{ml}$ pyrithiamine (TAKARA BIO INC., Münsing, D) for ptrA resistance 
cassette (Kubodera et al., 2000) and $100 \mathrm{ng} / \mathrm{ml}$ nourseothricin (ClonNAT; WERNER BIOAgENTS, Jena, D) for the NAT resistance cassette (Krugel et al., 1993).

Vegetative mycelia were harvested from liquid cultures inoculated with appropriate numbers of asexual spores in flasks with indentations and grown on a rotary shaker for $20 \mathrm{~h}$ at $30^{\circ} \mathrm{C}$ or $37^{\circ} \mathrm{C}$, respectively. For developmental induction, $1 \times 10^{6}$ spores were spread on agar plates. Asexual development was induced by incubation of the plates in constant white light whereas the sexual life cycle was triggered under oxygen limiting conditions on tape-sealed plates in the darkness.

\subsubsection{Strains, plasmids and primers}

\subsubsection{E. coli strains}

For general cloning procedures and purification of plasmid DNA E. coli DH5 $\alpha\left[\mathrm{F}^{-}, \Phi 80 \mathrm{~d} \Delta\right.$ (lacZ) M15 ${ }^{-1}, \Delta$ (lacZYA-argF) U169, recA1, endA1, hsdR17 (rK, $\mathrm{mK}^{+}$), supE44, $\lambda^{-}$, thi1, gyrA96, relA1] (Woodcock et al., 1989) was used.

\subsubsection{Plasmids used in this study}

All plasmids used in this study are listed in table 3. Detailed construction information is given below.

Table 3: Plasmids used in this study.

\begin{tabular}{|c|c|c|}
\hline Name & Description & Reference \\
\hline $\begin{array}{l}\text { pCR-BluntII- } \\
\text { TOPO }\end{array}$ & General cloning vector $\left(\mathrm{kan}^{R}\right)$ & $\begin{array}{l}\text { INVITROGEN GMBH } \\
\text { (Karlsruhe, D) }\end{array}$ \\
\hline $\begin{array}{l}\text { pJET } 1.2 \\
\text { blunt }\end{array}$ & General cloning vector $\left(a m p^{R}\right)$ & $\begin{array}{l}\text { FERMENTAS GMBH } \\
\text { (St. Leon-Rot, D) }\end{array}$ \\
\hline $\begin{array}{l}\text { pBluescript }{ }^{\circledR} \mathrm{I} \\
\text { I SK+ }\end{array}$ & General cloning vector $\left(a m p^{R}\right)$ & $\begin{array}{l}\text { STRATAGENE } \\
\text { (La Jolla, CA, USA) }\end{array}$ \\
\hline pME2968 & pGEM@-5Zf(+) with ntap & (Busch et al., 2007) \\
\hline pME3005 & $\begin{array}{l}\text { neddH cDNA in pBluescript }{ }^{\mathbb{B}} \mathrm{II} \\
\text { SK+ }\end{array}$ & (von Zeska Kress et al., 2012) \\
\hline pME3012 & $\begin{array}{l}\text { N-terminal half of eyfp (BiFC) in } \\
\text { pMCB17apx }\end{array}$ & (Blumenstein et al., 2005) \\
\hline pME3013 & $\begin{array}{l}\text { C-terminal half eyfp (BiFC) in } \\
\text { pMCB17apx }\end{array}$ & (Blumenstein et al., 2005) \\
\hline pME3024 & $\begin{array}{l}\text { A. oryzae ptrA resistance gene in } \\
\text { pBluescript }{ }^{\circledR} \text { II SK+ }\end{array}$ & (Krappmann et al., 2006) \\
\hline pME3160 & $\begin{array}{l}\text { niiA/niaD bidirectional promoter } \\
\text { AfpyrG }\end{array}$ & (Bayram et al., 2008b) \\
\hline
\end{tabular}


Table 3: Plasmids used in this study, continued.

\begin{tabular}{|c|c|c|}
\hline Name & Description & Reference \\
\hline pME3281 & $\begin{array}{l}\text { pBluescript }{ }^{\circledR} \text { II SK+ with } \\
\text { phleomycin resistance cassette }\end{array}$ & (Helmstaedt et al., 2011) \\
\hline pME3671 & cDNA $r b x A$ in pJET1.2/blunt & (von Zeska Kress et al., 2012) \\
\hline pME3672 & cDNA $d c n A$ in pJET1.2/blunt & (von Zeska Kress et al., 2012) \\
\hline pME3673 & $d c n A:: N-e y f p$ in pME3160 (PmeI) & (von Zeska Kress et al., 2012) \\
\hline pME3674 & C-eyfp::rbxA in pME3673 (SwaI) & (von Zeska Kress et al., 2012) \\
\hline pME3675 & $\begin{array}{l}\text { C-eyfp:: neddH in pME3673 } \\
(\text { SwaI })\end{array}$ & (von Zeska Kress et al., 2012) \\
\hline pME3676 & C-eyfp::ubcL in pME3673 (SwaI) & (von Zeska Kress et al., 2012) \\
\hline pME3677 & C-eyfp in pME3673 (SwaI) & (von Zeska Kress et al., 2012) \\
\hline pME3678 & cDNA $u b c \mathrm{~L}$ in $\mathrm{pJET} 1.2 / \mathrm{blunt}$ & (von Zeska Kress et al., 2012) \\
\hline pME3928 & Cassette tap::nat in pJET1.2/blunt & (Christmann et al., 2013) \\
\hline pME3929 & Cassette $g f p::$ nat in pJET1.2/blunt & (Christmann et al., 2013) \\
\hline pME3950 & $f b x 15:: s g f p$ in pME3856 (PmeI) & (von Zeska Kress et al., 2012) \\
\hline pME3967 & $\begin{array}{l}5^{\prime} \text { and } 3^{\prime} \text { flanking region of sumO } \\
\text { in pME2968 (ApaI/NdeI) }\end{array}$ & This study \\
\hline pME3968 & $\begin{array}{l}\text { sumO cDNA::terminator::pyrG in } \\
\text { pME3967 (ClaI) }\end{array}$ & This study \\
\hline pME3976 & $\begin{array}{l}\text { Deletion cassette } u b a B \text { (pyrG } \\
\text { marker with flanking regions of } \\
u b a B \text { ) in pJET } 1.2 / \text { blunt }\end{array}$ & This study \\
\hline pME3977 & $\begin{array}{l}\text { Deletion cassette aosA (ptrA } \\
\text { marker with flanking regions of } \\
\text { aosA) in pJET1.2/blunt }\end{array}$ & This study \\
\hline pME3978 & $\begin{array}{l}\text { Deletion cassette sizA (ptrA } \\
\text { marker with flanking regions of } \\
\text { sizA)in pJET1.2/blunt }\end{array}$ & This study \\
\hline pME3979 & $\begin{array}{l}\text { Deletion cassette } u l p A \text { (pyroA } \\
\text { marker with flanking regions of } \\
u l p A \text { ) in pJET1.2/blunt }\end{array}$ & This study \\
\hline pME3984 & $\begin{array}{l}\text { Deletion cassette } u b c N \text { (ptrA } \\
\text { marker with flanking regions of } \\
u b c N \text { ) in pJET1.2/blunt }\end{array}$ & This study \\
\hline pME3985 & $\begin{array}{l}\text { 5' flanking region::ulpA::3' } \\
\text { flanking region in } \mathrm{pME} 3281 \\
(\text { EcoRV) }\end{array}$ & This study \\
\hline pME3986 & $\begin{array}{l}\text { 5' flanking } \\
\text { region::ubaB::terminator in } \\
\text { pJET1.2/blunt }\end{array}$ & This study \\
\hline pME3987 & $\begin{array}{l}\text { pyroA }(A f):: 3^{\prime} \text { flanking region } \\
u b a B \text { in } \mathrm{pME} 3986(\text { StuI })\end{array}$ & This study \\
\hline pME3994 & $\begin{array}{l}\text { Complementation cassette aosA } \\
(\text { aosA }(p):: \text { aosA::aosA }(t):: \text { pyroA }:: \\
\text { 3'flanking region) in } \\
\text { pJET1.2/blunt }\end{array}$ & This study \\
\hline
\end{tabular}


Table 3: Plasmids used in this study, continued.

\begin{tabular}{|c|c|c|}
\hline Name & Description & Reference \\
\hline pME3995 & $\begin{array}{l}\text { Complementation cassette } u b c N \\
(u b c N(p):: u b c N:: u b c N(t):: \text { pyroA: } \\
: \text { 3'flanking region) in } \\
\text { pJET1.2/blunt }\end{array}$ & This study \\
\hline pME3996 & $\begin{array}{l}\text { Deletion cassette } \operatorname{set} A \text { ( } p t r A \\
\text { marker with flanking regions of } \\
\text { setA) in pJET1.2/blunt }\end{array}$ & This study \\
\hline pME3997 & $\begin{array}{l}5^{\prime} \text { flanking region::setA:: } 3^{\prime} \\
\text { flanking region in } \mathrm{pME} 3281 \\
(\text { EcoRV })\end{array}$ & This study \\
\hline pME4064 & $\begin{array}{l}\text { Deletion cassette } u l p B \text { (ptrA } \\
\text { marker with flanking regions of } \\
u l p B \text { ) in pJET1.2/blunt }\end{array}$ & This study \\
\hline pME4065 & $\begin{array}{l}\text { Deletion cassette } A N 4149 \text { (ptrA } \\
\text { marker with flanking regions of } \\
\text { AN4149)in pJET1.2/blunt }\end{array}$ & This study \\
\hline pME4066 & $\begin{array}{l}\text { Cassette tap::setA in } \\
\text { pJET1.2/blunt }\end{array}$ & This study \\
\hline pME4067 & $\begin{array}{l}5^{\prime} \text { flanking region::ulpB:: } 3^{\prime} \\
\text { flanking region } \mathrm{pME} 3281 \\
(\text { EcoRV })\end{array}$ & This study \\
\hline pME4068 & gDNA denA in pME3160 (SwaI) & This study \\
\hline pME4069 & $\begin{array}{l}\text { Deletion cassette } A N 4491 \text { (ptrA } \\
\text { marker with flanking regions of } \\
\text { AN4491) in pJET1.2/blunt }\end{array}$ & This study \\
\hline pME4070 & setA $:: g f p$ in pME3160 $(S w a \mathrm{I})$ & This study \\
\hline
\end{tabular}

Af: Aspergillus fumigatus, $\mathrm{t}$ : terminator, $\mathrm{p}$ : promoter

\subsubsection{Primers used in this study}

All oligonucleotides used in this study are listed in table 4. Primers were purchased from EUROFINS MWG OPERON (Ebersberg, D).

Table 4: Primers used in this study.

\begin{tabular}{|l|l|l|}
\hline \multicolumn{1}{|c|}{ Name } & Size & \multicolumn{1}{c|}{ Sequence } \\
\hline JS1 & 21mer & 5'-TGA CTT CGT CGT TAA GGG TTC-3' \\
\hline JS2 & $41 \mathrm{mer}$ & $\begin{array}{l}\text { 5'-TAA CCA ATG GGA TCC CGT AAT CGC GAT } \\
\text { GCT TAT TGT GGC GT-3' }\end{array}$ \\
\hline JS3 & $41 \mathrm{mer}$ & $\begin{array}{l}\text { 5'-ACG CCA CAA TAA GCA TCG CGA TTA CGG } \\
\text { GAT CCC ATT GGT AA-3' }\end{array}$ \\
\hline JS4 & 43mer & $\begin{array}{l}\text { 5'-CGG CAT CCT TGA TAT CCG CAG CAT CTT } \\
\text { TGT TTG TAT TAT ACT G-3' }\end{array}$ \\
\hline JS5 & $43 m e r$ & $\begin{array}{l}\text { 5'-CAG TAT AAT ACA AAC AAA GAT GCT GCG } \\
\text { GAT ATC AAG GAT GCC G-3' }\end{array}$ \\
\hline JS6 & 22mer & 5'-TGC TTA CAA TTT GGG ATG TTT C-3' \\
\hline JS9 & 20mer & 5'-TAA CGG TGC GCA CAT CAT CC-3' \\
\hline
\end{tabular}


Table 4: Primers used in this study, continued.

\begin{tabular}{|c|c|c|}
\hline Name & Size & Sequence \\
\hline JS10 & $43 \mathrm{mer}$ & $\begin{array}{l}\text { 5'-TTA CCA ATG GGA TCC CGT AAT CGT GAT } \\
\text { AAT TTG AGA AAT TGC G-3' }\end{array}$ \\
\hline JS11 & $43 \mathrm{mer}$ & $\begin{array}{l}\text { 5'-CGC AAT TTC TCA AAT TAT CAC GAT TAC } \\
\text { GGG ATC CCA TTG GTA A-3' }\end{array}$ \\
\hline JS12 & $47 \mathrm{mer}$ & $\begin{array}{l}\text { 5'-CAT TTT TTG ATT GGA AAA TGG TAT GCA } \\
\text { TCT TTG TTT GTA TTA TAC TG-3' }\end{array}$ \\
\hline JS13 & $47 \mathrm{mer}$ & $\begin{array}{l}\text { 5'-CAG TAT AAT ACA AAC AAA GAT GCA TAC } \\
\text { CAT TTT CCA ATC AAA AAA TG-3' }\end{array}$ \\
\hline JS14 & $20 \mathrm{mer}$ & 5'-AGG ACG AGT GGG AAG ATC TC-3' \\
\hline JS110 & $22 \mathrm{mer}$ & 5'-TTG CCA CTG CTC AAC CCT ACT C-3' \\
\hline JS111 & $22 \mathrm{mer}$ & 5'-GGG GAG ATT CAG GAC TTG TTC C-3' \\
\hline MC30 & $21 \mathrm{mer}$ & 5'-CAA GCG CGA CGG AGG GCT AGG-3' \\
\hline MC31 & $22 \mathrm{mer}$ & 5'-TCA CTC AAT ACG CGG CGG ACT C-3' \\
\hline MK30 & 39 mer & $\begin{array}{l}\text { 5'-GGT TGA CCA GCA CGA ACT CTA GCC GCC } \\
\text { ACG GAG AGC AAG-3' }\end{array}$ \\
\hline MK96 & $21 \mathrm{mer}$ & 5'-TCA TCG TAG AAC CCT ATC AAA-3' \\
\hline MK97 & $21 \mathrm{mer}$ & 5'-ATG GCG GAT GTT GAA ATG AAG-3' \\
\hline MK98 & $19 \mathrm{mer}$ & 5'-TTA CCG GCC GTA CTT CTG A-3' \\
\hline OLKM35 & 39 mer & $\begin{array}{l}\text { 5'-TAG GCC TGA GTG GCC TGT TGA AAC TAT } \\
\text { TTG GGA GTT ATC -3' }\end{array}$ \\
\hline OLKM36 & 35 mer & $\begin{array}{l}\text { 5'-TAG GCC TGA GTG GCC TGT TGA AAC TAT } \\
\text { TTG GGA GTT ATC-3' }\end{array}$ \\
\hline OLKM42 & $23 \mathrm{mer}$ & 5'-CAA TCA AGA ATG AAT TGA CTG AC-3' \\
\hline OLKM43 & $22 \mathrm{mer}$ & 5'-AGT TTA AGC CGG AAT AAA ACT C-3' \\
\hline OLKM67 & $21 \mathrm{mer}$ & 5'-CCA TAA CCC TAT TGC CAC TAG-3' \\
\hline OLKM68 & $24 \mathrm{mer}$ & 5'-GTA TGG GAT AGG AAA ATA ATA TAG-3' \\
\hline OLKM86 & $21 \mathrm{mer}$ & 5'-ATG GCC GAC AAG CAG AAG AAC-3' \\
\hline OLKM87 & 70mer & $\begin{array}{l}\text { 5'-GTG GTT CAT GAC CTT CTG TTT CAG GTC } \\
\text { GTT CGG GAT CTT GCA GGC CGG GCG CTT } \\
\text { GTA CAG CTC GTC CAT G-3' }\end{array}$ \\
\hline OLKM91 & $19 \mathrm{mer}$ & 5'-ATG GTG AGC AAG GGC GAG G-3' \\
\hline OZG192 & $27 \mathrm{mer}$ & $\begin{array}{l}\text { 5'-TCA GGG GCA GGG CAT GCT CAT GTA GAG- } \\
3^{\prime}\end{array}$ \\
\hline OZG207 & $24 \mathrm{mer}$ & 5'-GGT GGT AGC GGT GGT ATG GTG AGC-3' \\
\hline OZG209 & 43 mer & $\begin{array}{l}\text { 5'-GGT GGT AGC GGT GGT AAG AGA AGA TGG } \\
\text { AAA AAG AAT TTC ATA G-3' }\end{array}$ \\
\hline OZG504 & $20 \mathrm{mer}$ & 5'-CTA CTT GTA CAG TTC GTC CA-3' \\
\hline RH1 & 33 mer & $\begin{array}{l}\text { 5'-GCT GGG CCC TGT TGA AAC TAT TTG GGA } \\
\text { GTT ATC-3' }\end{array}$ \\
\hline RH2 & $38 \mathrm{mer}$ & $\begin{array}{l}\text { 5'-GTC GGG CCC GAT ATC CAT TTC ATA ATA } \\
\text { ATC ACG CAT TC-3' }\end{array}$ \\
\hline RH3 & $29 \mathrm{mer}$ & $\begin{array}{l}\text { 5'-CTT ATC GAT TCT GAT CCA TCT GCT CCC } \\
\text { AC-3' }\end{array}$ \\
\hline RH4 & $40 \mathrm{mer}$ & $\begin{array}{l}\text { 5'-CAA ATC GGC GGT GGT CTC TAA TCC ACC } \\
\text { AGA GTC CGG TTT C-3' }\end{array}$ \\
\hline RH5 & 40mer & $\begin{array}{l}\text { 5'-GAA ACC GGA CTC TGG TGG ATT AGA GAC } \\
\text { CAC CGC CGA TTT G-3' }\end{array}$ \\
\hline
\end{tabular}


Table 4: Primers used in this study, continued.

\begin{tabular}{|c|c|c|}
\hline Name & Size & Sequence \\
\hline RH6 & 39 mer & $\begin{array}{l}\text { 5'-ACG TAG AAT CTG GCC CAC ATT GTC TGA } \\
\text { GAG GAG GCA CTG-3' }\end{array}$ \\
\hline RH7 & $39 \mathrm{mer}$ & $\begin{array}{l}\text { 5'-CAG TCG CTC CTC TCA GAC AAT GTG GGC } \\
\text { CAG ATT CTA CGT-3' }\end{array}$ \\
\hline RH8 & $41 \mathrm{mer}$ & $\begin{array}{l}\text { 5'-GTG AAG AGC ATT GTT TGA GGC TCG CCA } \\
\text { CGG CAT TAG CAA TC-3' }\end{array}$ \\
\hline RH11 & $31 \mathrm{mer}$ & $\begin{array}{l}\text { 5'-GGC GAT ATC GTT GAT GTG TTC ATC ATC } \\
\text { TTC G-3' }\end{array}$ \\
\hline RH12 & $42 \mathrm{mer}$ & $\begin{array}{l}\text { 5'-TAC CAA TGG GAT CCC GTA ATG GTA GAG } \\
\text { ACA ATC TTC TAA GTA-3' }\end{array}$ \\
\hline RH13 & $39 \mathrm{mer}$ & $\begin{array}{l}\text { 5'-TAC TTA GAA GAT TGT CTC TAC CAT TAC } \\
\text { GGG ATC CCA TTG-3' }\end{array}$ \\
\hline RH14 & $42 \mathrm{mer}$ & $\begin{array}{l}\text { 5'-CGT CAA TTC TGT GAT TGC ACC GCA TCT } \\
\text { TTG TTT GTA TTA TAC-3' }\end{array}$ \\
\hline RH15 & $42 \mathrm{mer}$ & $\begin{array}{l}\text { 5'-GTA TAA TAC AAA CAA AGA TGC GGT GCA } \\
\text { ATC ACA GAA TTG ACG-3' }\end{array}$ \\
\hline RH16 & $31 \mathrm{mer}$ & $\begin{array}{l}\text { 5'-GAC GAT ATC GAT TGT CAT TGT CTT AGA } \\
\text { CAC C-3' }\end{array}$ \\
\hline RH17 & $19 \mathrm{mer}$ & 5'-ATG CCG CCA TAC ACT GCC G-3' \\
\hline RH18 & $20 \mathrm{mer}$ & 5'-TCA TTC CTC CAT AGC TCC CT-3' \\
\hline RH19 & $28 \mathrm{mer}$ & $\begin{array}{l}\text { 5'-GGC GAG CTC TCC GAC CAG GCC AGA AAG } \\
\text { G-3' }\end{array}$ \\
\hline RH20 & $41 \mathrm{mer}$ & $\begin{array}{l}\text { 5'-ACC CTC ATC GCA TTG CAA CCA TTA CGG } \\
\text { GAT CCC ATT GGT AA-3' }\end{array}$ \\
\hline RH21 & $41 \mathrm{mer}$ & $\begin{array}{l}\text { 5'-TTA CCA ATG GGA TCC CGT AAT GGT TGC } \\
\text { AAT GCG ATG AGG GT-3' }\end{array}$ \\
\hline RH22 & $43 \mathrm{mer}$ & $\begin{array}{l}\text { 5'-TCG TCA TTG AGT GGC TCA ACG CAT CTT } \\
\text { TGT TTG TAT TAT ACT G-3' }\end{array}$ \\
\hline RH23 & $43 \mathrm{mer}$ & $\begin{array}{l}\text { 5'-CAG TAT AAT ACA AAC AAA GAT GCG TTG } \\
\text { AGC CAC TCA ATG ACG A-3' }\end{array}$ \\
\hline RH24 & $28 \mathrm{mer}$ & $\begin{array}{l}\text { 5'-GAC GAG CTC CTG AGA GGA CCA GCC GAT } \\
\text { G-3' }\end{array}$ \\
\hline RH37 & $30 \mathrm{mer}$ & $\begin{array}{l}\text { 5'-TCC CAT ATG TTC AAT TGC TTC CTC AAT } \\
\text { CGC-3' }\end{array}$ \\
\hline RH38 & $29 \mathrm{mer}$ & $\begin{array}{l}\text { 5'-CGC ATC GAT GCC TCA AAC AAT GCT CTT } \\
\text { CA-3' }\end{array}$ \\
\hline RH43 & $31 \mathrm{mer}$ & $\begin{array}{l}\text { 5'-AAA ACG CGT AAG TTG TAT GCT TGT CTT } \\
\text { GGA G-3' }\end{array}$ \\
\hline RH44 & $42 \mathrm{mer}$ & $\begin{array}{l}\text { 5'-CAG TGC CTC CTC TCA GAC ATA TGT AGA } \\
\text { CAG ATT TTG AGC TTG-3' }\end{array}$ \\
\hline RH45 & $42 \mathrm{mer}$ & $\begin{array}{l}\text { 5'-CAA GCT CAA AAT CTG TCT ACA TAT GTC } \\
\text { TGA GAG GAG GCA CTG-3' }\end{array}$ \\
\hline RH46 & $41 \mathrm{mer}$ & $\begin{array}{l}\text { 5'-CCC ATT CAT AAG AAG ACA CGT GCC TCA } \\
\text { AAC AAT GCT CTT CA-3' }\end{array}$ \\
\hline RH47 & $41 \mathrm{mer}$ & $\begin{array}{l}\text { 5'-TGA AGA GCA TTG TTT GAG GCA CGT GTC } \\
\text { TTC TTA TGA ATG GG-3' }\end{array}$ \\
\hline
\end{tabular}


Table 4: Primers used in this study, continued.

\begin{tabular}{|c|c|c|}
\hline Name & Size & Sequence \\
\hline RH48 & $31 \mathrm{mer}$ & $\begin{array}{l}\text { 5'-TTT ACG CGT CTA CTG CTT AAT GAT TGG } \\
\text { TAA G-3' }\end{array}$ \\
\hline RH78 & $21 \mathrm{mer}$ & 5'-TGC GCT TCA ATT AGA ATG GTC-3' \\
\hline RH79 & 39 mer & $\begin{array}{l}\text { 5'-GAC CCA ACA ACC ATG ATA CCA GGC GTG } \\
\text { CGA TGG TGC GAG-3' }\end{array}$ \\
\hline RH80 & $39 \mathrm{mer}$ & $\begin{array}{l}\text { 5'-CTC GCA CCA TCG CAC GCC TGG TAT CAT } \\
\text { GGT TGT TGG GTC-3' }\end{array}$ \\
\hline RH81 & $42 \mathrm{mer}$ & $\begin{array}{l}\text { 5'-TGT AGT CGG GAA GAT TAA ATG TAG CAT } \\
\text { CCA CAT GAT CGA CAG-3' }\end{array}$ \\
\hline RH82 & $42 \mathrm{mer}$ & $\begin{array}{l}\text { 5'-CTG TCG ATC ATG TGG ATG CTA CAT TTA } \\
\text { ATC TTC CCG ACT ACA-3' }\end{array}$ \\
\hline RH83 & $18 \mathrm{mer}$ & 5'-GGC AGG AGA CGA CGG ACA-3' \\
\hline RH84 & $20 \mathrm{mer}$ & 5'-TCC TCT CCA TCT ACG AAT GC-3' \\
\hline RH87 & $42 \mathrm{mer}$ & $\begin{array}{l}\text { 5'-CAA CAC ATC CCT TCA GAA CAC AGA TGG } \\
\text { CCT CTT GCA TCT TTG-3' }\end{array}$ \\
\hline RH88 & $42 \mathrm{mer}$ & $\begin{array}{l}\text { 5'-CAA AGA TGC AAG AGG CCA TCT GTG TTC } \\
\text { TGA AGG GAT GTG TTG-3' }\end{array}$ \\
\hline RH89 & $20 \mathrm{mer}$ & 5'-TTG GGC ACG ATT TAG TGT CG-3' \\
\hline RH95 & $39 \mathrm{mer}$ & $\begin{array}{l}\text { 5'-ACG CCG ACC ATT GCG CCA AGG CCA ATT } \\
\text { GAT TAC GGG ATC-3' }\end{array}$ \\
\hline RH96 & 39 mer & $\begin{array}{l}\text { 5'-GAT CCC GTA ATC AAT TGG CCT TGG CGC } \\
\text { AAT GGT CGG CGT-3' }\end{array}$ \\
\hline RH97 & $20 \mathrm{mer}$ & 5'-TCA TTC CTC CAT AGC TCC CT-3' \\
\hline RH98 & $38 \mathrm{mer}$ & $\begin{array}{l}\text { 5'-GGC GGC AGT GTA TGG CGG CGT GGC GAT } \\
\text { GGA GCG CAT GA-3' }\end{array}$ \\
\hline RH99 & $55 \mathrm{mer}$ & $\begin{array}{l}\text { 5'-GGC GGC AGT GTA TGG CGG CGT GGC GAT } \\
\text { GGA GCG CAT GAT ATA GAC GTT GTG GCT G-3' }\end{array}$ \\
\hline RH100 & $40 \mathrm{mer}$ & $\begin{array}{l}\text { 5'-ACA GAA GGT CAT GAA CCA CGC GGA TGT } \\
\text { TGA AAT GAA GGA G-3' }\end{array}$ \\
\hline RH101 & $41 \mathrm{mer}$ & $\begin{array}{l}\text { 5'-ACA GAA GGT CAT GAA CCA CTT GAT CAA } \\
\text { GGT CCG TAC ACT TA-3' }\end{array}$ \\
\hline RH102 & $43 \mathrm{mer}$ & $\begin{array}{l}\text { 5'-ACA GAA GGT CAT GAA CCA CCT AAA AAT } \\
\text { CTG GTC TAT GAA ACA A-3' }\end{array}$ \\
\hline RH201 & 20mer & 5'-AGC CAG GCA TAA CTC GTA GT-3' \\
\hline RH202 & 43 mer & $\begin{array}{l}\text { 5'-TTA CCA ATG GGA TCC CGT AAT ATT TCT } \\
\text { TGA CTC AAG AAA CAG C-3' }\end{array}$ \\
\hline RH203 & $43 \mathrm{mer}$ & $\begin{array}{l}\text { 5'-GCT GTT TCT TGA GTC AAG AAA TAT TAC } \\
\text { GGG ATC CCA TTG GTA A-3' }\end{array}$ \\
\hline RH204 & $44 \mathrm{mer}$ & $\begin{array}{l}\text { 5'-GGC TTG TAG CCA TAT CTC ATT GCA TCT } \\
\text { TTG TTT GTA TTA TAC TG-3' }\end{array}$ \\
\hline RH205 & $44 \mathrm{mer}$ & $\begin{array}{l}\text { 5'-CAG TAT AAT ACA AAC AAA GAT GCA ATG } \\
\text { AGA TAT GGC TAC AAG CC-3' }\end{array}$ \\
\hline RH206 & $24 \mathrm{mer}$ & 5'-AAT AAG AAG ATA TAC TTC AAG TCA-3' \\
\hline RH212 & $19 \mathrm{mer}$ & 5'-TCT TAA GCC GGC GCT CAC T-3' \\
\hline RH214b & $19 \mathrm{mer}$ & 5'-ATG TCG CGC TCT TCC GCA G-3' \\
\hline RH215 & $19 \mathrm{mer}$ & 5'-CGA CCG CCT AGC TTT TCC T-3' \\
\hline RH221 & $20 \mathrm{mer}$ & 5'-TGG AGG ACT TTA CGA GTC GC-3' \\
\hline
\end{tabular}


Table 4: Primers used in this study, continued.

\begin{tabular}{|c|c|c|}
\hline Name & Size & Sequence \\
\hline RH222 & $52 \mathrm{mer}$ & $\begin{array}{l}\text { 5'-GCC CTT GCT CAC CAT ACC ACC GCT ACC } \\
\text { ACC ATT CAG AAA GCC TTT ACA TCC G-3' }\end{array}$ \\
\hline RH223 & $49 \mathrm{mer}$ & $\begin{array}{l}\text { 5'-CTC TAC ATG AGC ATG CCC TGC CCC TGA } \\
\text { ATT TCT TGA CTC AAG AAA CAG C-3' }\end{array}$ \\
\hline RH224 & $22 \mathrm{mer}$ & 5'-ACT GCT TAT GAT GAC ACA GTT G-3' \\
\hline RH227 & $55 \mathrm{mer}$ & $\begin{array}{l}\text { 5'-CTT TTT CCA TCT TCT CTT ACC ACC GCT } \\
\text { ACC ACC ATT CAG AAA GCC TTT ACA TCC G-3' }\end{array}$ \\
\hline RH234 & $19 \mathrm{mer}$ & 5'-ACG AGA CTC TCG CAT ACC G-3' \\
\hline RH235 & $18 \mathrm{mer}$ & 5'-ACG GGT GAT GTC CTC CGC-3' \\
\hline RH248 & $22 \mathrm{mer}$ & 5'-CAT TAA TAA TTC CAA GCA CAG C-3' \\
\hline RH249 & $41 \mathrm{mer}$ & $\begin{array}{l}\text { 5'-GAG CAT TGT TTG AGG CGA ATT TAA CGC } \\
\text { ACG CAT ATG CGC AG-3' }\end{array}$ \\
\hline RH250 & $41 \mathrm{mer}$ & $\begin{array}{l}\text { 5'-CTG CGC ATA TGC GTG CGT TAA ATT CGC } \\
\text { CTC AAA CAA TGC TC-3' }\end{array}$ \\
\hline RH251 & $39 \mathrm{mer}$ & $\begin{array}{l}\text { '-TCG TTC ATG CGC ATT GCG CAC TGT CTG } \\
\text { AGA GGA GGC ACT-3' }\end{array}$ \\
\hline RH252 & $39 \mathrm{mer}$ & $\begin{array}{l}\text { 5'-AGT GCC TCC TCT CAG ACA GTG CGC AAT } \\
\text { GCG CAT GAA CGA-3' }\end{array}$ \\
\hline RH253 & $21 \mathrm{mer}$ & 5'-GGA TTA ATT CGA CGC ACC ATA-3' \\
\hline RH254 & $20 \mathrm{mer}$ & 5'-ACA GTA CTG TAG GGT GGC TG-3' \\
\hline RH255 & $36 \mathrm{mer}$ & $\begin{array}{l}\text { 5'-TAC CAA TGG GAT CCC GTA ATG GTG AAG } \\
\text { TAT TCT TGT-3' }\end{array}$ \\
\hline RH256 & $41 \mathrm{mer}$ & $\begin{array}{l}\text { 5'-ACG CAC AAG AAT ACT TCA CCA TTA CGG } \\
\text { GAT CCC ATT GGT AA-3' }\end{array}$ \\
\hline RH257 & $44 \mathrm{mer}$ & $\begin{array}{l}\text { 5'-GAA CCG AAA TAT ACG TTA ACC GCA TCT } \\
\text { TTG TTT GTA TTA TAC TG-3' }\end{array}$ \\
\hline RH258 & $42 \mathrm{mer}$ & $\begin{array}{l}\text { 5'-TAT AAT ACA AAC AAA GAT GCG GTT AAC } \\
\text { GTA TAT TTC GGT TCG-3' }\end{array}$ \\
\hline RH259 & $19 \mathrm{mer}$ & 5'-GTC AGT ACT GGT TGC GCC G-3' \\
\hline RH260 & $19 \mathrm{mer}$ & 5'-CCA GGC CTA GGT AGT GCA T-3' \\
\hline RH261 & $39 \mathrm{mer}$ & $\begin{array}{l}\text { 5'-TAC CAA TGG GAT CCC GTA ATT AGA ACA } \\
\text { ATG CCG CCG CGG-3' }\end{array}$ \\
\hline RH262 & 33 mer & $\begin{array}{l}\text { 5'-CCG CGG CGG CAT TGT TCT AAT TAC GGG } \\
\text { ATC CCA-3' }\end{array}$ \\
\hline RH263 & $42 \mathrm{mer}$ & $\begin{array}{l}\text { 5'-TGT CTA TAG AAT TTG GTT CAG CAT CTT } \\
\text { TGT TTG TAT TAT ACT-3' }\end{array}$ \\
\hline RH264 & $43 \mathrm{mer}$ & $\begin{array}{l}\text { 5'-CAG TAT AAT ACA AAC AAA GAT GCT GAA } \\
\text { CCA AAT TCT ATA GAC A-3' }\end{array}$ \\
\hline RH265 & $20 \mathrm{mer}$ & 5'-ATA CGA TGC GCT TGT CAA CC-3' \\
\hline RH316 & $21 \mathrm{mer}$ & 5'-TGG TAT CAT GGT TGT TGG GTC-3' \\
\hline RH317 & $20 \mathrm{mer}$ & 5'-AGC ATC CAC ATG ATC GAC AG-3' \\
\hline RH318 & $39 \mathrm{mer}$ & $\begin{array}{l}\text { 5'-CTG TCG ATC ATG TGG ATG CTA TAC CGG } \\
\text { GCG CAA TGG AGC-3' }\end{array}$ \\
\hline RH319 & $43 \mathrm{mer}$ & $\begin{array}{l}\text { 5'-GAC CCA ACA ACC ATG ATA CCA TAT GAA } \\
\text { ACC ACA GTC TTG GTA A-3' }\end{array}$ \\
\hline RH320 & $43 \mathrm{mer}$ & $\begin{array}{l}\text { 5'-CTG TCG ATC ATG TGG ATG CTT GAT GCA } \\
\text { AAT ATG TAT TAG TTC G-3' }\end{array}$ \\
\hline RH360b & $19 \mathrm{mer}$ & 5'-ACT CGC TTT CTG CAC TGG C-3' \\
\hline
\end{tabular}


Table 4: Primers used in this study, continued.

\begin{tabular}{|l|l|l|}
\hline \multicolumn{1}{|c|}{ Name } & \multicolumn{1}{c|}{ Size } & \multicolumn{1}{c|}{ Sequence } \\
\hline RH361b & 20mer & 5'-TAC TCC ATA TCT ACG CCG TG-3' \\
\hline RH395 & $24 m e r$ & 5'-AGT ACT AAT CGG ATC ATG GGT ACG-3' \\
\hline RH396 & $24 m e r$ & 5'-AGT ACT TCT TTC CTC GGT ATT CGA-3' \\
\hline RH397 & $26 m e r$ & 5'-AGG CCT ACG GAC GGT ATA CGG AGT AG-3' \\
\hline RT-MC1 & $22 \mathrm{mer}$ & 5'-GAC GAT TCA CCA ACC CAA GAG A-3' \\
\hline RT-MC2 & $22 \mathrm{mer}$ & 5'-CTA CCT TCC AGC CAC CCA AAC T-3' \\
\hline UW45 & 24mer & 5'-TCG CGA CCG CCT TTG TAG ATA ATG-3' \\
\hline
\end{tabular}

\subsubsection{Aspergillus nidulans strains used in this study}

Aspergillus nidulans strains with their respective genotypes used in this study are listed in table 5. Details on strain construction are given below.

Table 5: A. nidulans strains used in this study.

\begin{tabular}{|c|c|c|}
\hline Name & Genotype & Reference \\
\hline A4 & Glasgow wild type & FGSC \\
\hline TNO2A3 & pyro $A 4$, pyrG89, $\triangle n k u A: \because \arg B ;$ veAl & (Nayak et al., 2006) \\
\hline AGB152 & pyroA4, pyrG89 & (Busch et al., 2003) \\
\hline AGB209 & pyroA4, pyrG89, $\triangle \operatorname{csn} E::$ pyr-4 & (Busch et al., 2003) \\
\hline AGB350 & $\begin{array}{l}\text { pyroA4, pyrG89, } 4 \text { sumO::ptrA; } \\
\text { sumO(p)::sumO::sumO(t)/nat }\end{array}$ & (Laubinger, 2008) \\
\hline AGB380 & 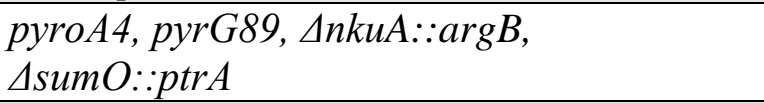 & (Laubinger, 2008) \\
\hline AGB445 & $\begin{array}{l}\text { pyro } A 4, \text { pyrG89, } \triangle n k u A:: \arg B \text {; } \\
\text { histone::mrfp }\end{array}$ & (Bayram et al., 2009) \\
\hline AGB500 & $\begin{array}{l}\text { pyroA4, pyrG89, } \\
\text { niiA }(p):: \text { neyfp }:: d c n A:: n i i A(t) \\
\text { niaD }(p):: \text { ceyfp::niaD }(t), \text { pyrG }\end{array}$ & $\begin{array}{l}\text { (von Zeska Kress et } \\
\text { al., 2012) }\end{array}$ \\
\hline AGB501 & $\begin{array}{l}\text { pyroA4, pyrG89, } \\
\text { niiA }(p):: \text { neyfp::dcnA::niiA }(t) ; \operatorname{niaD}(p):: \\
\text { neddH::ceyfp::niaD }(t) / \text { pyrG }\end{array}$ & $\begin{array}{l}\text { (von Zeska Kress et } \\
\text { al., 2012) }\end{array}$ \\
\hline AGB502 & $\begin{array}{l}\text { pyroA4, pyrG89, } \\
\text { niiA }(p):: \text { neyfp }:: d c n A: \because n i i A(t) ; \\
\text { niaD }(p):: \operatorname{rbxA}:: \text { ceyfp::niaD }(t) / \text { pyrG }\end{array}$ & $\begin{array}{l}\text { (von Zeska Kress et } \\
\text { al., 2012) }\end{array}$ \\
\hline AGB503 & $\begin{array}{l}\text { pyroA4, pyrG89, } \\
\text { niiA }(p):: \text { neyfp::dcnA::niiA }(t) ; \\
\text { niaD }(p):: \text { ubcL::ceyfp::niaD }(t) / p y r G\end{array}$ & $\begin{array}{l}\text { (von Zeska Kress et } \\
\text { al., 2012) }\end{array}$ \\
\hline AGB504 & pyroA4, pyrG89, $\triangle n k u A: \because \operatorname{argB}, \triangle d c n A:: p t r A$ & $\begin{array}{l}\text { (von Zeska Kress et } \\
\text { al., 2012) }\end{array}$ \\
\hline AGB551 & pyroA4, pyrG89, $\triangle n k u A: \because \arg B, v e A^{+}$ & (Bayram et al., 2012) \\
\hline AGB678 & pyroA4, pyrG89, gpdA(p)::fbx15::sgfp, ble & $\begin{array}{l}\text { (von Zeska Kress et } \\
\text { al., 2012) }\end{array}$ \\
\hline AGB679 & $\begin{array}{l}\text { pyroA4, pyrG89, } \Delta c s n E:: p y r-4, \\
\text { gpdA(p)::fbx15::sgfp, bleo }\end{array}$ & $\begin{array}{l}\text { (von Zeska Kress et } \\
\text { al., 2012) }\end{array}$ \\
\hline
\end{tabular}


Table 5: A. nidulans strains used in this study, continued.

\begin{tabular}{|c|c|c|}
\hline Name & Genotype & Reference \\
\hline AGB688 & $\begin{array}{l}\text { pyroA4, pyrG89, } \triangle \text { nkuA::argB, } \\
\operatorname{sumO}(p):: \operatorname{tap}: \because \operatorname{sumO}: \because \operatorname{sum} O(t):: \text { pyrG }\end{array}$ & This study \\
\hline AGB689 & $\begin{array}{l}\text { pyro } A 4, \text { pyrG89, } \triangle n k u A:: \arg B \\
\text { AulpA::pyroA }\end{array}$ & This study \\
\hline AGB690 & pyroA4, pyrG89, $\triangle n k u A:: \arg B ; \triangle u b c N:: p \operatorname{tr} A$ & This study \\
\hline AGB691 & pyroA4, pyrG89, $\triangle n k u A: \because \arg B ; \triangle \operatorname{sizA}::$ ptrA & This study \\
\hline AGB692 & pyroA4, pyrG89, $\triangle n k u A: \because \arg B ; \triangle \operatorname{aos} A: \because p \operatorname{pt} A$ & This study \\
\hline AGB693 & $\begin{array}{l}\text { pyroA4, pyrG89, } \triangle n k u A:: \arg B \\
\text { AubaB::pyrG }\end{array}$ & This study \\
\hline AGB694 & $\begin{array}{l}\text { pyroA4, pyrG89, } \triangle n k u A: \because \arg B ; \triangle \operatorname{aos} A: \because p t r A, \\
\text { AubaB::pyrG }\end{array}$ & This study \\
\hline AGB695 & 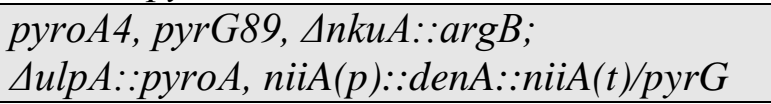 & This study \\
\hline AGB696 & $\begin{array}{l}\text { pyroA4, pyrG89, } \\
\text { niiA(p):::denA::niiA(t)/pyrG }\end{array}$ & This study \\
\hline AGB704 & $\begin{array}{l}\text { pyro } A 4, \text { pyrG89, } \triangle \text { nkuA::argB; } \\
\text { \aosA::ptrA/aosA::pyroA }\end{array}$ & This study \\
\hline AGB705 & $\begin{array}{l}\text { pyroA4, pyrG89, } \triangle \text { nkuA::argB; } \\
\text { AubaB::pyrG/ubaB::pyroA }\end{array}$ & This study \\
\hline AGB706 & $\begin{array}{l}\text { pyroA4, pyrG89, } \triangle \text { nkuA::argB; } \\
\text { AubcN::ptrA/ubcN::pyroA }\end{array}$ & This study \\
\hline AGB707 & 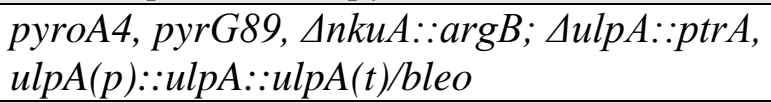 & This study \\
\hline AGB712 & pyroA4, pyrG89, $\triangle n k u A:: \arg B ; \triangle$ setA $:: p \operatorname{pt} A$ & This study \\
\hline AGB713 & $\begin{array}{l}\text { pyroA4, pyrG89, } \triangle \text { nkuA::argB; } \\
\text { setA }(\text { p)::setA::setA }(t) / \text { bleo }\end{array}$ & This study \\
\hline AGB714 & $\begin{array}{l}\text { pyro } A 4, \text { pyrG89, } \triangle n k u A: \because \arg B ; \\
\text { set } A(p) \therefore \text { setA }: \because \text { set } A(t) / b l e o, \Delta \text { set } A: \therefore \text { ptrA }\end{array}$ & This study \\
\hline AGB715 & $\begin{array}{l}\text { pyroA4, pyrG89, } \triangle n k u A: \because \arg B \\
\text { histone::mrfp; } \text {,setA::ptrA }\end{array}$ & This study \\
\hline AGB716 & $\begin{array}{l}\text { pyroA4, pyrG89, } \\
\text { niiA(p)::gfp::setA::niiA(t)/pyrG }\end{array}$ & This study \\
\hline AGB717 & pyroA4, pyrG89, AnkuA::argB; $\Delta$ ulpB::ptrA & This study \\
\hline AGB733 & $\begin{array}{l}\text { pyroA4, pyrG89, } \triangle \text { nkuA::argB; } \\
\text { ulpB }(p):: \text { ulpB::ulpB(t)/bleo }\end{array}$ & This study \\
\hline AGB734 & $\begin{array}{l}\text { pyroA4, pyrG89, } \triangle \text { nkuA::argB; } \\
\text { ulpB }(\text { p)::ulpB::ulpB(t)/bleo; } \Delta \text { ulpB::ptrA }\end{array}$ & This study \\
\hline AGB735 & $\begin{array}{l}\text { pyroA4, pyrG89, } \triangle \text { nkuA::argB; } \\
\triangle A N 1192:: p y r G\end{array}$ & This study \\
\hline AGB736 & $\begin{array}{l}\text { pyroA4, pyrG89, } \triangle \text { nkuA::argB, } \\
\operatorname{set} A(p):: \text { setA::tap::gpdA }(p):: \text { nat::3'region } \\
\text { setA }\end{array}$ & This study \\
\hline AGB737 & 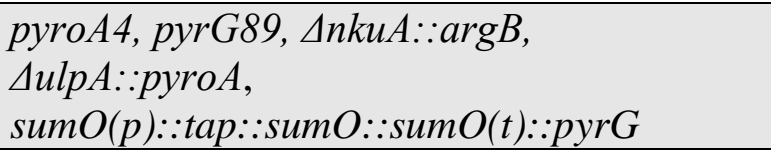 & This study \\
\hline AGB738 & $\begin{array}{l}\text { pyroA4, pyrG89, } \triangle n k u A: \because \arg B \\
\triangle A N 4149:: \text { ptrA }\end{array}$ & This study \\
\hline
\end{tabular}


Table 5: A. nidulans strains used in this study, continued.

\begin{tabular}{|c|l|l|}
\hline Name & \multicolumn{1}{|c|}{ Genotype } & Reference \\
\hline AGB739 & $\begin{array}{l}\text { pyroA4, pyrG89, } \triangle n k u A: \because \arg B, \\
\Delta A N 4491:: p t r A\end{array}$ & This study \\
\hline
\end{tabular}

FGSC: Fungal Genetic Stock Center (University of Kansas Medical Center, Kansas City, KS, USA)(McCluskey et al., 2010), t: terminator, p: promoter

\subsubsection{Plasmid and strain constructions}

\subsection{Plasmid and strain construction for the TAP::SumO strains}

For expression of the TAP::SumO fusion protein the 5' flanking region of A. nidulans sumO was amplified with the ApaI restriction site containing primers RH1 and RH2. The PCR product was ligated into the ApaI restriction site of pME2968. The 3' flanking region of sumO was amplified with the primers RH8 and RH37 that contain NdeI restriction sites. The amplicon was ligated into the NdeI site of the plasmid, carrying the 5' flanking region, resulting in pME3967. sumO cDNA was amplified with RH3 and RH5 from A. nidulans cDNA. The sumO terminator was amplified with RH4 and RH7 from genomic DNA and the A. fumigatus pyrG marker with RH6 and RH38 from pME3160. The three fragments were fused by fusion PCR (Szewczyk et al., 2006) with RH3 and RH38. The cassette was ligated into the ClaI restriction site of pME3967 resulting in pME3968. The whole cassette was restricted from the plasmid with $\mathrm{NruI}$ and $P s c \mathrm{I}$ and transformed into the strains TNO2A3 and AGB689. Correct replacement of the sumO locus by tap::sumO was confirmed by Southern hybridization using a sumO probe amplified with OLKM35 and OLKM42 and restriction with ScaI or XhoI. The expression of the fusion protein was confirmed by immunoblotting with a calmodulin binding protein antibody. The strains were named AGB688 and AGB737 (Harting et al., 2013).

With the cassette used to express tap::sumO the locus of the neighbouring gene AN1192 is disturbed. To exclude an influence on the strain, a control strain carrying only the pyrG marker surrounded by the $\operatorname{sum} O$ terminator and the 3' flanking region but not the tap::sumO cassette was constructed. The fragment was amplified with RH4 and RH37 from pME3968 and transformed into TNO2A3 resulting in AGB735. Insertion of the cassette was verified in Southern hybridisation using a sumO terminator probe amplified with OLKM36 and OLKM43 and HindIII or $B g l \mathrm{I}$ restriction. Phenotypical analyses of the AN1192 deletion strain revealed 
neither an impact on asexual nor on sexual development suggesting no global impact on development and protein expression.

\subsection{PCR-mediated construction of deletion cassettes and deletion strain construction for $u l p A, u l p B, a o s A, u b a B, u b c N$ and $\operatorname{siz} A$}

The deletion cassettes for $u l p A, u l p B$, aosA, ubaB, ubcN and sizA were constructed using fusion PCR (Szewczyk et al., 2006). The coding region of the genes $u l p A, u l p B$ and $u b c N$ as well as the whole genes (including UTRs) $a o s A, u b a B$ and sizA were replaced by marker cassettes. The 5' and 3' flanking regions of the respective genes were amplified from A. nidulans genomic DNA with the following primers: $u l p A-\mathrm{RH} 78$ and RH79, RH82 and RH83; ulpB-RH84 and RH96, RH88 and RH89; aosA-RH254 and RH255, RH258 and RH259; $u b a B-\mathrm{RH} 248$ and RH249, RH252 and RH253; $u b c N-$ RH19 and RH21, RH23 and RH24; sizA-RH260 and RH261, RH264 and RH265. For several knockout cassettes the ptrA marker was used: $u l p B$ (amplified with RH95 and RH87), aosA (amplified with RH256 and RH257), $u b c N$ (amplified with RH20 and RH22) and sizA (amplified with RH262 and RH263). For the deletion cassette of $u l p A$ the pyroA marker amplified with RH80 and RH81 and for the $u b a B$ construct the $p y r G$ marker amplified with RH250 and RH251 was used. The corresponding fusions were generated with the following primer pairs: $u l p A-\mathrm{RH} 78$ and RH83; aosA-RH254 and RH259; ubaB-RH248 and RH253; $u b c N-$ RH19 and RH24; sizA-RH260 and RH265. All cassettes were subcloned into the pJET1.2/blunt vector resulting in plasmids pME3979, pME3977, pME3976, pME3984 and pME3978, respectively. For A. nidulans transformations the deletion cassettes were restricted from the plasmids using the following enzymes: pME3979 with AseI, pME3977 with ScaI, pME3976 with AseI, pME3984 with HindIII, and pME3978 with AvrII. The deletion cassettes were transformed into TNO2A3 resulting in AGB689-AGB693 and AGB717. The double deletion strain $\triangle a o s A / \triangle u b a B$ (AGB694) was obtained by transforming AGB692 with the $u b a B$ deletion cassette restricted from pME3976 (Harting et al., 2013). All deletion strains were confirmed by Southern hybridizations using the following enzymes and probes: AGB689-restriction with NcoI or AseI, probe 5' flanking region; AGB690-restriction with $K p n \mathrm{I}$, probe 3 ' flanking region or restriction with $E c o R \mathrm{~V}$, probe 5' flanking region; AGB691-restriction with $\mathrm{Nco}$ I or XhoI, probe 5' flanking region or restriction with $\mathrm{NcoI}$ probe 3' flanking region; AGB692-restriction with $B g l \mathrm{I}$, probe 3' flanking 
region; AGB693-restriction with HindIII, probe 5' flanking region or restriction EcoRV or XhoI, probe 3' flanking region; AGB717-BamHI, BglI or NcoI, probe 3' flanking region or restriction with HincII or NcoI, probe 3' flanking region.

\subsection{Plasmid and strain construction for complementation of the $u l p A$ deletion strain}

In order to complement the $u l p A$ deletion strain, the gene including $5^{\prime}$ and $3^{\prime}$ flanking regions was amplified from genomic DNA with RH234 and RH235. The amplicon was cloned into the EcoRV restriction site of pME3281 resulting in pME3985. The plasmid was transformed into AGB689 resulting in AGB707 (Harting et al., 2013). Ectopical integration was confirmed by Southern hybridization using restriction with AseI and the 5' flanking region as probe.

\subsection{Plasmid and strain construction for complementation of the $u l p B$ deletion strain}

A plasmid for ectopical reintroduction of the $u l p B$ locus was constructed. The $u l p B$ gene including promoter and terminator region was amplified with UW45 and RH89 and ligated into the EcoRV restriction site of pME3281 resulting in pME4067. Due to the sensitive nature of the $u l p B$ deletion strain, the following strategy was applied: for complementation of the $u l p B$ deletion strain, pME4067 was ectopically integrated in TNO2A3 and ectopical integration as well as the integrity of the original locus was confirmed with Southern hybridization using restriction with HincII or NcoI and the 5' flanking region as probe. The strain was named AGB733. Next, the $u l p B$ deletion cassette was restricted from pME4064 and introduced in AGB733 resulting in AGB734 (Harting et al., 2013). The replacement of the endogenous locus and the maintenance of the ectopical complementation construct was confirmed by Southern hybridization as described before.

\subsection{Plasmid and strain construction for complementation of the aos $A$ deletion strain}

To complement the aosA deletion strain, the gene was reintroduced at the original locus. The 5' flanking region together with the gene and the terminator 
region was amplified with $\mathrm{RH} 360 \mathrm{~b}$ and $\mathrm{RH} 319$. The A. fumigatus pyroA marker was amplified from pME3979 with RH316 and RH317 and the 3' flanking region was amplified with RH320 and RH361b. The three fragments were fused with RH360b and RH361b and cloned into the pJET1.2/blunt vector resulting in pME3994. The cassette was amplified from the plasmid with RH360b and RH361b and transformed into AGB692 resulting in AGB704 (Harting et al., 2013). Integration of the cassette was confirmed by Southern hybridization using SacI restriction and the 3' flanking region as probe.

\subsection{Plasmid and strain construction for complementation of the $u b a B$ deletion strain}

For reintroduction of the $u b a B$ gene into the original locus of the deletion strain, the 5' flanking region together with the gene and the terminator region was amplified from genomic DNA with RH395 and RH397. The PCR fragment was cloned into the pJET1.2/blunt vector resulting in pME3986. The A. fumigatus pyroA marker was amplified with RH316 and RH317 and the 3' flanking region with RH318 and RH396 from genomic DNA. The two fragments were fused by fusion PCR (Szewczyk et al., 2006) with RH316 and RH396. pME3986 was restricted with StuI and the fusion product was ligated with the vector resulting in pME3987. The cassette from the vector was transformed into AGB693 resulting in AGB705 (Harting et al., 2013). Homologous integration at the $u b a B$ locus was confirmed by Southern hybridization using EcoRV or XhoI restriction and the 3' flanking region as probe.

\subsection{Plasmid and strain construction for complementation of the $u b c N$ deletion strain}

To complement the $u b c N$ deletion strain, the gene was reintroduced at the original locus. For construction of the cassette, the gene together with 5' and 3' flanking region was amplified with $\mathrm{RH} 19$ and $\mathrm{RH} 24$. The amplicon was ligated into the pJET1.2/blunt vector. Then the plasmid was linearized with EcoRV restricting in the 3 ' flanking region behind the terminator. The A. fumigatus pyroA marker was amplified with RH80 and RH81 and ligated with the plasmid resulting in pME3995. The cassette from the plasmid was transformed into AGB690 resulting in AGB706 
(Harting et al., 2013). Correct integration at the locus was confirmed by Southern hybridization using KpnI restriction and the 3' flanking region as probe.

\subsection{Plasmid and strain construction for $\operatorname{den} A$ overexpression}

For ectopical overexpression of $\operatorname{den} A$, the gene was amplified from genomic DNA with MC30 and MC31. The PCR fragment was ligated into the SwaI restriction site of pME3160. The resulting plasmid, pME4068, was transformed into AGB152 and AGB689 resulting in AGB695 and AGB696, respectively (Harting et al., 2013). Integration of the plasmid was confirmed by Southern hybridization using a probe (amplified with OLKM67 and OLKM68) annealing in the nitrate promoter region and restriction with HindIII. Deletion of $u l p A$ was confirmed as described above. The strong expression of $\operatorname{den} A$ was monitored by qRT-PCR with the primers RT-MC1 and RT-MC2.

\subsection{Plasmid and strain construction for setA deletion}

In order to delete setA, a fusion cassette of the 5' flanking region of the gene (amplified with RH201 and RH202), the ptrA marker (amplified with RH203 and RH204 from pME3024) and the gene's 3' flanking region (amplified with RH205 and RH206) was used. The PCR products were fused using the oligonucleotides RH201 and RH206. The amplicon was subcloned into the pJET1.2/blunt vector resulting in pME3996. For A. nidulans transformation in AGB551, the cassette was excised from the plasmid. The resulting setA deletion strain was named AGB712 (Harting et al., 2013). The correct replacement of the gene was confirmed by Southern hybridization with $E c o R V, E c o R I$ or $N c o$ I restriction and the 3' flanking region as probe as well as restriction with $\mathrm{XhoI}$ and the $5^{\prime}$ flanking region as probe.

\subsection{Complementation of the setA deletion strain}

For ectopical reintroduction a plasmid was constructed carrying the gene with $500 \mathrm{bp}$ of the $5^{\prime}$ and the 3 ' flanking region. This fragment was amplified from genomic DNA of a wild type strain with the primers RH212 and RH215. The amplicon was ligated into the EcoRV restriction site of pME3281 resulting in pME3997. Due to the very sensitive nature of the deletion strain, the cassette was first ectopically introduced into the wild type strain AGB551 resulting in AGB713. 
The integration as well as the integrity of the setA locus was confirmed by Southern hybridization with $N c o$ I, $B g l \mathrm{I}$ or $B a m H I$ restriction and the 5' flanking region as probe. Next, the setA deletion cassette from pME3996 was used to replace the setA gene. Homologous integration of the construct at the setA locus as well as the presence of the ectopically integrated complementation construct was confirmed applying Southern hybridizations as described before. The strain was named AGB714 (Harting et al., 2013).

\subsection{Plasmid and strain construction for SetA::TAP}

For expression of a TAP-tagged setA version the wild type setA locus was replaced by a setA::tap gene fusion. The 5' flanking region together with the setA gene was amplified with the oligonucleotides RH206 and RH227. A fusion construct of the tap cassette and a $g p d A$ promoter driven nourseothricin resistance marker was amplified from pME3928 with the primers OZG209 and OZG192. The 3' flanking region of the gene was amplified using RH223 and RH201. All three amplicons were fused by fusion PCR (Szewczyk et al., 2006) with the primers RH221 and RH224. The construct was subcloned into the pJET1.2/blunt vector resulting in pME4066. The cassette was excised from the plasmid and used to transform AGB551 resulting in AGB736 (Harting et al., 2013). Homologous recombination at the setA locus was verified by Southern hybridization using BglI or HindIII restriction and the 3' flanking region as probe.

\subsection{Plasmid and strain construction for expression of histone::mrfp and SetA overexpression}

For the expression of histone::mrfp in the setA deletion background, the setA deletion cassette from pME3996 was transformed into AGB445. Replacement of the setA locus was confirmed by Southern hybridization using a probe annealing in the $3^{\prime}$ flanking region of SetA and restriction with $E c o R$ V. The resulting strain was named AGB715. For the expression of $s e t A:: g f p, g f p$ was amplified from pME3929 with the

primers OZG207 and OZG504. The setA gene was amplified from genomic DNA with the primers RH214b and RH222. The two fragments were fused by fusion PCR (Szewczyk et al., 2006) with the primers RH214b and OZG504. The amplicon was ligated into the SwaI restriction site of pME3160, resulting in pME4070. The 
plasmid was transformed into AGB152 resulting in AGB716. Ectopical integration as well as the integrity of the nitrate reductase locus was confirmed by Southern hybridization analysis with a nitrate promoter probe (see above) and restriction with XhoI.

\subsection{Construction of the $\operatorname{dcn} A, A N 4149$ and $A N 4491$ deletion strains}

For the construction of the $d c n A$ deletion cassette the 5' flanking region of the gene was amplified with the primers RH11 and RH12 and the 3' flanking region with RH15 and RH16 from genomic DNA. The ptrA resistance marker was amplified with RH13 and RH14 from pME3024. All three amplicons were fused by fusion PCR (Szewczyk et al., 2006) with the primers RH11 and RH16 and the cassette was transformed into the A. nidulans strain TNO2A3 resulting in strain AGB504 (von Zeska Kress et al., 2012). The deletion strain was verified by Southern hybridization using the 3' flanking region as probe and restriction with EcoRI, XhoI or ScaI. For construction of the AN4149 deletion strain the 5' flanking region was amplified using the primers JS1 and JS2 and the 3' flanking region was amplified with JS5 and JS6 from genomic DNA of a wild type. As marker, ptrA was used, generated by PCR with JS3 and JS4. The three amplicons were fused using JS1 and JS6. The cassette was subcloned into pJET1.2/blunt resulting in pME4065. For Aspergillus transformation in TNO2A3 the cassette was restricted from the plasmid using $P v u \mathrm{I}$ resulting in AGB738. Replacement of the gene by the ptrA marker cassette was confirmed by Southern hybridization using the $5^{\prime}$ flanking region as probe and AgeI restriction. For construction of the AN4491 deletion strain, the 5' flanking region and the 3' flanking region were amplified with JS9 and JS10 and JS13 and JS14, respectively. The ptrA marker was obtained by PCR with JS11 and JS12 and plasmid DNA of pME3024 as template. The amplicons were fused by fusion PCR (Szewczyk et al., 2006) using JS9 and JS14. The cassette was subcloned into pJET1.2/blunt resulting in pME4069. The deletion cassette was restricted from the plasmid using HindIII and AseI and transformed in TNO2A3 resulting in AGB739. Correct integration of the marker into the genome was verified using the 5' flanking region of AN4491 as probe and restriction with $B g l \mathrm{II}$. 


\subsection{Construction of the $r b x A$ deletion cassette and heterokaryon rescue}

For the construction of the $r b x A$ deletion cassette the $5^{\prime}$ and $3^{\prime}$ flanking regions were amplified from genomic DNA of a wild type strain with the primers RH43 and RH44 or RH47 and RH48, respectively. Additionally, the pyrG marker from A. fumigatus was amplified with RH45 and RH46. The three amplicons were fused by fusion PCR (Szewczyk et al., 2006) using the primers RH43 and RH48. The deletion cassette was transformed into the strain TNO2A3.

After three transformations that generated primary transformants but no surviving deletion strain the heterokaryon rescue technique was applied (Osmani et al., 2006). Assuming the deleted gene is essential, primary transformants grow as a heterokaryon, carrying nuclei with the wild type gene and nuclei carrying the $p y r G$ marker cassette instead of the gene in one hypha. As first indication for an essential gene, a growth test was performed. Spores from the primary transformants were replica streaked onto new selective and non-selective agar plates and incubated for $48 \mathrm{~h}$ at $37^{\circ} \mathrm{C}$. Asexual spores of $A$. nidulans only contain a single nucleus. Hence, on selective medium, wild type spores cannot germinate because of the missing pyrG marker, while spores with the marker integrated at the respective ORF cannot grow because of the essential function of the gene. In contrast, on non-selective medium, the wild type spores from the heterokaryon germinate and form a mycelium.

The next step was to proof the occurrence of the heterokarya. This was done by PCR with primers RH66 and RH67, annealing in the genes flanking region and outside of the deletion cassette, generating amplicons of different sizes for the wild type gene and the integrated deletion cassette. Heterokarya show both amplicons, while a deletion strain would show only the signal of the integrated deletion cassette and strains with ectopical integration of the cassette would show the wild type PCR fragment (von Zeska Kress et al., 2012).

\subsection{Plasmid and strain construction for DcnA BiFC experiments}

The cDNA of $d c n A$ was amplified with RH17 and RH18 and $r b x A$ cDNA with MK97 and MK98 from an A. nidulans cDNA pool and ligated into the pJET 1.2/blunt vector resulting in the plasmids pME3671 and pME3672, respectively. For construction of the plasmids for BiFC the cDNA of $d c n A$ was amplified from pME3672 with the primers RH97 and RH98 and fused to the N-terminal part of eyfp 
amplified from pME3012 with the primers RH99 and OLKM91 by fusion PCR (Szewczyk et al., 2006). The construct was cloned into a bidirectional niiA/niaD promoter containing plasmid pME3160 using the PmeI site resulting in plasmid pME3673. The cDNAs of $r b x A$, neddH and $u b c L$ were amplified from plasmids pME3671, pME3005 and pME3678 with the following primers: $r b x A-\mathrm{RH} 101$ and MK30, neddH-MK98 and RH100, ubcL-MK96 and RH102. The fragments were fused to the C-terminal part of eyfp amplified from pME3013 with the primers OLKM86 and OLKM87 by fusion PCR (Szewczyk et al., 2006). The fusion constructs were ligated into pME3673 using the $S w a I$ restriction site resulting in the plasmids pME3674 (eyfp::rbxA), pME3675 (eyfp::neddH) and pME3676 $($ eyfp::ubcL), respectively. As control, the C-terminal part of eyfp alone was ligated into pME3673 using the $S w a I$ restriction site resulting in pME3677. The plasmids were transformed into strain AGB152 resulting in AGB500, AGB501, AGB502 or AGB503, respectively (von Zeska Kress et al., 2012). Ectopical integration as well as the integrity of the nitrate reductase locus was confirmed by Southern hybridization analysis with a nitrate promoter probe (see above) and restriction with HincII.

\subsection{Strain construction for $f b o x 15:: g f p$ overexpression}

For the construction of strains, ectopically expressing high amounts of the fbox15::gfp fusion, the plasmid pME3950 was transformed into the A.nidulans strains AGB152 and AGB209. The resulting strains, AGB678 and AGB679, were verified by Southern hybridization using a probe annealing at fbox 15 and restriction with MseI. Deletion of $\operatorname{csn} E$ was confirmed by Southern hybridization using SspI restriction and a 5' flanking region as probe. High and similar expression of $f b x 15:: g f p$ RNA was confirmed by qRT-PCR with the primers JS110 and JS111 (von Zeska Kress et al., 2012). 


\subsection{Methods}

\subsubsection{Genetic manipulations and molecular methods}

\subsubsection{Transformation procedures}

E. coli cells were transformed as described before (Inoue et al., 1990). Transformations of A. nidulans were performed following the standard protocol (Eckert et al., 2000).

\subsubsection{Sequence analyses}

DNA was sequenced at the Göttingen Genomics Laboratory. Sequences were analyzed using the Lasergene (DNA STAR INC., Madison, WI, USA) and the 4 Peaks (MeKentosj, Amsterdam, NL) software. Gene and protein accession numbers were obtained from National Center for Biotechnology Information (NCBI; http://www.ncbi.nlm.nih.gov/). BLAST searches were performed at NCBI and the Aspergillus Genome Database (AspGD) (Arnaud et al., 2012) (http://www.aspgd.org). A. nidulans proteins were identified using AspGD or the Central Aspergillus ㅁata Repository (CADRE) (Mabey Gilsenan et al., 2012) (http://www.cadre-genomes.org.uk) genome browser. Protein domains were analyzed by InterProScan (Hunter et al., 2012, Quevillon et al., 2005) (http://www.ebi.ac.uk/Tools/pfa/iprscan). Sumoylation and reversed sumoylation motifs were identified using the phosida posttranslational modification database (Gnad et al., 2007, Gnad et al., 2011) (http://www.phosida.com). Sumo interaction motifs were predicted using the web tool of the University of Dundee (http://www.lifesci.dundee.ac.uk/groups/ron_hay/pages/SIMmotifQuery.html). Gene and protein information of $S$. cerevisiae was obtained from the Saccharomyces Genome Database (SGD) (Cherry et al., 2012) (http://www.yeastgenome.org). For information on S. pombe genes and proteins, PomBase (Wood et al., 2012) was used (http://www.pombase.org). Automated structure homology-modeling was conducted using the SWISS model server (http://swissmodel.expasy.org) (Arnold et al., 2006, Bordoli et al., 2009, Kiefer et al., 2009). Graphical illustration of structural data was performed with PyMol (The PyMOL Molecular Graphics System, Version 1.5.0.4 Schrödinger, LLC) (http://www.pymol.org). Alignments and calculation of protein similarity were done with Geneious (BIOMATTERS, Auckland, NZ). 


\subsubsection{Recombinant DNA methods}

In general, recombinant DNA methods were applied according to standard protocols (Sambrook et al., 1989). For PCR the following polymerases were used: Taq (Fermentas GmbH/Thermo Fisher ScIentific GmbH, St. Leon-Rot, D/Schwerte, D), Pfu (Fermentas GmbH/Thermo Fisher Scientific GmbH, St. Leon-Rot, D/ Schwerte, D) or Phusion High-Fidelity (FInNZYMES/THERMo FISHER SCIENTIFIC GMBH, Vantaa, FIN/Schwerte, D). Restriction enzymes, T4 polynucleotide kinase, T4 ligase and shrimp alkaline phosphatase were purchased from Fermentas GmbH/Thermo Fisher ScIEntific GmbH (St. Leon-Rot, $\mathrm{D} /$ Schwerte, D). All reactions were carried out according to manufacturer's instructions.

\subsubsection{DNA isolation and hybridization}

Plasmid DNA from E. coli was isolated using the QIAGEN Plasmid Plus Midi Kit or QIAGEN Plasmid Mini Kit (QIAGEN GMBH, Hilden, D), respectively, according to the producer's manual. DNA extraction from gel was performed using the QIAquick Gel Extraction Kit (QIAGEN GmbH, Hilden, D). Genomic DNA of A. nidulans was isolated from ground cell material as described previously (Lee \& Taylor, 1990). Southern hybridization (Southern, 1975) was carried out with nonradioactive probes using the AlkPhos Direct $^{\mathrm{TM}}$ labeling and the CDP-Star ${ }^{\mathrm{TM}}$ detection system from GE HealthCARE LIFE SCIENCE (München, D) following the manufactures protocols. Chemiluminescent signals were detected on Hyperfilm ${ }^{\mathrm{TM}}$ ECL (GE HEALTHCARE LTD., Little Chalfont, UK).

\subsubsection{RNA isolation and cDNA synthesis}

Extraction of total RNA from ground A. nidulans mycelia was carried out using the RNeasy plant kit (QIAGEN GMBH, HILDEN, D) according to the manufacture's instructions. DNase digestion followed by cDNA synthesis was conducted using $0.8 \mu \mathrm{g}$ of RNA and the QuantiTect Reverse Transcription kit (QIAGEN GMBH, HILDEN, D) following the manufacturers protocol. 


\subsubsection{Quantitative real-time PCR}

qRT PCRs were performed as described previously (von Zeska Kress et al., 2012). qRT PCR was performed with a LightCycler 2.0 (RoCHE GMBH, Mannheim, D) and the RealMaster SYBR Rox kit (5 PRIME GMBH, Hamburg, D). For each reaction, appropriate amounts of cDNA and gene-specific primers were used. The amplification conditions were as follows: 36 cycles of $15 \mathrm{~s}$ at $95^{\circ} \mathrm{C}, 22 \mathrm{~s}$ at $70^{\circ} \mathrm{C}, 22 \mathrm{~s}$ at $70^{\circ} \mathrm{C}$ and an adjacent melting step $\left(42^{\circ} \mathrm{C}-95^{\circ} \mathrm{C}\right)$. To quantify the relative amount of the gene of interest relative to histone $h 2 a$ RNA, the $\Delta c t$ method with efficiency (Pfaffl et al., 2002) was used. All qRT PCR experiments were performed at least in duplicate.

\subsubsection{Protein methods}

\subsubsection{Protein isolation and analyses}

Experiments were performed as described previously (von Zeska Kress et al., 2012, Christmann, 2011). A. nidulans strains were grown under the indicated time periods and conditions. Mycelia were harvested using Miracloth filters (MERCK KGAA, Darmstadt, D), washed with $0.96 \% \mathrm{NaCl}$, dried with paper towels and frozen in liquid nitrogen. The material was ground with mortar and pestle or a RETSCH MM400 ball mill (RETSCH GMBH, Hann, D) cooled with liquid nitrogen. To extract the proteins, B-buffer [300 mM NaCl, $100 \mathrm{mM}$ Tris- $\mathrm{HCl} \mathrm{pH} 7.2,10 \%$ glycerol, $0.1 \%$ NP-40, freshly added $1 \mathrm{mM}$ DTT, PIM] was added to the mycelia and mixed until a homogenous, viscous suspension was achieved. After centrifugation with 16,000 rcf at $4{ }^{\circ} \mathrm{C}$ for $30 \mathrm{~min}$ the supernatant was used for further analyses. Protein concentrations were mesured as described before (Bradford, 1976) using the RotiQuant® solution (CARL RoTH GMBH \& Co. KG, Karlsruhe, D) following the manufactures instructions.

For immunoblotting, protein suspension was mixed with 3x sample buffer [250 mM Tris-HCl pH 6.8, 15\% $\beta$-mercaptoethanol, 30\% glycerol, 7\% SDS, $0.3 \%$ bromphenol blue] and denatured by incubation at $95^{\circ} \mathrm{C}$ for $10 \mathrm{~min}$. Samples were subjected to $5-20 \%$ gradient or $12 \%$ non-gradient SDS-PAGE and transfered to a nitrocellulose membrane (WhatMAN GMBH, Dassel, D). The PageRuler ${ }^{\mathrm{TM}}$ Prestained Protein Ladder (Fermentas GMBH/THERmo Fisher ScIENTIFIC GMbH, St. Leon-Rot, D/ Schwerte, D) was used as molecular weight marker. For detection 


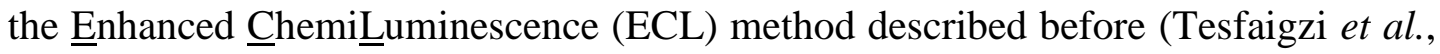
1994) was used. Signals were detected on Hyperfilm ${ }^{\text {TM }}$ ECL (GE HEALTHCARE LTD., Little Chalfont, UK) or with a Fusion-SL 4.2 MP system (Peqlab Biotechnologie GMBH, Erlangen, D). Signal intensity was quantified using the Fusion-SL 4.2 MP system with Bio1D software (PeQlab BioteChNologie GMBH, Erlangen, D).

\subsubsection{Antibodies}

As primary antibodies for GFP fusion protein a GFP antibody (sc-9996, SAnta CruZ Biotechnology Inc., Dallas, TX, USA) was used. TAP fusion proteins were detected with Calmodulin binding protein antibody (UPSTATE/Millipore, Billerica, MA, USA). Tubulin antibody (SigMA-AldRICH Chemie GmbH, Steinheim, D) or Actin antibody (Novus BiochemicAls, Littelton, CO, USA) was used as loading control. Polyclonal antibodies directed against the A. nidulans proteins NeddH, SumO and Ubiquitin were obtained by rabbit immunization with peptides of the respective proteins (GENSCRIPT USA INC., Piscataway, NJ, USA). HRP labeled mouse (115-035-003, JACKSON IMMUNO RESEARCH, Newmarket, UK) and rabbit (G21234, InVITROGEN GMBH, Karlsruhe, D) secondary antibodies were used.

\subsubsection{Tandem Affinity Purification (TAP)}

TAP experiments were performed as described previously (Bayram et al., 2008b, Busch et al., 2007, Christmann, 2011, Helmstaedt et al., 2008). Proteins from $101-151$ culture were extracted (see chapter 2.4.1). $50 \mathrm{ml}$ portions of the crude extracts were incubated with $400 \mu \mathrm{l}$ IgG-agarose (GE HEALTHCARE LifE SCIENCE, München, D) for $2 \mathrm{~h}$ on a rotating platform at $4^{\circ} \mathrm{C}$. The suspension was subjected to a PolyPrep column (BIO-RAd LABORATORIEs GMBH, München, D). Beads with bound TAP-tagged and interacting proteins remained in the column, while the flowthrough contained unbound proteins. Beads were washed twice with $10 \mathrm{ml}$ IPP300 (25 mM Tris-HCl pH 8.0, $300 \mathrm{mM} \mathrm{NaCl}, 0.1 \%$ NP-40, $2 \mathrm{mM}$ DTT) and once with $10 \mathrm{ml}$ IPP150 (25 mM Tris-HCl pH 8.0, $150 \mathrm{mM} \mathrm{NaCl}, 0.1 \%$ NP-40, 2 mM DTT). After another washing step with $10 \mathrm{ml}$ tobacco etch virus (TEV) cleavage buffer (TEV-CB: $25 \mathrm{mM}$ Tris-HCl pH 8.0, $150 \mathrm{mM} \mathrm{NaCl,} \mathrm{0.1 \%} \mathrm{NP-40,} 0.5 \mathrm{mM}$ EDTA, $1 \mathrm{mM}$ DTT), the columns were closed. $1 \mathrm{ml}$ of TEV-CB and 300 units TEV protease 
were added to the beads. The samples were incubated on a rotating platform for overnight at $4^{\circ} \mathrm{C} .300 \mu \mathrm{l}$ calmodulin affinity resin (AGILENT TECHNOLOGIES GMBH, Waldbronn, D) were equilibrated with $5 \mathrm{ml}$ of calmodulin binding buffer (CBB; $25 \mathrm{mM}$ Tris- $\mathrm{HCl} \mathrm{pH}$ 8.0, $150 \mathrm{mM} \mathrm{NaCl}, 1 \mathrm{mM}$ Mg-acetate, $1 \mathrm{mM}$ imidazole, $2 \mathrm{mM}$ $\mathrm{CaCl}_{2}, 10 \mathrm{mM}$ B-mercaptoethanol). Proteins from the TEV cleavage reaction were eluted in two steps with $1 \mathrm{ml}$ of TEV-CB each and collected in the calmodulin beads containing PolyPrep columns. $6 \mathrm{ml} \mathrm{CBB}$ with $6 \mu \mathrm{l}$ of $1 \mathrm{M} \mathrm{CaCl}_{2}$ were added to the eluate. After incubation on a rotating platform for $2 \mathrm{~h}$ at $4^{\circ} \mathrm{C}$, beads were washed twice with $1 \mathrm{ml}$ of $\mathrm{CBB}$ containing $0.1 \% \mathrm{NP}-40$ and once with $1 \mathrm{ml}$ of $\mathrm{CBB}$ containing $0.02 \%$ NP-40 to remove unbound proteins. Bond proteins were eluted with $1 \mathrm{ml}$ of calmodulin elution buffer $(25 \mathrm{mM}$ Tris- $\mathrm{HCl} \mathrm{pH} 8.0,150 \mathrm{mM} \mathrm{NaCl}$, 0.02\% NP-40, $1 \mathrm{mM}$ Mg-acetate, $1 \mathrm{mM}$ imidazole, $20 \mathrm{mM}$ EGTA, freshly added: $10 \mathrm{mM}$ ß-mercaptoethanol). This step was repeated twice. Eluted proteins were precipitated with trichloroacetic acid (TCA) in an overnight incubation at $4^{\circ} \mathrm{C}$. Proteins were collected by centrifugation with $16,000 \mathrm{rcf}$ for $1 \mathrm{~h}$ at $4^{\circ} \mathrm{C}$. The precipitate was washed with ice-cold acetone/ $0.05 \mathrm{~N} \mathrm{HCl}$ and with acetone. Precipitated proteins were dried in a vacuum exhausted centrifuge. The proteins were resuspended in $30 \mu 13 x$ sample buffer (see chapter 2.2.2.1.) and separated on SDSPAGE. Gels were stained with Coomassie brilliant blue (SERVA ElECTROPHORESIS GMBH, Heidelberg, D) (see chapter 2.2.2.4.) and bands were excised for tryptic digestion (see chapter 2.2.2.5.) and subsequent LC-MS analysis (see chapter 2.2.2.6.).

\subsubsection{Coomassie staining}

Proteins on SDS-PAGE were stained with Coomassie brilliant blue stain [40\% v/v ethanol, 10\% v/v acetic acid, $1 \mathrm{~g}$ Coomassie brilliant blue R250 (SERVA ElECTROPHORESIS GMBH, Heidelberg, D)] by incubation at room temperature (RT) for 15 min with agitation. Destaining was carried out in $12.5 \% \mathrm{v} / \mathrm{v}$ isopropanol/ $10 \%$ $\mathrm{v} / \mathrm{v}$ acetic acid at RT.

\subsubsection{Tryptic in-gel digestion of protein samples}

Tryptic digestion of the protein samples was carried out as described before (Christmann, 2011, Shevchenko et al., 1996, von Zeska Kress et al., 2012). Briefly, Coomassie-stained gel pieces for tryptic in-gel digestion were excised and cut into 
small pieces. Acetonitrile was added to the gel slices and samples were incubated for $10 \mathrm{~min}$ at RT. The liquid was removed and the pieces were dried in a vacuum exhausted centrifuge. $150 \mu \mathrm{l}$ of $10 \mathrm{mM}$ DTT in $100 \mathrm{mM} \mathrm{NH} \mathrm{HCO}_{3}$ were added to the samples, followed by incubation at $56^{\circ} \mathrm{C}$ for $1 \mathrm{~h}$. The DTT solution was removed

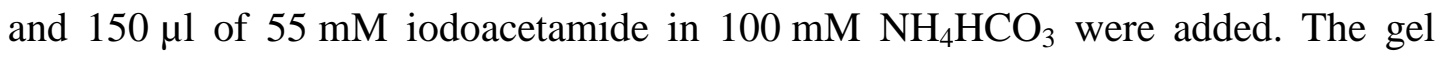
pieces were incubated at RT for 45 min in darkness. The iodoacetamide solution was removed and $150 \mu \mathrm{l}$ of $100 \mathrm{mM} \mathrm{NH} \mathrm{NCO}_{3}$ were added. After 10 min incubation at RT the $\mathrm{NH}_{4} \mathrm{HCO}_{3}$ was replaced by $150 \mu \mathrm{l}$ acetonitrile. Both steps were repeated once and the gel pieces were dried in a vacuum exhausted centrifuge. Trypsin digestion buffer [1:20 sequencing grade modified trypsin (V5111, Promega GMBH, Mannheim, D) in $50 \mathrm{mM} \mathrm{NH} \mathrm{HCO}_{3}$ ] was added and the samples were incubated on ice for $45 \mathrm{~min}$. Excessive digestion buffer was removed, samples were covered with $50 \mathrm{mM} \mathrm{NH} \mathrm{HCO}_{3}$ and incubated overnight at $37^{\circ} \mathrm{C}$. The next day, gel pieces were centrifuged at 11,000 rcf at RT for $1 \mathrm{~min}$. The supernatant was collected and $20 \mathrm{mM}$ $\mathrm{NH}_{4} \mathrm{HCO}_{3}$ was added to the samples. The gel pieces were incubated for $10 \mathrm{~min}$ at $\mathrm{RT}$, the liquid was collected and a $50 \%$ acetonitril/5\% formic acid solution was added to the samples. After a $20 \mathrm{~min}$ incubation step at RT, samples were centrifuged (11,000 rcf, $1 \mathrm{~min}, \mathrm{RT})$ and the supernatant was collected. The extraction steps were repeated twice, supernatants were pooled per sample and the collected liquid was evaporated in a vacuum exhausted centrifuge. Precipitated proteins were reconstituted in $20 \mu 195 \% \mathrm{H}_{2} \mathrm{O} / 5 \%$ acetonitrile/0.1\% formic acid and subjected to LC-MS analysis (see chapter 2.2.2.6.).

\subsubsection{Protein identification by mass spectrometry}

\subsection{NanoLC-NanoESI mass spectrometry analyses and database search (TAP::SumO)}

Peptides of $2 \mu \mathrm{l}$ sample solution from the trypsin digested proteins were trapped and washed with $0.05 \%$ trifluoroacetic acid on an Acclaim ${ }^{\circledR}$ PepMap 100 column $(75 \mu \mathrm{m}$ x $2 \mathrm{~cm}, \mathrm{C} 18,3 \mu \mathrm{m}, 100 \AA \AA$, P/N164535 THERMO FiSHER SCIENTIFIC GMBH, Schwerte, D) at a flow rate of $4 \mu \mathrm{l} / \mathrm{min}$ for $12 \mathrm{~min}$. Analytical peptide separation by reverse phase chromatography was performed on an Acclaim ${ }^{\circledR}$ PepMap RSLC column $(75 \mu \mathrm{m} \times 15 \mathrm{~cm}, \mathrm{C} 18,3 \mu \mathrm{m}, 100 \AA$ A, P/N164534 THERMO FISHER SCIENTIFIC GMBH, Schwerte, D) running a gradient from $96 \%$ solvent A $(0.1 \%$ 
formic acid) and $4 \%$ solvent $\mathrm{B}$ (acetonitrile, $0.1 \%$ formic acid) to $50 \%$ solvent $\mathrm{B}$ within $25 \mathrm{~min}$ at a flow rate of $250 \mathrm{nl} / \mathrm{min}$ (solvents and chemicals: THERMO FISHER SCIENTIFIC GMBH, Schwerte, D). Peptides eluting from the chromatographic column were on-line ionized by nano-electrospray (nESI) using the Nanospray Flex Ion Source (Thermo Fisher ScIENTIFIC GMBH, Schwerte, D) and transferred into the mass spectrometer. Full scans within $\mathrm{m} / \mathrm{z}$ of $300-1850$ were recorded by the Orbitrap-FT analyser at a resolution of 60,000 at $\mathrm{m} / \mathrm{z} 400$. Peptides were fragmented by collision-induced decay (CID) in the LTQ Velos Pro linear ion trap. LC/MS method programming and data acquisition was performed with the software XCalibur 2.2 (Thermo Fisher ScIENTIFIC GMBH, Schwerte, D).

Orbitrap raw files were analyzed with the Proteome Discoverer 1.3 software (Thermo Fisher ScIENTIFIC GMBH, Schwerte, D) using the Mascot and Sequest search engines against the $A$. nidulans protein database with the following criteria: peptide mass tolerance $10 \mathrm{ppm}$; MS/MS ion mass tolerance $0.8 \mathrm{Da}$ and up to two missed cleavages allowed. The variable modification considered was methionine oxidation and carbamidomethylation was considered as fixed modification. For protein identification at least two different high ranking peptides were required that after validation against a decoy database had a false discovery rate of 0.01 or less (published in (Harting et al., 2013)).

\subsection{Mass spectrometry analyses (TAP::SetA)}

Analyses were carried out as described before (Christmann, 2011, von Zeska Kress et al., 2012). Peptides originating from in-gel trypsin digested protein bands were loaded on a NAN75-15-03-C18-PM column with an ultimate3000 HPLC system (Dionex CORPORATION, Sunnyvale, CA, USA). Mass analyses were carried out with a LCQ DecaXP mass spectrometer (THERMO FISHER SCIENTIFIC GMBH, Schwerte, D). Cycles of MS spectra with $\mathrm{m} / \mathrm{z}$ ratios of peptides and four datadependent MS2 spectra were recorded. The 'peak list' was created with Extractms from the Xcalibur software package (BioworksBrowser 3.3.1SP1). The MS2 spectra were aligned with the $A$. nidulans genome database (http://www.aspergillusgenomes.org) with the Turbo-SEQUEST program (Lundgren et al., 2005) from Bioworks (Thermo Fisher ScIENTIFIC GMBH, Schwerte, D). Protein identification required a high scoring peptide with the following criteria: (i) 
$\mathrm{XCorr}(1+, 2+, 3+)>2.0,2.5,3.0$, (ii) $\Delta \mathrm{Cn}>0.4$ and (iii) $\mathrm{Sp}>500$. MS2 spectra of the highest scoring peptides were individually verified.

\subsubsection{Protein stability assay using cycloheximide}

Strains were grown under vegetative conditions for $20 \mathrm{~h}$ in liquid $\mathrm{MM}$ at $37^{\circ} \mathrm{C}$. The mycelium was harvested by filtration through a sterile miracloth filter, washed with $0.96 \% \mathrm{NaCl}$ solution and further incubated in liquid $\mathrm{MM}$ containing $25 \mu \mathrm{g} / \mathrm{ml}$ cycloheximide for 1,2 and $4 \mathrm{~h}$ at $37^{\circ} \mathrm{C}$ (von Zeska Kress et al., 2012).

\subsubsection{Microscopy and quantification}

\subsubsection{Microscopic analyses}

A. nidulans colonies, hyphae and developmental structures were pictured with a CS30 digital camera (Olympus GMBH, Hamburg, D) and an OlYMPUS SZXILLB2-200 binocular or an Axiolab microscope (CARL ZeISs GMBH, Jena, D). The KL1500-LCD light source (Olympus GMBH, Hamburg, D) was used for illumination of samples observed with the binocular. Pictures were taken and calibrated for magnification with the cellSens software (OLYMPUS GMBH, Hamburg, D). For observation of conidiophores, $5 \mathrm{ml}$ of $\mathrm{MM}$ containing $2 \%$ agar and appropriate supplements were applied onto oblique glass slides placed in petri dishes and inoculation was performed with $2 \times 10^{4}$ spores (Laubinger, 2008). Strains were grown at $37^{\circ} \mathrm{C}$ for the indicated time.

For fluorescent microscopy a ZEISS Axio Observer Z.1 system (CARL ZEISS GMBH, Jena, D) with a QuantEM:512SC (Photometrics, Tucson, AZ, USA) camera or a Coolsnap HQ ${ }^{2}$ (PhOtometrics, Tucson, AZ, USA) and the SlideBook 5.0 imaging software (InTELLIGENT IMAGING InNOVATIONS INC., Denver, CO, USA) was used. For BiFC experiments a ZEISS 100 Axiovert microscope (CARL ZEISS GMBH, Jena, D) was used and photographs were taken with a XILLIX Microimager digital camera (XILIX TeCHNOLOGIES CORP., Richmond, CAN) and the Openlab 5.01 software (IMPROVISION, Coventry, UK).

For fluorescent microscopy, $2 \times 10^{5}$ spores of the corresponding strains were added to $400 \mu 1$ liquid MM medium in an eight-well chambered coverslip (NUNC GMвH \& Co. KG, Langenselbold, D) and incubated at $37^{\circ} \mathrm{C}$ under approptiate 
conditions. Alternatively, $500 \mu \mathrm{l}$ of the appropriate medium on cover slips, placed in petri dishes, was used and inoculated with $4 \times 10^{4}$ spores. After incubation at the respective temperature, cover slips were fixed on glass slides with nail polish

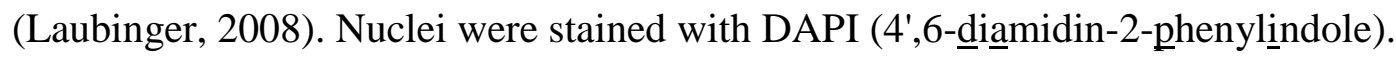

\subsubsection{Quantification methods}

Colony growth was measued daily as colony diameter and quantification of conidiospores was performed as described previously (Busch et al., 2003). Spores were counted with a counting chamber or a Coulter Z2 particle count and size analyzer (BECKMANn COULTER GMBH, Krefeld, D).

\subsubsection{Analyses of sterigmatocystin production}

For the analyses of the sterigmatocystin production, $1 \times 10^{7}$ spores of the respective strains were grown on oat meal medium [minimal medium with $24 \mathrm{~g} / 1$ oatmeal ('Holo Bio Hafergold', NeUform, Zarrentin, D)] for $4 \mathrm{~d}$ at $37^{\circ} \mathrm{C}$. Agar pieces of around $1 \mathrm{~cm}^{2}$ were cut into small slices and mixed with glass beats and $3 \mathrm{ml}$ water for $30 \mathrm{~min}$. To break the cells, samples were treated with sonication for $1 \mathrm{~min}$ with a Sonoplus device (BANDELIN ELECTRONICS, Berlin, D). After addition of $3 \mathrm{ml}$ chloroform, the samples were mixed for more $30 \mathrm{~min}$ at $4^{\circ} \mathrm{C}$ and centrifuged at $4^{\circ} \mathrm{C}$ for $10 \mathrm{~min}$. The lower phases were then transferred to screw-cap glasses. The solvent was removed and the remaining metabolites were dissolved in $50 \mu 1$ methanol. The samples were subjected to thin layer chromatography. For this, $20 \mu \mathrm{l}$ were transferred to pre-coated TLC-sheets Polygram SIL G/UV 254 (MACHEREY-NAGEL GMBH, Düren, D). As standard, $2 \mu \mathrm{l}$ of a $1 \mathrm{mg} / \mathrm{ml}$ solution sterigmatocystin (ApPlChem GmBH, Darmstadt, D) was applied. As eluent a mixture of acetone/chloroform (ratio 1:4) was used. The chromatography plate was dried and sprayed with $\mathrm{AlCl}_{3}$ solution ( $5 \% \mathrm{AlCl}_{3}$ in $98 \%$ ethanol). The substance was pictured in a TLC visualizer (CAMAG, Berlin, D). 


\section{Results}

\subsection{The Neddylation network in Aspergillus nidulans}

\subsubsection{DenA as NeddH E3 ligase}

\subsubsection{DenA interacts with the neddylation machinery in vivo}

Neddylation is an important cellular process to regulate the activity of SCF Ubiquitin E3 ligases and therefore the protein degradation by the $26 \mathrm{~S}$ proteasome. The yeast protein Dcn1p was shown to act as a Nedd8 (Rub1p) E3 ligase in cullin neddylation (Kurz et al., 2008). To investigate the function of this protein in the neddylation network of the filamentous fungus, A. nidulans Dcn1 was identified using BLAST search with the human and the Saccharomyces cerevisiae protein sequence as query against the Aspergillus nidulans genome database (Galagan et al., 2005). The potential homolog, AN7259, has a size of 268 amino acids. It shows around $20 \%$ amino acid identity to yeast and around $30 \%$ identity to human Den1. The overall domain structure of the protein is conserved. Like other Den1 proteins, AN7259 contains an UBA-like domain at its N-terminus, while a so-called PONY domain is located C-terminally. According to the similarities and A.nidulans nomenclature, the identified gene was named $\operatorname{dcn} A$ and the corresponding protein DenA.

It was verified that DcnA is the Dcn1 homolog by studying its interactions in vivo by bimolecular fluorescence complementation (BiFC) (Hu et al., 2002). A plasmid was constructed, carrying a bidirectional inducible nitrate promoter. The $d c n A$ cDNA was fused to the N-terminal part of eyfp, whereas neddH, $r b x A$ (RING) and $u b c L$ (E2 enzyme) cDNAs were fused to the C-terminal part and inserted into the vector. Only interacting proteins generate an eYFP fluorescent signal. As control, a plasmid containing the $d c n A:: n y f p$ fusion and non-fused $c y f p$ was constructed. The plasmids were ectopically integrated into an A.nidulans wild type strain and subjected to fluorescence microscopy. Under promoter inducing growth conditions (nitrate) a fluorescence signal in the cytoplasm as well as a signal in the nucleus of the cell was observed (Figure 10), suggesting specific interactions between DcnA and RbxA as well as with NeddH and the NeddH E2 enzyme UbcL. In contrast, the control strain, expressing the $d c n A:: y f p$ fusion and the C-terminal half of $y f p$ did not show any fluorescence signal (data not shown), confirming signal specificity. This 
corroborates that DenA interacts in a similar way with the neddylation network as in other organisms (published in (von Zeska Kress et al., 2012)).

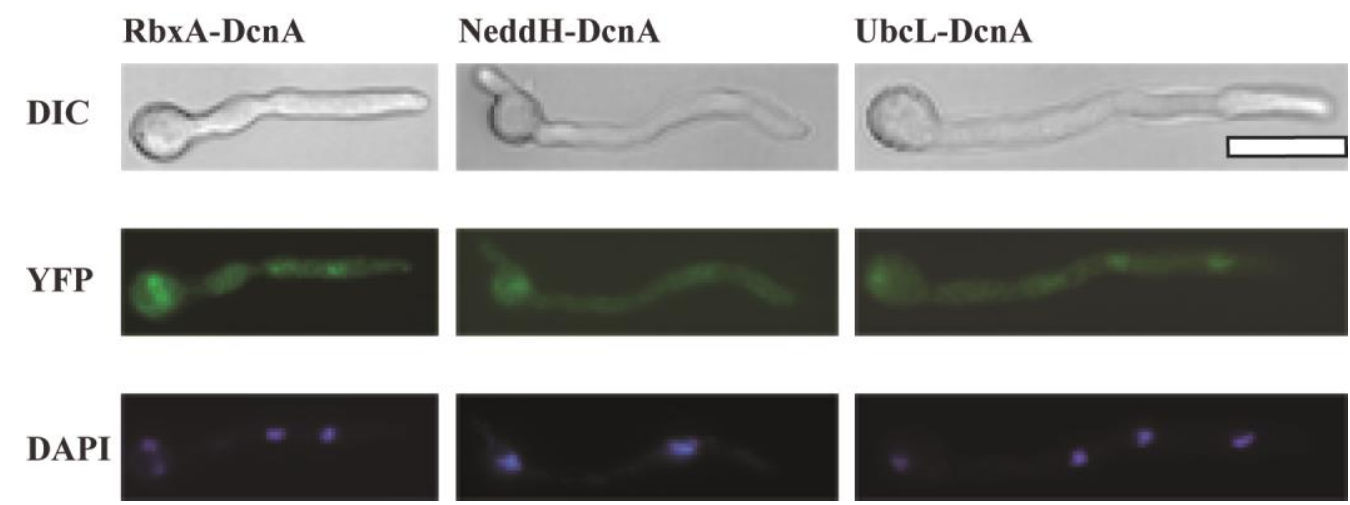

Figure 10: DenA interacts with components of the neddylation and ubiquitination machinery.

Interaction partners of DcnA visualized by bimolecular fluorescence complementation (BiFC). Indicated fungal strains were grown over night in 500 $\mu 1$ London medium with nitrate for induction of the corresponding promoters. DIC, YFP and DAPI counterstained pictures of the respective hyphae are shown. YFP signals correspond to interactions of the N-terminal eYFP fused to DenA with RbxA, NeddH, and UbcL fused to C-terminal eYFP. Scale bar $10 \mu \mathrm{m}$ (published in (von Zeska Kress et al., 2012)).

\subsubsection{Deletion of $\operatorname{dcn} A$ allows normal development while deletion of $r b x A$ is lethal}

In A. nidulans it was shown that neddH and parts of the neddylation and ubiquitination machinery, such as $u b c L, c u l A$, and $s k p A$ are essential for fungal viability (von Zeska Kress et al., 2012). A $d c n A$ deletion strain was constructed to analyze the phenotype of a strain, lacking the potential NeddH-E3 ligase. The coding region of the gene was replaced by a ptrA resistance cassette which was confirmed by Southern hybridization. The phenotype of the deletion strain was investigated. The growth of the $\Delta d c n A$ strain was comparable with the wild type. Neither in asexual nor in sexual development any obvious defects were observed. The strain formed normal developed cleistothecia under sexual development inducing conditions (Figure 11). The number of conidiospores produced in asexual development appeared to be similar to the wild type (Figure 11).

As there is the possibility that RbxA is the more important Nedd8-E3 ligase, while DcnA has an auxiliary function potentiating the reaction, the homolog of the RING in A.nidulans was investigated. BLAST search was applied in order to 
identify the Rbx1 homolog in the fungus, using the protein sequence of $S$. cerevisiae Rbx1p/Hrt1p as query. AN8844 showed around 90\% amino acid identity to the yeast and to the human protein. In addition, the potential A. nidulans homolog has a conserved RING finger motif characteristic for the protein family. According to the similarities and A. nidulans nomenclature, the identified gene was named $r b x A$ and the corresponding protein RbxA.
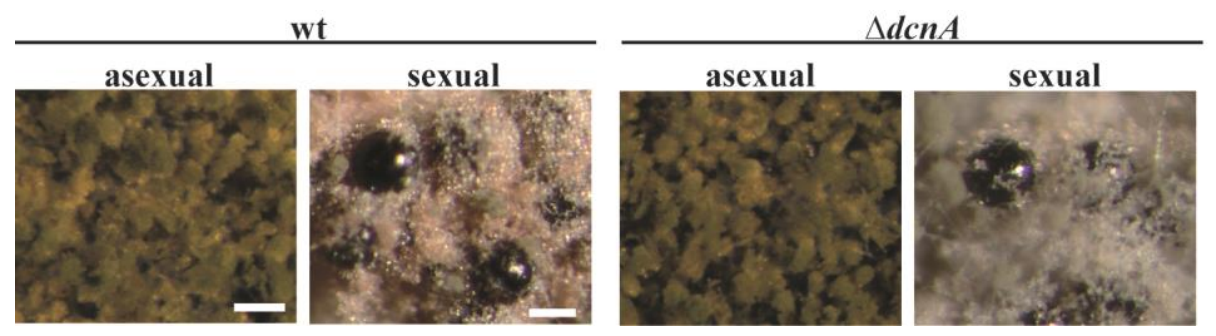

\section{Figure 11: Deletion of $d c n A$ allows normal development in $A$. nidulans.}

Asexual and sexual development of the wild type (wt) and the $d c n A$ deletion strain $(\Delta d c n A) .1 \times 10^{6}$ spores were evenly spread on minimal medium plates, containing appropriate supplements. Plates were incubated under asexual inducing conditions for 3 days at $37^{\circ} \mathrm{C}$ or under sexual inducing conditions for 10 days at $37^{\circ} \mathrm{C}$, respectively. Scale bar $100 \mu \mathrm{m}$.

An $r b x A$ deletion cassette was transformed into the $A$. nidulans strain TNO2A3. Heterokaryons could be obtained, containing two different types of nuclei with an intact copy of the gene and a copy of the inserted deletion cassette at the locus of the same gene (for details see 2.1.2.5.13.) which was confirmed by PCR and growth tests (data not shown). However, the homokaryon, containing only the nuclei with $r b x A$ replaced by the marker cassette, could not survive. This suggests that $r b x A$ is essential for fungal viability similar to other components of the ubiquitination and neddylation network in A. nidulans (published in (von Zeska Kress et al., 2012)).

\subsubsection{DenA functions as an auxiliary NeddH-E3 ligase}

The $d c n A$ deletion strain did not show any developmental defects in contrast to deletion strains of other components of the neddylation and ubiquitination machinery, although the protein interacts with NeddH, UbcL and RbxA. Therefore, it should be tested, whether DcnA supports the neddylation reaction as it was described in other organisms. Thus, the complete cellular protein from vegetative cultures of 
the $\triangle d c n A$ and a wild type strain grown at $37^{\circ} \mathrm{C}$ for $20 \mathrm{~h}$ was extracted and used for immunoblotting with a NeddH-specific antibody (Figure 12A).

A prominent band at the size range of neddylated cullins was observed. The three fungal cullins have a size of around 90 to $100 \mathrm{kDa}(\mathrm{CulA} 89 \mathrm{kDa}$, CulC $94 \mathrm{kDa}$, CulD $100 \mathrm{kDa}$ ). The molecular weight of NeddH-modified cullins increases the size by $9 \mathrm{kDa}$. As control and normalization signal tubulin antibody was used. A quantification of signal intensity showed that the $d c n A$ deletion strain moderately reduces the signal of neddylated cullins in comparison to wild type (Figure 12B). This suggests that DenA is an auxiliary NeddH-E3 ligase and that the capacity of the fungal neddylation machinery is sufficiently high to tolerate subtle reductions without an impact on fungal development (published in (von Zeska Kress et al., 2012)).

A

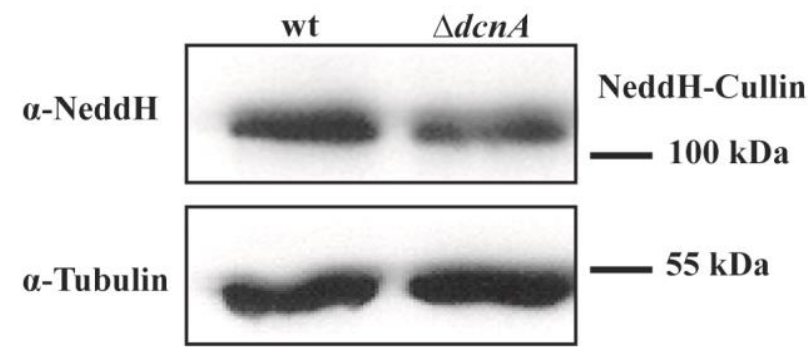

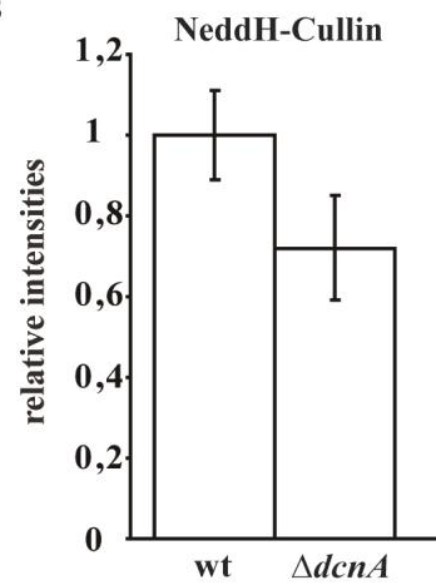

Figure 12: Deletion of $\operatorname{dcn} A$ moderately decreases the cellular levels of neddylated cullins.

A Neddylated proteins in the A. nidulans wild type (wt) and the $d c n A$ deletion strain $(\triangle d c n A)$. Immunoblot analyses using $50 \mu \mathrm{g}$ protein extract from strains, grown 20 hours in liquid culture at $37^{\circ} \mathrm{C}$ and a NeddH-specific antibody. Shown is the most intensive signal with a size, representing neddylated cullin. As control the same membrane was probed with $\alpha$-tubulin. B Quantification of signal intensities. The relative intensity of the signal of neddylated cullins of both strains was quantified. The signal intensity of the wild type corresponds to 1 . The signal of tubulin was used as internal standard to normalize signal intensities. Data are derived from three experiments with four replications each (published in (von Zeska Kress et al., 2012)).

\subsubsection{The CSN deneddylase stabilizes Fbox15}

The neddylation machinery, with the exception DcnA, is essential for fungal life. In contrast, deletions in the CSN deneddylase lead to a block in sexual 
development at the stage of primordia formation and changes in secondary metabolism (Braus et al., 2010, Busch et al., 2003, Busch et al., 2007). To visualize the changes in the neddylation pattern in A. nidulans upon defects in the CSN, the $\Delta c s n E$ strain together with the wild type was grown in vegetative culture over night at $37^{\circ} \mathrm{C}$ for $20 \mathrm{~h}$. Proteins were extracted from mycelia and used for immunoblotting with a NeddH-specific antibody and a tubulin antibody as control (Figure 13).

A

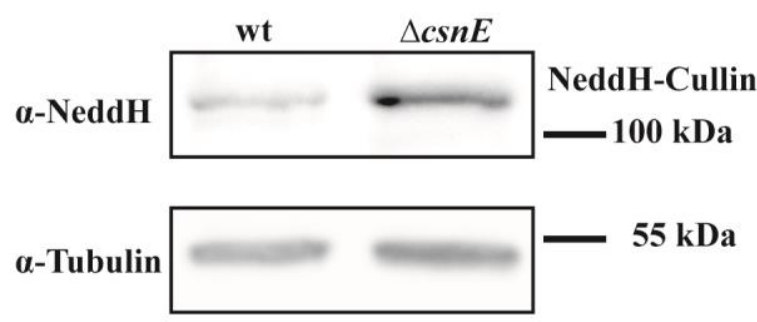

B

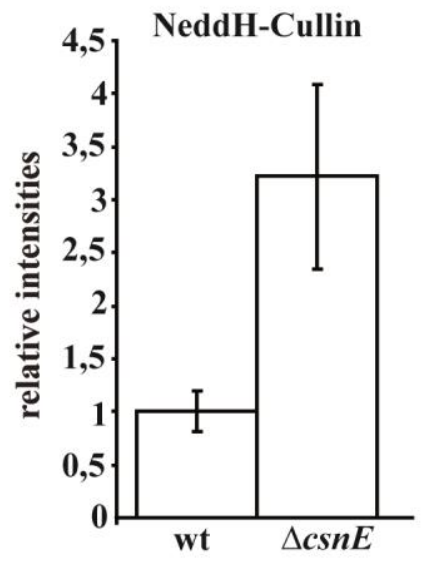

Figure 13: Deletion of the CSN deneddylase increases the levels of neddylated cullins.

A Neddylated proteins in the A. nidulans wild type (wt) and the $\operatorname{csn} E$ deletion strain $(\Delta \operatorname{csn} E)$. Immunoblot analyses using $50 \mu \mathrm{g}$ protein extract from strains, grown $20 \mathrm{~h}$ in liquid culture at $37^{\circ} \mathrm{C}$ and a NeddH-specific antibody. Shown is the most intensive signal, representing neddylated cullin. As control the same membrane was probed with tubulin antibody. B Quantification of signal intensities. The relative intensity of the signal of neddylated cullins of both strains was quantified. The signal intensity of the wild type corresponds to 1 . The signal of tubulin was used as internal standard to normalize signal intensities. Data are derived from two experiments with four replications each (published in (von Zeska Kress et al., 2012)).

The $\Delta \operatorname{csn} E$ strain displayed an increase in neddylated proteins compared to the wild type. A quantification of the signal intensity revealed an approximately three fold increase of neddylated cullins in the CSN deneddylase deficient strain. In a previous study, interacting proteins were purified expressing TAP-tagged $\mathrm{NeddH}$ in the wild type and the $\operatorname{csn} E$ deficient strain (von Zeska Kress et al., 2012). The neddylation machinery and the components of the SCF complex SkpA, cullins and the RING RbxA were indentified in both strains. In addition, several Fbox proteins were specifically co-purified in the $\operatorname{csn} E$ deletion strain. Furthermore it was shown that the deletion of some of these Fbox proteins, such as Fbox15, results in a strong developmental phenotype (von Zeska Kress et al., 2012). 
This raises the question, whether the accumulation of Fbox proteins results from a general increase in stability upon deletion of the deneddylase or in a specific stabilization of developmental SCF complexes containing Fbox15. In order to address this question, a GFP-tagged version of Fbox15 was ectopically expressed under control of the strong $g p d A$ promoter in the wild type and the $\operatorname{csn} E$ deletion strain. Similar levels of the $g f p:$ :fbox15 RNA were confirmed by qRT-PCR (data not shown). Next, proteins were extracted from vegetative cultures of the two strains and GFP-Fbx15 levels were compared by western hybridization using anti-GFP and antiactin antisera as control. Significant differences between the overall protein levels of GFP-Fbox 15 in the wild type and the $\operatorname{csn} E$ deletion strain were observed (Figure 14B). The levels of the fusion protein in the deneddylase deficient strain decreased to approximately $40 \%$ compared to the one in the wild type background (Figure 14, time point 0 ).

A

Time after cycloheximide treatment (h)
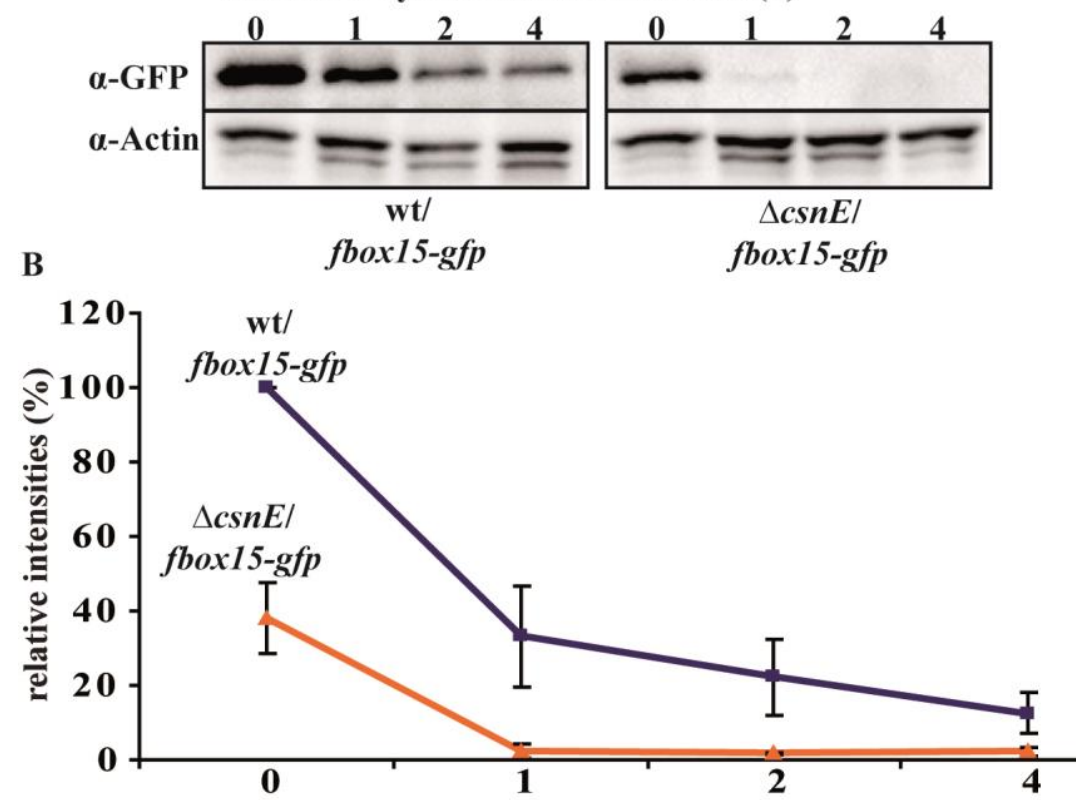

Time after cycloheximide treatment (h)

\section{Figure 14: The CSN stabilizes Fbox15-GFP.}

A Immunoblot of strains, ectopically expressing $f b x 15-g f p$ in wild type (wt/fbox15-gfp) and the $c s n E$ deletion ( $\Delta c s n E / f b o x 15-g f p)$ background using GFP antibody and actin antibody as control. Crude extracts were prepared from $20 \mathrm{~h}$ vegetative grown mycelium $(0 \mathrm{~h})$ and mycelium shifted to $25 \mu \mathrm{g} / \mathrm{ml}$ cycloheximide containing medium for 1, 2 and 4 hours (published in (von Zeska Kress et al., 2012)). B Quantification of A. Graphs show the relative intensities of the signals detected on the immunoblot normalized to actin levels. The wild type signal before cycloheximide treatment corresponds to $100 \%$. The decrease of signal intensities after cycloheximide treatment was visualized over time. Data is derived from two independent experiments. 
This suggests that CSN stabilizes Fbox15. In addition, protein synthesis was inhibited by the addition of cycloheximide. Fbox15-GFP levels were monitored at different time points. The Fbox15-GFP protein was significantly more stable in the wild type strain compared to the $\operatorname{csn} E$ deletion strain. Whereas in wild type background four hours after cycloheximide treatment approximately $15 \%$ of the fusion protein was still visible, only slight GFP-signals were detectable one hour after cycloheximide addition in the $\Delta \operatorname{csn} E$ strain.

These data further confirm the importance of CSN for the stability of Fbox 15 and therefore suggest that the Fbox proteins enriched in the TAP experiments belong to a subpopulation of substrate adaptors arrested in SCF complexes during development upon deletion of $\operatorname{csn} E$ (published in (von Zeska Kress et al., 2012)).

\subsubsection{The NeddH-associated proteins of unknown function AN4149 and AN4491 and fungal development}

Besides parts of the neddylation/ubiquitination machinery, other proteins were enriched with TAP::NeddH which potentially belong to the neddylation/ubiquitination network of A. nidulans (von Zeska Kress et al., 2012). The protein AN4491 was identified in both, wild type and $\operatorname{csn} E$ deletion strain, whereas protein AN4149 was only co-purified in the deneddylase deficient strain. Both proteins have not been characterized so far. It is possible that the proteins are interaction partners or substrates of the neddylation or ubiquitination machinery. Neither in AN4491 nor in AN4149 conserved domains were identified. AN4491 is a protein of 304 amino acids and is conserved in many ascomycetes, such as in Aspergillus, Neurospora and Penicillium species, as well as Neosartorya fischeri but none of these homologs was studied. AN4149 has a length of 498 amino acids and potential homologs are present in several Aspergilli and other related fungi but with low amino acid conservation.

To analyze if the proteins fulfill developmental functions, deletion strains were constructed, confirmed by Southern hybridization and phenotypically investigated. Both deletion strains resembled the wild type in colony growth and morphology (data not shown). Neither in sexual nor in asexual development the two deletions strains displayed any differences to the wild type. Additionally, the sensitivity against stress-inducing agents was tested. The $\triangle A N 4149$ and the $\triangle A N 4491$ strain grew similar to the wild type on plates containing $2 \%$ ethanol, $10 \mathrm{mM}$ 
hydroxyurea, $0.003 \% \mathrm{H}_{2} \mathrm{O}_{2}, 0.01 \%$ methyl-methanesulfonate, $0.05 \mathrm{mM}$ menadione or $0.006 \%$ SDS, respectively (data not shown).

Taken together, these data suggest that the two NeddH-associated proteins are not required for fungal development under the tested conditions and their cellular functions remain elusive.

\subsection{The sumoylation network in A. nidulans}

\subsubsection{The SumO protein of $A$. nidulans}

Another important member of the family of ubiquitin-like proteins besides $\mathrm{NeddH}$ is SumO. Sumoylation is a conserved process of posttranslational modification that is essential for the viability of higher eukaryotic organisms and S. cerevisiae (Geiss-Friedlander \& Melchior, 2007). In contrast to the neddylation process which is essential in A. nidulans, deletion of the single sumO gene of the fungus is not lethal. Nevertheless, the absence of SumO causes severe defects in development. The $\Delta$ sumO strain is blocked in sexual development at the stage of microcleistothecia formation and asexual development as well as secondary metabolism are impaired (Szewczyk et al., 2008, Wong et al., 2008). To investigate the differences between the essential yeast Smt3p protein, the mammalian Sumo proteins and A. nidulans SumO, the sequences of the five proteins were compared (Figure 15). Yeast Smt3p and SumO share an amino acid sequence homology of $48 \%$ (Figure 15A), but one significant difference between the two proteins is that the Saccharomyces protein carries three lysines (lysine 11, 15 and 19) which are located within sumoylation motifs. Lysines at position 11 and 15 are conserved in Sumo2 and Sumo3 but only lysine 15 is part of a sumoylation motif in the human proteins (Figure 15B red arrow). None of these lysines is conserved in the sequence of SumO. Also not a single sumoylation motif is present in the entire protein sequence (Figure $15)$.

It was shown that the yeast lysines are involved in poly-Smt3 chain formation which is a non essential process (Bylebyl et al., 2003, Tatham et al., 2001). In vivo chain formation of human Sumo proteins is described via the linkage of the marked lysine residue in Sumo2 and 3, whereas Sumo1 is thought to be an endpoint of the polysumoylation reaction (Matic et al., 2008). A. nidulans SumO shares a sequence homology of approximately $40 \%$ to all three human Sumo proteins (Figure 15A). 
A

\begin{tabular}{|l|c|c|c|c|c|}
\hline & SumO & Smt3 & Sumo1 & Sumo2 & Sumo3 \\
\hline SumO & - & $48,0 \%$ & $40,4 \%$ & $42,7 \%$ & $42,7 \%$ \\
\hline Smt3 & $48,0 \%$ & - & $45,1 \%$ & $41,6 \%$ & $41,2 \%$ \\
\hline Sumo1 & $40,4 \%$ & $45,1 \%$ & - & $45,5 \%$ & $44,6 \%$ \\
\hline Sumo2 & $42,7 \%$ & $41,6 \%$ & $45,5 \%$ & - & $95,8 \%$ \\
\hline Sumo3 & $42,7 \%$ & $41,2 \%$ & $44,6 \%$ & $95,8 \%$ & - \\
\hline
\end{tabular}

$\mathbf{B}$

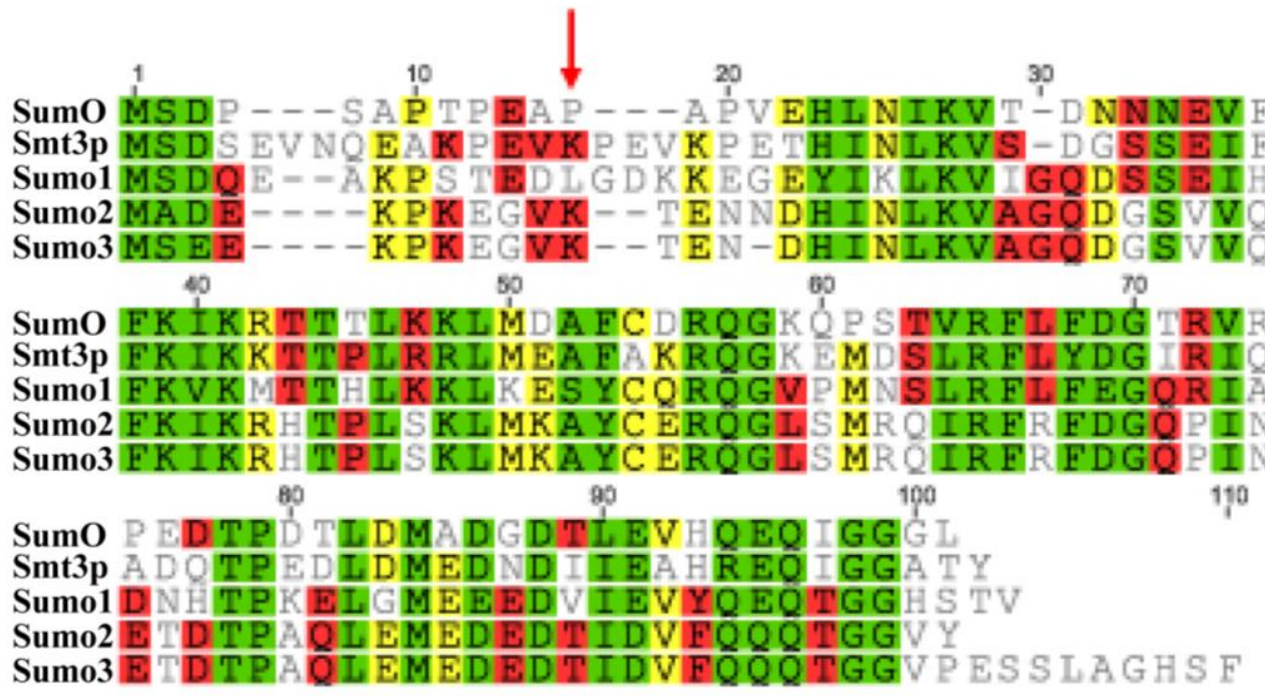

Figure 15: Sequence alignment of SumO, Smt3 and human Sumo1-3.

A Table summarizing the distances of the protein showing the $\%$ of residues that are identical. B Alignment of the protein sequences of A. nidulans SumO, S. cerevisiae Smt3p and human Sumo1-3. Colors indicate the level of similarity. Green: 100\% similar, yellow: 80-100\% similar, red: 60-80\% similar, white: less than $60 \%$ similar. Red arrow marks the lysine residue that is used for chain formation in Sumo2, Sumo3 and Smt3p.

\subsubsection{Deletion of $\operatorname{sumO}$ delays asexual development}

The deletion of Sumo encoding genes is lethal for many organisms, such as mice, S. cerevisiae and A. thaliana (Johnson \& Hochstrasser, 1997, Nacerddine et al., 2005, Saracco et al., 2007). Deletion of sumO in Aspergillus nidulans results in a viable strain with multiple developmental defects (Wong et al., 2008). Incubation of the $\Delta$ sumO mutant produced in our lab in constant white light for $5 \mathrm{~d}$ led to the defects described before and additionally to an increased development of nests as intermediates of sexual development (Laubinger, 2008) (Figure 16A), suggesting that sumoylation is important for the control of light-dependent repression of sexual development in A. nidulans. To further investigate asexual development, the timing of conidiophore formation was observed on oblique thin agar layers. After $20 \mathrm{~h}$ of 
incubation at $37^{\circ} \mathrm{C}$, asexual structures were absent in the $\operatorname{sumO}$ deletion strain but visible in wild type and sumO complementation strain (Figure 16B, top). Further incubation at $37^{\circ} \mathrm{C}$ led to the formation of conidiophores with conidiospores in wild type and $\operatorname{sum} O$ reconstitution strain. In contrast, the $\Delta s u m O$ strain showed reduced and delayed conidiophore formation (Figure 16B, bottom). To determine the conidiophore size, circles surrounding the conidiophore tops were introduced into the pictures (Figure 16B) and their surfaces were calculated. After $28 \mathrm{~h}$ incubation at $37^{\circ} \mathrm{C}$, the conidiophores of the sum $O$ deletion strain displayed a reduction in size to approximately $60 \%$ of wild type conidiophores which was complemented by ectopical reintroduction of sumO driven by its native promoter (Figure 16C).

A

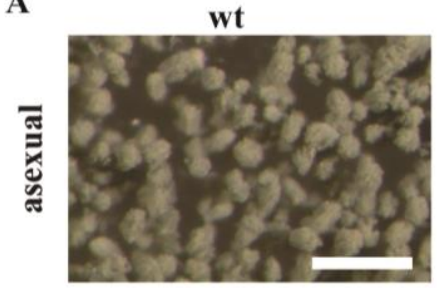

B

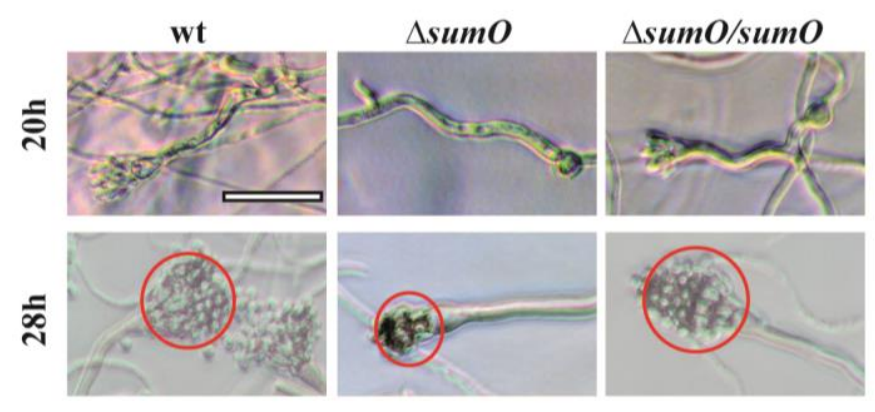

$\Delta s u m O$
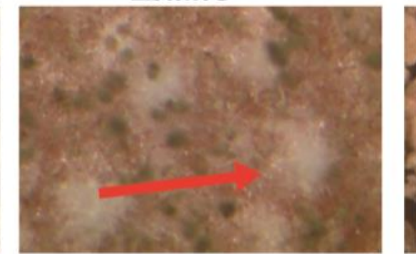

$\Delta \operatorname{sumO} / \mathrm{sumO}$

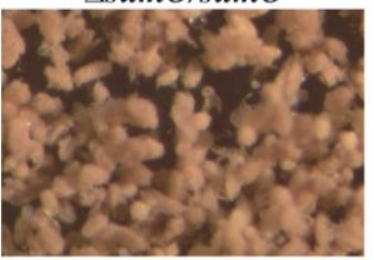

C

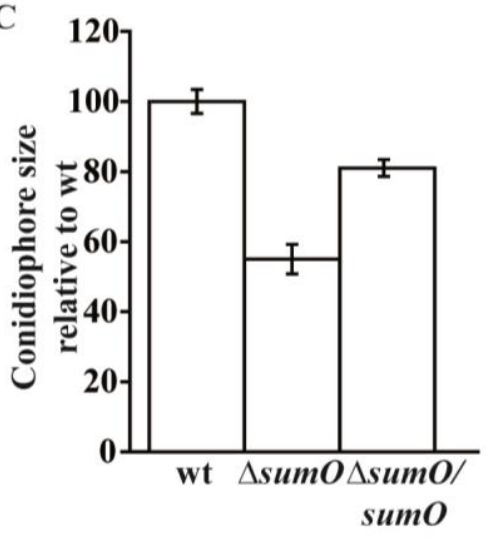

Figure 16: The sumO deletion strain shows defects in light control and multicellular development.

A Asexual development of wild type (wt), the sumO deletion $(\Delta$ sum $O)$ and complementation $(\triangle$ sumO/sumO $)$ strain after 5 days incubation of $1 \times 10^{6}$ spores on $\mathrm{MM}$ at $37^{\circ} \mathrm{C}$ under asexual development inducing conditions. The sum $O$ deletion strain shows nest formation (red arrow), whereas wt and complementation strain only show spores (yellow in complementation strain due to $y A$ mutation). Scale bar $200 \mu \mathrm{m}$. B The sumO deletion strain ( $\Delta$ sumO) displays delayed conidiophore formation. Strains were grown on microscopy slides with oblique thin MM layers for 20 hours or 28 hours at $37^{\circ} \mathrm{C}$. Scale bar $20 \mu \mathrm{m}$. Circles display examples of conidiophore size measurement. C Quantification of conidiophore size. Growth conditions as described in B for 28 hours at $37^{\circ} \mathrm{C}$. Circles were drawn around the upper part of conidiophores and surfaces were calculated using the cellSens software (Olympus). Ratios were calculated relative to the wt in \%. Data are derived from four experiments with 50 conidiophores each (published in (Harting et al., 2013)). 
These data show that the SumO network controls the timing of asexual spore formation, dependent on environmental stimuli, such as light (published in (Harting et al., 2013)).

\subsection{2. $\quad$ SumO isopeptidases}

\subsubsection{The SumO isopeptidase UlpA is required for fungal development}

Deletion of the non essential sumO gene in A. nidulans leads to pleiotrophic developmental defects, indicating the sumoylation of developmental regulators. Sumoylation is a dynamic and reversible process. Therefore, the developmental role of the detachment of SumO from targets was analyzed. Sumo-specific isopeptidases combine two important functions within the Sumo network. First, they catalyze the maturation of the Sumo-precursor. Second, they remove Sumo from the substrate (Wang \& Dasso, 2009, Yeh, 2009).

To investigate the process of desumoylation in A. nidulans, the protein sequences of the two known Smt3-isopeptidases of yeast, Ulp1p and Ulp2p, were used for BLAST searches against the A. nidulans protein database. AN2689 showed approximately $30 \%$ amino acid identity to Ulp1p. In addition the protein has a conserved domain at its C-terminus that contains the catalytic triad Cys-His-Asn which is typical for Ulp proteases, such as UlpA, UlpB and DenA (Figure 17A). According to the similarities and A. nidulans nomenclature, the identified gene was named $u l p A$ and the corresponding protein UlpA.

Ulp1p in yeast is the main processing enzyme of yeast Sumo (Smt3p) (Li \& Hochstrasser, 1999). To analyze the consequences of defects in UlpA, the respective gene was deleted. Correct replacement was confirmed by Southern hybridization. The $\Delta u l p A$ strain displayed a phenotype which resembled the $\operatorname{sumO}$ deletion strain (Figure 17B). The asexual spore production was reduced to approximately $40 \%$ compared to wild type. This is more than the double amount of the spores produced by the sumO deletion strain but still a reduction in comparison to wild type. 
A

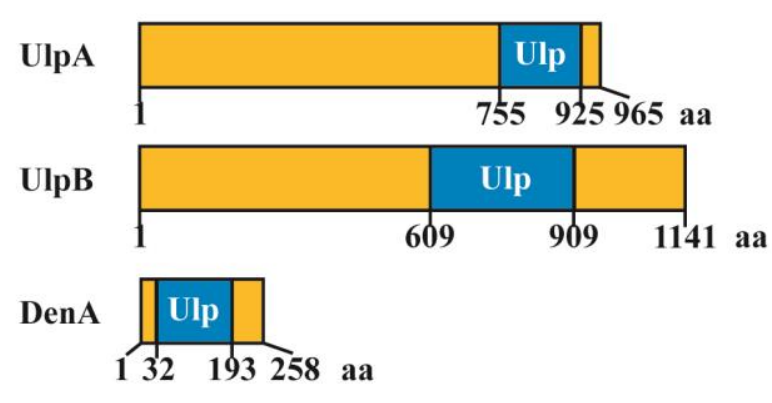

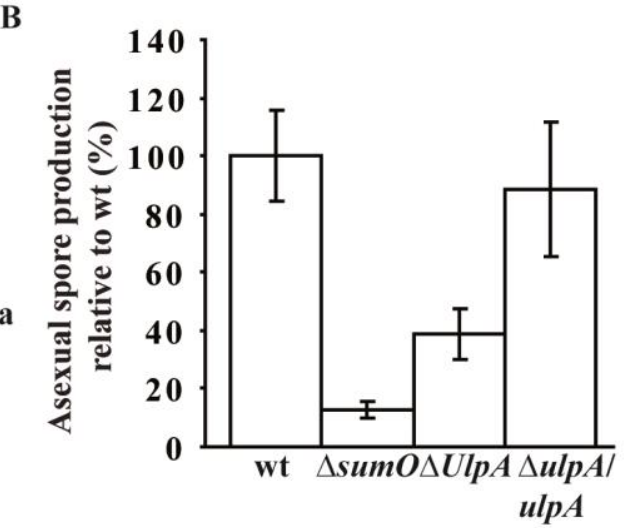

Figure 17: Deletion of $u l p A$ reduces the amount of asexual spores

A Protein domain structure of the proteases UlpA, UlpB and DenA. Ulp marks the Ulp proteinase domain (PS50600) according to the InterProScan results (published in (Harting et al., 2013)). B Quantification of asexual spores produced by the wild type (wt), the sumO $(\triangle \operatorname{sumO})$ and the ulpA $(\triangle u l p A)$ deletion, as well as the $u l p A$ complementation $(\triangle u l p A / u l p A)$ strain, respectively. $1 \times 10^{6}$ spores were plated on solid MM containing appropriate supplements and grown for 3 days at $37^{\circ} \mathrm{C}$. Spore number was determined for five $0.5 \mathrm{~cm}^{2}$ sections per plate. Ratios were calculated related to the wt spore number in \%. Data are derived from four independent experiments.

In addition, the size of the sexual fruit bodies is drastically reduced in comparison to the cleistothecia produced by the wild type (Figure 18). It accounts for around $55 \%$ of the mature wild type cleistothecium (Figure 18A) and is slightly bigger than the fruit bodies produced by the sum $O$ deletion strain but lacks mature ascospores (data not shown). The described phenotypes were complemented upon ectopical reintroduction of $u l p A$ under its native promoter.

The influence of the $u l p A$ deletion on the cellular sumoylation levels was investigated. The whole protein was extracted from mycelium grown under vegetative conditions for $20 \mathrm{~h}$ at $37^{\circ} \mathrm{C}$. Immunoblotting with an antibody specific for A. nidulans SumO revealed a significant change in the overall sumoylation pattern (Figure 18C). The $u l p A$ deletion strain displayed an approximately 25-fold increase in the amount of potentially sumoylated proteins (Figure 18D). Reintroduction of $u l p A$ changed the sumoylation pattern back to the one of the wild type.

These data suggest that UlpA is a SumO-specific isopeptidase which is involved in the removal of SumO from substrates more than in SumO maturation, also the deletion phenotype resembles the one of a SumO deficient strain (published in (Harting et al., 2013)). 


\subsubsection{Crosstalk between the Sumo and NeddH fungal network}

DenA/Den1 is a deneddylase which was first described as a SumO isopeptidase (Christmann et al., 2013, Mukhopadhyay \& Dasso, 2007). In A. nidulans, iIt is a protein of 258 amino acids with a conserved Ulp domain. In order to investigate a potential crosstalk between the processes of (de-)neddylation and (de-)sumoylation in A. nidulans, denA was ectopically expressed under the control of the inducible nitrate promoter in wild type and the $u l p A$ deletion strain. High $\operatorname{den} A$ expression levels were confirmed by qRT-PCR (data not shown).

High levels of DenA had no effect on wild type development and on asexual development of the $u l p A$ deletion strain (data not shown). Analyses of sexual development revealed that the biggest sexual fruit bodies of the strain had an increased size compared to the $\Delta u l p A$ strain (Figure 18A). Whereas the size of the biggest cleistothecia visible in the ulpA deletion strain accounted for around $55 \%$ of the one of the wild type, overexpression of $\operatorname{den} A$ raises the size by around $20 \%$ to approximately $75 \%$ of the size of the wild type cleistothecia (Figure 18A and B). However, these cleistothecia were still defective in the formation of ascospores.

Next, it was tested, whether the strong expression of $\operatorname{den} A$ in the $\Delta u l p A$ background had an impact on the overall cellular sumoylation pattern. Therefore, protein was extracted from cultures, grown under conditions inducing vegetative growth for $20 \mathrm{~h}$ at $37^{\circ} \mathrm{C}$. Similar to the $u l p A$ deletion strain, the strain expressing high levels of $\operatorname{den} A$, showed a strong increase in the amount of potentially sumoylated proteins (Figure 18C and D).

This suggests an effect of high DenA amounts on individual proteins rather than an influence on the global sumoylation pattern in the cell. Alternatively, the increase of sexual fruit body size could be the result of a DenA deneddylation reaction. There seems to be a subtle cellular balance between desumoylation/ deneddylation which might be due to a crosstalk between the NeddH and SumO pathway (published in (Harting et al., 2013)). 
A
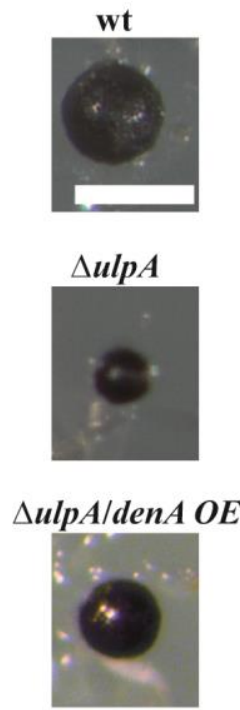

B

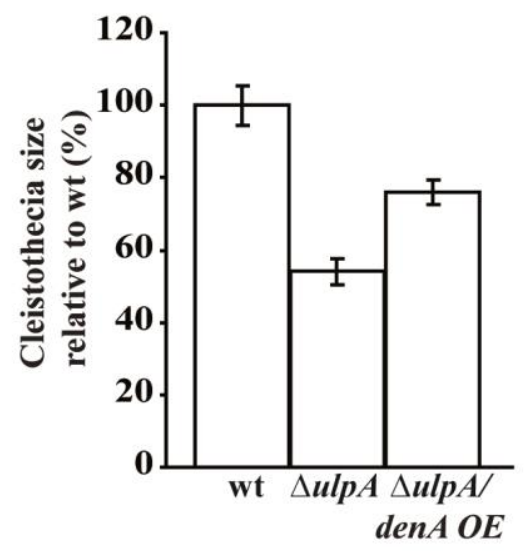

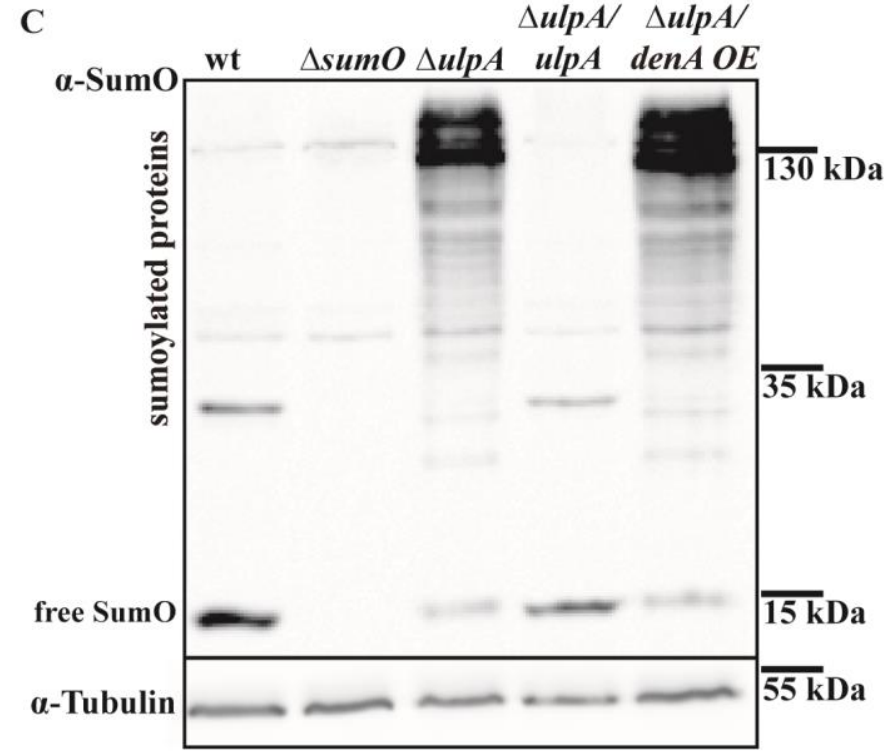

D

Sumoylated proteins

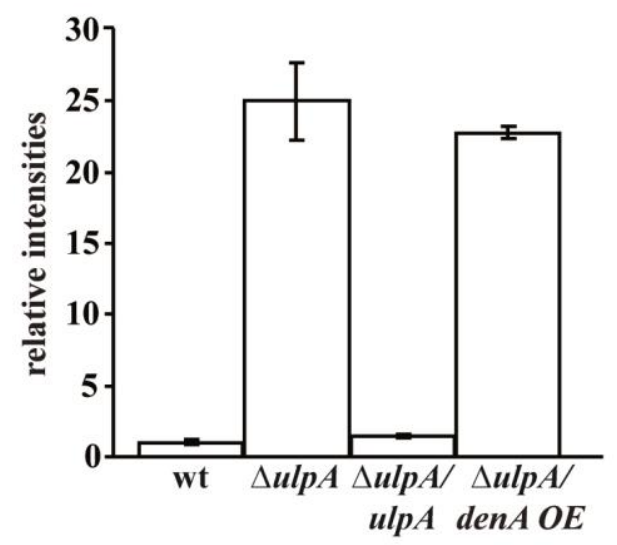

Figure 18: Deletion of ulpA changes the cellular sumoylation pattern.

A Representative sexual fruit bodies of the wild type (wt), the ulpA deletion strain $(\triangle u l p A)$, as well as the $\Delta u l p \mathrm{~A}$ strain, overexpressing DenA ( $\triangle u l p A / d e n A O E$ ) (overexpression promoter inducing conditions). Scale bar $100 \mu \mathrm{m}$. Strains were plated on solid minimal medium containing appropriate supplements and grown for 7 days at $37^{\circ} \mathrm{C}$. B Comparison of the cleistothecia size. Strains were grown as described in A. 25 of the biggest cleistothecia per experiment were measured. Ratios were calculated related to the wild type in \%. Data is derived from four experiments. C Immunoblot of the wild type (wt), the sum $O$ deletion $(\triangle \operatorname{sum} O)$, the ulpA deletion $(\triangle u l p A)$ and complementation strain $(\triangle u l p A / u l p A)$ and the strain, overexpressing DenA in the ulpA deletion background ( $\triangle u l p A / d e n A O E)$ with SumO antibody and tubulin antibody as control. Crude extracts were prepared from 20 hours vegetative grown mycelium. D Quantification of signal intensities using the Fusion-SL7 system with Bio1D software (Peqlab). The relative intensity of the overall amount of sumoylated proteins was put in relation to the one of the wt, whose relative intensity of the sumoylated proteins corresponds to 1 . The signal for tubulin was used as internal standard to normalize signal intensities. Data are derived from two experiments with two replications each (modified published in (Harting et al., 2013)). 


\subsubsection{UlpB is a SumO-processing enzyme}

Ulp2p is the main desumoylating enzyme in yeast, specifically important for cleavage of poly-Sumo (Smt3p) chains (Bylebyl et al., 2003, Li \& Hochstrasser, 2000). A potential homolog for Ulp2p is also present in A. nidulans. AN8192 displays around $35 \%$ amino acid identity to the yeast protein and contains the characteristic Ulp domain. It has a size of 1141 amino acids. According to the similarities and $A$. nidulans nomenclature, the identified gene was named $u l p B$ and the corresponding protein UlpB.

A

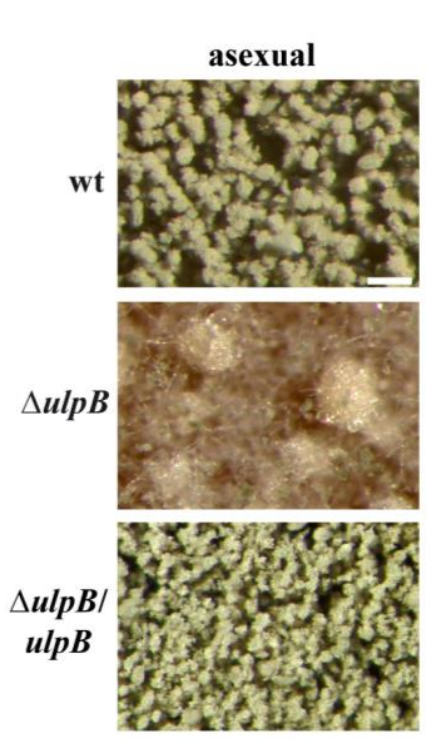

B

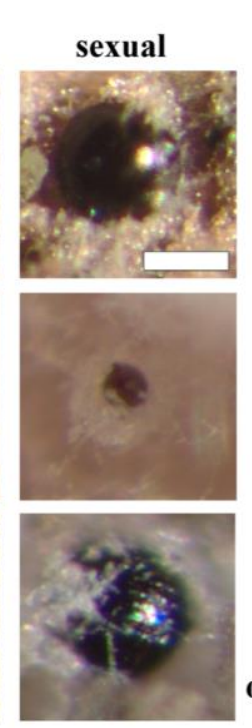

C

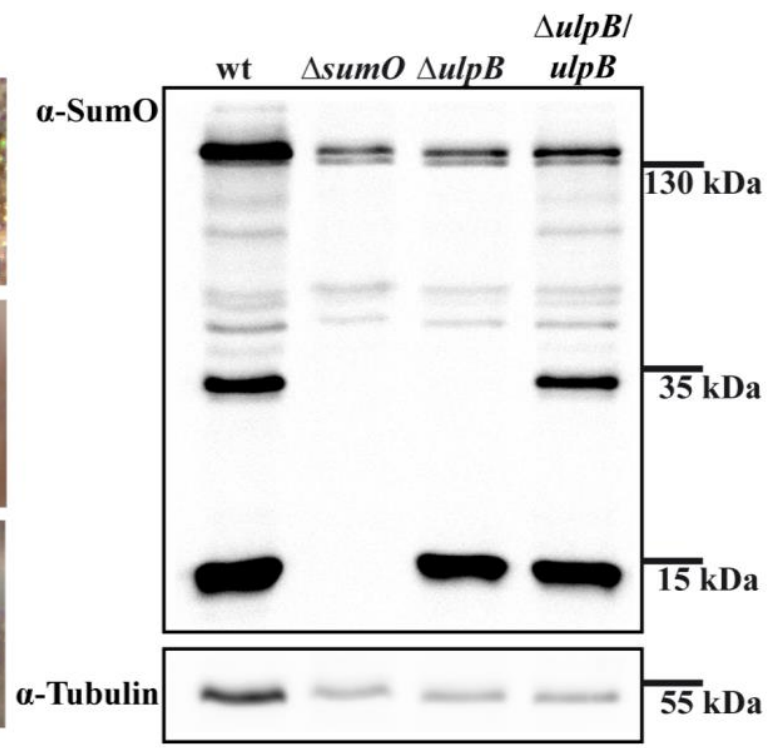

Figure 19: UlpB is a SumO processing enzyme.

A Comparison of asexual development of the wild type (wt), the $u l p B$ deletion $(\triangle u l p B)$ and the $u l p B$ complementation strain $(\triangle u l p B / u l p B) .1 \times 10^{6}$ spores were plated on MM containing appropriate supplements and grown for 5 days at $37^{\circ} \mathrm{C}$. Scale bar $100 \mu \mathrm{m}$ B Comparison of cleistothecia of the wild type (wt), the $u l p B$ deletion $(\triangle u l p B)$ and complementation strain $(\triangle u l p B / u l p B)$. Strains were plated on MM containing appropriate supplements and grown for 7 days at $37^{\circ} \mathrm{C}$ under sexual development inducing conditions. Scale bar $100 \mu \mathrm{m} \mathrm{C} \mathrm{Immunoblot} \mathrm{of} \mathrm{the} \mathrm{wild} \mathrm{type} \mathrm{(wt),} \mathrm{the} \mathrm{sum} O$ $(\triangle \operatorname{sum} O)$ and $u l p B$ deletion $(\triangle u l p B)$ and the $u l p B$ complementation strain $(\triangle u l p B / u l p B)$ with SumO antibody and tubulin antibody as control. Crude extracts were prepared from 20 hours vegetative grown mycelium (published in (Harting et al., 2013)).

Deletion of the $u l p B$ gene resulted in a similar phenotype as the sumO deletion in A. nidulans multicellular development. Induction of asexual development was decreased resulting in the formation of nests and reduced conidiospore amounts 
(Figure 19A). Also the sexual development was blocked at the stage of microcleistothecia (Figure 19B). Western analyses of the cellular sumoylation pattern revealed only free SumO protein but no potentially sumoylated target proteins (Figure 19C). The phenotype was complemented by ectopical expression of $u l p B$ under its native promoter. This suggests that UlpB acts as the processing enzyme for SumO (published in (Harting et al., 2013)).

\subsubsection{Members of the SumO network and the sumoylation pathway}

\subsubsection{Identification of members of the SumO network}

The deletion of sumO has pleiotropic effects on fungal development. However, it is not known which proteins undergo sumoylation in A. nidulans. Western analyses suggested the presence of various sumyolated or SumO-interacting proteins. In order to identify these proteins, a tap::sumO fusion was constructed and introduced at the original sumO locus in a wild type and an $u l p A$ deletion strain. Correct integration into the locus was confirmed by Southern hybridization and expression of the protein was monitored by immunoblotting with a calmodulin binding protein antibody (Figure 20A). Analyses of the phenotype of the strain expressing tap::sumO in the wild type background revealed that the fusion protein is partially functional. Asexual spore production is similar to the one in wild type (Figure 20B). Sexual fruit bodies display an intermediate size compared to the ones of the sumO deletion and the wild type strain (Figure 20C).

The tap::sumO expressing strains as well as their corresponding parental strains were used for purification of SumO network members. Data were collected from three independent experiments with the strain expressing tap::sumO in the wild type background and two independent experiments from the $\Delta u l p A / t a p:: s u m O$ strain. Proteins which were identified in the control experiments were considered as unspecific and are not included in the data set. Candidates for sumoylated or SumOinteracting proteins required at least two identifications in independent experiments according to the parameters mentioned in chapter 2.2.2.6. A list of all candidates including their putative yeast homologs, functions and number of potential sumoylation motifs is given in table 6 . The proteins were categorized according to their annotated functions using the databases and tools described in chapter 2.2.1.2. In general, the amount of identified proteins in the ulpA deficient strain was 
significantly increased, confirming the desumoylation function of the isopeptidase. A total of 56 candidates for sumoylated or sumo-interacting proteins were identified that were arranged into seven categories (Figure 20D).

Only eight out of the 56 proteins did not display any sumoylation motif (Table 6). Homologous proteins for three enzymes involved in the sumoylation pathway were found: the E1-activating enzyme AosA, the E2-conjugating enzyme UbcN, and one potential E3 ligase, SizA. The E1 and E3 enzymes were found in wild type and $u l p A$ deletion background, whereas the E2 enzyme UbcN was only identified in the strain with intact ulpA. The homolog of the second E1-activating enzyme, UbaB, was also identified in the control strains which might be due to unspecific binding or high cellular expression levels.

Additionally, five proteins of the ubiquitination pathway were identified in wild type as well as in the isopeptidase deficient background. We found a homolog of the E1 enzyme for Ubiquitin, the yeast protein Uba1p (AN10266). In addition, four putative subunits of the proteasome were identified (AN4869, AN5784, AN5872 and AN6547), proposed homologs for the yeast proteins Scl1p, Pre7p, Pup2p, Pre5p, respectively. Two out of the four proteasomal subunits do not contain a sumoylation motif, suggesting that these subunits were co-purified because of their interaction with SumO-modified subunits. Alternatively, sumoylated proteins are degraded via the $26 \mathrm{~S}$ proteasome, allowing an enrichment of proteasomal subunits with TAP::SumO. In summary, the identification of proteins from the ubiquitination process suggests a cross-talk of the pathways of sumoylation and ubiquitination.

The largest category of proteins identified has a predicted role in transcription and/or histone modification. In total 27 proteins of this group were co-purified with TAP::SumO. The majority (18 proteins) was identified only in the $u l p A$ deletion background, suggesting that transcriptional regulators are only sumoylated in a small subpopulation in the cell and the sumoylation state becomes stabilized in the isopeptidase deficient strain, allowing identification in our screen (Table 6). A number of proteins were found which have homologs incorporated in the chromatin modifying SAGA (Sppt- $\underline{A} d a-\underline{G} c n 5-\underline{A} c e t y l t r a n s f e r a s e)$ complex, namely the yeast proteins Sgf29p (AN0668), Spt20p (AN0976; RfeE), Taf12p (AN2769), Spt7p (AN4894), Ngg1p (AN0440), Ada2p (AN10763; AdaB) and Gen5p (AN3621; GcnE). The SAGA complex of yeast is an important transcriptional co-activator (Samara \& Wolberger, 2011). 
A

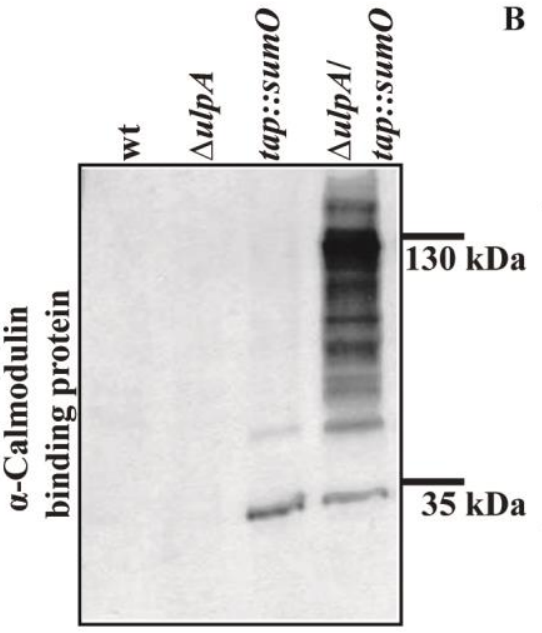

B

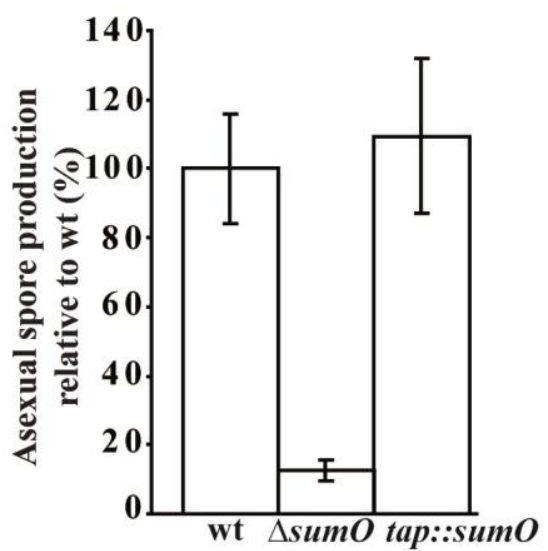

C

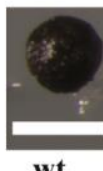

wt

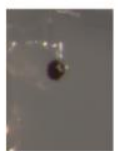

$\Delta \operatorname{sumO}$

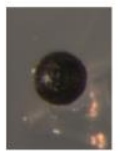

tap::sumO

D

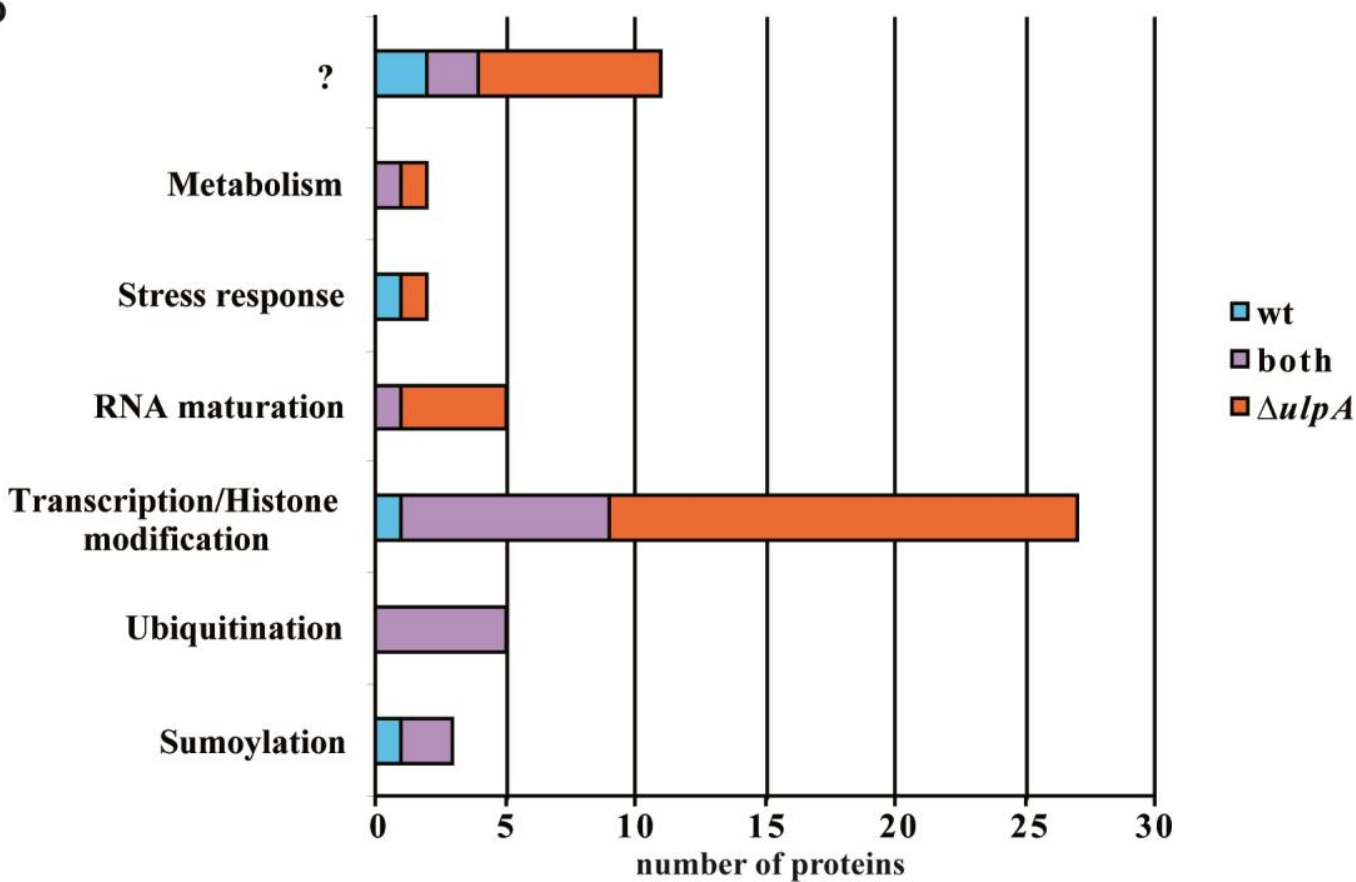

Figure 20: TAP::SumO recruits potentially sumoylated and SumO-interacting proteins.

A Immunoblot of the wild type (wt), the $u l p A$ deletion strain $(\triangle u l p A)$ and the strain expressing tap::sumO in wild type (tap::sumO) and ulpA deletion ( $\triangle$ ulpA/tap::sumO) background with calmodulin binding protein antibody. Crude extracts were prepared from 20 hours vegetative grown mycelium. B Quantification of asexual spores produced by the wild type (wt), the sum $O$ deletion strain $(\triangle$ sumO $)$ and the strain expressing the tap::sumO fusion in wt background (tap::sumO). $1 \times 10^{6}$ spores were plated on $\mathrm{MM}$ containing appropriate supplements and grown for 3 days at $37^{\circ} \mathrm{C}$. Spore number was determined for five $0.5 \mathrm{~cm}^{2}$ sections per plate. Ratios were calculated related to the wt spore number in \%. Data are derived from four experiments. C Representative sexual fruit bodies of the wild type (wt) and the $\operatorname{sum} O$ deletion strain $(\triangle \operatorname{sumO})$ as well as the strain expressing the tap::sumO fusion protein (tap::sumO). Scale bar $100 \mu \mathrm{m}$. D Graphical overview of the different categories and the number of proteins identified in wild type (wt), ulpA deletion ( $\triangle u l p A)$, or ulpA deletion and wild type background (both), respectively. "?" marks proteins of unknown function (published in (Harting et al., 2013)). 
In A. nidulans, not much about these proteins is known. RfeE was found to be up-regulated upon DNA damage stress (Malavazi et al., 2006) and AdaB and GcnE were connected to nucleosome positioning (Reyes-Dominguez et al., 2008). Additionally, subunits of transcription factor complexes were found, such as Toa2p (AN2181), Tfa2p (AN5498) and Taf1p (AN3907). Several other proteins involved in histone modification were co-purified, such as AN1060, the homolog of yeast Rph1p, a histone demethylase targeting trimethylated lysine 36 in histone 3 (Tu et al., 2007). Another example is the homolog of Sin3p (AN1546), a histone deacetylase involved in transcriptional repression but also positive regulation of gene expression (Kadosh \& Struhl, 1998, Silverstein \& Ekwall, 2005, Yoshimoto et al., 1992). In addition, one subunit of the COMPASS complex, CclA (AN9399) (Bok et al., 2009) was found. The COMPASS complex is responsible for methylation of histone $\mathrm{H} 3$ at lysine 4 that is involved in transcriptional regulation (Wood et al., 2005). Several subunits of the RNA polymerase were identified. Interestingly, also an important regulator of development in A.nidulans, the WD repeat protein RcoA was co-purified with TAP::SumO. The $r c o A$ deletion strain displayed reduced growth rates, a decreased asexual spore production, an altered secondary metabolism and a block in sexual development (Hicks et al., 2001, Todd et al., 2006). RcoA is the homolog of the yeast protein Tup1p which acts together with Cyc8p as corepressor for many genes (Smith \& Johnson, 2000). Also the homolog of Cyc8p, SsnF (AN12054) was identified in our screen (Table 6).

Table 6: Proteins co-purified with TAP-SumO.

\begin{tabular}{|c|c|c|c|}
\hline $\begin{array}{c}\text { AN number } \\
\text { (name) }\end{array}$ & $\begin{array}{c}\text { Yeast } \\
\text { homolog } \\
\text { (putative) }\end{array}$ & $\begin{array}{c}\text { Function (putative) } \\
\text { according to homology, } \\
\text { domains and literature }\end{array}$ & $\begin{array}{c}\text { SumO sites } \\
\text { (predicted) }\end{array}$ \\
\hline \multicolumn{5}{|c|}{ Sumoylation } \\
\hline 2298 & Aos1p & SumO E1 activation enzyme & 1 \\
\hline 4399 & Ubc9p & SumO E2 conjugation enzyme & $/$ \\
\hline 10822 & Siz1p & SumO E3 ligase & 4 \\
\hline \multicolumn{5}{|c|}{ Ubiquitination } \\
\hline 4869 & Scl1p & 20S proteasome subunit & 1 \\
\hline 5784 & Pre7p & 20S proteasome subunit & $/$ \\
\hline 5872 & Pup2p & 20S proteasome subunit & 1 \\
\hline 6547 & Pre5p & 20S proteasome subunit & $/$ \\
\hline 10266 & Uba1p & Ubiquitin E1 activation enzyme & 4 \\
\hline \multicolumn{5}{|c|}{ Transcription/Histone modification } \\
\hline 0253 & Top1p & $\begin{array}{c}\text { DNA Topoisomerase I } \\
\text { (Van Dross et al., 1997) }\end{array}$ \\
\hline (Top1) & \multicolumn{5}{|c}{} \\
\hline
\end{tabular}


Table 6: Proteins co-purified with TAP-SumO, continued.

\begin{tabular}{|c|c|c|c|}
\hline $\begin{array}{l}\text { AN number } \\
\quad \text { (name) }\end{array}$ & $\begin{array}{c}\text { Yeast } \\
\text { homolog } \\
\text { (putative) }\end{array}$ & $\begin{array}{l}\text { Function (putative) } \\
\text { according to homology, } \\
\text { domains and literature }\end{array}$ & $\begin{array}{l}\text { SumO sites } \\
\text { (predicted) }\end{array}$ \\
\hline 0440 & Ngg1p & $\begin{array}{c}\text { Histone acetyltransferase; } \\
\text { SAGA, SLIK, ADA complexes }\end{array}$ & 4 \\
\hline 0668 & Sgf29p & $\begin{array}{c}\text { Factor involved in } \\
\text { transcriptional regulation } \\
\text { through SAGA }\end{array}$ & 2 \\
\hline 0671 & & bZIP transcription factor & 2 \\
\hline $\begin{array}{l}0976 \\
(\mathrm{RfeE})\end{array}$ & Spt20p & Subunit of SAGA complex & 5 \\
\hline 1060 & Rph1p & Histone demethylase & 6 \\
\hline 1546 & $\operatorname{Sin} 3 p$ & $\begin{array}{l}\text { Histone deacetylase complex } \\
\text { subunit }\end{array}$ & 6 \\
\hline $\begin{array}{c}2141 \\
(\mathrm{NimU})\end{array}$ & & $\begin{array}{l}\text { Essential protein required for } \\
\text { progression through mitosis } \\
\text { (Pitt et al., 2004) }\end{array}$ & 1 \\
\hline 2181 & Toa2p & TFIIA subunit & / \\
\hline 2415 & Rpc40p & RNA polymerase subunit & / \\
\hline 2769 & Taf12p & $\begin{array}{l}\text { Subunit of TFIID and SAGA } \\
\text { complexes }\end{array}$ & 1 \\
\hline $\begin{array}{c}3621 \\
(\mathrm{GcnE})\end{array}$ & Gen5p & $\begin{array}{c}\text { Subunit of histone } \\
\text { acetyltransferase SAGA } \\
\text { complex } \\
\text { (Reyes-Dominguez et al., 2008) }\end{array}$ & 1 \\
\hline $\begin{array}{l}3688 \\
(\text { SrrA })\end{array}$ & Skn7p & $\begin{array}{c}\text { Response regulator } \\
\text { (Hagiwara et al., 2007) }\end{array}$ & / \\
\hline 3907 & Taf1p & Subunit of TFIID & 2 \\
\hline 4445 & Swc4p & $\begin{array}{c}\text { Histone exchange factor and } \\
\text { subunit of histone } \\
\text { acetyltransferase complex }\end{array}$ & 1 \\
\hline 4894 & Spt7p & $\begin{array}{l}\text { Subunit of SAGA complex (also } \\
\text { SLIK complex) }\end{array}$ & 7 \\
\hline 5498 & Tfa2p & Subunit of TFIIE & 3 \\
\hline $\begin{array}{c}6505 \\
(\mathrm{RcoA})\end{array}$ & Tup1p & $\begin{array}{l}\text { WD40 repeat protein, required } \\
\text { for sex. development } \\
\text { (Hicks et al., 2001, Todd et al., } \\
\text { 2006) }\end{array}$ & 1 \\
\hline 7170 & & Transcription factor & 1 \\
\hline 7608 & & Subunit of RNA polymerase & 3 \\
\hline $\begin{array}{c}9399 \\
(\mathrm{CclA})\end{array}$ & Bre2p & $\begin{array}{c}\text { Subunit of COMPASS complex, } \\
\text { histone methylation } \\
\text { (Bok et al., 2009) }\end{array}$ & 1 \\
\hline $\begin{array}{c}9538 \\
\text { (new: } 12489)\end{array}$ & & Transcription factor & 1 \\
\hline 10316 & Rpo31p & Subunit of RNA polymerase & 5 \\
\hline
\end{tabular}


Table 6: Proteins co-purified with TAP-SumO, continued.

\begin{tabular}{|c|c|c|c|}
\hline $\begin{array}{l}\text { AN number } \\
\text { (name) }\end{array}$ & $\begin{array}{c}\begin{array}{c}\text { Yeast } \\
\text { homolog } \\
\text { (putative) }\end{array} \\
\end{array}$ & $\begin{array}{l}\text { Function (putative) } \\
\text { according to homology, } \\
\text { domains and literature } \\
\end{array}$ & $\begin{array}{l}\text { SumO sites } \\
\text { (predicted) }\end{array}$ \\
\hline $\begin{array}{c}10763 \\
(\text { AdaB })\end{array}$ & Ada2p & $\begin{array}{c}\text { Subunit of histone } \\
\text { acetyltransferase (Reyes- } \\
\text { Dominguez et al., 2008) }\end{array}$ & / \\
\hline 10855 & Pol12p & $\begin{array}{c}\text { Subunit of DNA polymerase } \\
\text { alpha-primase complex }\end{array}$ & 3 \\
\hline 11125 & Grs1p & Transcription termination factor & 1 \\
\hline $\begin{array}{l}12054 \\
(\mathrm{SsnF})\end{array}$ & Сус8p & $\begin{array}{l}\text { Transcriptional co-repressor } \\
\text { (Garcia et al., 2008) }\end{array}$ & 2 \\
\hline \multicolumn{4}{|c|}{ RNA maturation } \\
\hline 0161 & Clp1p & $\begin{array}{l}\text { Subunit of cleavage and } \\
\text { polyadenylation factor I }\end{array}$ & 1 \\
\hline 1635 & Prp39p & snRNP protein & 1 \\
\hline 3082 & Cft2p & $\begin{array}{l}\text { Subunit of cleavage and } \\
\text { polyadenylation factor }\end{array}$ & 1 \\
\hline 5124 & Pcf11p & $\begin{array}{l}\text { Subunit of cleavage and } \\
\text { polyadenylation factor }\end{array}$ & 1 \\
\hline 10574 & $\operatorname{Prp} 24 p$ & Splicing factor & 1 \\
\hline \multicolumn{4}{|c|}{ Stress response } \\
\hline $\begin{array}{c}0241 \\
(\text { SodA) }\end{array}$ & Sod1p & $\begin{array}{c}\text { Superoxide dismutase } \\
\text { (Oberegger et al., 2000) }\end{array}$ & 1 \\
\hline 10978 & Ntg1p & DNA repair & 10 \\
\hline \multicolumn{4}{|c|}{ Metabolism } \\
\hline $\begin{array}{c}0554 \\
(\text { AldA) }\end{array}$ & Ald $4 p$ & $\begin{array}{l}\text { Aldehyde dehydrogenase } \\
\text { (Pickett et al., 1987) }\end{array}$ & 3 \\
\hline 4258 & Ura6p & Uridylate kinase & 1 \\
\hline \multicolumn{4}{|c|}{ Unknown function } \\
\hline 1466 & & Unknown & 8 \\
\hline 3038 & & Unknown & 2 \\
\hline 4622 & & Unknown & 1 \\
\hline 4705 & & Unknown & 2 \\
\hline 5212 & & Unknown & 3 \\
\hline 5532 & & Unknown & 3 \\
\hline 6560 & & Unknown & 1 \\
\hline $\begin{array}{c}9087 \\
\text { (OefA) }\end{array}$ & & $\begin{array}{c}\text { LisH domain containing protein, } \\
\text { deletion strains show fluffy } \\
\text { phenotype } \\
\text { (Lee et al., 2005) }\end{array}$ & 1 \\
\hline 10317 & & Unknown & 3 \\
\hline 10785 & & Unknown & 1 \\
\hline 10799 & & Unknown & 1 \\
\hline 11119 & & Unknown & 1 \\
\hline
\end{tabular}

blue: proteins co-purified with TAP-SumO in wt background, purple: proteins copurified with TAP-SumO in wt and $\triangle u l p A$ background, orange: proteins co-purified with TAP-SumO in $\triangle u l p A$ background (modified published in (Harting et al., 2013)) 
Additionally, five proteins were identified, whose homologs play a role in RNA maturation (AN0161, AN1635, AN3082, AN5124, AN10574), such as subunits of cleavage and polyadenylation factors. Most of the proteins were only identified in the $u l p A$ deletion strain. Two proteins were co-purified that are involved in stress response, the superoxide dismutase SodA (AN0241) (Oberegger et al., 2000) and the homolog of Ntg1p of yeast (AN10978), a protein important for the response to oxidative stress (Alseth et al., 1999). Two proteins were identified as potential substrates which are involved in metabolic processes, the aldehyde dehydrogenase AldA (AN0554) (Pickett et al., 1987) and the homolog of the yeast uridylate kinase Ura6p (AN4258) (Table 6). Both proteins contain potential sumoylation sites and might reflect an influence of sumoylation on metabolic processes.

These data support a specific role of sumoylation and desumoylation on gene expression during fungal development which might explain the pleiotropic phenotypes of the sumO deletion strain. The large group of additionally enriched proteins in the strain deficient in the SumO isopeptidase UlpA compared to wild type corroborates a highly dynamic turnover between sumoylated and desumoylated target proteins required for correct multicellular differentiation in fungi (published in (Harting et al., 2013)).

\subsubsection{The E1 activation and E2 conjugation enzymes of the SumO pathway are required for multicellular development in A. nidulans}

As described above, potential homologs of enzymes involved in the sumoylation pathway were co-purified with TAP::SumO. The role of the enzymes, catalyzing the individual steps of the sumoylation reaction, for fungal development was analyzed. The Sumo activating enzyme is formed by a heterodimer of Aos1 and Uba2 in S. cerevisiae (Johnson et al., 1997). The Aspergillus homologues AN2298 and AN2450 show an amino acid identity of around 30\% to $40 \%$ compared to the yeast proteins. Both proteins have domains, typical for activation enzymes of ubiquitin-like proteins. According to the similarities and A.nidulans nomenclature, the identified genes were named $a o s A$ and $u b a B$ and the corresponding proteins Aos A and UbaB. Additionally, the homologue of the E2 enzyme Ubc9 was identified. AN4399 displays an amino acid identity of approximately 60\% compared to the yeast protein and a similar domain structure. Therefore, the gene was named $u b c N$ and the corresponding protein $\mathrm{UbcN}$. Furthermore, a protein with approximately 
$30 \%$ amino acid identity to the yeast E3 ligase Siz1 was co-purified. AN10822 has a DNA-binding domain (InterProScan signature PF02037), a zinc finger motif (PF02891) and a PINIT domain (PF14324) which is characteristic for Siz/PIAS Sumo E3 ligases. According to the similarities and A. nidulans nomenclature, the identified gene was named sizA and the corresponding protein SizA. Deletion strains for the four genes were constructed and confirmed by Southern hybridization. The sumoylation pattern was analyzed for differences to the wild type by immunoblotting with a SumO-specific antibody. In the $a o s A, u b a B$ and $u b c N$ deletion strain the signal of the free cellular SumO was present, while higher signals of potentially sumoylated proteins were not longer detectable (Figure 21A). In the $\Delta$ sizA strain, the signal of the protein migrating between 25 and $35 \mathrm{kDa}$ was still detectable, although the signal intensity decreased. The signal in the wild type strain was approximately three times stronger than in the sizA deletion strain (Figure 21B). As internal standard, the membrane was reprobed with a tubulin antibody.

A

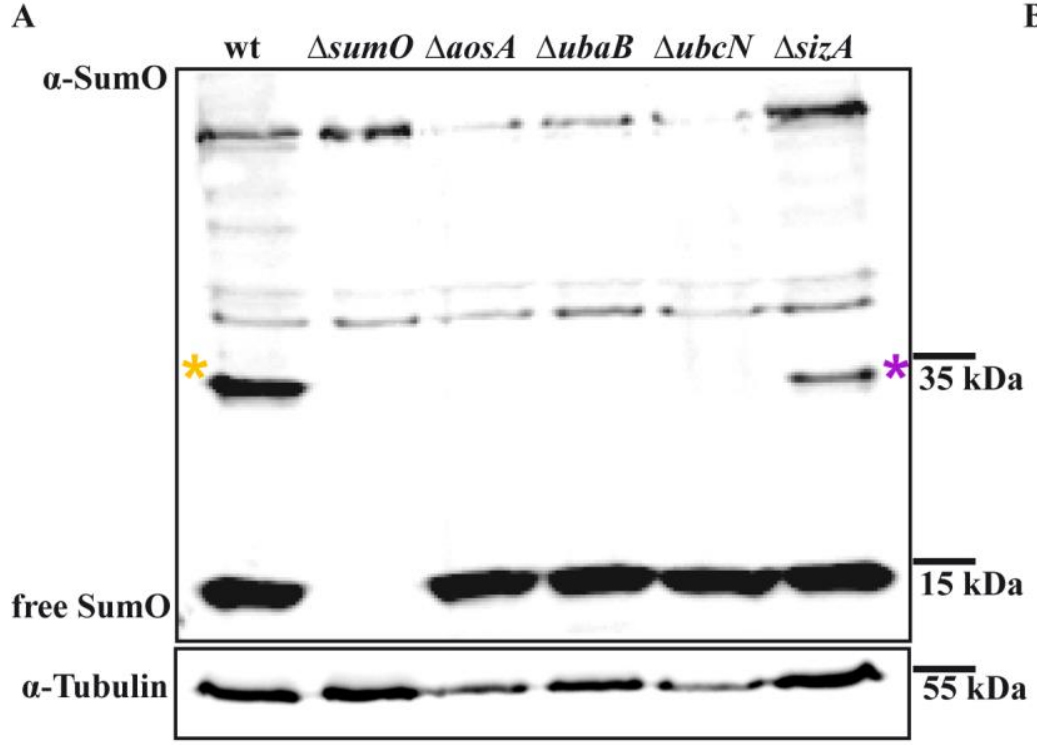

B

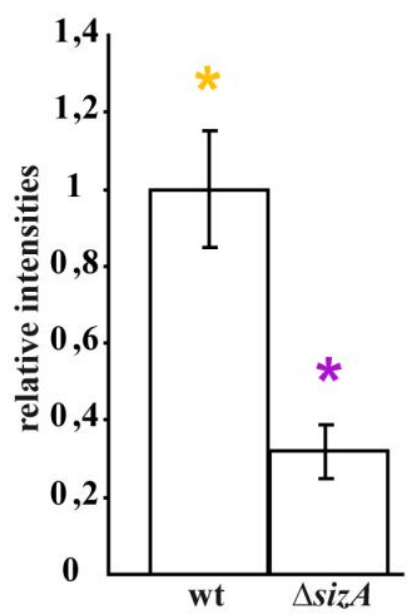

Figure 21: Deletions of $a o s A, u b a B, u b c N$ and $s i z A$ change the cellular sumoylation pattern.

A Immunoblot of the wild type (wt), and the deletion strains of sumO $(\Delta \operatorname{sum} O)$, aosA $(\triangle a o s A), u b a B$ $(\triangle u b a B), u b c N(\triangle u b c N)$ and $s i z A(\triangle s i z A)$ with SumO antibody and tubulin antibody as control. Crude extracts were prepared from 20 hours vegetative grown mycelium. * indicates a certain type of sumoylated protein/SumO interacting protein of the size of 25-35 kDa. B Quantification of signal intensities. The relative intensity of band * of the sizA deletion strain $(\triangle s i z A)$ was put in relation to the wt which relative intensity corresponds to 1 . The signal for tubulin was used as internal standard to normalize signal intensities. Data are derived from two independent experiments with four replications each (published in (Harting et al., 2013)). 
Additionally to the molecular consequences, the developmental phenotype of the deletion strains was investigated. The deletion strains of $a o s A, u b a B$ and $u b c N$ resembled the sumO deletion strain. In sexual development, fruit body formation was blocked at the stage of microcleistothecia (Figure 22A). Under asexual inducing conditions, the deletion strains displayed a reduction in asexual spore production and an elevated induction of sexual fruit body formation (Figure 22B). In addition, the colony morphology of the strains was disturbed. While the wild type and the $\Delta$ sumO strain showed an evenly colony shape when point-inoculated on minimal medium, the deletion strains of $a o s A, u b a B$ and $u b c N$ displayed a ragged, uneven form (Figure 22A). The bottom view of the colonies revealed that all three deletion strains produced a brown-reddish color different from the dye produced of the sumO deletion strain.

A
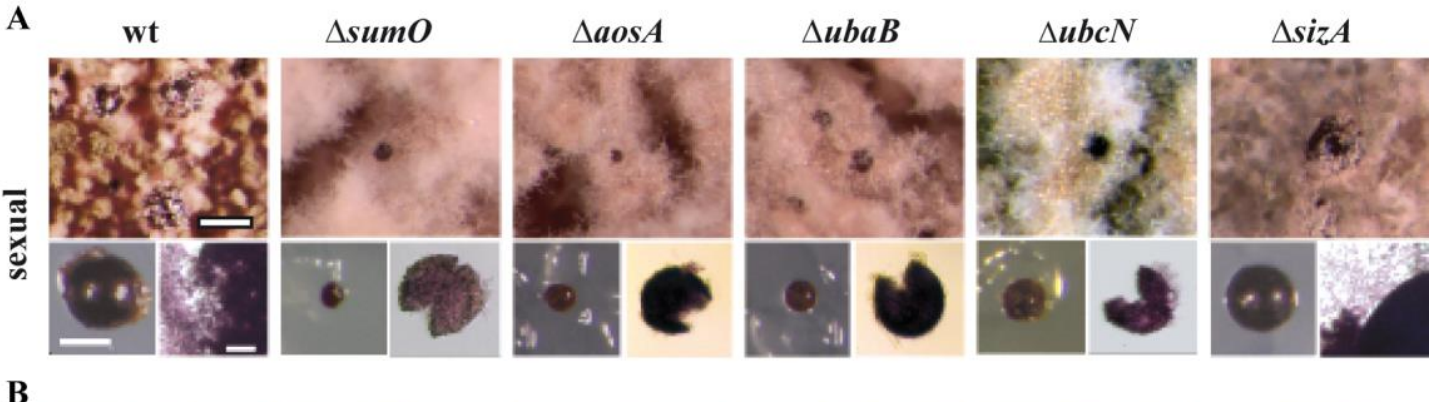

B
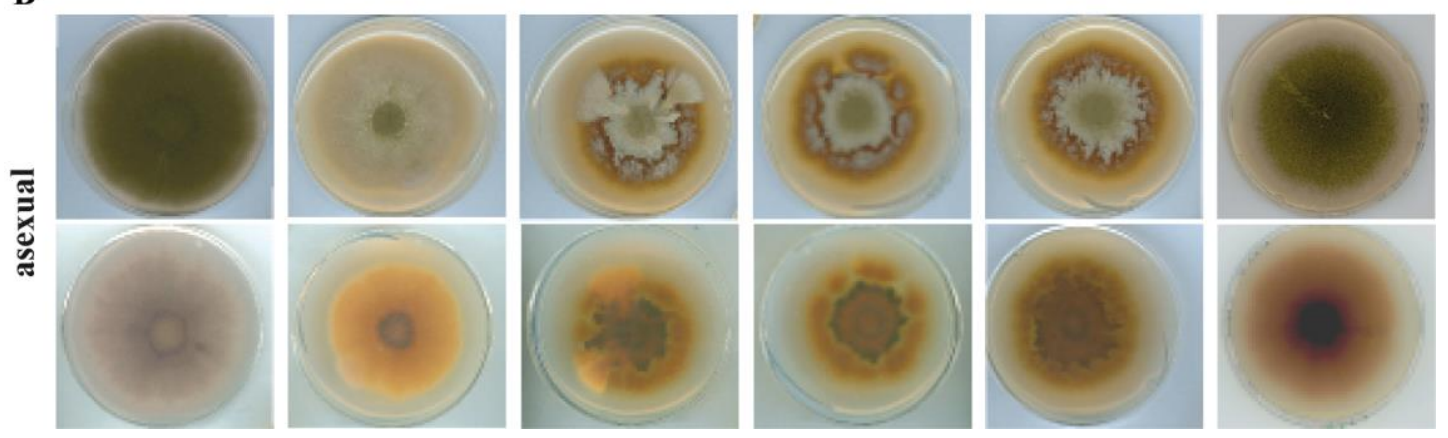

Figure 22: Deletions of $a o s A, u b a B$ and $u b c N$ affect asexual and sexual development.

A Sexual development of the wild type (wt), the deletion strains of $\operatorname{sumO}(\triangle \operatorname{sumO}), \operatorname{aos} A(\triangle \operatorname{aos} A)$, $u b a B(\triangle u b a B), u b c N(\triangle u b c N)$ and $\operatorname{sizA}(\Delta s i z A)$. Upper panel: sexual fruit bodies on plate (Scale bar $100 \mu \mathrm{m}$ ), lower panel: close up view of cleistothecia (left, scale bar $100 \mu \mathrm{m}$ ) and squeezed fruit bodies under the microscope (right, scale bar $20 \mu \mathrm{m}$ ). Strains were grown on solid minimal medium containing appropriate supplements for 7 days at $37^{\circ} \mathrm{C}$ under sexual development inducing conditions. B Colony view of the wild type (wt), the deletion strains of $\operatorname{sumO}(\triangle \operatorname{sumO}), \operatorname{aos} A(\triangle \operatorname{aos} A), u b a B$ $(\triangle u b a B), u b c N(\triangle u b c N)$ and sizA $(\triangle \operatorname{sizA}) .500$ spores of each strain were point-inoculated on solid minimal medium containing appropriate supplements and grown for 5 days at $37^{\circ} \mathrm{C}$ under asexual development inducing conditions. Top (upper panel) and bottom (lower panel) of the plates are shown (published in (Harting et al., 2013)). 
The observed phenotypes were complemented by reintroduction of the genes at the original loci. The double deletion strain of aosA and $u b a B$ showed the phenotype of the single deletion strains (data not shown). In contrast, the sizA deletion strain developed normal sized cleistothecia with ascospores and a regular colony shape. However, there is an increase in sexual nest formation in comparison to wild type during cultivation conditions in light which favors asexual development and repress sexual development.

Taken together, our data suggest that SizA contributes to fungal development less than the E1 and E2 enzymes. This indicates the presence of additional Sumo E3 ligases which are encoded in the genome of A. nidulans. These E3 ligases might have redundant or further roles in development, whereas SizA might play a specific role in the formation of nests (published in (Harting et al., 2013)).

\subsubsection{The COMPASS complex controls development of Aspergillus nidulans}

\subsubsection{SetA is part of an $A$. nidulans COMPASS complex}

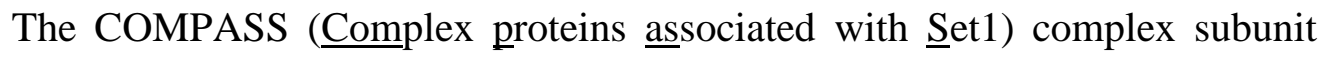
CclA was identified as part of the fungal sumoylation network. A cclA deletion strain displayed a reduction in growth, as well as an altered colony morphology and secondary metabolism (Bok et al., 2009, Giles et al., 2011). The COMPASS complex is a histone methyltransferase, important for the regulation of gene expression connected to the methylation of lysine 4 at histone 3 (Eissenberg \& Shilatifard, 2010). In A. nidulans, all subunits of the COMPASS complex are conserved. In this study, the core subunit of the complex, which is Set1p in yeast, was investigated. In A. nidulans, AN5795 encodes a protein of 1220 amino acids sharing rather low amino acid identity to S. cerevisiae Set1 in the C-terminal region, whereas the $\mathrm{N}$-terminal region is more conserved (approximately 50\% identity). At their N-termini the two proteins have a conserved SET domain (InterProScan signature PF00856). At its C-terminus, the A. nidulans protein carries an RNA recognition motif (PF00076). According to the similarities and A.nidulans nomenclature, the identified gene was named setA and the corresponding protein SetA. SetA carries seven putative sumoylation motifs with the lysines K344, K370, K380, K531, K544, K915 and K1195 as putatively modified. The motifs including 
lysine 370 and 1195 are reversed sumoylation motifs. Analyses with the yeast SetA counterpart revealed a biochemical interaction with yeast Sumo (Smt3p) (Wohlschlegel et al., 2004). Another protein containing a Set domain, PR-Set7, was shown to be sumoylated in human (Spektor et al., 2011).

The setA locus was replaced by a setA::tap fusion to verify the interactions within the COMPASS complex. Correct integration in the locus was confirmed by Southern hybridization. The fusion protein was partially but not fully functional with respect to the developmental phenotype (data not shown). However, the fusion protein was not detectable in western hybridizations with calmodulin binding protein antibody which might be due to low expression levels of the protein (data not shown; see also chapter 3.2.4.4.). Nevertheless, purification was performed with mycelium grown under vegetative conditions for $20 \mathrm{~h}$ at $37^{\circ} \mathrm{C}$. Six proteins were co-purified with SetA which are potential homologs of COMPASS complex subunits (Table 7) (Roguev et al., 2001) and which were not identified in a wild type strain, used as control.

Table 7: Proteins co-purified with SetA-TAP.

\begin{tabular}{|c|c|c|}
\hline $\begin{array}{c}\text { AN number } \\
\text { (name) }\end{array}$ & $\begin{array}{c}\text { Yeast homolog } \\
\text { (putative) }\end{array}$ & Peptides identified \\
\hline 0065 & Swd2p & K.DDTVSIWDLGSR.Y \\
\hline 0179 & Shg1p & $\begin{array}{l}\text { R.TDEEYALLVK.A } \\
\text { K.AATLIEGAVDR.S } \\
\text { K.GGFDHIR.K }\end{array}$ \\
\hline 0808 & Swd1p & $\begin{array}{l}\text { R.AAPSKDEEIDPAVAAK.Q } \\
\text { R.YLLSSSQDWK.C } \\
\text { R.QIQSLSWSR.D }\end{array}$ \\
\hline 2850 & Spp1p & $\begin{array}{l}\text { K.VQQQDVAFEER.T } \\
\text { R.VDQEDAVGAAAER.F } \\
\text { K.QSSASLANSPAPEPK.K } \\
\text { K.SKLDNQDNESVSSR.R } \\
\text { R.NTFLTLVR.H } \\
\text { K.IYYCGIIDGK.N } \\
\text { R.YLNPEQVEVM*K.Q } \\
\text { R.IPEDDPFYQR.S } \\
\text { R.TLESDLAECEK.E }\end{array}$ \\
\hline 3926 & Swd3p & $\begin{array}{l}\text { R.FSPDASM*IASGGADGAVK.V } \\
\text { R.IWDTSTGQCLR.T } \\
\text { R.TLVHEDNPPVSSVK.F }\end{array}$ \\
\hline $\begin{array}{c}9399 \\
(\mathrm{CclA}) \\
(\text { Bok } \text { et } \text { al. }, 2009)\end{array}$ & Bre2p & $\begin{array}{l}\text { R.FSPAPPSTFSNQGDGVR.S } \\
\text { K.DALAVTTNEPWHTAR.A } \\
\text { R.DYAFNLK.E } \\
\text { R.TLPGSSITIYK.N } \\
\text { K.ELYAFLPPASR.L }\end{array}$ \\
\hline
\end{tabular}


Homologs for the yeast proteins Swd2p (AN0065), Shg1p (AN0179), Swd1p (AN0808), Spp1p (AN2850), Swd3p (AN3926) and Bre2p (CclA, AN9399) were found. A single subunit, the homolog of S. cerevisiae Sdc1p, was missing, although with AN6677 A. nidulans exhibits a protein that shares great similarity with the yeast subunit.

Taken together, these data suggest that a COMPASS complex as described in other organisms is formed in A. nidulans (published in (Harting et al., 2013)).

\subsubsection{SetA is required for sexual development}

The function of the COMPASS complex for the development of A. nidulans was addressed genetically. To analyze the function of SetA in fungal development, the coding sequence of the gene was replaced by a deletion cassette. After verification of correct integration by Southern hybridization, sexual development of the setA deletion strain compared with the wild type and the complementation strain was investigated.

The colony morphology appeared to be dramatically disturbed under sexual growth conditions (Figure 23A). Also secondary metabolism was affected as a dark brown-red pigment could be observed. The wild type and the complementation strain formed mature cleistothecia, surrounded by Hülle cells, whereas sexual fruit body formation of the $\Delta s e t A$ strain was blocked at an early stage of development (Figure 23B). Microscopic analyses of the nests produced by the setA deletion strain revealed the formation of Hülle cells but no fruit body precursor could be detected. Further incubation up to $15 \mathrm{~d}$ resulted in the formation of fruit body prcursors in individual nests but never in mature cleistothecia with ascospores. In contrast, the complementation strain showed wild type like sexual development and cleistothecia formation (Figure 23).

These results show that the COMPASS complex subunit SetA is involved in sexual development, as fruit body formation is completely blocked upon deletion of the corresponding gene (published in (Harting et al., 2013)). 
A

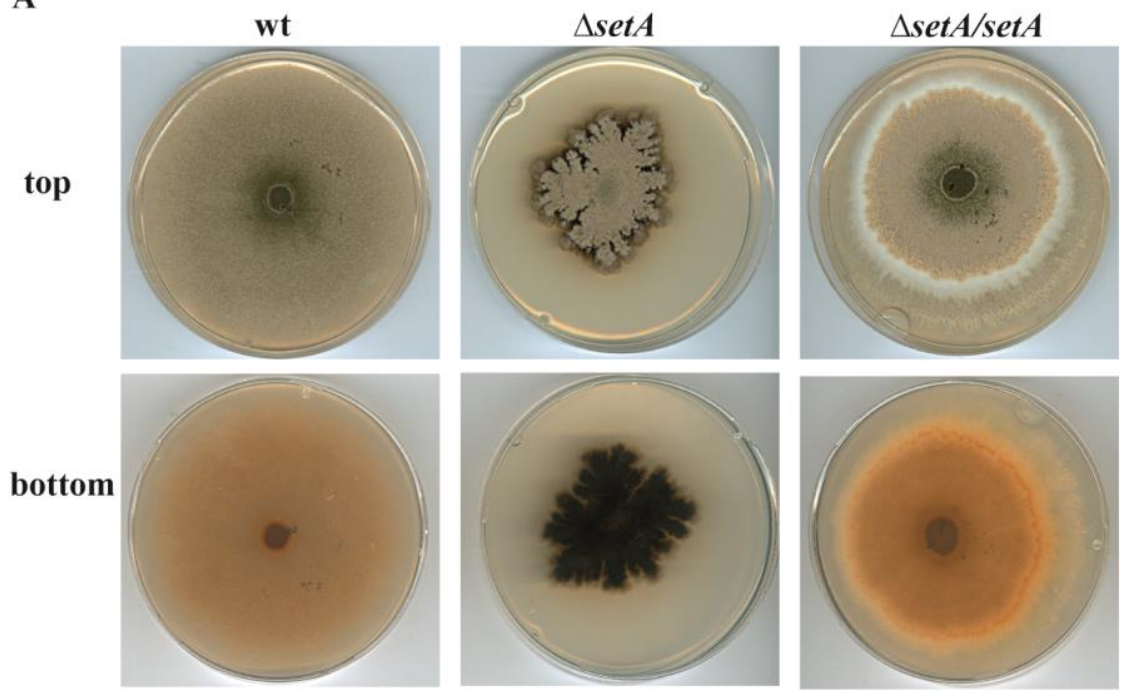

B
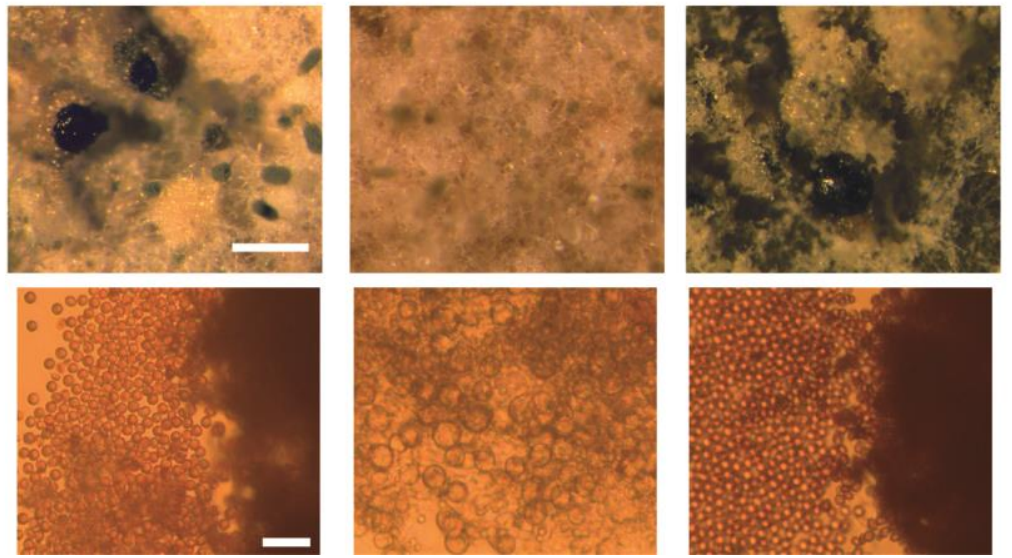

\section{Figure 23: Deletion of setA leads to an early block in sexual development.}

A Colony view of the wild type (wt), the $\operatorname{set} A$ deletion $(\triangle \operatorname{set} A)$ and the complementation strain $(\triangle \operatorname{set} A / \operatorname{set} A)$ from top (upper panel) and bottom (lower panel). 500 spores of each strain were pointinoculated on solid minimal medium containing appropriate supplements and grown for 7 days at $37^{\circ} \mathrm{C}$ under sexual development inducing conditions. B Upper panel: close up views of sexual fruit bodies. Scale bar $200 \mu \mathrm{m}$. Lower panel: microscopy view on squeezed fruit bodies or nests, respectively. Scale bar $20 \mu \mathrm{m}$ (modified published in (Harting et al., 2013)).

\subsubsection{SetA is required for proper conidiophore formation}

Next, the phenotype in asexual development was investigated. During illumination, where asexual development is favored, a significant growth reduction of the setA deletion strain compared to the wild type and the complementation strain was observed (Figure 24A and B). In addition, the colony morphology was disturbed, as the $\Delta$ setA strain showed a ragged, uneven growth phenotype when grown on minimal medium. 
A brown-red pigment produced by the setA deletion strain indicates defects in secondary metabolism (Figure 24A). A well-characterized metabolite of A. nidulans is the aflatoxin precursor sterigmatocystin. Therefore, the ability to produce this metabolite was investigated. It was found that under the tested conditions the $\Delta s e t A$ strain produced sterigmatocystin, although the amount of the metabolite produced appeared to be reduced (Figure 24C). Quantification of asexual spores revealed, that the conidia produced by the setA deletion strain are reduced to approximately $35 \%$ in comparison to the wild type strain. The defect was complemented by ectopical expression of SetA (Figure 24D).

Further experiments were performed to investigate whether the reduction in spore formation is a consequence of altered conidiophore morphology. Strains were grown on oblique, thin agar layers and observed under the microscope. This revealed an unusual defect in positioning and location of asexual structures. The strains were incubated for approximately $22 \mathrm{~h}$ at $37^{\circ} \mathrm{C}$. In the wild type and the complementation strain the asexual spores germinated and formed long, branched hyphae. 70 to $80 \%$ of the spores formed stalks from the developed hyphae. From these stalks a vesicle buds and on its top metulae, phialides and conidiospores develop (see chapter 1.4.3.). Only a small proportion of approximately $20 \%$ of wild type spores developed conidiophores directly on top of only short stalks. In contrast, this ratio is reversed in the mutant strain (Figure $25 \mathrm{~A}$ and $\mathrm{B}$ ). About $30 \%$ of the setA deletion mutant conidiophores originated from stalks. These stalks are aerial hyphae formed after branching. Approximately $70 \%$ of the setA deletion strain spores directly formed conidiophores, partially at the end of very long hyphae (Figure 25A and B). In general, branching of the hyphae appears to be disturbed in the $\Delta$ setA strain (Figure 25C). Some of the hyphae which ended in conidiophores could form new stalks with conidiophores (Figure 25D).

In order to investigate, whether the morphological and developmental abnormalities in the setA deletion strain might be caused by problems in nuclear distribution, the nuclear pattern within the hyphae was examined. A wild type strain, expressing a histone::rfp fusion was compared to a setA deletion strain carrying the same construct. Fluorescence microscopy revealed that the $\Delta s e t A$ strain shows a wild type like distribution of nuclei under vegetative growth conditions (data not shown). 
A

wt
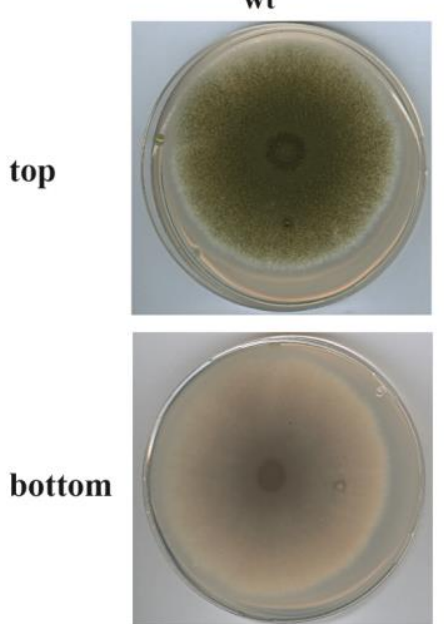

colony edge

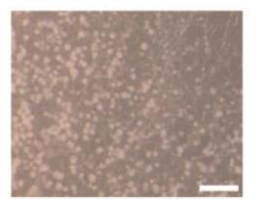

B

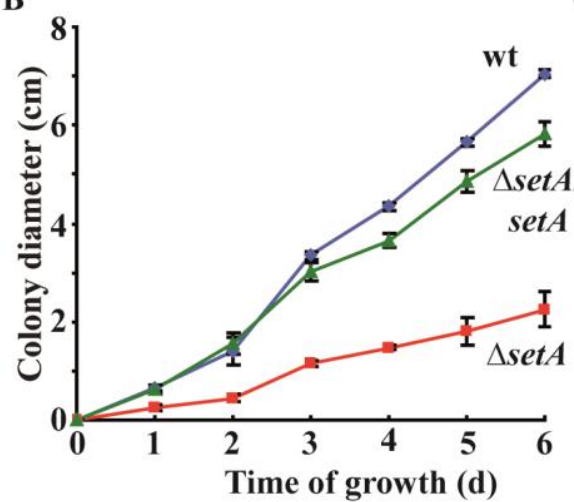

$\Delta$ setA
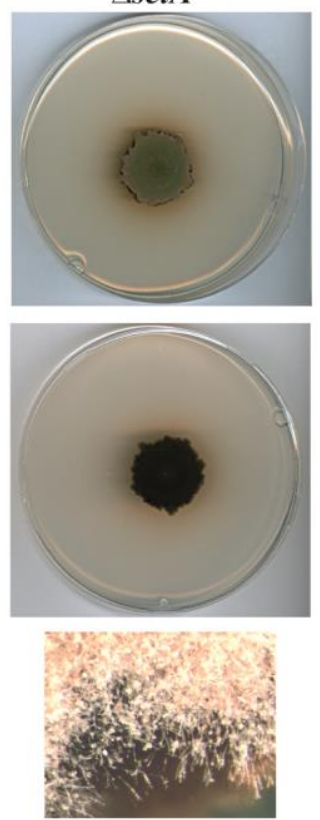

C

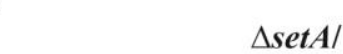

$\Delta \operatorname{set} A / \operatorname{set} A$
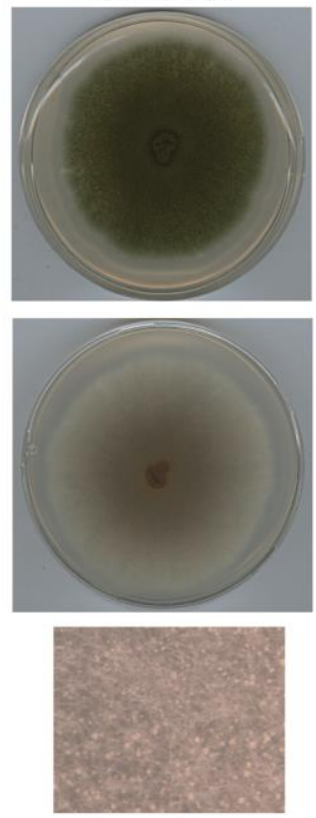

D
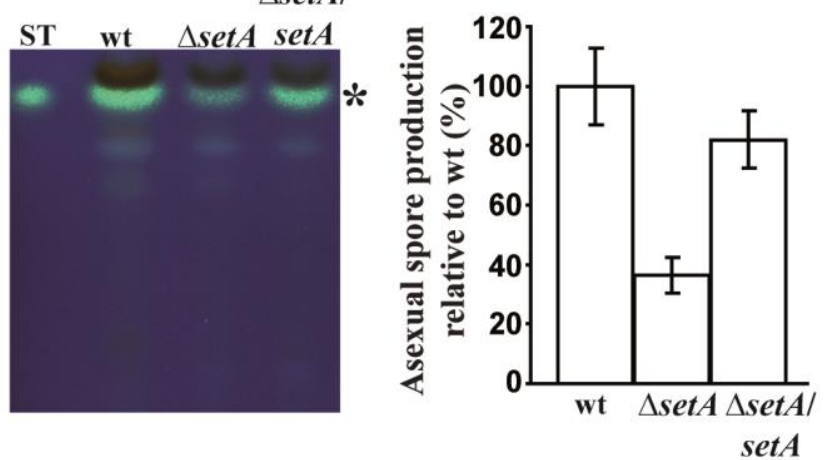

Figure 24: The setA deletion strain is impaired in asexual development.

A Asexual development of the setA deletion strain. 500 spores of each strain were point-inoculated on MM containing appropriate supplements and grown for 5 days at $37^{\circ} \mathrm{C}$ under asexual development inducing conditions. Colony view of the wild type (wt), the setA deletion $(\Delta s e t)$ and the complementation strain ( $\Delta$ set/setA) from top (upper panel) and bottom (middle panel). Lower panel: Close up views of the colony edge. Scale bar $200 \mu \mathrm{m}$. B Colony diameter of the wild type (wt), the setA deletion $(\Delta s e t)$ and the complementation strain $(\Delta$ set/setA $)$. Growth conditions as described for A. Colony diameter was measured daily. Data is derived from two independent experiments. C Sterigmatocystin production of the wild type $(\mathrm{wt})$, the setA deletion $(\Delta s e t)$ and the complementation strain $(\Delta$ set/setA $) .1 \times 10^{7}$ spores were spread on oat meal medium containing appropriate supplements and grown for 4 days at $37^{\circ} \mathrm{C}$ under asexual development inducing conditions. * marks the respective signal for sterigmatocystin. As standard purified sterigmatocystin (ST) was used. D Quantification of asexual spores formed by the wild type (wt), the setA deletion $(\Delta s e t)$ and the complementation strain $(\Delta$ set/set $A) .1 \times 10^{6}$ spores were plated on solid minimal medium containing appropriate supplements and grown for 3 days at $37^{\circ} \mathrm{C}$. Spore number was determined for five $0.5 \mathrm{~cm}^{2}$ sections per plate. Ratios were calculated relative to the wt spore number in \%. Data is derived from four experiments (modified published in (Harting et al., 2013)). 
These data show that the COMPASS complex is an important regulator of multicellular development and secondary metabolism in A. nidulans. The core subunit of the complex, SetA, functions in asexual development in the balance between the formation of conidiophores on foot cells or hyphal tips as starting point. The COMPASS complex might reflect an interface of the histone modification and the sumoylation pathway in the regulation of fungal development (published in (Harting et al., 2013)).

A

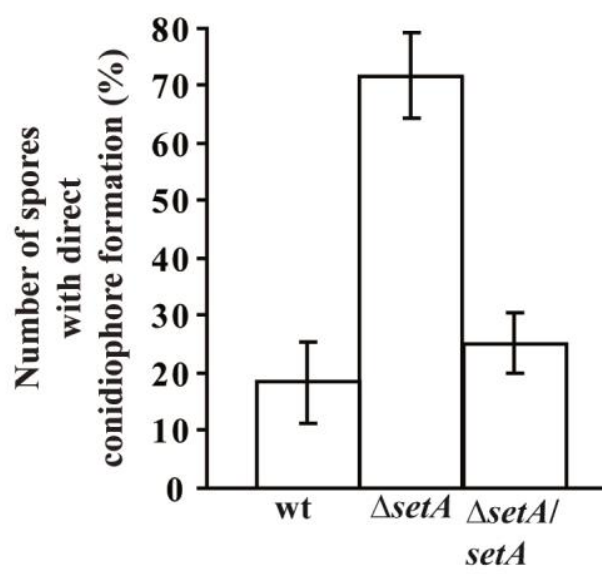

B

wt

$\Delta$ set $A$
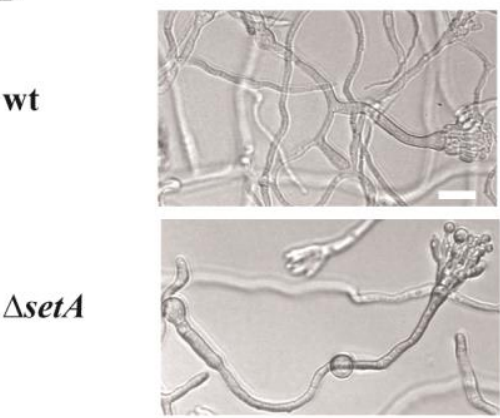

$\Delta$ setA/setA

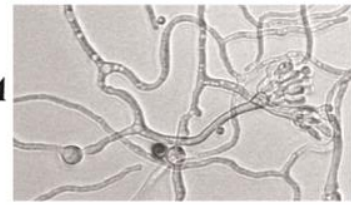

D

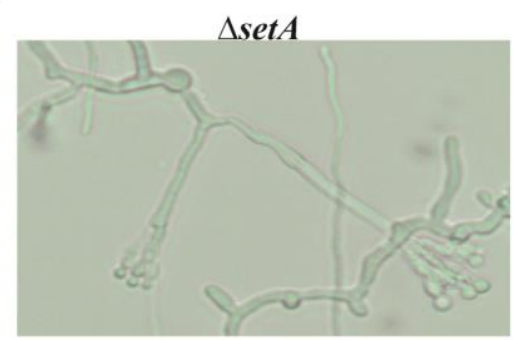

Figure 25: Deletion of setA leads to unusual positioning of the conidiophore.

A Quantification of conidiophores that directly grew from spores. Spores of the wild type (wt), the setA deletion $(\triangle \operatorname{set} A)$ and the complementation $(\triangle \operatorname{set} A / \operatorname{set} A)$ strain were directly inoculated on a thin agar layer on microscope slides. Conidiophores were pictured under microscope and the number of conidiophores that originate directly from spores was counted and ratios were calculated in \%. Data is derived from six experiments with 100 conidiophores each. B Representative conidiophores of the wild type (wt), the $\operatorname{set} A \operatorname{deletion}(\Delta \operatorname{set} A)$ and the complementation $(\Delta \operatorname{set} A / \operatorname{set} A)$ strain. Strains were grown as described in A. Scale bar $20 \mu \mathrm{m}$. C and D Pictures of single conidiophores of the setA deletion $(\Delta s e t A)$ strain to illustrate the potential branching defect $(\mathrm{C})$ as well as conidiophore formation at hyphae which ended in conidiophores (D) (modified published in (Harting et al., 2013)). 


\subsubsection{SetA overexpression allows normal development}

As deletion of setA has pleiotropic effects on fungal development, a setA overexpression strain was constructed to investigate the consequences of high levels of SetA in the cell. Therefore, the gene fused to $g f p$ was ectopically expressed in wild type background under the control of an inducible nitrate promoter. Ectopical integration of the plasmid was confirmed by Southern hybridization. Increased amounts of RNA were confirmed using qRT PCR with setA specific primers (data not shown). However, the GFP-SetA fusion protein could not be monitored in western hybridizations. No developmental defect in asexual and sexual development could be observed in both strains. Analyses of the codon usage within the protein revealed that it resembles the one of low transcribed proteins (Lloyd \& Sharp, 1991). This suggests a posttranscriptional regulation and therefore expression of setA under control of the nitrate promoter might lead to an elevated expression of RNA but not of protein, as some of the codons are rare within the cell. 


\section{Discussion}

\subsection{The neddylation network of Aspergillus nidulans}

Cycles of neddylation and deneddylation are regulating the activity of Ubiquitin E3 ligases and with this the ubiquitination of substrates (Merlet et al., 2009). The process of ubiquitination is important for various cellular processes, such as proteasomal degradation, transcription, endocytosis, DNA repair, membrane protein trafficking or signal transduction (Hochstrasser, 2009). In contrast to the sumoylation pathway, neddylation is essential for the viability of A. nidulans, as cells lacking either NeddH or its E2 conjugation enzyme UbcL are not able to survive (von Zeska Kress et al., 2012) (Figure 26). This is similar to other eukaryotic organisms as deficient neddylation is lethal at the pupal stage in D. melanogaster and leads to defects in embryogenesis in C.elegans and mice (Jones \& Candido, 2000, Mummery-Widmer et al., 2009, Tateishi et al., 2001). In contrast, a S. cerevisiae strain deficient for the Nedd8 homolog Rub1p, is viable (Liakopoulos et al., 1998).

Deletions of CRL subunits, such as CulA and SkpA are lethal for A. nidulans (von Zeska Kress et al., 2012) (Figure 26), demonstrating general importance of the ubiquitination reaction. In contrast, deneddylation is not important for fungal viability but is crucial for multicellular development in A.nidulans. Deletion of single subunits of the COP9 signalosome lead to a block in sexual reproduction and implications in metabolism and transcription (Braus et al., 2010), whereas asexual development is not severely affected. The formation of conidiospores is depending on the second fungal deneddylase, DenA. Additionally, there is an interaction of the two deneddylases which is also conserved in human (Christmann et al., 2013).

\subsubsection{DenA promotes cullin neddylation}

Similar to other ubiquitin-like proteins, the Nedd8 attachment pathway requires the action of Nedd8 E1, E2 and E3 enzymes (van der Veen \& Ploegh, 2012). The role of the two-component E3 ligase DcnA/RbxA was addressed in Aspergillus nidulans. In yeast, the RING protein Rbx 1p together with Dcn1p acts as E3 ligase for the neddylation of Cdc53p, the homolog of human Cullin1 and scaffold subunit of SCF complexes. The N-terminus of Rbx1p binds to Cdc53p, while the RING domain interacts with Nedd8-charged Ubc12p, the Nedd8 (Rub1p) E2 enzyme. Next, the 
RING domain is suggested to rotate to bring Ubc12p in connection with the lysine residue of Cdc53p that will be modified. Dcn1p can bind Cdc53p and reduce nonspecific Ubc12p-Rub1p discharge and assists in bringing the E2 enzyme into close proximity of the cullins lysine residue (Scott et al., 2010).

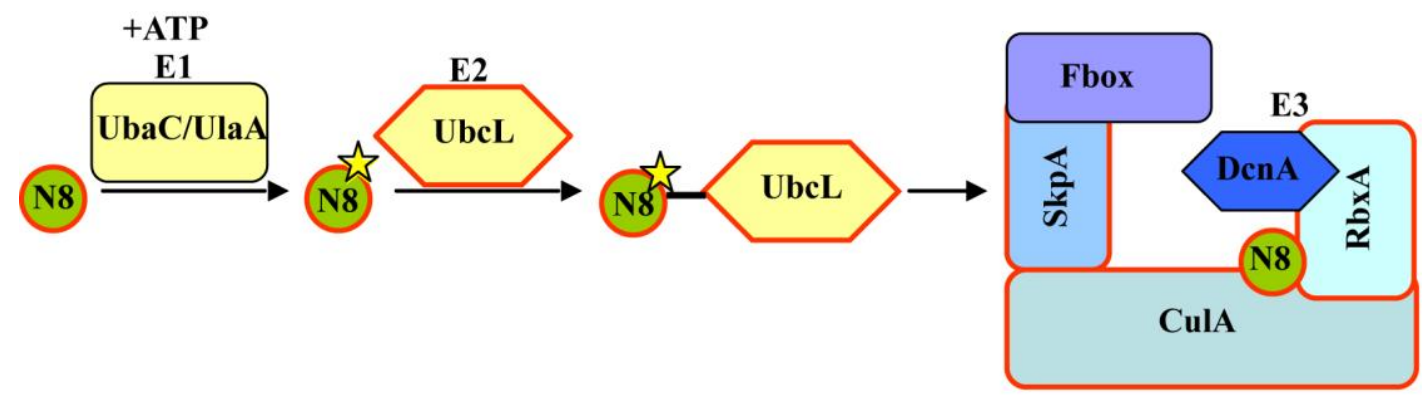

\section{Figure 26: The neddylation pathway in A. nidulans.}

The model shows the neddylation reaction in Aspergillus nidulans. NeddH (N8) becomes activated by the action of the UbaC/UlaA dimer in an ATP-dependent reaction. Next, it is conjugated to the E2 conjugation enzyme UbcL. In the last step, NeddH is bound to CulA of a SCF complex by the action of RbxA and DcnA. Red frames indicate proteins that were shown to be essential for fungal viability.

Interactions of A.nidulans DenA with the Ubc12p homolog UbcL, with NeddH and the RING protein RbxA were found, confirming a function in the neddylation pathway of the fungus. Western hybridizations with a $d \operatorname{cn} A$ deletion strain displayed a moderate reduction of cullin neddylation which was not affecting development under the investigated conditions. Therefore, it is suggested that A. nidulans can tolerate a slight reduction in cullin neddylation. One reason might be that ubiquitin ligase complexes containing either the Cullin2 or Cullin4 homolog, CulB or CulD respectively, can function to balance the reduction of CulA neddylation. Our western hybridizations did not distinguish between the three modified cullin proteins which have a similar size. Another possibility is that neddylation catalyzed by RbxA is sufficient to complete the developmental cycles under the tested conditions. An auxiliary function of DcnA E3 ligase activity, however, might be essential to survive stress and competitive conditions in nature. It is still elusive, whether DcnA specifically acts on CulA and if there are additional factors that support the NeddH E3 ligase activity of RING proteins in cullin neddylation. TAP-tagged NeddH was co-purified with its E1 activating enzyme $\mathrm{UbaC} / \mathrm{UlaA}, \mathrm{UbcL}$ as the E2 conjugation enzyme, RbxA, the three cullins CulA, 
CulB and CulD as well as SkpA and some F-box proteins (von Zeska Kress et al., 2012). DcnA was not identified in this screen, supporting the idea of an auxiliary function of DcnA, as the interaction demonstrated in vivo might be more transient.

The RING protein RbxA has two functions in cells: it catalyzes the neddylation reaction as E3 ligase and additionally functions in the ubiquitination process. The RING protein recruits ubiquitin-loaded E2 enzymes and therefore enables the binding of ubiquitin to a substrate protein, recognized by the Skp1-Fbox subcomplex of the CRL (Sarikas et al., 2011, Scott et al., 2010). Homokaryotic strains, carrying an $r b x A$ deletion in A. nidulans could not be obtained in this study, suggesting that the protein is essential for fungal viability as other parts of the SCF complex. It is speculative which role of RbxA is indispensable for the cell, the function as NeddH or Ubiquitin E3 ligase or both. The yeast homolog Rbx1p is essential (Seol et al., 1999), although the neddylation process itself is not, suggesting the function in ubiquitination to be important for cell viability in S. cerevisiae.

\subsubsection{The COP9 signalosome counteracts the accumulation of developmental SCF complexes}

The COP9 signalosome catalyzes the deneddylation reaction (Cope et al., 2002, Schwechheimer \& Deng, 2001). In plant cells, the CSN is involved in processes such as hormone signaling, regulation of growth and cell cycle as well as defense against pathogens (Stratmann \& Gusmaroli, 2012). In mammalian cells, the COP9 signalosome and the regulation of protein degradation is also connected to cancer (Lee et al., 2011b). An A. nidulans strain with a $\operatorname{csnE}$ deletion, accumulates several developmentally relevant Fbox proteins in a TAP-NeddH experiment (von Zeska Kress et al., 2012). Western hybridizations showed that the amount of neddylated cullins in a deneddylation deficient strain was increased. Overexpression of $f b x 15$ led to high and stable levels of the protein in wild type background but reduced and destabilized protein in a $\Delta \operatorname{csn} E$ strain. In human cell lines it was found that down regulation of Csn5 expression led to autoubiquitination of Fbox proteins resulting in an instability of the substrate specific subunits of the SCF complexes (Cope \& Deshaies, 2006). This suggests that a small subpopulation of Fbox 15 might be stabilized within a SCF complex and can be biochemically enriched, whereas the rest of the protein is more instable because of autoubiquitination. In the wild type background this process might be prevented by dynamic neddylation and 
deneddylation cycles. These cycles allow flexibility in the composition of SCF complexes as the substrate adaptor composed of SkpA and the Fbox proteins can be exchanged (Wei \& Deng, 2003). This recomposition of Ubiquitin E3 ligases might have an important impact on development, as it allows the degradation of specific target proteins, such as transcription factors, in a strictly regulated manner. The process of dis- and reassembly of SCF complexes might be supported by CandA. CandA binds to deneddylated cullin-RING subcomplexes, putatively stabilizing the basis and supporting the substrate adaptor exchange (Bornstein et al., 2006, Dubiel, 2009, Lo \& Hannink, 2006, Siergiejuk et al., 2009). However, it remains elusive, if A. nidulans CandA is connected to the regulation of Fbox 15 stability.

\subsubsection{The NeddH associated proteins AN4491 and AN4149 are dispensable for A. nidulans development}

The best characterized targets for the neddylation process are cullins but also other substrates were suggested. One example is the polyneddylation of histone 4 in response to DNA damage (Ma et al., 2013) (for details see chapter 4.2.2). However, it is proposed that neddylated substrates other than cullins are only weakly expressed and/or neddylated transiently and therefore difficult to identify (Rabut \& Peter, 2008). In experiments with TAP-tagged NeddH two so far uncharacterized Aspergillus proteins, AN4491 and AN4149, were identified (von Zeska Kress et al., 2012). AN4491 was co-purified in wild type and $\operatorname{csn} E$ deletion strain, whereas AN4149 was only found in the deneddylase deficient strain. There are several possibilities for an interaction of the two proteins with the neddylation or ubiquitination machinery. For example, they could be neddylated proteins. Additionally, the proteins could be substrates for the degradation machinery and might contribute to the phenotypes of developmental Fbox proteins which were found in the same experiments. AN4491 and AN4149 could also interact with any of the biochemically enriched proteins. A yeast two hybrid approach to identify interactions with the neddylation machinery did not give any results (data not shown). Therefore, putative interactions of the two proteins with the processes of neddylation/ubiquitination remain elusive. The influence of AN4491 and AN4149 on A. nidulans development was investigated in this study but both corresponding deletion strains displayed normal development under laboratory conditions. Therefore, the distinct functions of the proteins and their connection to the neddylation/ubiquitination machinery remain elusive. 


\subsection{The SumO network of Aspergillus nidulans}

Posttranslational modification of target proteins by sumoylation controls the localization, activity and interaction of eukaryotic proteins. In A.nidulans, sumoylation has a key role in multicellular differentiation (Laubinger, 2008, Szewczyk et al., 2008, Wong et al., 2008). It is required to repress sexual development during illumination and to accelerate asexual spore formation. Additionally, functional sumoylation is also important for the completion of sexual fruit body formation and coordinated secondary metabolism. It is still elusive which developmental factors are directly or indirectly regulated through sumoylation. In this study, members of this dynamic fungal SumO network (Figure 27) were identified by the enrichment of sumoylated proteins, followed by mass spectrometry identification. In addition, genetical approaches were used to characterize the sumoylation/desumoylation machinery and putative targets.

The TAP method used for the identification of putative interaction partners and substrates of sumoylation is a two-step purification with which specific interaction partners can be identified. Proteins that unspecifically bind to the reagents used for enrichment or those that are present in high amounts in the cell can be identified as false positives. These should be excluded by control experiments with the corresponding parental strains. However, sumoylated or sumo-interacting proteins might have been therefore excluded from the list due to their expression levels or binding characteristics. One example is the SumO E1 activating enzyme UbaB. In addition, identified proteins might also be interaction partners of sumoylated proteins and are not modified themselves. It can not be excluded that some proteins were interacting with the tag instead of SumO itself. This requires further investigation of the putative role of the co-purified proteins within the sumoylation network. In the sumO-isopeptidase deficient strain, non-tagged SumO was identified in the control strain, suggesting, that some proteins found in the control are also sumoylated but considered as unspecific possibly due to high expression levels. Therefore, these SumO substrates are not included in the data set.

The TAP::SumO protein was partially functional. An impairment of the sumoylation function might be due to the fact that the tag could be disturbing for individual SumO interactions. However, western hybridization and partial complementation of the sumO deletion phenotype suggests that the attachment is possible. The construct used for TAP experiments interferes with the neighboring 
gene of sumO, AN1192. A deletion strain of the AN1192 encoding gene was constructed to investigate possible consequences. The strain lacking AN1192 was behaving like the wild type strain, suggesting that the deletion does not have a global impact on development and/or protein expression.

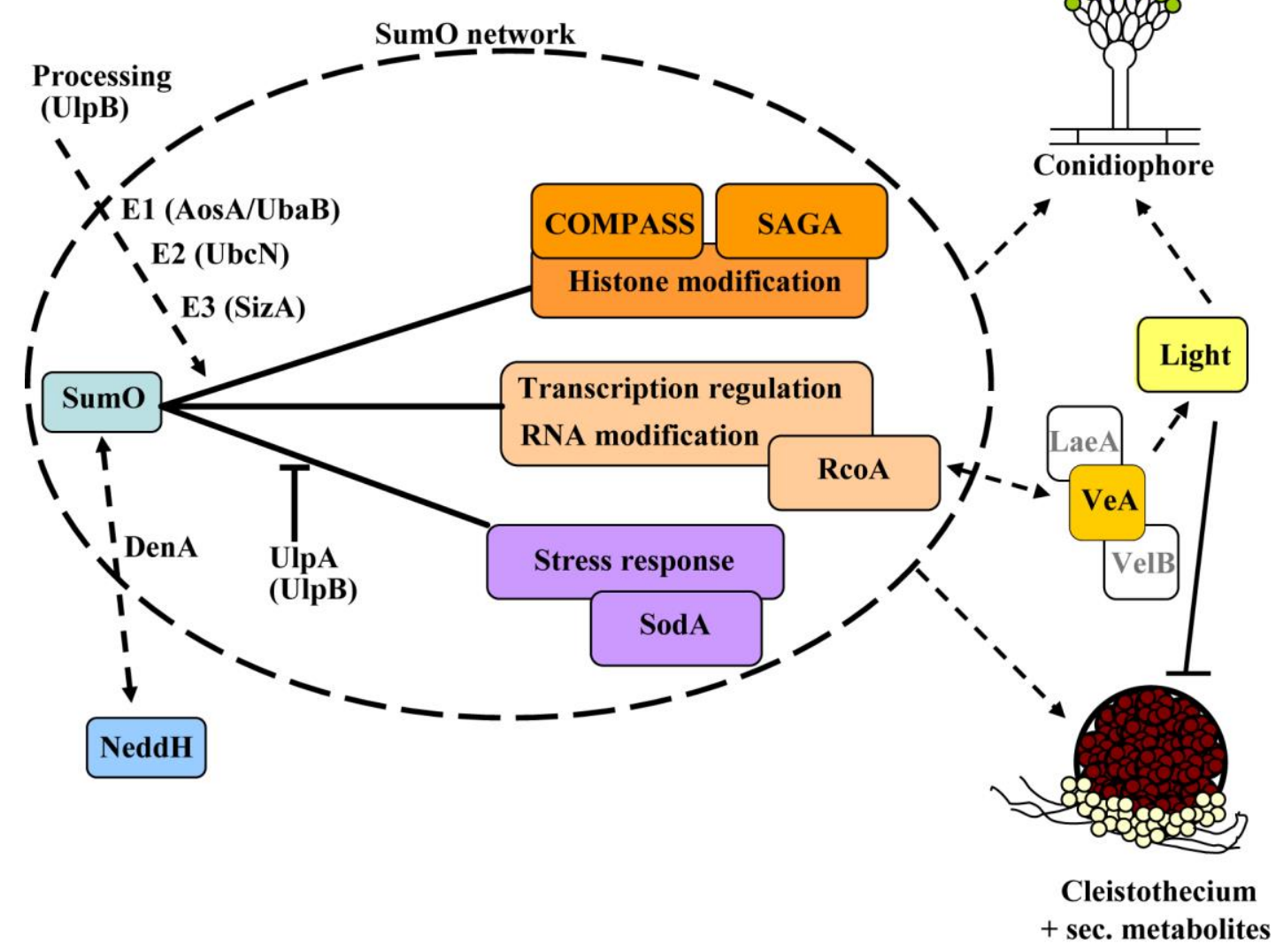

Figure 27: The SumO network of Aspergillus nidulans.

The fungal SumO network including sumoylation and desumoylation of putative substrates are combined in a model with the substrates which were identified in this study. Downstream effects depend on environmental stimuli and result in A. nidulans in the formation of sexual fruit bodies, the cleistothecia, or asexual structures, the conidiophores. Sumoylation is required for sexual reproduction, enhanced conidiophore formation and accurate response to light. The SumO precursor which is processed ( $\mathrm{UlpB}$ ), is activated by the heterodimer $\mathrm{Aos} \mathrm{A} / \mathrm{UbaB}(\mathrm{E} 1)$ and transferred to the conjugation enzyme UbcN (E2). With the help of E3 ligases (such as SizA), SumO becomes attached to target substrates. Potential target proteins for sumoylation in A.nidulans are histone modifiers as for example the COMPASS complex and the SAGA complex, transcriptional regulators, such as RcoA, RNA maturation proteins, and stress response enzymes, such as SodA. The SumO network possibly influences light dependent regulation of development via interplay of RcoA with VeA which is part of the trimeric velvet complex VeA-LaeA-VelB. 
Homologs of many proteins that were enriched have been connected to the sumoylation process in other organisms, as for example the enzymes of the sumoylation pathway or the topoisomerase I (Wohlschlegel et al., 2004). This suggests specificity of the experiments and allows to link the sumoylation network to fungal development, gene expression, stress response, ubiquitination and neddylation in A. nidulans.

\subsubsection{The SumO network and multicellular development}

Multicellular fungal development requires the entire sumoylation pathway including SumO, the SumO processing enzyme UlpB, the E1 SumO activating enzyme AosA/UbaB as well as the E2 conjugating enzyme UbcN. The phenotype of the corresponding deletion strains share similar pleiotropic effects on fungal cell differentiation. This is different for the deletion strain of the gene encoding the SumO E3 ligase SizA, as it only affected the fungal light response, but no other developmental program. This might be due to redundant and/or additional functions of other SumO E3 ligases in the fungus. A second protein with a Siz/PIAS RING finger-like domain is present in A. nidulans, the yet uncharacterized protein AN4497. Sumo E3 ligases of the Siz/PIAS family often share a common domain organization, composed of SAP, PINIT and SP-RING domain (Figure 28). In addition the proteins display Sumo-interaction motifs and a variable S/T-rich domain (Rytinki et al., 2009). SizA has the same domain structure as the Siz/PIAS protein but misses an LxxLL motif within the SAP domain. This motif is important for the interactions with nuclear receptors. Additionally, it is significantly smaller compared to the yeast protein Siz1p. Yeast Siz1p deletion strains are viable and defective in septin sumoylation (Takahashi et al., 2001b).

The second important E3 ligase in yeast, Siz2p, is involved in the regulation of teleomere positioning (Ferreira et al., 2011) and does not have a homolog in A. nidulans. Neither does the E3 ligase Cst9p from budding yeast which is required for synaptonemal complex formation (Agarwal \& Roeder, 2000). In contrast, a putative homolog of the yeast Sumo (Smt3p) E3 ligase Mms21p is expressed in the fungus which is AN10240. Deletion of the Mms21p encoding gene in S. cerevisiae led to an increased sensitivity against DNA damage stress and defects in nucleolar integrity and telomere functions (Zhao \& Blobel, 2005). Both Aspergillus proteins, AN4497 and AN10240, might function as additional E3 ligases in the SumO 
pathway. The putative homolog of Mms21p, AN10240, was co-purified with TAP::SumO in the ulpA deletion strain. But as only proteins, at least identified in two independent experiments were considered as verified, it is not displayed in table 6. However, the enrichment of AN10240 in one experiment supports the assumption that it might function as SumO E3 ligase. Probably it is needed for the sumoylation of a smaller range of substrates compared to SizA which would explain the reduced occurrence of the protein in the experiments.
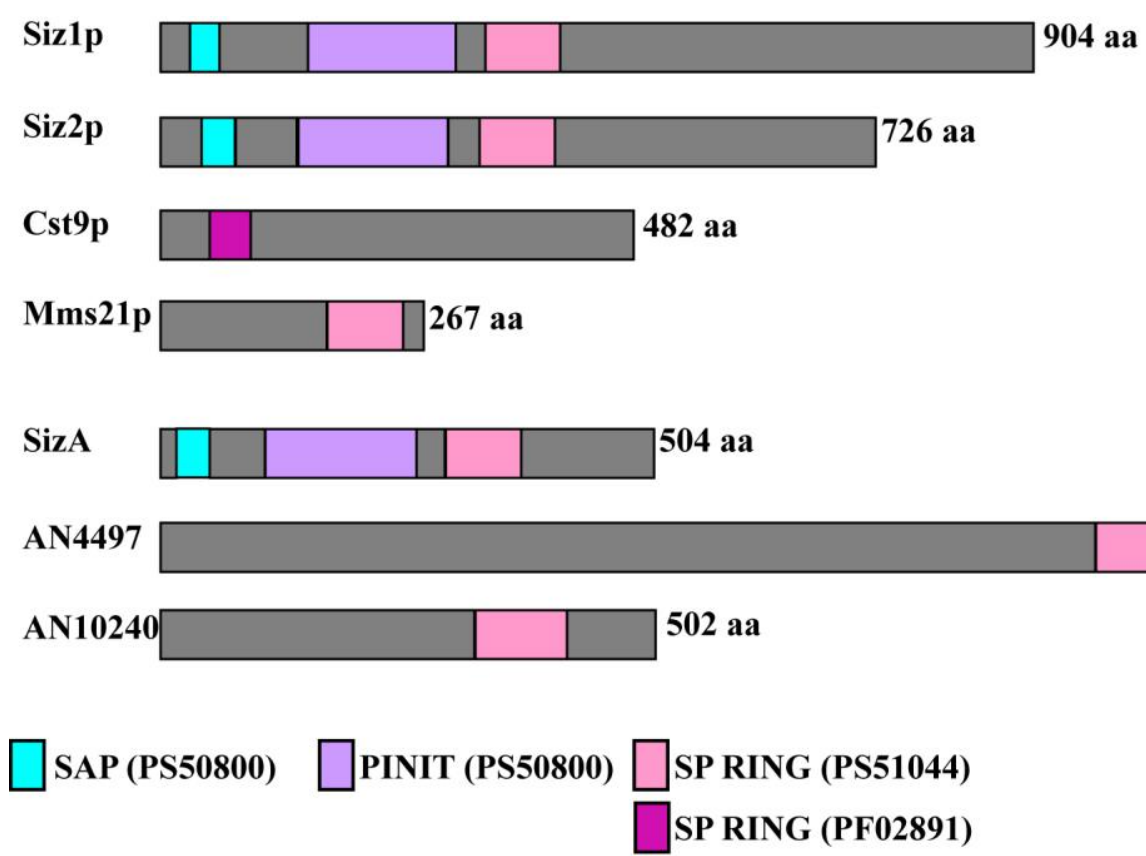

Figure 28: Sumo E3 ligases of S. cerevisiae and A. nidulans.

Domain structure of yeast Sumo (Smt3p) E3 ligases Siz1p, Siz2p, Cst9p and Mms21p in comparison with the A. nidulans SumO E3 ligase SizA and the two additional putative E3 ligases AN4497 and AN10240. In brackets the InterProScan signatures are shown.

Fungal development requires both aspects of the SumO network, sumoylation and desumoylation of substrates. In this study, two SumO isopeptidases were identified as part of the SumO network. UlpB is the processing enzyme but might be also involved in the cleavage of SumO from substrates. In yeast this function is suggested for Ulp1p, whereas Ulp2p is a desumoylating enzyme (Li \& Hochstrasser, 1999, Li \& Hochstrasser, 2000). Wss1p was additionally described in yeast. It cleaves Sumo-ubiquitin as well as Sumo chains in vitro and interacts with Sumotargeted ubiquitin ligases (Mullen et al., 2010). AN0304 represents a putative A. nidulans homolog which was not investigated so far. UlpA is a desumoylating 
enzyme of A. nidulans. The impact of UlpA on the regulation of development is less severe in comparison to sumoylation enzymes such as the E1 activating or the E2 conjugating enzyme but still significant. This might be due to additional desumoylating functions of UlpB, the Wss1p homolog or other so far not identified SumO isopeptidases. The homolog of yeast Wss1p might also be a connection between the pathways of sumoylation and ubiquitination in A. nidulans (see also chapter 4.2.2.). However, the change between sumoylated and non-sumoylated states of substrates is of significant importance for fungal development.

\subsubsection{The SumO network and the velvet complex}

In A. nidulans, LaeA is a central regulator of secondary metabolism that is also important for light-dependent regulation of development (Bayram \& Braus, 2012, Bayram et al., 2008b, Bok \& Keller, 2004). The protein binds to a VelB/VeA dimer to form the trimeric velvet complex (Bayram et al., 2008b). VeA shows lightdependent expression and nuclear localization and connects the light sensing to the developmental program (Bayram \& Braus, 2012). The Sumo network of A. nidulans might be linked to the velvet complex by RcoA. RcoA was co-purified with TAP::SumO and could be a downstream target of velvet signaling. The $r c o A$ deletion strain is blocked in sexual development dependent on VeA (Todd et al., 2006). The yeast homolog of RcoA, Tup1p, was found to act in the repression of genes together with Cyc8p (Smith \& Johnson, 2000). The homolog of Cyc8p, SsnF, was also identified in our screen. Sumoylation of one or both of the two proteins might be involved in the regulation of developmental genes (see chapter 4.2.3.2.) in response to light.

In $N$. crassa it was shown that the induction of development depending on light signals required a functional SAGA complex (Grimaldi et al., 2006). In our screen, several putative subunits of the Aspergillus SAGA complex were identified which might be an additional interface for the SumO network, photoinduction of development and gene expression (see chapter 5.2.3.).

\subsubsection{The SumO network and stress response}

Fungal development is also influenced by other environmental conditions besides light. A. nidulans has to adapt to various stress conditions to survive in a complex habitat as the soil. Development is therefore also connected to stress 
response pathways in the cell, as shown for the NADPH oxidase NoxA. Deletion of the corresponding gene results in a block of fungal sexual reproduction (Lara-Ortiz et $a l ., 2003)$. The balance of reactive oxygen species within fungal cells represents an important internal trigger of development and hyphal growth (Lara-Ortiz et al., 2003, Semighini \& Harris, 2008). This might be another link to the sumoylation network, as the superoxide dismutase SodA (Oberegger et al., 2000) and the homolog of yeast $\mathrm{Ntg} 1 \mathrm{p}$ were identified in our screen. $\mathrm{Ntg} 1 \mathrm{p}$ is important for the repair of oxidatively damaged DNA (Bruner et al., 1998). SodA is specifically upregulated in response to iron starvation (Oberegger et al., 2000). The yeast homolog Sod1p was shown to be required for the detoxification of oxygen radicals (Bermingham-McDonogh et al., 1988) and it was suggested to be modified with the yeast Sumo protein (Smt3p) (Hannich et al., 2005). Deletion of the gene encoding Sod1p leads to an increased sensitivity against oxidative stress and the mutant displayed mitochondrial defects. Sod1p is therefore connected to cell aging of yeast (Longo et al., 1996, O'Brien et al., 2004). Mutations in human Sod1 were brought in context with the neurodegenerative disease ALS (âmyotrophic lateral sclerosis) (Pickles \& Vande Velde, 2012), underlining the importance of the proper regulation of oxidative stress response in cells. Modification of Sod1 with Sumo1 in human was shown to lead to an increased protein stability and aggregation (Fei et al., 2006), suggesting a regulatory impact of Sumo modification on oxidative stress response. The role of SodA sumoylation in A. nidulans might therefore be an interesting connection between the SumO network and developmental regulation in response to stress.

\subsubsection{The SumO network and other UBL pathways}

Proteins can be modified by Sumo and ubiquitin at the same lysine residue resulting in a competitive situation of the two pathways. Different modifications of the same protein can result in different consequences for the substrate. Stability, localization or activity of a target protein can be affected. Interactions of two UBL pathways also include the modification of enzymes of the pathway of the other modifier. Additionally, some enzymes can function in different pathways (Praefcke et al., 2012). One example is the human protein Topors which can function as Ubiquitin and Sumo E3 ligase (Rajendra et al., 2004, Weger et al., 2005).

Sumo-targeted ubiquitin ligases (STUbLs) are conserved in eukaryotes. STUbLs recognize sumoylated proteins by SIM motifs and interact with ubiquitin E2 
conjugation enzymes through RING domains. This leads to the attachment of Ubiquitin onto the Sumo protein (Praefcke et al., 2012). The ubiquitination can lead to proteasomal degradation of the Sumo modified protein (Uzunova et al., 2007). No homolog could be identified in A. nidulans for the yeast STUbL Hex3p (Slx 5p). This is different for the second STUbL, Ris1p (Uls1p) that shares similarity with the uncharacterized protein AN5483. Several subunits of the proteasome were identified through mass spectrometry after enrichment with TAP::SumO in this study which hints to a connection between sumoylated proteins and the $26 \mathrm{~S}$ proteasome. One possibility is that ubiquitin serves as degradation signal for some sumoylated substrates. Also the homolog of the yeast ubiquitin E1 activating enzyme Uba1p was biochemically enriched with SumO. It is also possible that the protein is sumoylated itself, as it has four putative sumoylation motifs. The homolog of a STUbL could not be identified in our screen which might be due to transient interactions or low expression levels of the protein. Additionally, nothing is known so far about STUbLs in A. nidulans. In general, it is thought that Sumo chains are recognized by the ligases (Praefcke et al., 2012). As in Aspergillus no lysine residue within a consensus motif is present and chain formation was not investigated, it will be a task for future research to further address the crosstalk between the ubiquitination and sumoylation network.

Another close relative to ubiquitin is the protein Nedd8. An interplay of sumoylation/desumoylation with the Nedd8 network is possible. High expression levels of the deneddylase DenA can partially suppress developmental defects caused by the lack of the SumO isopeptidase UlpA. In contrast, the desumoylation defects in the ulpA deletion strain were not complemented. The molecular interplay is yet elusive but might include the second A.nidulans deneddylase, the COP9 signalosome. The CSN is involved in secondary metabolism, hormone level regulation, oxidative stress response and light control (Braus et al., 2010) and physically interacts with DenA (Christmann et al., 2013). The SumO network might be linked to the COP9 signalosome through the DenA-COP9 interaction which seems to be conserved in eukaryotes (Christmann et al., 2013).

All three posttranslational modifications, sumoylation, neddylation and ubiquitination are involved in histone modification and stress response, leading to significant changes in the surface of the involved proteins with a potential impact on the respective functions (Du, 2012, Jackson \& Durocher, 2013, Ma et al., 2013, 
Trujillo et al., 2011) (Figure 29). Sumo, Nedd8 and Ubiquitin show a specific enrichment at sites of DNA damage (Ma et al., 2013, Morris, 2010). Histone 4 becomes polyneddylated upon DNA damage stress via the Ubiquitin E3 ligase Rnf111 and the Nedd8 E2 enzyme Ube2M. This modification can be recognized by Rnf168 which functions as E3 ligase in histone ubiquitination. Histone polyubiquitination recruits important factors as the breast cancer predisposition protein Brcal or 53BP1 (Ma et al., 2013). Brcal is another ubiquitin E3 ligase that functions in several DNA repair complexes, although its direct function is elusive. The ubiquitination function of Brcal is connected to its sumoylation, making it a Sumo-regulated ubiquitin ligase (SRUbL) (Morris et al., 2009). Additionally, the accumulation of Rnf168 is dependent on the function of the Sumo E3 ligase Pias4 (Morris, 2010) (Figure 29).

A similar regulatory interplay of the three modifiers might link histone modification and response to DNA damage in A. nidulans. The fungus lives in the soil but exposes aerial hyphae and the air-borne conidia to light. DNA damage response in the fungus is connected to light-dependent regulation of development via the photolyase-like protein CryA. CryA is a dual functional protein. As a blue light receptor it is negatively regulating sexual development and additionally functions in DNA repair after UV-damage (Bayram et al., 2008a). The role of posttranslational modifications as sumoylation, ubiquitination or neddylation in A. nidulans DNA damage response is an attractive research field where preliminary data suggested a connection. Deletion of COP9 signalosome deneddylase subunits resulted in an increased sensitivity against DNA damage inducing agents. mRNA amounts for $\operatorname{csn} E$ and $\operatorname{csn} D$, encoding two COP9 signalosome subunits, were increased under DNA damage stress inducing conditions. A genetical interaction of this deneddylating complex with enzymes involved in DNA repair has been proposed (Lima et al., 2005). SumO is supported as an additional player, because the deletion of Aspergillus sumO resulted in strains displaying an increased sensitivity against DNA damage inducing agents (Laubinger, 2008, Wong et al., 2008). This supports a critical role for neddylation/deneddylation as well as sumoylation/desumoylation for an accurate cellular stress response pathway. It is currently unclear how much of this combined modification activity is involved in controlling the function, the half life or the localization of target proteins. 


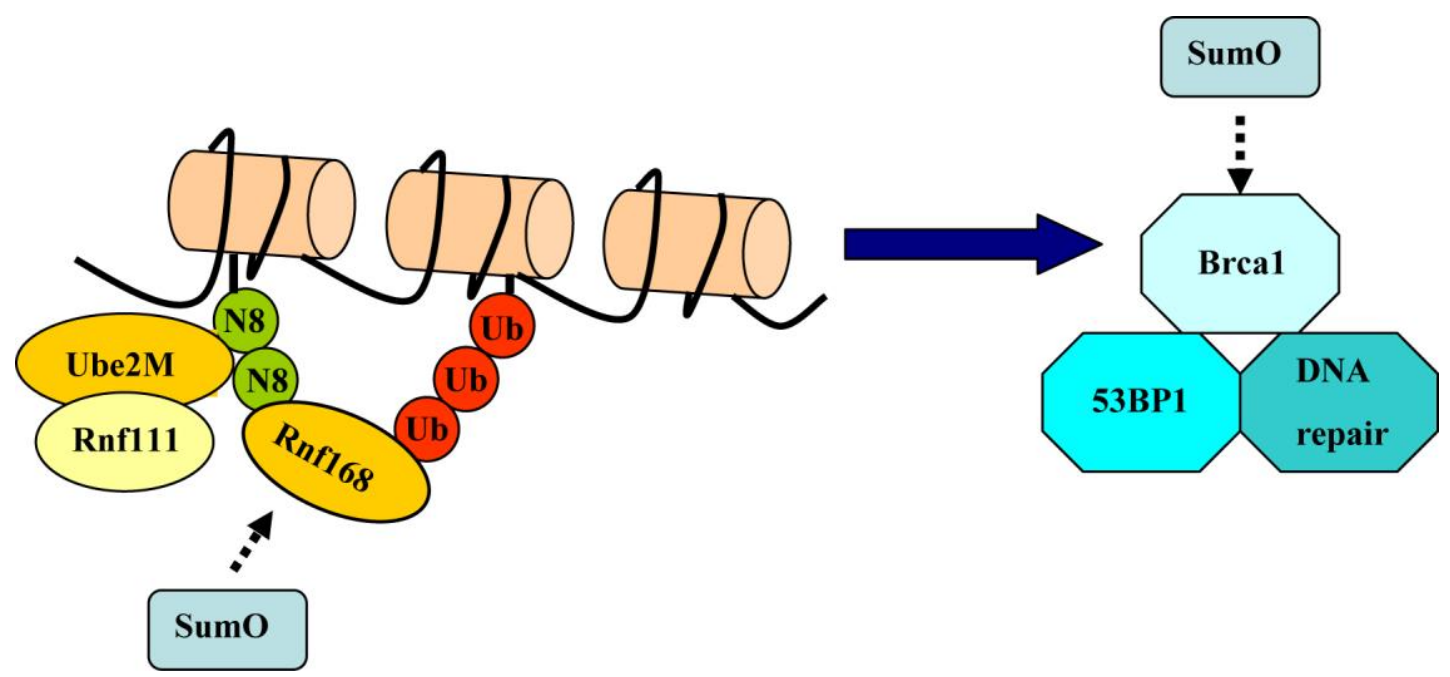

Figure 29: Interplay of neddylation, ubiquitination and sumoylation in DNA repair.

The model displays the crosstalk of the neddylation, ubiquitination and sumoylation networks in DNA damage response. Histones become polyneddylated by the action of the Nedd8 E2 enzyme Ube2M and the Ubiquitin E3 ligase Rnf111. The neddylation mark can be recognized by the E3 Ubiquitin ligase Rnf168 that functions in histone ubiquitination. These signals lead to the recruitment of proteins involved in DNA damage repair, such as Brca1 and 53BP1. The functions of Rnf168 and Brca1 are also influenced by sumoylation. Modified from (Ma et al., 2013).

\subsubsection{The SumO network and gene expression}

The transcriptional machinery in eukaryotes depends on the modification of histones, including phosphorylation, methylation, acetylation, ubiquitination and sumoylation. As consequence of the attachment of these groups or proteins, gene expression can be activated or repressed. In A. nidulans, histone modifications have been proposed to link development and secondary metabolism. LaeA, a subunit of the heterotrimeric velvet complex, is a putative histone methyltransferase that is also involved in development (Bayram \& Braus, 2012). Another example is the single fungal $\underline{S}$-adenosylmethionine synthetase (SasA) encoded in the A. nidulans genome. sasA is essential for fungal viability. Overexpression of SasA led to a block in sexual development and an impaired secondary metabolism (Gerke et al., 2012).

Sumoylation is often connected to transcriptional repression (Gill, 2005). Lowlevel sumoylation of transcriptional regulators can support the binding of additional factors, such as histone deacetylases (Gill, 2005). This could result in an inhibitory complex or in remodeled chromatin which efficiently represses transcription (Geiss- 
Friedlander \& Melchior, 2007). Additionally, there are examples for a transcription activating function of sumoylation (Lyst \& Stancheva, 2007). The A. nidulans Sumo network includes several possible transcriptional regulators which might contribute to the pleiotropic phenotypes of the sumO deletion strain.

\subsubsection{The SumO network and transcription factors}

The A. nidulans SumO network includes several possible transcription factors. Despite putative subunits of transcription factor complexes (AN2181, AN3907, AN5498), other transcriptional regulators were co-purified with SumO, such as SrrA (stress response regulator A) that acts as a response regulator. This protein is presumably involved in control of colony shape, conidiophore production and oxidative stress response (Hagiwara et al., 2007, Vargas-Perez et al., 2007). Other putative transcription factors which were identified in our screen have not been characterized so far in A. nidulans and do not have well-conserved and/or characterized S. cerevisiae homologs. One of these is AN0671 that contains a leucine-zipper domain. Another one is the protein AN12489 that has a zinc finger motif as well as a domain that is typical for fungal transcription factors. Additionally, a protein that is similar to the $S$. pombe protein Sre2p, AN7170, was identified. Sre2p is a predicted membrane-tethered transcription factor. The Aspergillus homolog was not characterized so far but it has a helix-loop-helix motif which is present in a number of transcription factors. The transcript of the gene is upregulated in response to DNA damage stress (Malavazi et al., 2006). As many of the identified putative transcription factors have not or not fully been characterized in A. nidulans their function in development and the connection to the SumO network is still elusive.

\subsubsection{The SumO network and gene repression by RcoA-SsnF}

The yeast proteins Tup1p and Cyc8p are conserved, mobile repression elements which interact with DNA binding proteins and repress $\sim 3 \%$ (in total more than 150) of the S. cerevisiae genes. Both proteins are not essential for yeast viability but show different phenotypes. The repressive function affects cellular processes such as DNA damage response, sporulation, meiosis, carbon metabolism, and flocculation. For repression of genes, the Tup1p-Cyc8p complex interacts with various DNA-binding proteins (Smith \& Johnson, 2000). The repressive function can be achieved by the recruitment or interaction with other factors, such as histone 
deacetylases, as for example the Sin3p-Rpd3p histone deacetylase complex (Watson et al., 2000). The homologous proteins in A.nidulans, RcoA, SsnF and the Sin3p homolog AN1546 were co-purified with SumO. In addition, the three yeast proteins were suggested to be modified or interact with $\mathrm{Smt} 3 \mathrm{p}$, the yeast Sumo protein (Denison et al., 2005, Hannich et al., 2005, Panse et al., 2004, Wohlschlegel et al., 2004, Zhou et al., 2004). In A. nidulans, ssnF is essential and RcoA is involved in important developmental processes as asexual and sexual development. This suggests that the functions of the two proteins are not completely overlapping (Garcia et al., 2008, Hicks et al., 2001, Todd et al., 2006). A regulatory interplay of the SumO network with the RcoA-SsnF corepressor system could influence the expression of various genes in A.nidulans. An altered gene repression could contribute to the pleiotropic phenotypes of the sumO deletion strain.

\subsubsection{The SumO network and the SAGA complex}

The SAGA complex is a highly conserved transcriptional co-activator that influences the expression of a number of genes in S. cerevisiae. It consists of four basic subcomplexes: the histone acetyltransferase (HAT) module with the catalytic subunit Gcn5p, the deubiquitinating (DUB) module with the enzyme Ubp8p, the SPT and the TAF module (Figure 30) (Samara \& Wolberger, 2011). Both, the deubiquitination as well as the HAT activity, contribute to the remodeling of the chromatin structure. Additionally, the SAGA complex can regulate gene expression by interaction with the TATA- inding protein (TBP) (Bhaumik, 2011). Several putative Aspergillus subunits of the SAGA complex were identified as possible members of the SumO network in this study. This includes the complete HAT module: GcnE (AN3621), a protein similar to Sgf29p (AN0668), a protein similar to Ngg1p/Ada3p (AN0440) and AdaB (AN10763). In addition two subunits of the SPT module were identified, the putative homologs of Spt7p (AN4894) and Spt20p (AN0975/RfeE). Also the putative homolog of Taf12p (AN2769) was found (Figure 30A).

Gcn5p, the core component of the HAT module of the SAGA complex, was shown to be modified by Smt3p. Sumoylation of neither Gcn5p alone nor the trimer Gcn5p-Ada2p-Ngg1p/Ada3p did affect the enzymatic function in vitro and mutation of the major sumoylated lysine at position 25 did not have effects in vivo. In contrast, linking of Smt3p to the N-terminus of Gen5p, mimicking constant sumoylation, led 
to growth defects on 3-aminotriazole containing medium similar to a gcn5 deletion strain accompanied by a reduced transcription of the SAGA complex activated gene TRP3. As a result of these experiments, it was suggested that the sumoylation of Gcn5p leads to gene repression (Sterner et al., 2006). The lysine residue that is sumoylated in Gen5p is not conserved in A. nidulans GcnE. Instead the protein contains a putative sumoylation motif surrounding lysine 37 , located at the $\mathrm{N}$ terminus of the protein similar to the sumoylation motif in Gcn5p. Several putative homologs of Gcn5p in different Aspergillus species are highly conserved, including the sumoylation motif of GcnE (Figure 30B). This supports the idea that the SAGA subunit could be also sumoylated in Aspergillus. However, it is not clear whether the other subunits that were co-purified with TAP::SumO are also sumoylated or were only bound to the putatively sumoylated GcnE.

It was shown that the putative SAGA complex subunits GenE and AdaB in A. nidulans are required for growth and asexual development. The conidiophore structure in corresponding deletion strains was disturbed. Both strains developed stalks and vesicles but without metulae. Additionally, stalks and vesicles with few phialides were formed directly from vegetative mycelium. This phenotype was accompanied by low levels of histone 3 acetylation but no effect on chromatin remodeling was observed (Reyes-Dominguez et al., 2008). Interestingly, it was shown that the deletion of the $N$. crassa homolog of $g c n 5, n g f-1$, led to a deficiency in histone 3 lysine 14 (H3K14) acetylation and a loss of photoinduction of development. The acetylation of H3K14 was found to be dependent on the blue light receptor White Collar-1 (WC-1) (Grimaldi et al., 2006). This directly links the acetylation process catalyzed by the SAGA complex to light-dependent development, an important process in A. nidulans, which is disturbed in the sumO deletion strain. In the fungus, blue light receptors are LreA and LreB (Purschwitz et al., 2008) and the photolyase-like protein CryA (Bayram et al., 2008a). LreA and LreB can interact with the red-light sensor FphA which in turn can bind to the velvet protein VeA (Bayram \& Braus, 2012).

In summary, modification of the SAGA complex by SumO displays an interesting possibility to regulate Aspergillus gene repression and to link the SumO network to development. 


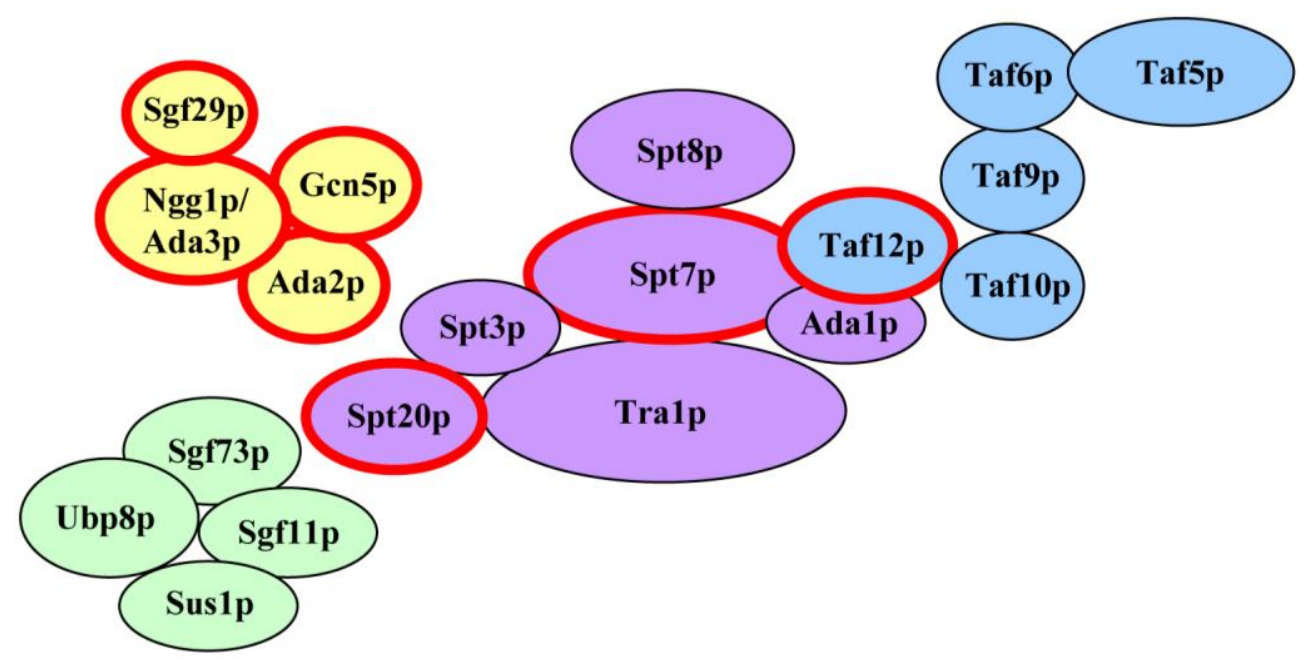

B

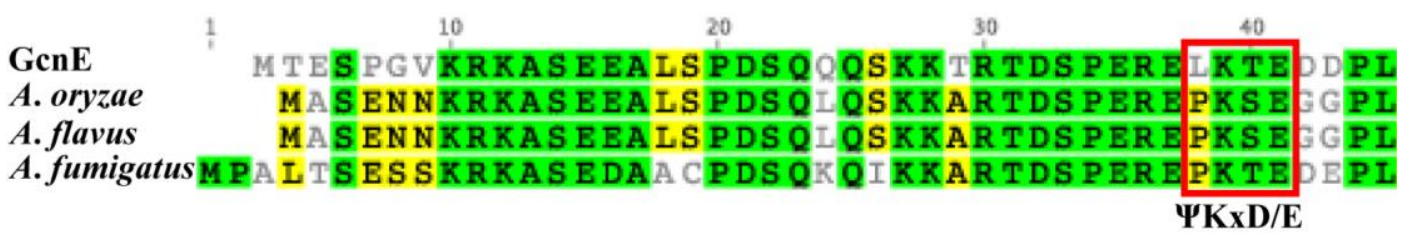

Figure 30: Subunit organization of the yeast SAGA complex.

A Model of the subunit organization of the SAGA complex from S. cerevisiae. The histone acetyltransferase module is shown in yellow, the DUB module in green, the SPT module in purple and the TAF module in blue. Aspergillus homologs of the subunits surrounded by red rings were copurified with TAP::SumO. Modified after (Lee et al., 2011a, Samara \& Wolberger, 2011). B Alignment of the N-terminus of A. nidulans GcnE with putative homologs of A. oryzae (AO090009000327), A. flavus (AFL2G_10350) and A.fumigatus (Afu4g12650). Red box marks the sumoylation motif consisting of four amino acids, $\Psi \mathrm{KxD} / \mathrm{E}$ (with $\Psi$ representing a hydrophobic amino acid and $\mathrm{x}$ any amino acid).

\subsubsection{The SumO network and the COMPASS complex}

Despite the subunits of the SAGA complex, another important regulator of histone modification is linked to the SumO network which is the COMPASS histone methyltransferase complex. The COMPASS complex consists of eight subunits (Set1p, Bre2p, Swd1p, Spp1p, Swd2p, Swd3p, Sdc1p and Shg1p) and is highly conserved: in mammals, seven of the eight yeast subunits have a described homolog. The only exception is Shg1p but loss of Shg1p did not alter the methylation activity of the complex. The detailed subunit organization of the complex is still elusive, 
although crystal structures of domains and core subunits and interaction maps are available (Takahashi et al., 2011). In A.nidulans, all eight yeast subunits are conserved and six of them were co-purified with TAP-tagged SetA.

The core subunit Set1 of the COMPASS complex has been suggested to be sumoylated in yeast (Wohlschlegel et al., 2004). Also another protein of the Set family, human PR-Set7, functions in transcriptional repression and is modified by Sumo1. Cells depleted in the E2 conjugation enzyme Ubc9 revealed a derepression of PR-Set7-regulated genes (Spektor et al., 2011). The COMPASS complex subunit SetA in A. nidulans has seven putative sumoylation motifs and the subunit CclA was co-purified with SumO in our experiments, which makes the COMPASS complex a putative member of the SumO network. The function of the COMPASS complex is connected to gene silencing as well as transcriptional activation (Bernstein et al., 2002, Briggs et al., 2001). The A. nidulans COMPASS and in particular the catalytic subunit SetA were found to be important for sexual development and coordinated secondary metabolism in the fungus. This is consistent with recent findings that the COMPASS complex plays an important role in the initiation of meiotic recombination which is part of the sexual reproduction (Acquaviva et al., 2013). The absence of Set1, accompanied by lower levels of methylation of histone 3 at lysine 4 (H3K4), reduced the initiation of DNA double strand breaks, an important process for meiotic recombination (Borde et al., 2009, Sollier et al., 2004). The induction of DNA double strand breaks heavily depends on the COMPASS subunit Spp1 (Acquaviva et al., 2013).

SetA is additionally required for colony growth and the production of asexual spores. COMPASS provides spatial information for the fungal hyphae to properly position the initials of conidiophores, as in a SetA deficient strain many hyphal tips are terminally differentiated into conidiophores (Figure 31). An intact COMPASS complex allows conidiophore formation from foot cells, located within the filament. This allows the final tip to continue growing and to extend the fungal mycelium which is important to cover surfaces and to access nutrients. It is possible that this phenotype is a result of a defect in hyphal branching and polarity maintenance, as in the deletion strain hyphae repeatedly initiated branching in the long hyphae that ended in conidiophores. These data are consistent with the finding that COMPASS and COMPASS-like complexes are conserved developmental regulators in other organisms, such as A.thaliana (Jiang et al., 2011), Drosophila melanogaster 
(Hallson et al., 2012) or C. elegans (Simonet et al., 2007). The COMPASS complex is also linked to DNA repair pathways, such as the $S$. pombe Set1 homolog (Kanoh et al., 2003, Wood et al., 2005). In A. nidulans the response to DNA damage repair is also important for the regulation of development and is part of the SumO network (see chapter 4.2.1.2.). Additionally, a crosstalk of different histone modification is required for proper regulation of gene expression. Methylation activity of the COMPASS complex depends on ubiquitination of lysine 123 of histone 2 by the Rad6p/Bre1p complex (Eissenberg \& Shilatifard, 2010). This ubiquitin mark can be removed by the SAGA complex subunit Ubp8p (Wood et al., 2005) which makes the COMPASS complex an interface to other putative components of the SumO network in Aspergillus nidulans, namely the SAGA complex and the ubiquitination pathway.

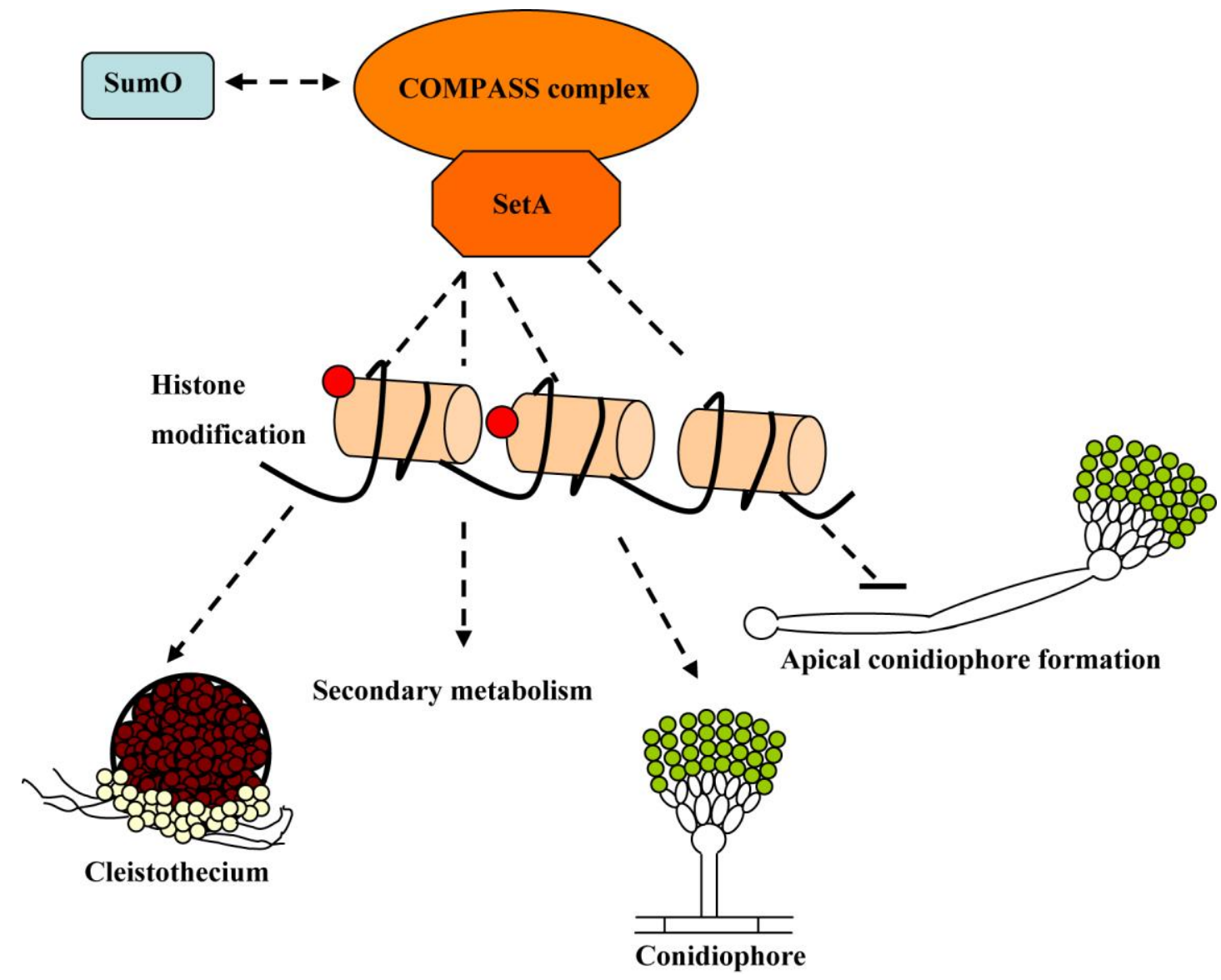

Figure 31: SetA and Aspergillus nidulans development.

The model displays the involvement of SetA in fungal development. SetA is part of the COMPASS complex which acts as histone modifying complex in yeast. In A. nidulans, it is involved in the regulation of sexual reproduction, secondary metabolism and in proper conidiophore development, as it inhibits apical conidiophore formation. The COMPASS complex possibly becomes modified by SumO. 
It is possible that SumO directly modifies one or several subunits of the COMPASS complex. Sumoylation of mammalian PR-Set7 resulted in repression of PR-Set7-regulated genes. Sumoylation of the protein might be the signal for recruitment of corepressors or important for the interaction with DNA. Also the catalytic activity of PR-Set7 might change upon sumoylation (Spektor et al., 2011). Sumo modification of subunits of the COMPASS complex and an impact on the expression of COMPASS-regulated genes needs to be further investigated. The modification might have either a repressive or derepressive function on specific genes as the consequences of COMPASS sumoylation might affect its localization, stability, interactions with other proteins as for example corepressors or with DNA. A temporal or spatial regulated sumoylation might have important effects on the expression of individual genes required for proper fungal development.

\subsection{Outlook}

The neddylation pathway in A. nidulans is essential for cell viability and important for the regulation of the activity of cullin-RING E3 ligases. Several developmentally important Fbox proteins were enriched with NeddH in TAP experiments (von Zeska Kress et al., 2012). Here, it was shown that one of these, Fbox 15, is destabilized in the CSN deneddylase deficient strain, although a subpopulation potentially arrested in a SCF complex, could be biochemically enriched. It would be interesting to identify the substrates for Fbox 15 within the cell to further investigate the reason for the essentiality of the neddylation process as well as the phenotypical consequences of $\operatorname{csn} E$ and $f b o x 15$ deletions. This would be possible by enrichment of Fbox15 interacting proteins through TAP or GFP-trap experiments. However, it might be that the interaction of Fbox 15 with its substrate is too transient or that the tagged protein might not be functional as both, GFP and TAP are quite big tags. Fbox proteins interact with SkpA and additionally with their substrates. Therefore, both, N-terminal as well as C-terminal fusions of the tag might interfere with protein function. Another possibility would be a yeast two hybrid screen with Fbox 15 against an A. nidulans library, although a change of the organism might lead to artificial interactions. Candidates from these approaches have to be individually verified. This could be done by BiFC studies, fusing Fbox 15 to one half of YFP and the putative substrate to the other half of YFP. The observation of a fluorescent signal would than indicate an interaction. 
Additionally, deletion and overexpression strains of putative candidates could be phenotypically analyzed to find the cellular processes they are involved in and stability of the proteins can be monitored in wild type and different deletion strains, as for example $\Delta c s n E$ or $\Delta f b o x 15$, using cycloheximide assays. It is also possible to repeat TAP::NeddH purifications, as the system for MS identification meanwhile is more sensitive compared to the one that was used in previous studies. This would probably allow the identification of other neddylated proteins that might be only present in small amounts in the cell. Furthermore, it would be interesting to investigate which proteins can be co-purified with TAP::NeddH in a strain deficient for the second deneddylase, DenA or in strain, carrying deletions in the genes encoding for both deneddylases, CSN and DenA. This might give further insights in the neddylation and deneddylation process. DcnA functions as auxiliary E3 ligase in the neddylation process, putatively together with RbxA that is also part of the SCF ubiquitin E3 ligase complex. It would be interesting to find proteins that function as E3 ligases for the neddylation of CulB and CulD. Both cullins were also co-purified with TAP-tagged NeddH and are likely to be NeddH-modified (von Zeska Kress et al., 2012). Candidates could be identified with further TAP experiments, although the interactions might be very transient. Deletion strains of corresponding ligases could be constructed and the neddylation levels and the phenotypes can be analyzed. Deletion of $d c n A$ did not result in a phenotype under the investigated conditions. Therefore, other incubation conditions can be tested to find under which the additional ligase function of DcnA is needed.

The sumoylation process is not essential for fungal viability but both, SumO attachment and detachment are important for multicellular development in the fungal cell. Here, a sumoylation network that includes transcriptional regulators, such as histone modifiers, enzymes involved in RNA maturation, stress response proteins, as well as members of the ubiquitination and sumoylation processes was identified. However, the experiments could not distinguish between sumoylated and SumOinteracting proteins. Proteins interacting with sumoylated proteins might be enriched too. Therefore, the candidates should be individually verified to confirm the sumoylation or SumO-interaction. This could be done by BiFC experiments. YFP has a sumoylation site and SumO fused to one part of YFP results in a fluorescent signal, if expressed with the second part of YFP alone (data not shown). This suggests YFP becomes sumoylated by the A. nidulans SumO protein. BiFC 
experiments should be carried out using an YFP protein with an amino acid exchange in the sumoylation motif to exclude unspecific signals. An exchange of the putatively sumoylated lysine to arginine results in an YFP that cannot be modified anymore and could be used to confirm sumoylated proteins.

Another approach to verify the sumoylation of proteins is to use coimmunoprecipitation studies. A putative target protein and its interactors can be enriched by a specific antibody (or an antibody against a tag fused to the protein) and the modification can be confirmed with a SumO-antibody. As many tags and corresponding antibodies cause difficulties in A.nidulans, it is possible to perform the co-immunoprecipitation in S.cerevisiae as a number of tags and antibodies are established in yeast. To increase sumoylation, SumO isopeptidase deficient strains can be used. One isopeptidase, UlpA, was characterized in this study but it is possible that also UlpB and the putative homolog of Wss1p, AN0304, have desumoylating function. To test this, the mature SumO protein can be expressed in the $u l p B$ deletion strain. Analyses of the sumoylation pattern by immunoblotting can be used to monitor a putative desumoylation activity of UlpB despite its suggested SumO maturation function. The phenotype of the strain can also be analyzed to investigate whether the defects of the $u l p B$ deletion strain are explained by a reduction of mature SumO in the fungal cell or whether putative additional functions also lead to phenotypical changes.

The two SumO-isopeptidases Ulp1p and Ulp2p in yeast show different localization patterns within the cell ( $\mathrm{Li} \&$ Hochstrasser, 1999, Li \& Hochstrasser, 2000). As nothing is known about the localization of UlpA and UlpB in A. nidulans, one could fuse the proteins with a fluorescent tag, such as GFP to analyze their subcellular localization. Another possibility is to use immunofluorescent approaches but this would require specific antibodies.

AN0304, the homolog of yeast Wss1p, was not characterized in A. nidulans so far and it would be interesting to see the phenotype and sumoylation pattern of a corresponding deletion strain. Wss1p in yeast was shown to cleave mixed Sumoubiquitin and poly-Sumo chains (Mullen et al., 2010). It is not known, if the SumO protein of $A$. nidulans can form chains, as it misses a classical sumoylation consensus motif. It is possible that the formation of chains occurs at an $\mathrm{N}$-terminal lysine residue. To investigate this, the lysine could be mutated and the phenotype and the sumoylation pattern of the strain can be analyzed. 
Deletion of the SumO E3 enzyme SizA did not result in a strong developmental phenotype. However, it is possible that the ligase activity of the protein is important for conditions that were not tested so far, as for example stress conditions. It would be also interesting to investigate other putative E3 ligases and their cellular functions and impacts on the sumoylation pattern. Therefore, deletion strains of the genes coding for the two additional proteins with Siz/PIAS RING finger-like domains, AN4497 and AN10240, could be constructed and investigated.

Defects in sumoylation have a strong impact on both, sexual and asexual development. The SumO network was investigated under vegetative growth conditions at the time point of developmental competence. Further experiments could give inside on how the substrates of sumoylation change upon induction of asexual and sexual development. It is possible that during development certain substrates are only modified for a strictly regulated timeframe and can be modified by dynamic sumoylation and desumoylation processes. These proteins would be interesting candidates for further research on the connection of sumoylation and fungal development. To find sumoylated or SumO-interacting proteins, the strain expressing TAP::SumO can be used and TAP experiments can be performed with protein extracts from asexual or sexual culture conditions.

In this study, the COMPASS complex was identified as an interface of the sumoylation network and histone modification. The core subunit, SetA, fulfills important functions at the beginning of sexual and asexual development. The COMPASS complex is a histone methyltransferase that is involved in the regulation of gene expression (Eissenberg \& Shilatifard, 2010). As it is not known which genes are regulated by the COMPASS complex, the direct connection to fungal development is still elusive. Whole genome expression arrays could reveal transcripts that are differentially regulated in the setA deletion strain, as for example developmental regulators. A possible impact of sumoylation on the complex function could also be further investigated. In our screen, the subunit CclA was co-purified but in other studies also the modification of Set domain proteins has been suggested (Spektor et al., 2011, Wohlschlegel et al., 2004). Additionally, other proteins that interact with the COMPASS subunit SetA could be identified using the TAP::SetA strain. The data, presented in this work, was derived from identifications with old mass spectrometry equipment. Experiments using a more sensitive device could lead to the identification of additional interaction partners. 


\section{Literature}

Acquaviva, L., L. Szekvolgyi, B. Dichtl, B. S. Dichtl, C. de La Roche Saint Andre, A. Nicolas \& V. Geli, (2013) The COMPASS subunit Spp1 links histone methylation to initiation of meiotic recombination. Science. 339: 215-218.

Adams, T. H., M. T. Boylan \& W. E. Timberlake, (1988) brlA is necessary and sufficient to direct conidiophore development in Aspergillus nidulans. Cell. 54: 353-362.

Adams, T. H., J. K. Wieser \& J. H. Yu, (1998) Asexual sporulation in Aspergillus nidulans. Microbiol Mol Biol Rev. 62: 35-54.

Agarwal, S. \& G. S. Roeder, (2000) Zip3 provides a link between recombination enzymes and synaptonemal complex proteins. Cell. 102: 245-255.

Alseth, I., L. Eide, M. Pirovano, T. Rognes, E. Seeberg \& M. Bjoras, (1999) The Saccharomyces cerevisiae homologues of endonuclease III from Escherichia coli, Ntg1 and Ntg2, are both required for efficient repair of spontaneous and induced oxidative DNA damage in yeast. Mol Cell Biol. 19: 3779-3787.

Arnaud, M. B., G. C. Cerqueira, D. O. Inglis, M. S. Skrzypek, J. Binkley, M. C. Chibucos, J. Crabtree, C. Howarth, J. Orvis, P. Shah, F. Wymore, G. Binkley, S. R. Miyasato, M. Simison, G. Sherlock \& J. R. Wortman, (2012) The Aspergillus Genome Database (AspGD): recent developments in comprehensive multispecies curation, comparative genomics and community resources. Nucleic Acids Res. 40: D653-659.

Arnold, K., L. Bordoli, J. Kopp \& T. Schwede, (2006) The SWISS-MODEL workspace: a web-based environment for protein structure homology modelling. Bioinformatics. 22: 195-201.

Austin, B., R. M. Hall \& B. M. Tyler, (1990) Optimized vectors and selection for transformation of Neurospora crassa and Aspergillus nidulans to bleomycin and phleomycin resistance. Gene. 93: 157-162.

Axelrod, D. E., M. Gealt \& M. Pastushok, (1973) Gene control of developmental competence in Aspergillus nidulans. Dev Biol. 34: 9-15.

Ayaydin, F. \& M. Dasso, (2004) Distinct in vivo dynamics of vertebrate SUMO paralogues. Mol Biol Cell. 15: 5208-5218.

Baba, D., N. Maita, J. G. Jee, Y. Uchimura, H. Saitoh, K. Sugasawa, F. Hanaoka, H. Tochio, H. Hiroaki \& M. Shirakawa, (2005) Crystal structure of thymine DNA glycosylase conjugated to SUMO-1. Nature. 435: 979-982.

Baker, S. E. \& W. J. Bennett, (2007) An Overview of the Genus Aspergillus. In: The Aspergilli. CRC Press, pp. 3-13.

Baud, V. \& E. Derudder, (2011) Control of NF-kappaB activity by proteolysis. Curr Top Microbiol Immunol. 349: 97-114.

Bayer, P., A. Arndt, S. Metzger, R. Mahajan, F. Melchior, R. Jaenicke \& J. Becker, (1998) Structure determination of the small ubiquitin-related modifier SUMO-1. J Mol Biol. 280: 275-286.

Bayram, O., O. S. Bayram, Y. L. Ahmed, J. Maruyama, O. Valerius, S. O. Rizzoli, R. Ficner, S. Irniger \& G. H. Braus, (2012) The Aspergillus nidulans MAPK module AnSte11-Ste50-Ste7-Fus3 controls development and secondary metabolism. PLoS Genet. 8: e1002816.

Bayram, O., C. Biesemann, S. Krappmann, P. Galland \& G. H. Braus, (2008a) More than a repair enzyme: Aspergillus nidulans photolyase-like CryA is a regulator of sexual development. Mol Biol Cell. 19: 3254-3262. 
Bayram, O. \& G. H. Braus, (2012) Coordination of secondary metabolism and development in fungi: the velvet family of regulatory proteins. FEMS Microbiol Rev. 36: 1-24.

Bayram, O., S. Krappmann, M. Ni, J. W. Bok, K. Helmstaedt, O. Valerius, S. BrausStromeyer, N. J. Kwon, N. P. Keller, J. H. Yu \& G. H. Braus, (2008b) VelB/VeA/LaeA complex coordinates light signal with fungal development and secondary metabolism. Science. 320: 1504-1506.

Bayram, O., F. Sari, G. H. Braus \& S. Irniger, (2009) The protein kinase ImeB is required for light-mediated inhibition of sexual development and for mycotoxin production in Aspergillus nidulans. Mol Microbiol. 71: 1278-1295.

Bennet, J. W. \& L. L. Lasure, (1991) Growth media. In: More Gene Manipulation in Fungi. J. W. Bennet \& L. L. Lasure (eds). San Diego: Academic Press Inc., pp. 441-457.

Bergen, L. G. \& N. R. Morris, (1983) Kinetics of the nuclear division cycle of Aspergillus nidulans. J Bacteriol. 156: 155-160.

Bermingham-McDonogh, O., E. B. Gralla \& J. S. Valentine, (1988) The copper, zinc-superoxide dismutase gene of Saccharomyces cerevisiae: cloning, sequencing, and biological activity. Proc Natl Acad Sci U S A. 85: 4789-4793.

Bernier-Villamor, V., D. A. Sampson, M. J. Matunis \& C. D. Lima, (2002) Structural basis for E2-mediated SUMO conjugation revealed by a complex between ubiquitin-conjugating enzyme Ubc9 and RanGAP1. Cell. 108: 345-356.

Bernstein, B. E., E. L. Humphrey, R. L. Erlich, R. Schneider, P. Bouman, J. S. Liu, T. Kouzarides \& S. L. Schreiber, (2002) Methylation of histone H3 Lys 4 in coding regions of active genes. Proceedings of the National Academy of Sciences of the United States of America 99: 8695-8700.

Bertani, G., (1951) Studies on lysogenesis. I. The mode of phage liberation by lysogenic Escherichia coli. J Bacteriol. 62: 293-300.

Bhaumik, S. R., (2011) Distinct regulatory mechanisms of eukaryotic transcriptional activation by SAGA and TFIID. Biochim Biophys Acta. 1809: 97-108.

Blumenstein, A., K. Vienken, R. Tasler, J. Purschwitz, D. Veith, N. FrankenbergDinkel \& R. Fischer, (2005) The Aspergillus nidulans phytochrome FphA represses sexual development in red light. Curr Biol. 15: 1833-1838.

Bok, J. W., Y. M. Chiang, E. Szewczyk, Y. Reyes-Dominguez, A. D. Davidson, J. F. Sanchez, H. C. Lo, K. Watanabe, J. Strauss, B. R. Oakley, C. C. Wang \& N. P. Keller, (2009) Chromatin-level regulation of biosynthetic gene clusters. Nat Chem Biol. 5: 462-464.

Bok, J. W. \& N. P. Keller, (2004) LaeA, a regulator of secondary metabolism in Aspergillus spp. Eukaryot Cell. 3: 527-535.

Borde, V., N. Robine, W. Lin, S. Bonfils, V. Geli \& A. Nicolas, (2009) Histone H3 lysine 4 trimethylation marks meiotic recombination initiation sites. EMBO J. 28: 99-111.

Bordoli, L., F. Kiefer, K. Arnold, P. Benkert, J. Battey \& T. Schwede, (2009) Protein structure homology modeling using SWISS-MODEL workspace. Nat Protoc. 4: 1-13.

Bornstein, G., D. Ganoth \& A. Hershko, (2006) Regulation of neddylation and deneddylation of cullin1 in SCFSkp2 ubiquitin ligase by F-box protein and substrate. Proc Natl Acad Sci U S A. 103: 11515-11520.

Bosu, D. R., H. Feng, K. Min, Y. Kim, M. R. Wallenfang \& E. T. Kipreos, (2010) $C$. elegans CAND-1 regulates cullin neddylation, cell proliferation and morphogenesis in specific tissues. Dev Biol. 346: 113-126. 
Bradford, M. M., (1976) A rapid and sensitive method for the quantitation of microgram quantities of protein utilizing the principle of protein-dye binding. Anal Biochem. 72: 248-254.

Braus, G. H., S. Irniger \& O. Bayram, (2010) Fungal development and the COP9 signalosome. Curr Opin Microbiol. 13: 672-676.

Braus, G. H., S. Krappmann \& S. E. Eckert, (2002) Sexual development in ascomycetes: fruit body formation of Aspergillus nidulans. In: Molecular biology of fungal development. H. D. Osiewacz (ed). New York, Basel: Marcel Decker, Inc., pp. 215-244.

Briggs, S. D., M. Bryk, B. D. Strahl, W. L. Cheung, J. K. Davie, S. Y. Dent, F. Winston \& C. D. Allis, (2001) Histone H3 lysine 4 methylation is mediated by Set1 and required for cell growth and rDNA silencing in Saccharomyces cerevisiae. Genes Dev. 15: 3286-3295.

Bruner, S. D., H. M. Nash, W. S. Lane \& G. L. Verdine, (1998) Repair of oxidatively damaged guanine in Saccharomyces cerevisiae by an alternative pathway. Curr Biol. 8: 393-403.

Buchsbaum, S., B. Bercovich \& A. Ciechanover, (2012) FAT10 is a proteasomal degradation signal that is itself regulated by ubiquitination. Mol Biol Cell. 23: 225-232.

Busch, S., S. E. Eckert, S. Krappmann \& G. H. Braus, (2003) The COP9 signalosome is an essential regulator of development in the filamentous fungus Aspergillus nidulans. Mol Microbiol. 49: 717-730.

Busch, S., E. U. Schwier, K. Nahlik, O. Bayram, K. Helmstaedt, O. W. Draht, S. Krappmann, O. Valerius, W. N. Lipscomb \& G. H. Braus, (2007) An eightsubunit COP9 signalosome with an intact JAMM motif is required for fungal fruit body formation. Proc Natl Acad Sci U S A. 104: 8089-8094.

Bylebyl, G. R., I. Belichenko \& E. S. Johnson, (2003) The SUMO isopeptidase Ulp2 prevents accumulation of SUMO chains in yeast. J Biol Chem. 278: 4411344120.

Chae, K., D. Han \& K. Han, (2007) Sexual Development in Aspergillus nidulans. In: The Aspergilli. CRC Press, pp. 279-299.

Champe, S. P., D. L. Nagle \& L. N. Yager, (1994) Sexual sporulation. Prog Ind Microbiol. 29: 429-454.

Cheng, C. H., Y. H. Lo, S. S. Liang, S. C. Ti, F. M. Lin, C. H. Yeh, H. Y. Huang \& T. F. Wang, (2006) SUMO modifications control assembly of synaptonemal complex and polycomplex in meiosis of Saccharomyces cerevisiae. Genes Dev. 20: 2067-2081.

Cherry, J. M., E. L. Hong, C. Amundsen, R. Balakrishnan, G. Binkley, E. T. Chan, K. R. Christie, M. C. Costanzo, S. S. Dwight, S. R. Engel, D. G. Fisk, J. E. Hirschman, B. C. Hitz, K. Karra, C. J. Krieger, S. R. Miyasato, R. S. Nash, J. Park, M. S. Skrzypek, M. Simison, S. Weng \& E. D. Wong, (2012) Saccharomyces Genome Database: the genomics resource of budding yeast. Nucleic Acids Res. 40: D700-705.

Christmann, M., (2011) Deneddylation and fungal development-Regulation of Nedd8 protein modification by DenA and the COP9 signalosome. Göttingen: Institute of microbiology and genetics, Georg-August University Göttingen.

Christmann, M., T. Schmaler, C. Gordon, X. Huang, O. Bayram, J. Schinke, S. Stumpf, W. Dubiel \& G. H. Braus, (2013) Control of multicellular development by the physically interacting deneddylases DEN1/DenA and COP9 Signalosome. PLoS Genet. 9: e1003275. 
Chuang, H. W., W. Zhang \& W. M. Gray, (2004) Arabidopsis ETA2, an apparent ortholog of the human cullin-interacting protein CAND1, is required for auxin responses mediated by the SCF(TIR1) ubiquitin ligase. Plant Cell. 16: 1883-1897.

Ciechanover, A., H. Heller, S. Elias, A. L. Haas \& A. Hershko, (1980) ATPdependent conjugation of reticulocyte proteins with the polypeptide required for protein degradation. Proc Natl Acad Sci U S A. 77: 1365-1368.

Clutterbuck, A. J., (1969) A mutational analysis of conidial development in Aspergillus nidulans. Genetics. 63: 317-327.

Colabardini, A. C., A. C. Humanes, P. F. Gouvea, M. Savoldi, M. H. Goldman, M. R. Kress, O. Bayram, J. V. Oliveira, M. D. Gomes, G. H. Braus \& G. H. Goldman, (2012) Molecular characterization of the Aspergillus nidulans fbxA encoding an F-box protein involved in xylanase induction. Fungal Genet Biol. 49: $130-140$.

Cope, G. A. \& R. J. Deshaies, (2006) Targeted silencing of Jab1/Csn5 in human cells downregulates SCF activity through reduction of F-box protein levels. BMC Biochem. 7: 1.

Cope, G. A., G. S. Suh, L. Aravind, S. E. Schwarz, S. L. Zipursky, E. V. Koonin \& R. J. Deshaies, (2002) Role of predicted metalloprotease motif of Jab1/Csn5 in cleavage of Nedd8 from Cul1. Science. 298: 608-611.

Denison, C., A. D. Rudner, S. A. Gerber, C. E. Bakalarski, D. Moazed \& S. P. Gygi, (2005) A proteomic strategy for gaining insights into protein sumoylation in yeast. Mol Cell Proteomics. 4: 246-254.

Dharmasiri, S., N. Dharmasiri, H. Hellmann \& M. Estelle, (2003) The RUB/Nedd8 conjugation pathway is required for early development in Arabidopsis. EMBO J. 22: 1762-1770.

Dorval, V. \& P. E. Fraser, (2007) SUMO on the road to neurodegeneration. Biochim Biophys Acta. 1773: 694-706.

Dou, H., C. Huang, T. Van Nguyen, L. S. Lu \& E. T. Yeh, (2011) SUMOylation and de-SUMOylation in response to DNA damage. FEBS Lett. 585: 2891-2896.

$\mathrm{Du}, \mathrm{H} . \mathrm{N}$., (2012) Transcription, DNA damage and beyond: the roles of histone ubiquitination and deubiquitination. Curr Protein Pept Sci. 13: 447-466.

Dubiel, W., (2009) Resolving the CSN and CAND1 paradoxes. Mol Cell. 35: 547549.

Duda, D. M., L. A. Borg, D. C. Scott, H. W. Hunt, M. Hammel \& B. A. Schulman, (2008) Structural insights into NEDD8 activation of cullin-RING ligases: conformational control of conjugation. Cell. 134: 995-1006.

Duda, D. M., D. C. Scott, M. F. Calabrese, E. S. Zimmerman, N. Zheng \& B. A. Schulman, (2011) Structural regulation of cullin-RING ubiquitin ligase complexes. Curr Opin Struct Biol. 21: 257-264.

Dye, B. T. \& B. A. Schulman, (2007) Structural mechanisms underlying posttranslational modification by ubiquitin-like proteins. Annu Rev Biophys Biomol Struct. 36: 131-150.

Dyer, P. S. \& C. M. O'Gorman, (2012) Sexual development and cryptic sexuality in fungi: insights from Aspergillus species. FEMS Microbiol Rev. 36: 165-192.

Eckert, S. E., E. Kubler, B. Hoffmann \& G. H. Braus, (2000) The tryptophan synthase-encoding $\operatorname{trp} B$ gene of Aspergillus nidulans is regulated by the cross-pathway control system. Mol Gen Genet. 263: 867-876.

Eissenberg, J. C. \& A. Shilatifard, (2010) Histone H3 lysine 4 (H3K4) methylation in development and differentiation. Dev Biol. 339: 240-249. 
Enchev, R. I., A. Schreiber, F. Beuron \& E. P. Morris, (2010) Structural insights into the COP9 signalosome and its common architecture with the $26 \mathrm{~S}$ proteasome lid and eIF3. Structure. 18: 518-527.

Enchev, R. I., D. C. Scott, P. C. da Fonseca, A. Schreiber, J. K. Monda, B. A. Schulman, M. Peter \& E. P. Morris, (2012) Structural basis for a reciprocal regulation between SCF and CSN. Cell Rep. 2: 616-627.

Etxebeste, O., A. Garzia, E. A. Espeso \& U. Ugalde, (2010) Aspergillus nidulans asexual development: making the most of cellular modules. Trends Microbiol. 18: 569-576.

Fei, E., N. Jia, M. Yan, Z. Ying, Q. Sun, H. Wang, T. Zhang, X. Ma, H. Ding, X. Yao, Y. Shi \& G. Wang, (2006) SUMO-1 modification increases human SOD1 stability and aggregation. Biochem Biophys Res Commun. 347: 406412.

Ferreira, H. C., B. Luke, H. Schober, V. Kalck, J. Lingner \& S. M. Gasser, (2011) The PIAS homologue Siz2 regulates perinuclear telomere position and telomerase activity in budding yeast. Nat Cell Biol. 13: 867-874.

Flick, K. \& P. Kaiser, (2012) Protein degradation and the stress response. Semin Cell Dev Biol. 23: 515-522.

Frankland-Searby, S. \& S. R. Bhaumik, (2012) The 26S proteasome complex: an attractive target for cancer therapy. Biochim Biophys Acta. 1825: 64-76.

Freilich, S., E. Oron, Y. Kapp, Y. Nevo-Caspi, S. Orgad, D. Segal \& D. A. Chamovitz, (1999) The COP9 signalosome is essential for development of Drosophila melanogaster. Curr Biol. 9: 1187-1190.

Gagne, J. M., B. P. Downes, S. H. Shiu, A. M. Durski \& R. D. Vierstra, (2002) The F-box subunit of the SCF E3 complex is encoded by a diverse superfamily of genes in Arabidopsis. Proc Natl Acad Sci U S A. 99: 11519-11524.

Galagan, J. E., S. E. Calvo, C. Cuomo, L. J. Ma, J. R. Wortman, S. Batzoglou, S. I. Lee, M. Basturkmen, C. C. Spevak, J. Clutterbuck, V. Kapitonov, J. Jurka, C. Scazzocchio, M. Farman, J. Butler, S. Purcell, S. Harris, G. H. Braus, O. Draht, S. Busch, C. D'Enfert, C. Bouchier, G. H. Goldman, D. Bell-Pedersen, S. Griffiths-Jones, J. H. Doonan, J. Yu, K. Vienken, A. Pain, M. Freitag, E. U. Selker, D. B. Archer, M. A. Penalva, B. R. Oakley, M. Momany, T. Tanaka, T. Kumagai, K. Asai, M. Machida, W. C. Nierman, D. W. Denning, M. Caddick, M. Hynes, M. Paoletti, R. Fischer, B. Miller, P. Dyer, M. S. Sachs, S. A. Osmani \& B. W. Birren, (2005) Sequencing of Aspergillus nidulans and comparative analysis with A. fumigatus and A. oryzae. Nature. 438: 11051115.

Gan-Erdene, T., K. Nagamalleswari, L. Yin, K. Wu, Z. Q. Pan \& K. D. Wilkinson, (2003) Identification and characterization of DEN1, a deneddylase of the ULP family. J Biol Chem. 278: 28892-28900.

Garcia, I., M. Mathieu, I. Nikolaev, B. Felenbok \& C. Scazzocchio, (2008) Roles of the Aspergillus nidulans homologues of Tup1 and Ssn6 in chromatin structure and cell viability. FEMS Microbiol Lett. 289: 146-154.

Garza, R. \& L. Pillus, (2013) STUbLs in chromatin and genome stability. Biopolymers. 99: 146-154.

Geiss-Friedlander, R. \& F. Melchior, (2007) Concepts in sumoylation: a decade on. Nat Rev Mol Cell Biol. 8: 947-956.

Georgianna, D. R. \& G. A. Payne, (2009) Genetic regulation of aflatoxin biosynthesis: from gene to genome. Fungal Genet Biol. 46: 113-125. 
Gerke, J., O. Bayram \& G. H. Braus, (2012) Fungal S-adenosylmethionine synthetase and the control of development and secondary metabolism in Aspergillus nidulans. Fungal Genet Biol. 49: 443-454.

Giles, S. S., A. A. Soukup, C. Lauer, M. Shaaban, A. Lin, B. R. Oakley, C. C. Wang \& N. P. Keller, (2011) Cryptic Aspergillus nidulans antimicrobials. Appl Environ Microbiol. 77: 3669-3675.

Gill, G., (2005) Something about SUMO inhibits transcription. Curr Opin Genet Dev. 15: $536-541$.

Gnad, F., J. Gunawardena \& M. Mann, (2011) PHOSIDA 2011: the posttranslational modification database. Nucleic Acids Res. 39: D253-260.

Gnad, F., S. Ren, J. Cox, J. V. Olsen, B. Macek, M. Oroshi \& M. Mann, (2007) PHOSIDA (phosphorylation site database): management, structural and evolutionary investigation, and prediction of phosphosites. Genome Biol. 8: R250.

Goldenberg, S. J., T. C. Cascio, S. D. Shumway, K. C. Garbutt, J. Liu, Y. Xiong \& N. Zheng, (2004) Structure of the Cand1-Cul1-Roc1 complex reveals regulatory mechanisms for the assembly of the multisubunit cullin-dependent ubiquitin ligases. Cell. 119: 517-528.

Goldstein, G., M. Scheid, U. Hammerling, D. H. Schlesinger, H. D. Niall \& E. A. Boyse, (1975) Isolation of a polypeptide that has lymphocyte-differentiating properties and is probably represented universally in living cells. Proc Natl Acad Sci U S A. 72: 11-15.

Grimaldi, B., P. Coiro, P. Filetici, E. Berge, J. R. Dobosy, M. Freitag, E. U. Selker \& P. Ballario, (2006) The Neurospora crassa White Collar-1 dependent blue light response requires acetylation of histone H3 lysine 14 by NGF-1. Mol Biol Cell. 17: 4576-4583.

Guo, D., M. Li, Y. Zhang, P. Yang, S. Eckenrode, D. Hopkins, W. Zheng, S. Purohit, R. H. Podolsky, A. Muir, J. Wang, Z. Dong, T. Brusko, M. Atkinson, P. Pozzilli, A. Zeidler, L. J. Raffel, C. O. Jacob, Y. Park, M. Serrano-Rios, M. T. Larrad, Z. Zhang, H. J. Garchon, J. F. Bach, J. I. Rotter, J. X. She \& C. Y. Wang, (2004) A functional variant of SUMO4, a new I kappa B alpha modifier, is associated with type 1 diabetes. Nat Genet. 36: 837-841.

Hagiwara, D., Y. Asano, J. Marui, K. Furukawa, K. Kanamaru, M. Kato, K. Abe, T. Kobayashi, T. Yamashino \& T. Mizuno, (2007) The SskA and SrrA response regulators are implicated in oxidative stress responses of hyphae and asexual spores in the phosphorelay signaling network of Aspergillus nidulans. Biosci Biotechnol Biochem. 71: 1003-1014.

Hallson, G., R. E. Hollebakken, T. Li, M. Syrzycka, I. Kim, S. Cotsworth, K. A. Fitzpatrick, D. A. Sinclair \& B. M. Honda, (2012) dSet1 is the main H3K4 di- and tri-methyltransferase throughout Drosophila development. Genetics. 190: $91-100$.

Han, K. H., K. Y. Han, J. H. Yu, K. S. Chae, K. Y. Jahng \& D. M. Han, (2001) The $n s d D$ gene encodes a putative GATA-type transcription factor necessary for sexual development of Aspergillus nidulans. Mol Microbiol. 41: 299-309.

Han, S. \& T. H. Adams, (2001) Complex control of the developmental regulatory locus brlA in Aspergillus nidulans. Mol Genet Genomics. 266: 260-270.

Hannich, J. T., A. Lewis, M. B. Kroetz, S. J. Li, H. Heide, A. Emili \& M. Hochstrasser, (2005) Defining the SUMO-modified proteome by multiple approaches in Saccharomyces cerevisiae. J Biol Chem. 280: 4102-4110. 
Hardeland, U., R. Steinacher, J. Jiricny \& P. Schar, (2002) Modification of the human thymine-DNA glycosylase by ubiquitin-like proteins facilitates enzymatic turnover. EMBO J. 21: 1456-1464.

Harris, S. D., (1999) Morphogenesis is coordinated with nuclear division in germinating Aspergillus nidulans conidiospores. Microbiology. 145 ( Pt 10): 2747-2756.

Harris, S. D., (2007) Hyphal Morphogenesis in Aspergillus nidulans. In: The Aspergilli. CRC Press, pp. 211-222.

Harting, R., Ö. Bayram, K. Laubinger, O. Valerius \& G. H. Braus, (2013) Interplay of the fungal sumoylation network for control of multicellular development. Mol Microbiol. DOI: 10.11111/mmi.12421.

Hedayati, M. T., A. C. Pasqualotto, P. A. Warn, P. Bowyer \& D. W. Denning, (2007) Aspergillus flavus: human pathogen, allergen and mycotoxin producer. Microbiology. 153: 1677-1692.

Helmstaedt, K., K. Laubinger, K. Vosskuhl, O. Bayram, S. Busch, M. Hoppert, O. Valerius, S. Seiler \& G. H. Braus, (2008) The nuclear migration protein NUDF/LIS1 forms a complex with NUDC and BNFA at spindle pole bodies. Eukaryot Cell. 7: 1041-1052.

Helmstaedt, K., E. U. Schwier, M. Christmann, K. Nahlik, M. Westermann, R. Harting, S. Grond, S. Busch \& G. H. Braus, (2011) Recruitment of the inhibitor Cand1 to the cullin substrate adaptor site mediates interaction to the neddylation site. Mol Biol Cell. 22: 153-164.

Henriques, R., I. C. Jang \& N. H. Chua, (2009) Regulated proteolysis in light-related signaling pathways. Curr Opin Plant Biol. 12: 49-56.

Hershko, A., A. Ciechanover, H. Heller, A. L. Haas \& I. A. Rose, (1980) Proposed role of ATP in protein breakdown: conjugation of protein with multiple chains of the polypeptide of ATP-dependent proteolysis. Proc Natl Acad Sci U S A. 77: 1783-1786.

Hicks, J., R. A. Lockington, J. Strauss, D. Dieringer, C. P. Kubicek, J. Kelly \& N. Keller, (2001) RcoA has pleiotropic effects on Aspergillus nidulans cellular development. Mol Microbiol. 39: 1482-1493.

Hietakangas, V., J. Anckar, H. A. Blomster, M. Fujimoto, J. J. Palvimo, A. Nakai \& L. Sistonen, (2006) PDSM, a motif for phosphorylation-dependent SUMO modification. Proc Natl Acad Sci U S A. 103: 45-50.

Hochstrasser, M., (1996) Ubiquitin-dependent protein degradation. Annu Rev Genet. 30: $405-439$.

Hochstrasser, M., (2009) Origin and function of ubiquitin-like proteins. Nature. 458 : 422-429.

Hofmann, K. \& P. Bucher, (1998) The PCI domain: a common theme in three multiprotein complexes. Trends Biochem Sci 23: 204-205.

Hu, C. D., Y. Chinenov \& T. K. Kerppola, (2002) Visualization of interactions among bZIP and Rel family proteins in living cells using bimolecular fluorescence complementation. Mol Cell. 9: 789-798.

Hunter, S., P. Jones, A. Mitchell, R. Apweiler, T. K. Attwood, A. Bateman, T. Bernard, D. Binns, P. Bork, S. Burge, E. de Castro, P. Coggill, M. Corbett, U. Das, L. Daugherty, L. Duquenne, R. D. Finn, M. Fraser, J. Gough, D. Haft, N. Hulo, D. Kahn, E. Kelly, I. Letunic, D. Lonsdale, R. Lopez, M. Madera, J. Maslen, C. McAnulla, J. McDowall, C. McMenamin, H. Mi, P. MutowoMuellenet, N. Mulder, D. Natale, C. Orengo, S. Pesseat, M. Punta, A. F. Quinn, C. Rivoire, A. Sangrador-Vegas, J. D. Selengut, C. J. Sigrist, M. Scheremetjew, J. Tate, M. Thimmajanarthanan, P. D. Thomas, C. H. Wu, C. 
Yeats \& S. Y. Yong, (2012) InterPro in 2011: new developments in the family and domain prediction database. Nucleic Acids Res. 40: D306-312.

Hwang, J. W., K. W. Min, T. A. Tamura \& J. B. Yoon, (2003) TIP120A associates with unneddylated cullin 1 and regulates its neddylation. FEBS Lett. 541: 102-108.

Inoue, H., H. Nojima \& H. Okayama, (1990) High efficiency transformation of Escherichia coli with plasmids. Gene. 96: 23-28.

Jackson, S. P. \& D. Durocher, (2013) Regulation of DNA damage responses by Ubiquitin and SUMO. Mol Cell. 49: 795-807.

Jedd, G. \& L. Pieuchot, (2012) Multiple modes for gatekeeping at fungal cell-to-cell channels. Mol Microbiol. 86: 1291-1294.

Jiang, D., N. C. Kong, X. Gu, Z. Li \& Y. He, (2011) Arabidopsis COMPASS-like complexes mediate histone $\mathrm{H} 3$ lysine-4 trimethylation to control floral transition and plant development. PLoS Genet. 7: e1001330.

Jin, J., T. Cardozo, R. C. Lovering, S. J. Elledge, M. Pagano \& J. W. Harper, (2004) Systematic analysis and nomenclature of mammalian F-box proteins. Genes Dev. 18: 2573-2580.

Johnson, E. S. \& G. Blobel, (1997) Ubc9p is the conjugating enzyme for the ubiquitin-like protein Smt3p. J Biol Chem. 272: 26799-26802.

Johnson, E. S. \& A. A. Gupta, (2001) An E3-like factor that promotes SUMO conjugation to the yeast septins. Cell. 106: 735-744.

Johnson, E. S., I. Schwienhorst, R. J. Dohmen \& G. Blobel, (1997) The ubiquitinlike protein $\mathrm{Smt} 3 \mathrm{p}$ is activated for conjugation to other proteins by an Aos1p/Uba2p heterodimer. EMBO J. 16: 5509-5519.

Johnson, P. R. \& M. Hochstrasser, (1997) SUMO-1: Ubiquitin gains weight. Trends Cell Biol. 7: 408-413.

Jones, D. \& E. P. Candido, (2000) The NED-8 conjugating system in Caenorhabditis elegans is required for embryogenesis and terminal differentiation of the hypodermis. Dev Biol. 226: 152-165.

Kadosh, D. \& K. Struhl, (1998) Targeted recruitment of the Sin3-Rpd3 histone deacetylase complex generates a highly localized domain of repressed chromatin in vivo. Mol Cell Biol. 18: 5121-5127.

Käfer, E., (1977) Meiotic and mitotic recombination in Aspergillus and its chromosomal aberrations. Adv Genet. 19: 33-131.

Kanoh, J., S. Francesconi, A. Collura, V. Schramke, F. Ishikawa, G. Baldacci \& V. Geli, (2003) The fission yeast spSet1p is a histone H3-K4 methyltransferase that functions in telomere maintenance and DNA repair in an ATM kinase Rad3-dependent pathway. J Mol Biol. 326: 1081-1094.

Kapelari, B., D. Bech-Otschir, R. Hegerl, R. Schade, R. Dumdey \& W. Dubiel, (2000) Electron microscopy and subunit-subunit interaction studies reveal a first architecture of COP9 signalosome. J Mol Biol. 300: 1169-1178.

Kato, N., W. Brooks \& A. M. Calvo, (2003) The expression of sterigmatocystin and penicillin genes in Aspergillus nidulans is controlled by veA, a gene required for sexual development. Eukaryot Cell. 2: 1178-1186.

Kerscher, O., (2007) SUMO junction-what's your function? New insights through SUMO-interacting motifs. EMBO Rep. 8: 550-555.

Kerscher, O., R. Felberbaum \& M. Hochstrasser, (2006) Modification of proteins by ubiquitin and ubiquitin-like proteins. Annu Rev Cell Dev Biol. 22: 159-180.

Kiefer, F., K. Arnold, M. Kunzli, L. Bordoli \& T. Schwede, (2009) The SWISSMODEL Repository and associated resources. Nucleic Acids Res. 37: D387392. 
Kim, H., K. Han, K. Kim, D. Han, K. Jahng \& K. Chae, (2002) The veA gene activates sexual development in Aspergillus nidulans. Fungal Genet Biol. 37: 72-80.

Knuf, C. \& J. Nielsen, (2012) Aspergilli: systems biology and industrial applications. Biotechnol J. 7: 1147-1155.

Krappmann, S., N. Jung, B. Medic, S. Busch, R. A. Prade \& G. H. Braus, (2006) The Aspergillus nidulans F-box protein GrrA links SCF activity to meiosis. Mol Microbiol. 61: 76-88.

Krugel, H., G. Fiedler, C. Smith \& S. Baumberg, (1993) Sequence and transcriptional analysis of the nourseothricin acetyltransferase-encoding gene nat1 from Streptomyces noursei. Gene. 127: 127-131.

Kubodera, T., N. Yamashita \& A. Nishimura, (2000) Pyrithiamine resistance gene (ptrA) of Aspergillus oryzae: cloning, characterization and application as a dominant selectable marker for transformation. Biosci Biotechnol Biochem. 64: 1416-1421.

Kumar, S., Y. Tomooka \& M. Noda, (1992) Identification of a set of genes with developmentally down-regulated expression in the mouse brain. Biochem Biophys Res Commun. 185: 1155-1161.

Kumar, S., Y. Yoshida \& M. Noda, (1993) Cloning of a cDNA which encodes a novel ubiquitin-like protein. Biochem Biophys Res Commun. 195: 393-399.

Kundu, M. \& C. B. Thompson, (2008) Autophagy: basic principles and relevance to disease. Annu Rev Pathol. 3: 427-455.

Kurepa, J., J. M. Walker, J. Smalle, M. M. Gosink, S. J. Davis, T. L. Durham, D. Y. Sung \& R. D. Vierstra, (2003) The small ubiquitin-like modifier (SUMO) protein modification system in Arabidopsis. Accumulation of SUMO1 and -2 conjugates is increased by stress. J Biol Chem. 278: 6862-6872.

Kurz, T., Y. C. Chou, A. R. Willems, N. Meyer-Schaller, M. L. Hecht, M. Tyers, M. Peter \& F. Sicheri, (2008) Dcn1 functions as a scaffold-type E3 ligase for cullin neddylation. Mol Cell. 29: 23-35.

Kurz, T., N. Ozlu, F. Rudolf, S. M. O'Rourke, B. Luke, K. Hofmann, A. A. Hyman, B. Bowerman \& M. Peter, (2005) The conserved protein DCN-1/Dcn1p is required for cullin neddylation in $C$. elegans and $S$. cerevisiae. Nature. 435: 1257-1261.

Lara-Ortiz, T., H. Riveros-Rosas \& J. Aguirre, (2003) Reactive oxygen species generated by microbial NADPH oxidase NoxA regulate sexual development in Aspergillus nidulans. Mol Microbiol. 50: 1241-1255.

Larsen, C. N., J. S. Price \& K. D. Wilkinson, (1996) Substrate binding and catalysis by ubiquitin C-terminal hydrolases: identification of two active site residues. Biochemistry. 35: 6735-6744.

Laubinger, K., (2008) Analysis of neuronal diseases in the model organism Aspergillus nidulans. Göttingen: Institute of microbiology and genetics, Georg-August University Göttingen.

Lee, B. Y., S. Y. Han, H. G. Choi, J. H. Kim, K. H. Han \& D. M. Han, (2005) Screening of growth- or development-related genes by using genomic library with inducible promoter in Aspergillus nidulans. J Microbiol. 43: 523-528.

Lee, K. K., M. E. Sardiu, S. K. Swanson, J. M. Gilmore, M. Torok, P. A. Grant, L. Florens, J. L. Workman \& M. P. Washburn, (2011a) Combinatorial depletion analysis to assemble the network architecture of the SAGA and ADA chromatin remodeling complexes. Molecular systems biology 7: 503.

Lee, M. H., R. Zhao, L. Phan \& S. C. Yeung, (2011b) Roles of COP9 signalosome in cancer. Cell Cycle. 10: 3057-3066. 
Lee, S. B. \& J. W. Taylor, (1990) Isolation of DNA from fungal mycelia and single spores. In: PCR protocols: A guide to methods and applications. N. Innis, D. Gelfand, J. Sninsky \& T. White (eds). New York: Academic Press, pp. 282287.

Leidel, S., P. G. Pedrioli, T. Bucher, R. Brost, M. Costanzo, A. Schmidt, R. Aebersold, C. Boone, K. Hofmann \& M. Peter, (2009) Ubiquitin-related modifier Urm1 acts as a sulphur carrier in thiolation of eukaryotic transfer RNA. Nature. 458: 228-232.

Lenschow, D. J., (2010) Antiviral properties of ISG15. Viruses. 2: 2154-2168.

Li, S. J. \& M. Hochstrasser, (1999) A new protease required for cell-cycle progression in yeast. Nature. 398: 246-251.

Li, S. J. \& M. Hochstrasser, (2000) The yeast ULP2 (SMT4) gene encodes a novel protease specific for the ubiquitin-like Smt3 protein. Mol Cell Biol. 20: 23672377.

Liakopoulos, D., G. Doenges, K. Matuschewski \& S. Jentsch, (1998) A novel protein modification pathway related to the ubiquitin system. EMBO J. 17: 22082214.

Lima, J. F., I. Malavazi, M. R. von Zeska Kress Fagundes, M. Savoldi, M. H. Goldman, E. Schwier, G. H. Braus \& G. H. Goldman, (2005) The $c s n D / c s n E$ signalosome genes are involved in the Aspergillus nidulans DNA damage response. Genetics. 171: 1003-1015.

Liu, J., M. Furukawa, T. Matsumoto \& Y. Xiong, (2002) NEDD8 modification of CUL1 dissociates p120(CAND1), an inhibitor of CUL1-SKP1 binding and SCF ligases. Mol Cell. 10: 1511-1518.

Lloyd, A. T. \& P. M. Sharp, (1991) Codon usage in Aspergillus nidulans. Mol Gen Genet. 230: 288-294.

Lo, S. C. \& M. Hannink, (2006) CAND1-mediated substrate adaptor recycling is required for efficient repression of Nrf2 by Keap1. Mol Cell Biol. 26: 12351244.

Loeb, K. R. \& A. L. Haas, (1992) The interferon-inducible 15-kDa ubiquitin homolog conjugates to intracellular proteins. J Biol Chem. 267: 7806-7813.

Longo, V. D., E. B. Gralla \& J. S. Valentine, (1996) Superoxide dismutase activity is essential for stationary phase survival in Saccharomyces cerevisiae.

Mitochondrial production of toxic oxygen species in vivo. J Biol Chem. 271: 12275-12280.

Lyst, M. J. \& I. Stancheva, (2007) A role for SUMO modification in transcriptional repression and activation. Biochem Soc Trans. 35: 1389-1392.

Ma, T., Y. Chen, F. Zhang, C. Y. Yang, S. Wang \& X. Yu, (2013) RNF111dependent neddylation activates DNA damage-induced ubiquitination. $\mathrm{Mol}$ Cell. 49: 897-907.

Mabey Gilsenan, J., J. Cooley \& P. Bowyer, (2012) CADRE: the Central Aspergillus Data REpository 2012. Nucleic Acids Res. 40: D660-666.

Malavazi, I., M. Savoldi, S. M. Di Mauro, C. F. Menck, S. D. Harris, M. H. Goldman \& G. H. Goldman, (2006) Transcriptome analysis of Aspergillus nidulans exposed to camptothecin-induced DNA damage. Eukaryot Cell. 5: 1688-1704.

Marshall, M. A. \& W. E. Timberlake, (1991) Aspergillus nidulans wetA activates spore-specific gene expression. Mol Cell Biol. 11: 55-62.

Matic, I., J. Schimmel, I. A. Hendriks, M. A. van Santen, F. van de Rijke, H. van Dam, F. Gnad, M. Mann \& A. C. Vertegaal, (2010) Site-specific identification of SUMO-2 targets in cells reveals an inverted SUMOylation motif and a hydrophobic cluster SUMOylation motif. Mol Cell. 39: 641-652. 
Matic, I., M. van Hagen, J. Schimmel, B. Macek, S. C. Ogg, M. H. Tatham, R. T. Hay, A. I. Lamond, M. Mann \& A. C. Vertegaal, (2008) In vivo identification of human small ubiquitin-like modifier polymerization sites by high accuracy mass spectrometry and an in vitro to in vivo strategy. Mol Cell Proteomics. 7: 132-144.

Matunis, M. J., E. Coutavas \& G. Blobel, (1996) A novel ubiquitin-like modification modulates the partitioning of the Ran-GTPase-activating protein RanGAP1 between the cytosol and the nuclear pore complex. J Cell Biol. 135: 14571470 .

Mayorga, M. E. \& W. E. Timberlake, (1990) Isolation and molecular characterization of the Aspergillus nidulans wA gene. Genetics. 126: 73-79.

McCluskey, K., A. Wiest \& M. Plamann, (2010) The Fungal Genetics Stock Center: a repository for 50 years of fungal genetics research. J Biosci. 35: 119-126.

McCormick, A., J. Loeffler \& F. Ebel, (2010) Aspergillus fumigatus: contours of an opportunistic human pathogen. Cell Microbiol. 12: 1535-1543.

McGrath, J. P., S. Jentsch \& A. Varshavsky, (1991) UBA 1: an essential yeast gene encoding ubiquitin-activating enzyme. EMBO J. 10: 227-236.

Melchior, F., M. Schergaut \& A. Pichler, (2003) SUMO: ligases, isopeptidases and nuclear pores. Trends Biochem Sci. 28: 612-618.

Merlet, J., J. Burger, J. E. Gomes \& L. Pintard, (2009) Regulation of cullin-RING E3 ubiquitin-ligases by neddylation and dimerization. Cell Mol Life Sci. 66: 1924-1938.

Morimoto, M., T. Nishida, Y. Nagayama \& H. Yasuda, (2003) Nedd8-modification of Cul1 is promoted by Roc1 as a Nedd8-E3 ligase and regulates its stability. Biochem Biophys Res Commun. 301: 392-398.

Morris, J. R., (2010) More modifiers move on DNA damage. Cancer Res. 70: 38613863.

Morris, J. R., C. Boutell, M. Keppler, R. Densham, D. Weekes, A. Alamshah, L. Butler, Y. Galanty, L. Pangon, T. Kiuchi, T. Ng \& E. Solomon, (2009) The SUMO modification pathway is involved in the BRCA1 response to genotoxic stress. Nature. 462: 886-890.

Mukhopadhyay, D. \& M. Dasso, (2007) Modification in reverse: the SUMO proteases. Trends Biochem Sci. 32: 286-295.

Mullen, J. R., C. F. Chen \& S. J. Brill, (2010) Wss1 is a SUMO-dependent isopeptidase that interacts genetically with the Slx5-Slx 8 SUMO-targeted ubiquitin ligase. Mol Cell Biol. 30: 3737-3748.

Mummery-Widmer, J. L., M. Yamazaki, T. Stoeger, M. Novatchkova, S. Bhalerao, D. Chen, G. Dietzl, B. J. Dickson \& J. A. Knoblich, (2009) Genome-wide analysis of Notch signalling in Drosophila by transgenic RNAi. Nature. 458 : 987-992.

Nacerddine, K., F. Lehembre, M. Bhaumik, J. Artus, M. Cohen-Tannoudji, C. Babinet, P. P. Pandolfi \& A. Dejean, (2005) The SUMO pathway is essential for nuclear integrity and chromosome segregation in mice. Dev Cell. 9: 769779.

Nahlik, K., M. Dumkow, O. Bayram, K. Helmstaedt, S. Busch, O. Valerius, J. Gerke, M. Hoppert, E. Schwier, L. Opitz, M. Westermann, S. Grond, K. Feussner, C. Goebel, A. Kaever, P. Meinicke, I. Feussner \& G. H. Braus, (2010) The COP9 signalosome mediates transcriptional and metabolic response to hormones, oxidative stress protection and cell wall rearrangement during fungal development. Mol Microbiol. 78: 964-979. 
Nakayama, K. I. \& K. Nakayama, (2005) Regulation of the cell cycle by SCF-type ubiquitin ligases. Semin Cell Dev Biol. 16: 323-333.

Natorff, R., M. Balinska \& A. Paszewski, (1993) At least four regulatory genes control sulphur metabolite repression in Aspergillus nidulans. Mol Gen Genet. 238: $185-192$.

Nayak, T., E. Szewczyk, C. E. Oakley, A. Osmani, L. Ukil, S. L. Murray, M. J. Hynes, S. A. Osmani \& B. R. Oakley, (2006) A versatile and efficient genetargeting system for Aspergillus nidulans. Genetics. 172: 1557-1566.

Ni, M. \& J. H. Yu, (2007) A novel regulator couples sporogenesis and trehalose biogenesis in Aspergillus nidulans. PLoS One. 2: e970.

O'Brien, K. M., R. Dirmeier, M. Engle \& R. O. Poyton, (2004) Mitochondrial protein oxidation in yeast mutants lacking manganese-(MnSOD) or copper- and zinccontaining superoxide dismutase (CuZnSOD): evidence that MnSOD and $\mathrm{CuZnSOD}$ have both unique and overlapping functions in protecting mitochondrial proteins from oxidative damage. J Biol Chem. 279: 5181751827.

O'Hara, E. B. \& W. E. Timberlake, (1989) Molecular characterization of the Aspergillus nidulans yA locus. Genetics. 121: 249-254.

Oberegger, H., I. Zadra, M. Schoeser \& H. Haas, (2000) Iron starvation leads to increased expression of $\mathrm{Cu} / \mathrm{Zn}$-superoxide dismutase in Aspergillus. FEBS Lett. 485: 113-116.

Osmani, A. H., B. R. Oakley \& S. A. Osmani, (2006) Identification and analysis of essential Aspergillus nidulans genes using the heterokaryon rescue technique. Nat Protoc. 1: 2517-2526.

Palvimo, J. J., (2007) PIAS proteins as regulators of small ubiquitin-related modifier (SUMO) modifications and transcription. Biochem Soc Trans. 35: 1405-1408.

Panse, V. G., U. Hardeland, T. Werner, B. Kuster \& E. Hurt, (2004) A proteomewide approach identifies sumoylated substrate proteins in yeast. J Biol Chem. 279: 41346-41351.

Park, H. S. \& J. H. Yu, (2012) Genetic control of asexual sporulation in filamentous fungi. Curr Opin Microbiol. 15: 669-677.

Petroski, M. D. \& R. J. Deshaies, (2005) Function and regulation of cullin-RING ubiquitin ligases. Nat Rev Mol Cell Biol. 6: 9-20.

Pfaffl, M. W., G. W. Horgan \& L. Dempfle, (2002) Relative expression software tool (REST) for group-wise comparison and statistical analysis of relative expression results in real-time PCR. Nucleic Acids Res. 30: e36.

Pichler, A., A. Gast, J. S. Seeler, A. Dejean \& F. Melchior, (2002) The nucleoporin RanBP2 has SUMO1 E3 ligase activity. Cell. 108: 109-120.

Pichler, A., P. Knipscheer, H. Saitoh, T. K. Sixma \& F. Melchior, (2004) The RanBP2 SUMO E3 ligase is neither HECT- nor RING-type. Nat Struct Mol Biol. 11: 984-991.

Pick, E., K. Hofmann \& M. H. Glickman, (2009) PCI complexes: Beyond the proteasome, CSN, and eIF3 Troika. Mol Cell. 35: 260-264.

Pickart, C. M. \& M. J. Eddins, (2004) Ubiquitin: structures, functions, mechanisms. Biochim Biophys Acta. 1695: 55-72.

Pickett, M., D. I. Gwynne, F. P. Buxton, R. Elliott, R. W. Davies, R. A. Lockington, C. Scazzocchio \& H. M. Sealy-Lewis, (1987) Cloning and characterization of the aldA gene of Aspergillus nidulans. Gene. 51: 217-226.

Pickles, S. \& C. Vande Velde, (2012) Misfolded SOD1 and ALS: zeroing in on mitochondria. Amyotroph Lateral Scler. 13: 333-340. 
Pitt, C. W., E. Moreau, P. A. Lunness \& J. H. Doonan, (2004) The pot1+ homologue in Aspergillus nidulans is required for ordering mitotic events. J Cell Sci. 117: 199-209.

Pontecorvo, G., J. A. Roper, L. M. Hemmons, K. D. Macdonald \& A. W. Bufton, (1953) The genetics of Aspergillus nidulans. Adv Genet. 5: 141-238.

Prade, R. A. \& W. E. Timberlake, (1993) The Aspergillus nidulans brlA regulatory locus consists of overlapping transcription units that are individually required for conidiophore development. EMBO J. 12: 2439-2447.

Praefcke, G. J., K. Hofmann \& R. J. Dohmen, (2012) SUMO playing tag with ubiquitin. Trends Biochem Sci. 37: 23-31.

Purschwitz, J., S. Muller \& R. Fischer, (2009) Mapping the interaction sites of Aspergillus nidulans phytochrome FphA with the global regulator VeA and the White Collar protein LreB. Mol Genet Genomics. 281: 35-42.

Purschwitz, J., S. Muller, C. Kastner, M. Schoser, H. Haas, E. A. Espeso, A. Atoui, A. M. Calvo \& R. Fischer, (2008) Functional and physical interaction of blue- and red-light sensors in Aspergillus nidulans. Curr Biol. 18: 255-259.

Quevillon, E., V. Silventoinen, S. Pillai, N. Harte, N. Mulder, R. Apweiler \& R. Lopez, (2005) InterProScan: protein domains identifier. Nucleic Acids Res 33: W116-120.

Rabut, G. \& M. Peter, (2008) Function and regulation of protein neddylation. 'Protein modifications: beyond the usual suspects' review series. EMBO Rep. 9: 969-976.

Rajendra, R., D. Malegaonkar, P. Pungaliya, H. Marshall, Z. Rasheed, J. Brownell, L. F. Liu, S. Lutzker, A. Saleem \& E. H. Rubin, (2004) Topors functions as an E3 ubiquitin ligase with specific E2 enzymes and ubiquitinates p53. J Biol Chem. 279: 36440-36444.

Reyes-Dominguez, Y., F. Narendja, H. Berger, A. Gallmetzer, R. Fernandez-Martin, I. Garcia, C. Scazzocchio \& J. Strauss, (2008) Nucleosome positioning and histone $\mathrm{H} 3$ acetylation are independent processes in the Aspergillus nidulans prnD-prnB bidirectional promoter. Eukaryot Cell. 7: 656-663.

Riederer, B. M., G. Leuba, A. Vernay \& I. M. Riederer, (2011) The role of the ubiquitin proteasome system in Alzheimer's disease. Exp Biol Med (Maywood). 236: 268-276.

Rodriguez, M. S., C. Dargemont \& R. T. Hay, (2001) SUMO-1 conjugation in vivo requires both a consensus modification motif and nuclear targeting. $J$ Biol Chem. 276: 12654-12659.

Roguev, A., D. Schaft, A. Shevchenko, W. W. Pijnappel, M. Wilm, R. Aasland \& A. F. Stewart, (2001) The Saccharomyces cerevisiae Set1 complex includes an Ash2 homologue and methylates histone 3 lysine 4. EMBO J. 20: 7137-7148.

Rytinki, M. M., S. Kaikkonen, P. Pehkonen, T. Jaaskelainen \& J. J. Palvimo, (2009) PIAS proteins: pleiotropic interactors associated with SUMO. Cell Mol Life Sci. 66: 3029-3041.

Saha, A. \& R. J. Deshaies, (2008) Multimodal activation of the ubiquitin ligase SCF by Nedd8 conjugation. Mol Cell. 32: 21-31.

Samara, N. L. \& C. Wolberger, (2011) A new chapter in the transcription SAGA. Curr Opin Struct Biol. 21: 767-774.

Sambrook, J., E. F. Fritsch \& T. Maniatis, (1989) Molecular Cloning: A Laboratory Manual. Cold Spring Harbor Laboratory Press, New York.

Saracco, S. A., M. J. Miller, J. Kurepa \& R. D. Vierstra, (2007) Genetic analysis of SUMOylation in Arabidopsis: conjugation of SUMO1 and SUMO2 to nuclear proteins is essential. Plant Physiol. 145: 119-134. 
Sarikas, A., T. Hartmann \& Z. Q. Pan, (2011) The cullin protein family. Genome Biol. 12: 220.

Sarikaya Bayram, O., O. Bayram, O. Valerius, H. S. Park, S. Irniger, J. Gerke, M. Ni, K. H. Han, J. H. Yu \& G. H. Braus, (2010) LaeA control of velvet family regulatory proteins for light-dependent development and fungal cell-type specificity. PLoS Genet. 6: e1001226.

Scheel, H. \& K. Hofmann, (2005) Prediction of a common structural scaffold for proteasome lid, COP9-signalosome and eIF3 complexes. BMC Bioinformatics. 6: 71.

Schwechheimer, C. \& X. W. Deng, (2001) COP9 signalosome revisited: a novel mediator of protein degradation. Trends Cell Biol. 11: 420-426.

Scott, D. C., J. K. Monda, C. R. Grace, D. M. Duda, R. W. Kriwacki, T. Kurz \& B. A. Schulman, (2010) A dual E3 mechanism for Rub1 ligation to Cdc53. Mol Cell. 39: 784-796.

Semighini, C. P. \& S. D. Harris, (2008) Regulation of apical dominance in Aspergillus nidulans hyphae by reactive oxygen species. Genetics. 179: 19191932.

Seol, J. H., R. M. Feldman, W. Zachariae, A. Shevchenko, C. C. Correll, S. Lyapina, Y. Chi, M. Galova, J. Claypool, S. Sandmeyer, K. Nasmyth \& R. J. Deshaies, (1999) Cdc53/cullin and the essential Hrt1 RING-H2 subunit of SCF define a ubiquitin ligase module that activates the E2 enzyme Cdc34. Genes Dev. 13: 1614-1626.

Sewall, T. C., C. W. Mims \& W. E. Timberlake, (1990) abaA controls phialide differentiation in Aspergillus nidulans. Plant Cell. 2: 731-739.

Sharon, M., H. Mao, E. Boeri Erba, E. Stephens, N. Zheng \& C. V. Robinson, (2009) Symmetrical modularity of the COP9 signalosome complex suggests its multifunctionality. Structure. 17: 31-40.

Shevchenko, A., M. Wilm, O. Vorm \& M. Mann, (1996) Mass spectrometric sequencing of proteins silver-stained polyacrylamide gels. Anal Chem. 68: 850-858.

Shpilka, T., H. Weidberg, S. Pietrokovski \& Z. Elazar, (2011) Atg8: an autophagyrelated ubiquitin-like protein family. Genome biology 12: 226.

Siergiejuk, E., D. C. Scott, B. A. Schulman, K. Hofmann, T. Kurz \& M. Peter, (2009) Cullin neddylation and substrate-adaptors counteract SCF inhibition by the CAND1-like protein Lag2 in Saccharomyces cerevisiae. EMBO J. 28: 38453856.

Silverstein, R. A. \& K. Ekwall, (2005) Sin3: a flexible regulator of global gene expression and genome stability. Curr Genet. 47: 1-17.

Simonet, T., R. Dulermo, S. Schott \& F. Palladino, (2007) Antagonistic functions of SET-2/SET1 and HPL/HP1 proteins in C. elegans development. Dev Biol. 312: $367-383$.

Skilton, A., J. C. Ho, B. Mercer, E. Outwin \& F. Z. Watts, (2009) SUMO chain formation is required for response to replication arrest in S. pombe. PLoS One. 4: e6750.

Skowyra, D., K. L. Craig, M. Tyers, S. J. Elledge \& J. W. Harper, (1997) F-box proteins are receptors that recruit phosphorylated substrates to the SCF ubiquitin-ligase complex. Cell. 91: 209-219.

Smith, R. L. \& A. D. Johnson, (2000) Turning genes off by Ssn6-Tup1: a conserved system of transcriptional repression in eukaryotes. Trends Biochem Sci. 25: 325-330. 
Sollier, J., W. Lin, C. Soustelle, K. Suhre, A. Nicolas, V. Geli \& C. de La Roche Saint-Andre, (2004) Set1 is required for meiotic S-phase onset, double-strand break formation and middle gene expression. EMBO J. 23: 1957-1967.

Southern, E. M., (1975) Detection of specific sequences among DNA fragments separated by gel electrophoresis. Journal of molecular biology 98: 503-517.

Spektor, T. M., L. M. Congdon, C. S. Veerappan \& J. C. Rice, (2011) The UBC9 E2 SUMO conjugating enzyme binds the PR-Set7 histone methyltransferase to facilitate target gene repression. PLoS One. 6: e22785.

Sterner, D. E., D. Nathan, A. Reindle, E. S. Johnson \& S. L. Berger, (2006) Sumoylation of the yeast Gcn5 protein. Biochemistry. 45: 1035-1042.

Stinnett, S. M., E. A. Espeso, L. Cobeno, L. Araujo-Bazan \& A. M. Calvo, (2007) Aspergillus nidulans VeA subcellular localization is dependent on the importin alpha carrier and on light. Mol Microbiol. 63: 242-255.

Stratmann, J. W. \& G. Gusmaroli, (2012) Many jobs for one good cop - the COP9 signalosome guards development and defense. Plant Sci. 185-186: 50-64.

Szewczyk, E., Y. M. Chiang, C. E. Oakley, A. D. Davidson, C. C. Wang \& B. R. Oakley, (2008) Identification and characterization of the asperthecin gene cluster of Aspergillus nidulans. Appl Environ Microbiol. 74: 7607-7612.

Szewczyk, E., T. Nayak, C. E. Oakley, H. Edgerton, Y. Xiong, N. Taheri-Talesh, S. A. Osmani \& B. R. Oakley, (2006) Fusion PCR and gene targeting in Aspergillus nidulans. Nat Protoc. 1: 3111-3120.

Takahashi, Y., T. Kahyo, E. A. Toh, H. Yasuda \& Y. Kikuchi, (2001a) Yeast Ul11/Siz1 is a novel SUMO1/Smt3 ligase for septin components and functions as an adaptor between conjugating enzyme and substrates. $J$ Biol Chem. 276: 48973-48977.

Takahashi, Y., A. Toh-e \& Y. Kikuchi, (2001b) A novel factor required for the SUMO1/Smt3 conjugation of yeast septins. Gene. 275: 223-231.

Takahashi, Y. H., G. H. Westfield, A. N. Oleskie, R. C. Trievel, A. Shilatifard \& G. Skiniotis, (2011) Structural analysis of the core COMPASS family of histone H3K4 methylases from yeast to human. Proceedings of the National Academy of Sciences of the United States of America 108: 20526-20531.

Tan, J. M., E. S. Wong \& K. L. Lim, (2009) Protein misfolding and aggregation in Parkinson's disease. Antioxid Redox Signal. 11: 2119-2134.

Tateishi, K., M. Omata, K. Tanaka \& T. Chiba, (2001) The NEDD8 system is essential for cell cycle progression and morphogenetic pathway in mice. $J$ Cell Biol. 155: 571-579.

Tatham, M. H., E. Jaffray, O. A. Vaughan, J. M. Desterro, C. H. Botting, J. H. Naismith \& R. T. Hay, (2001) Polymeric chains of SUMO-2 and SUMO-3 are conjugated to protein substrates by SAE1/SAE2 and Ubc9. J Biol Chem. 276: 35368-35374.

Timberlake, W. E., (1990) Molecular genetics of Aspergillus development. Annu Rev Genet. 24: 5-36.

Timberlake, W. E. \& A. J. Clutterbuck, (1994) Genetic regulation of conidiation. Prog Ind Microbiol. 29: 383-427.

Todd, R. B., M. J. Hynes \& A. Andrianopoulos, (2006) The Aspergillus nidulans $r c o A$ gene is required for $v e A$-dependent sexual development. Genetics. 174: 1685-1688.

Tomoda, K., N. Yoneda-Kato, A. Fukumoto, S. Yamanaka \& J. Y. Kato, (2004) Multiple functions of Jab1 are required for early embryonic development and growth potential in mice. J Biol Chem. 279: 43013-43018. 
Trujillo, K. M., R. K. Tyler, C. Ye, S. L. Berger \& M. A. Osley, (2011) A genetic and molecular toolbox for analyzing histone ubiquitylation and sumoylation in yeast. Methods. 54: 296-303.

Tsitsigiannis, D. I., R. Zarnowski \& N. P. Keller, (2004) The lipid body protein, PpoA, coordinates sexual and asexual sporulation in Aspergillus nidulans. $J$ Biol Chem. 279: 11344-11353.

Tu, S., E. M. Bulloch, L. Yang, C. Ren, W. C. Huang, P. H. Hsu, C. H. Chen, C. L. Liao, H. M. Yu, W. S. Lo, M. A. Freitas \& M. D. Tsai, (2007) Identification of histone demethylases in Saccharomyces cerevisiae. J Biol Chem. 282: 14262-14271.

Uzunova, K., K. Gottsche, M. Miteva, S. R. Weisshaar, C. Glanemann, M. Schnellhardt, M. Niessen, H. Scheel, K. Hofmann, E. S. Johnson, G. J. Praefcke \& R. J. Dohmen, (2007) Ubiquitin-dependent proteolytic control of SUMO conjugates. J Biol Chem. 282: 34167-34175.

Vallim, M. A., K. Y. Miller \& B. L. Miller, (2000) Aspergillus SteA (sterile12-like) is a homeodomain- $\mathrm{C} 2 / \mathrm{H} 2-\mathrm{Zn}+2$ finger transcription factor required for sexual reproduction. Mol Microbiol. 36: 290-301.

van der Veen, A. G. \& H. L. Ploegh, (2012) Ubiquitin-like proteins. Annu Rev Biochem. 81: 323-357.

Van Dross, R. T., K. V. Rao, E. Eisenberg \& M. M. Sanders, (1997) Cloning and characterization of the Aspergillus nidulans DNA topoisomerase I gene. Gene. 203: 169-174.

Vargas-Perez, I., O. Sanchez, L. Kawasaki, D. Georgellis \& J. Aguirre, (2007) Response regulators SrrA and SskA are central components of a phosphorelay system involved in stress signal transduction and asexual sporulation in Aspergillus nidulans. Eukaryot Cell. 6: 1570-1583.

Vertegaal, A. C., J. S. Andersen, S. C. Ogg, R. T. Hay, M. Mann \& A. I. Lamond, (2006) Distinct and overlapping sets of SUMO-1 and SUMO-2 target proteins revealed by quantitative proteomics. Mol Cell Proteomics. 5: 22982310.

von Zeska Kress, M. R., R. Harting, O. Bayram, M. Christmann, H. Irmer, O. Valerius, J. Schinke, G. H. Goldman \& G. H. Braus, (2012) The COP9 signalosome counteracts the accumulation of cullin SCF ubiquitin E3 RING ligases during fungal development. Mol Microbiol. 83: 1162-1177.

Wang, C. Y. \& J. X. She, (2008) SUMO4 and its role in type 1 diabetes pathogenesis. Diabetes Metab Res Rev. 24: 93-102.

Wang, J., (2011) Cardiac function and disease: emerging role of small ubiquitinrelated modifier. Wiley Interdiscip Rev Syst Biol Med. 3: 446-457.

Wang, Y. \& M. Dasso, (2009) SUMOylation and deSUMOylation at a glance. J Cell Sci. 122: 4249-4252.

Watson, A. D., D. G. Edmondson, J. R. Bone, Y. Mukai, Y. Yu, W. Du, D. J. Stillman \& S. Y. Roth, (2000) Ssn6-Tup1 interacts with class I histone deacetylases required for repression. Genes Dev. 14: 2737-2744.

Weger, S., E. Hammer \& R. Heilbronn, (2005) Topors acts as a SUMO-1 E3 ligase for p53 in vitro and in vivo. FEBS Lett. 579: 5007-5012.

Wei, N. \& X. W. Deng, (1992) COP9: a new genetic locus involved in lightregulated development and gene expression in Arabidopsis. Plant Cell. 4: 1507-1518.

Wei, N. \& X. W. Deng, (1999) Making sense of the COP9 signalosome. A regulatory protein complex conserved from Arabidopsis to human. Trends Genet. 15: 98-103. 
Wei, N. \& X. W. Deng, (2003) The COP9 signalosome. Annu Rev Cell Dev Biol. 19: 261-286.

Wei, N., G. Serino \& X. W. Deng, (2008) The COP9 signalosome: more than a protease. Trends Biochem Sci. 33: 592-600.

Wilkinson, K. A. \& J. M. Henley, (2010) Mechanisms, regulation and consequences of protein SUMOylation. Biochem J. 428: 133-145.

Wilkinson, K. D., (2000) Ubiquitination and deubiquitination: targeting of proteins for degradation by the proteasome. Semin Cell Dev Biol. 11: 141-148.

Wohlschlegel, J. A., E. S. Johnson, S. I. Reed \& J. R. Yates, 3rd, (2004) Global analysis of protein sumoylation in Saccharomyces cerevisiae. J Biol Chem. 279: 45662-45668.

Wolkow, T. D., S. D. Harris \& J. E. Hamer, (1996) Cytokinesis in Aspergillus nidulans is controlled by cell size, nuclear positioning and mitosis. J Cell Sci. 109 ( Pt 8): 2179-2188.

Wong, K. H., R. B. Todd, B. R. Oakley, C. E. Oakley, M. J. Hynes \& M. A. Davis, (2008) Sumoylation in Aspergillus nidulans: sumO inactivation, overexpression and live-cell imaging. Fungal Genet Biol. 45: 728-737.

Wood, A., J. Schneider \& A. Shilatifard, (2005) Cross-talking histones: implications for the regulation of gene expression and DNA repair. Biochem Cell Biol. 83: 460-467.

Wood, V., M. A. Harris, M. D. McDowall, K. Rutherford, B. W. Vaughan, D. M. Staines, M. Aslett, A. Lock, J. Bahler, P. J. Kersey \& S. G. Oliver, (2012) PomBase: a comprehensive online resource for fission yeast. Nucleic Acids Res. 40: D695-699.

Woodcock, D. M., P. J. Crowther, J. Doherty, S. Jefferson, E. DeCruz, M. NoyerWeidner, S. S. Smith, M. Z. Michael \& M. W. Graham, (1989) Quantitative evaluation of Escherichia coli host strains for tolerance to cytosine methylation in plasmid and phage recombinants. Nucleic Acids Res. 17: 3469-3478.

Wu, K., K. Yamoah, G. Dolios, T. Gan-Erdene, P. Tan, A. Chen, C. G. Lee, N. Wei, K. D. Wilkinson, R. Wang \& Z. Q. Pan, (2003) DEN1 is a dual function protease capable of processing the $\mathrm{C}$ terminus of Nedd8 and deconjugating hyper-neddylated CUL1. J Biol Chem. 278: 28882-28891.

Xie, Y., O. Kerscher, M. B. Kroetz, H. F. McConchie, P. Sung \& M. Hochstrasser, (2007) The yeast Hex3.Slx 8 heterodimer is a ubiquitin ligase stimulated by substrate sumoylation. J Biol Chem. 282: 34176-34184.

Yager, L. N., (1992) Early developmental events during asexual and sexual sporulation in Aspergillus nidulans. Biotechnology. 23: 19-41.

Yang, S. H., A. Galanis, J. Witty \& A. D. Sharrocks, (2006) An extended consensus motif enhances the specificity of substrate modification by SUMO. EMBO J. 25: 5083-5093.

Yeh, E. T., (2009) SUMOylation and De-SUMOylation: wrestling with life's processes. J Biol Chem. 284: 8223-8227.

Yoshimoto, H., M. Ohmae \& I. Yamashita, (1992) The Saccharomyces cerevisiae GAM2/SIN3 protein plays a role in both activation and repression of transcription. Mol Gen Genet. 233: 327-330.

Zhao, J., (2007) Sumoylation regulates diverse biological processes. Cell Mol Life Sci. 64: 3017-3033.

Zhao, X. \& G. Blobel, (2005) A SUMO ligase is part of a nuclear multiprotein complex that affects DNA repair and chromosomal organization. Proc Natl Acad Sci U S A. 102: 4777-4782. 
Zheng, N., B. A. Schulman, L. Song, J. J. Miller, P. D. Jeffrey, P. Wang, C. Chu, D. M. Koepp, S. J. Elledge, M. Pagano, R. C. Conaway, J. W. Conaway, J. W. Harper \& N. P. Pavletich, (2002) Structure of the Cul1-Rbx1-Skp1-F boxSkp2 SCF ubiquitin ligase complex. Nature. 416: 703-709.

Zhou, L. \& F. Z. Watts, (2005) Nep1, a Schizosaccharomyces pombe deneddylating enzyme. Biochem J. 389: 307-314.

Zhou, W., J. J. Ryan \& H. Zhou, (2004) Global analyses of sumoylated proteins in Saccharomyces cerevisiae. Induction of protein sumoylation by cellular stresses. J Biol Chem. 279: 32262-32268. 


\section{Abbreviations}

A

$\AA$

Ala

ALS

ampR

Asp

ATP

$\mathrm{BiFC}$

BLAST

bp

C

CAN

CBB

CEB

cDNA

CID

$\mathrm{cm}$

COP

COMPASS

CRL

CSN

C-terminus

D

D

Da

DAPI

DIC

DMF

DNA

DTT

DUB

E alanine (Ala)

Ångström

alanine

amyotrophic lateral sclerosis

ampicillin resistance

Aspartic acid

adenosine triphosphate

bi-molecular fluorescence complementation

basic local alignment search tool

base pair

cysteine (Cys)

Canada

Calmodulin binding buffer

Calmodulin elution buffer

complementary DNA

collision-induced decay

centimeter

constitutive photomorphogenic

complex proteins associated with Set1

cullin-RING ligase

COP9-signalosome

carboxy terminus

Germany

aspartic acid (Asp)

Dalton

4',6-diamidino-2-phenylindole

differential interference contrast

$\mathrm{N}, \mathrm{N}$-dimethylformamide

deoxyribonucleic acid

dithiothreitol

deubiquitinating

glutamic acid (Glu) 


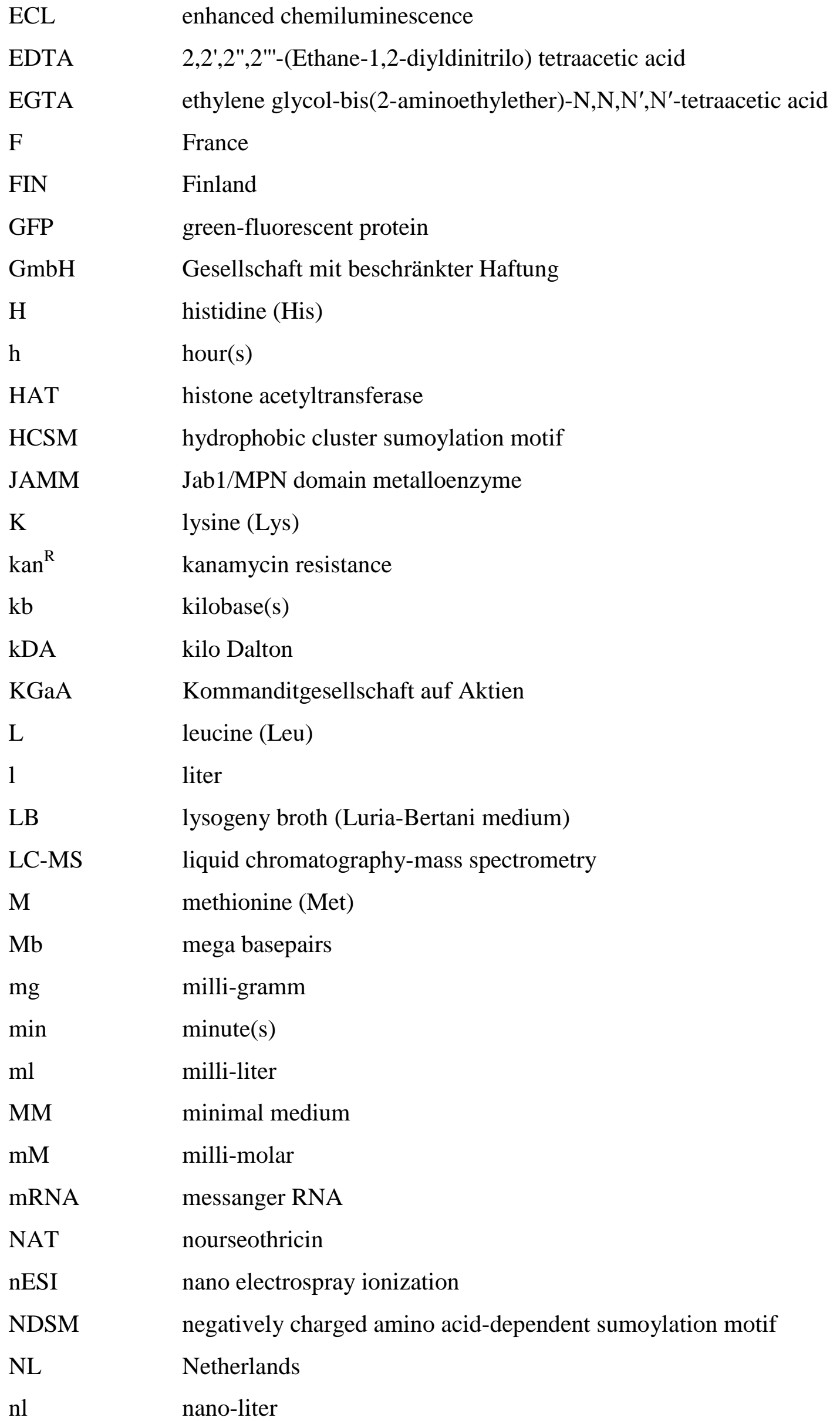




\begin{tabular}{|c|c|}
\hline N-terminus & amino terminus \\
\hline $\mathrm{NZ}$ & New Zealand \\
\hline $\mathrm{P}$ & proline (Pro) \\
\hline PAGE & polyacrylamide gel electrophoresis \\
\hline PCR & polymerase chain reaction \\
\hline PDSM & phosphorylation-dependent sumoylation motif \\
\hline PIAS & protein inhibitor of activated STAT \\
\hline PIM & protease inhibitor mix \\
\hline PINIT & proteasome subunits, Int- 6 , Nip-1, and TRIP-15 \\
\hline PMSF & phenylmethanesulfonyl fluoride \\
\hline PONY & potentiating neddylation \\
\hline ppm & parts per million \\
\hline ptrA & pyridthyamin \\
\hline Q & glutamine (Gln) \\
\hline qRT & qantitative real-time \\
\hline rcf & relative centrifugal force \\
\hline RNA & ribonucleic acid \\
\hline RT & room temperature \\
\hline$S$ & serine (ser) \\
\hline $\mathrm{s}$ & second(s) \\
\hline SAGA & Spt-Ada-Gcn5-Acetyltransferase \\
\hline SAP & scaffold attachment factor-A/B, acinus and PIAS \\
\hline $\mathrm{SCF}$ & Skp1-Cullin-Fbox protein \\
\hline SDS & sodium dodecyl sulfate \\
\hline Senp & Sentrin/Sumo-specific protease \\
\hline SIM & Sumo interaction motif \\
\hline SRUbL & Sumo-regulated ubiquitin ligase \\
\hline ST & sterigmatocystin \\
\hline STAT & signal transducer and activator of transcription \\
\hline STUbL & Sumo-targeted ubiquitin ligases \\
\hline $\mathrm{T}$ & threonine (Thr) \\
\hline $\mathrm{t}$ & time \\
\hline TAP & tandem affinity purification \\
\hline TBP & TATA-binding protein \\
\hline
\end{tabular}




$\begin{array}{ll}\text { TCA } & \text { trichloroacetic acid } \\ \text { TLC } & \text { thin layer chromatography } \\ \text { TEV } & \text { tabacco etch virus } \\ \text { Tris } & \text { 2-Amino-2-hydroxymethyl-propane-1,3-diol } \\ \text { tRNA } & \text { transfer RNA } \\ \text { UBA } & \text { ubiquitin-associated } \\ \text { UBL } & \text { ubiquitin-like } \\ \text { UK } & \text { United kingdom } \\ \text { Ulp } & \text { ubiquitin-like protein-specific protease } \\ \text { USA } & \text { United States of America } \\ \text { UTR } & \text { untranslated region } \\ \text { UV } & \text { ultra-violett } \\ \text { V } & \text { valine (Val) } \\ \text { wt } & \text { wild type } \\ \text { YFP } & \text { yellow fluorescent protein }\end{array}$




\section{Table of figures}

Figure 1: Structures of ubiquitin-like family proteins. 5

$\begin{array}{ll}\text { Figure 2: The ubiquitination reaction. } & 8\end{array}$

Figure 3: Structure of Den1p.

Figure 4: Model of the architecture of the COP9 signalosome. 11

$\begin{array}{ll}\text { Figure 5: Possible consequences of sumoylation for substrate proteins. } & 14\end{array}$

Figure 6: Domain structure of Siz/PIAS proteins. 16

Figure 7: Life cycle of Aspergillus nidulans. 21

Figure 8: Light dependent regulation of development. 24

Figure 9: The Aspergillus nidulans SumO protein in comparison to $\begin{array}{ll}\text { human Sumo1. } & 27\end{array}$

Figure 10: DenA interacts with components of the neddylation and ubiquitination machinery. $\quad 58$

Figure 11: Deletion of $d c n A$ allows normal development in A. nidulans. $\quad 59$

Figure 12: Deletion of $d c n A$ moderately decreases the cellular levels of neddylated cullins. $\quad 60$

Figure 13: Deletion of the CSN deneddylase increases the levels of $\begin{array}{ll}\text { neddylated cullins. } & 61\end{array}$

Figure 14: The CSN stabilizes Fbox15-GFP. 62

Figure 15: Sequence alignment of SumO, Smt3 and human Sumo1-3. 65

Figure 16: The sumO deletion strain shows defects in light control and multicellular development. 66

Figure 17: Deletion of $u l p A$ reduces the amount of asexual spores 68

Figure 18: Deletion of $u l p A$ changes the cellular sumoylation pattern. $\quad 70$

Figure 19: UlpB is a SumO processing enzyme. 71

Figure 20: TAP::SumO recruits potentially sumoylated and SumO-interacting $\begin{array}{ll}\text { proteins. } & 74\end{array}$

Figure 21: Deletions of $a o s A, u b a B, u b c N$ and sizA change the cellular sumoylation pattern.

Figure 22: Deletions of $a o s A, u b a B$ and $u b c N$ affect asexual and sexual $\begin{array}{ll}\text { development. } & 80\end{array}$

Figure 23: Deletion of setA leads to an early block in sexual development. $\quad 84$

Figure 24: The setA deletion strain is impaired in asexual development. 86 
Figure 25: Deletion of setA leads to unusual positioning of the conidiophore. 87

Figure 26: The neddylation pathway in A. nidulans. $\quad 90$

Figure 27: The SumO network of Aspergillus nidulans. 94

Figure 28: Sumo E3 ligases of S. cerevisiae and A. nidulans. 96

Figure 29: Interplay of neddylation, ubiquitination and sumoylation in $\begin{array}{ll}\text { DNA repair. } & 101\end{array}$

Figure 30: Subunit organization of the yeast SAGA complex. 105

Figure 31: SetA and Aspergillus nidulans development. 107 


\section{List of tables}

$\begin{array}{ll}\text { Table 1: Sumoylation motifs. } & 17\end{array}$

Table 2: Human Senps and yeast Ulps. Modified after (Yeh, 2009). 19

Table 3: Plasmids used in this study. 30

Table 4: Primers used in this study.

Table 5: A. nidulans strains used in this study. 37

$\begin{array}{ll}\text { Table 6: Proteins co-purified with TAP-SumO. } & 75\end{array}$

Table 7: Proteins co-purified with SetA-TAP. $\quad 82$ 


\section{Acknowledgements}

I would like to thank Prof Dr. Gerhard H. Braus for supervision and support during my PhD study. I appreciate his time, advice and the chance to participate in a lot of interesting national and international conferences.

I also want to thank Prof Dr. Andrea Polle as member of my thesis committee for her advices and suggestions.

I am very grateful to Dr. Özgür Bayram for his help, ideas and his time for discussions and proof reading of my thesis. Furthermore I would like to thank him for all strains, plasmids and primers he shared with me. I also wish to thank Gabriele Heinrich for her technical assistance and for all the support in every day's lab life. Furthermore, I want to thank Dr. Martin Christmann for being a kind colleague, for all fruitful discussions and advices, for proof reading of my thesis, for solving my computer problems and guiding me through foreign conference cities. I am very thankful for the support of Josua S. Schinke during his practical courses, his diploma thesis and as a colleague. Special thanks for reading my thesis, for following my scientific studies and for always being helpful!

I also want to thank my current and former lab members Dr. Elena Beckmann, Dr. Mirit Kolog-Gulko, Dr. Marcia Regina von Zeska Kress and Dr. Kerstin Helmstaedt for their personal and scientific support and ideas.

I wish to thank Dr. Henriette Irmer for helping me with my RT-PCR experiments and for proof reading of this thesis and Dr. Jennifer Gerke for answering all 'chemical questions' and reading parts of my thesis. I am grateful to Dr. Oliver Valerius, Verena Hofer-Pretz and Kerstin Schmitt for handling all my MS samples. Special thanks go to all the students, who worked with me during my thesis and who contributed with their experiments: Till Heller, Anne Bartels and Ute Wünn. I also wish to thank all other members of the department, supporting me and my work. Thanks for discussions, suggestions, friendly words and chocolate!

I am also grateful to my friends and family for their help and support. Especially, I want to thank Dr. Andrea Thiemann for reading my thesis and providing an insight into a physicist view of biology. My thanks also go to my brother Klaas and Benedikt Obermeier for their great computer assistance. Special thanks go to my boyfriend Christian, who always encouraged me and supported my work. 


\title{
Curriculum vitae
}

\author{
Personal details: \\ Rebekka Harting \\ born on May $19^{\text {th }}, 1983$ \\ in Göttingen, Germany
}

\section{Education}

1989 - 1995 Primary education at the Grundschule Dransfeld and the Schule am Hohen Hagen in Dransfeld (Orientierungsstufe).

1995 - 2002 Secondary education at the Otto-Hahn-Gymnasium in Göttingen (Abitur: university entrance diploma).

\section{Scientific background}

$2002-2007$ Study of biology at the Georg-August Universität in Göttingen.

2007

Diploma thesis ,Sumoylierung verhindert den Wachstumsdefekt verursacht durch Überexpression von A30P- $\alpha$-Synuklein in Aspergillus nidulans" in the Department of Molecular Microbiology and Genetics at the Georg-August Universität in Göttingen (Prof. Braus).

since 2008 Scientific assistant and PhD student in the lab of Prof. Gerhard Braus at the Georg-August Universität Göttingen. 